\title{
INFLAMMATION IN THE CNS: ADVANCING THE FIELD USING INTRAVITAL IMAGING
}

EDITED BY: Saparna Pai, Michael J. Hickey, and Wolfgang Weninger PUBLISHED IN: Frontiers in Immunology

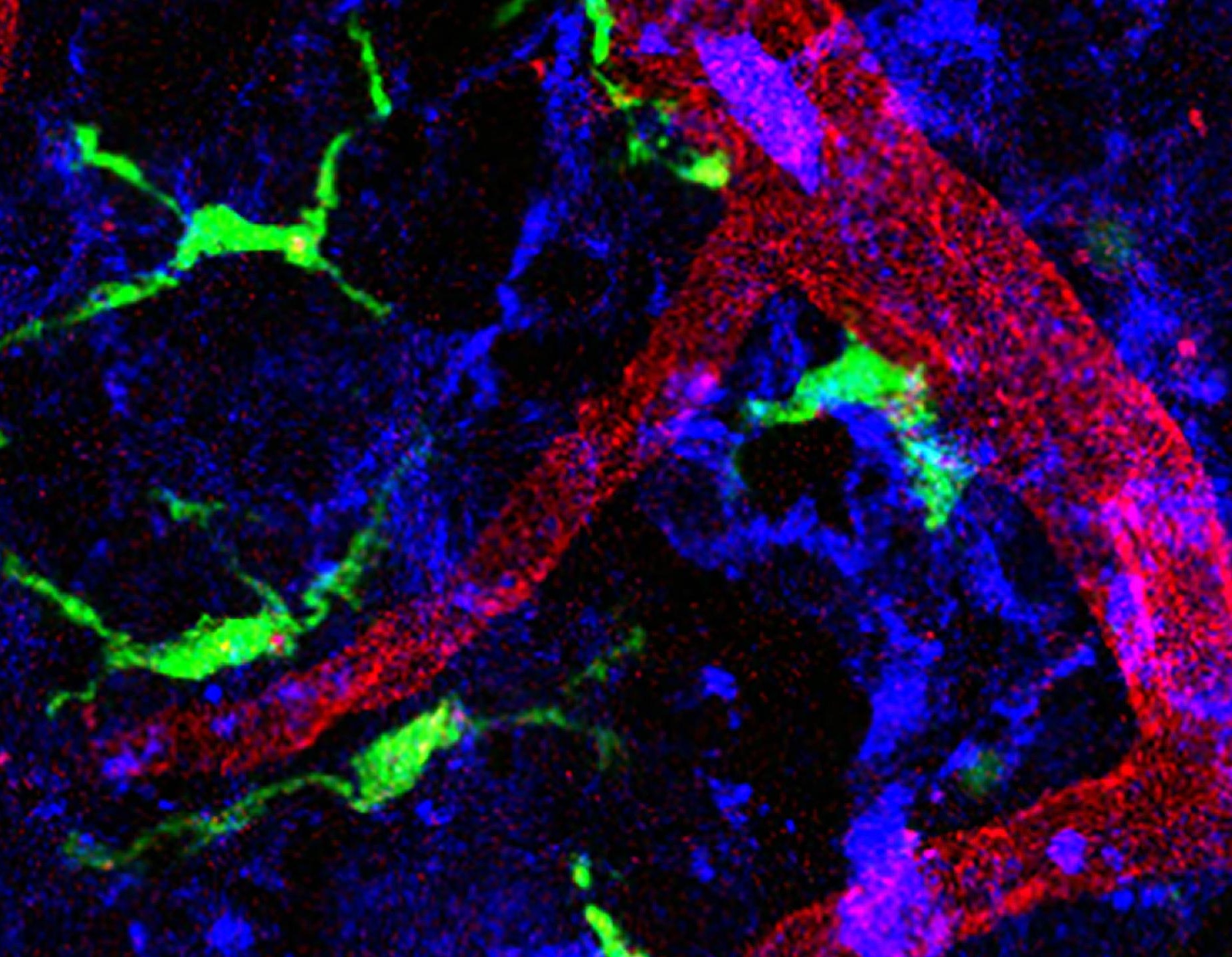

frontiers Research Topics 


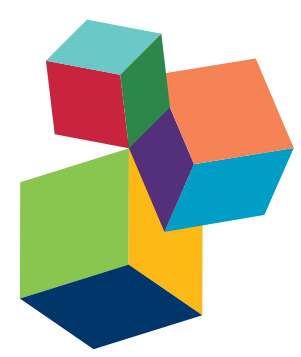

Frontiers Copyright Statement

(c) Copyright 2007-2017 Frontiers Media SA. All rights reserved.

All content included on this site, such as text, graphics, logos, button

icons, images, video/audio clips,

downloads, data compilations and

software, is the property of or is

licensed to Frontiers Media SA

("Frontiers") or its licensees and/or subcontractors. The copyright in the text of individual articles is the property of their respective authors, subject to a license granted to Frontiers.

The compilation of articles constituting

this e-book, wherever published,

as well as the compilation of all other

content on this site, is the exclusive property of Frontiers. For the

conditions for downloading and copying of e-books from Frontiers' website, please see the Terms for Website Use. If purchasing Frontiers e-books from other websites or sources, the conditions of the website concerned apply.

Images and graphics not forming part of user-contributed materials may not be downloaded or copied without permission.

Individual articles may be downloaded and reproduced in accordance with the principles of the CC-BY

licence subject to any copyright or other notices. They may not be re-sold as an e-book.

As author or other contributor you grant a CC-BY licence to others to reproduce your articles, including any graphics and third-party materials supplied by you, in accordance with the Conditions for Website Use and subject to any copyright notices which you include in connection with your articles and materials.

All copyright, and all rights therein, are protected by national and international copyright laws.

The above represents a summary only. For the full conditions see the Conditions for Authors and the Conditions for Website Use.

ISSN 1664-8714 ISBN 978-2-88945-364-1

DOI 10.3389/978-2-88945-364-1

\section{About Frontiers}

Frontiers is more than just an open-access publisher of scholarly articles: it is a pioneering approach to the world of academia, radically improving the way scholarly research is managed. The grand vision of Frontiers is a world where all people have an equal opportunity to seek, share and generate knowledge. Frontiers provides immediate and permanent online open access to all its publications, but this alone is not enough to realize our grand goals.

\section{Frontiers Journal Series}

The Frontiers Journal Series is a multi-tier and interdisciplinary set of open-access, online journals, promising a paradigm shift from the current review, selection and dissemination processes in academic publishing. All Frontiers journals are driven by researchers for researchers; therefore, they constitute a service to the scholarly community. At the same time, the Frontiers Journal Series operates on a revolutionary invention, the tiered publishing system, initially addressing specific communities of scholars, and gradually climbing up to broader public understanding, thus serving the interests of the lay society, too.

\section{Dedication to quality}

Each Frontiers article is a landmark of the highest quality, thanks to genuinely collaborative interactions between authors and review editors, who include some of the world's best academicians. Research must be certified by peers before entering a stream of knowledge that may eventually reach the public - and shape society; therefore, Frontiers only applies the most rigorous and unbiased reviews.

Frontiers revolutionizes research publishing by freely delivering the most outstanding research, evaluated with no bias from both the academic and social point of view. By applying the most advanced information technologies, Frontiers is catapulting scholarly publishing into a new generation.

\section{What are Frontiers Research Topics?}

Frontiers Research Topics are very popular trademarks of the Frontiers Journals Series: they are collections of at least ten articles, all centered on a particular subject. With their unique mix of varied contributions from Original Research to Review Articles, Frontiers Research Topics unify the most influential researchers, the latest key findings and historical advances in a hot research area! Find out more on how to host your own Frontiers Research Topic or contribute to one as an author by contacting the Frontiers Editorial Office: researchtopics@frontiersin.org 


\section{INFLAMMATION IN THE CNS: ADVANCING THE FIELD USING INTRAVITAL IMAGING}

Topic Editors:

Saparna Pai, James Cook University, The Centenary Institute and University of Sydney, Australia

Michael J. Hickey, Monash Medical Centre, Australia

Wolfgang Weninger, The Centenary Institute, University of Sydney and Royal Prince Alfred Hospital, Australia

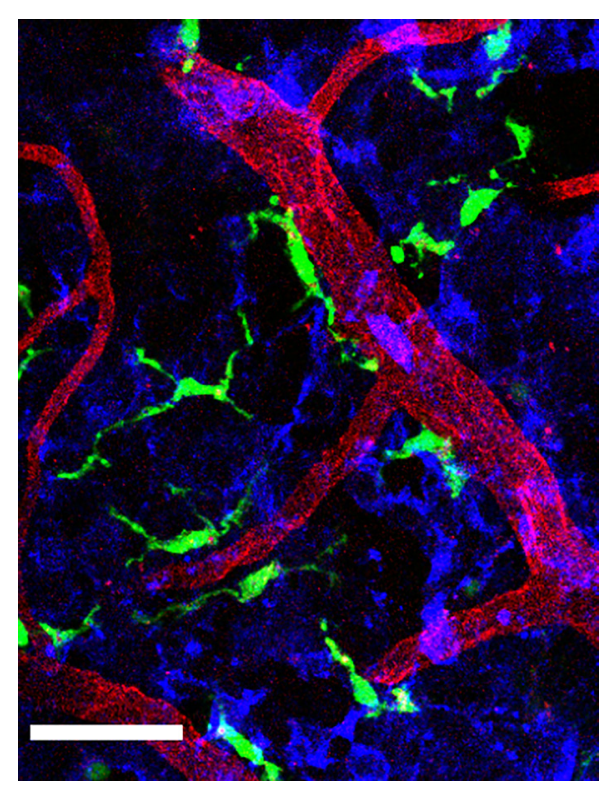

Maximum projection confocal microscopy of the top of a brain of DPE-GFP mice showing $\mathrm{CD} 163^{+}$macrophages (blue) and $\mathrm{GFP}^{+}$myeloid cells (green) distributed around a CD31+ blood vessel (red). Scale bar $58 \mu \mathrm{m}$.

Image by Saparna Pai
Inflammation of the CNS can have devastating, long-lived, and in some cases fatal consequences for patients. The stimuli that can induce CNS inflammation are diverse, and include infectious agents, autoimmune responses against CNSexpressed antigens, and sterile inflammation following ischemia or traumatic injury. In these conditions, cells of the immune system play central roles in promulgation and resolution of the inflammatory response. However, the immunological mechanisms at work in these diverse responses differ according to the nature of the response. Our understanding of the actions of immune cells in the CNS has been restricted by the difficulty in visualising leukocytes as they undergo recruitment from the cerebral microvasculature and following their entry into the CNS parenchyma. However, advances in in vivo microscopy over the last $10-15$ years have overcome many of these difficulties, and studies using these forms of microscopy have revealed a wealth of new information regarding the cellular and molecular mechanisms of CNS inflammation. 
This Research Topic brings together state of the art reviews examining the use of in vivo imaging in investigating inflammation and leukocyte behaviour in the CNS. Papers in this Research Topic describe how in vivo microscopy has increased our understanding of the actions of immune cells in the inflamed CNS, following various stimuli including autoimmunity, infection and sterile inflammation.

Citation: Pai, S., Hickey, M. J., Weninger, W., eds. (2017). Inflammation in the CNS: Advancing the Field Using Intravital Imaging. Lausanne: Frontiers Media. doi: 10.3389/978-2-88945-364-1 


\section{Table of Contents}

05 Editorial: Inflammation in the CNS: Advancing the Field Using Intravital Imaging

Saparna Pai, Michael J. Hickey and Wolfgang Weninger

07 T Cell Interstitial Migration: Motility Cues from the Inflamed Tissue for Micro- and Macro-Positioning

Alison Gaylo, Dillon C. Schrock, Ninoshka R. J. Fernandes and Deborah J. Fowell

20 Current Evidence for a Role of the Kynurenine Pathway of Tryptophan Metabolism in Multiple Sclerosis

Michael D. Lovelace, Bianca Varney, Gayathri Sundaram, Nunzio F. Franco, Mei Li Ng, Saparna Pai, Chai K. Lim, Gilles J. Guillemin and Bruce J. Brew

Nutritional and Nanotechnological Modulators of Microglia

Dusica Maysinger and Issan Zhang

Imaging of Leukocyte Trafficking in Alzheimer's Disease

Enrica Pietronigro, Elena Zenaro and Gabriela Constantin

67 Ongoing Oxidative Stress Causes Subclinical Neuronal Dysfunction in the Recovery Phase of EAE

Helena Radbruch, Daniel Bremer, Robert Guenther, Zoltan Cseresnyes, Randall Lindquist, Anja E. Hauser and Raluca Niesner

77 Analyzing Nicotinamide Adenine Dinucleotide Phosphate Oxidase Activation in Aging and Vascular Amyloid Pathology

Helena Radbruch, Ronja Mothes, Daniel Bremer, Stefanie Seifert, Ralf Köhler, Julian Pohlan, Lennard Ostendorf, Robert Günther, Ruth Leben, Werner Stenzel, Raluca Aura Niesner and Anja E. Hauser

89 Fungal Infection in the Brain: What We Learned from Intravital Imaging Meiqing Shi and Christopher H. Mody

96 Role of Tumor Necrosis Factor Superfamily in Neuroinflammation and Autoimmunity

Sandip Sonar and Girdhari Lal 


\title{
Editorial: Inflammation in the CNS: Advancing the Field Using Intravital Imaging
}

\author{
Saparna Pai ${ }^{1,2,3 *}$, Michael J. Hickey ${ }^{4}$ and Wolfgang Weninger ${ }^{2,5,6}$ \\ ${ }^{1}$ Australian Institute of Tropical Health and Medicine, James Cook University, Cairns, QLD, Australia, ${ }^{2}$ Immune Imaging \\ Program, The Centenary Institute, Newtown, NSW, Australia, ${ }^{3}$ Sydney Medical School, University of Sydney, Sydney, \\ NSW, Australia, ${ }^{4}$ Centre for Inflammatory Diseases, Monash University Department of Medicine, Monash Medical Centre, \\ Melbourne, VIC, Australia, ${ }^{5}$ Discipline of Dermatology, University of Sydney, Sydney, NSW, Australia, ${ }^{6}$ Department of \\ Dermatology, Royal Prince Alfred Hospital, Camperdown, NSW, Australia
}

Keywords: intravital imaging, leucocytes, inflammation, brain, central nervous system disorders

Editorial on the Research Topic

Inflammation in the CNS: Advancing the Field Using Intravital Imaging

Inflammation of the central nervous system (CNS) contributes to a diverse array of life-threatening and debilitating conditions. These include autoimmune conditions such as multiple sclerosis (MS), progressive degenerative conditions [Alzheimer's disease $(\mathrm{AD})$ ], sterile inflammation as occurs in stroke/cerebral ischaemia, and inflammation stemming from parasitic, fungal, viral, and bacterial infections. Whilst recent developments have led to improved outcomes in some of these conditions, most notably MS (1), there remains concerns with these approaches (2). Furthermore, there is an increasing prevalence of $\mathrm{AD}$ and stroke among the ageing population in the developed world, whilst in sub-Saharan Africa, cerebral malaria remains a major cause of mortality. These factors mandate a greater understanding of the inflammatory mechanisms in the CNS associated with these conditions. As is the case with all inflammation, inflammatory responses in the CNS involve immune cell entry/migration, complex interplay between resident and circulating immune cells, parenchymal cells, the cellular constituents of the CNS microvasculature, and alterations in immune cell function.

Intravital or in vivo imaging has been a critical tool for understanding the mechanisms of inflammation throughout the body, including in the brain (3-5). Particularly, the advent of two-photon intravital microscopy (2P-IVM) has allowed researchers to directly examine the role of multiple immune cell populations in the initiation and regulation of inflammation within the CNS. 2P-IVM has become a critical tool not only for understanding the complex interplay between the cellular components of the immune system and how they act to provide protection against infection and injury but also how the dysregulation of these processes leads to disease.

Whilst application of intravital imaging to the CNS has been technically challenging, several issues have been systematically addressed over the years to facilitate generation of high-quality four dimensional $(x, y, z, t)$ images. These advances have proved pivotal in understanding animal models of CNS inflammation such as EAE. Moreover, the combination of ongoing technical developments in imaging technologies, reporter mice, and novel fluorophores for detection of cellular signalling, in parallel with improved animal models of CNS disease, has meant that the understanding of inflammatory processes in this unique organ is better than ever before.

This Frontiers Research Topic brings together studies illustrating how imaging has advanced CNS inflammation and provides an overview of what parameters can be assessed using this approach. A key point that emerges from this collection is that intravital imaging has moved beyond simplistic descriptions of immune cell accumulation at inflamed sites-new approaches allow investigation 
of the molecular activities of these cells in situ in the CNS, in an ongoing inflammatory response, in unprecedented detail.

Activated $\mathrm{T}$ cells migrating in non-lymphoid organs play important homeostatic and pathological roles, including in the CNS. Gaylo et al. discuss the mechanisms that control $\mathrm{T}$ cell interstitial migration, emphasising that inflammation can cause changes in the composition of the extracellular milieu in which $\mathrm{T}$ cells navigate. They describe how one of the challenges of using imaging to assess $\mathrm{T}$ cell migration in the brain is the difference in the composition of the stromal and parenchymal CNS components relative to other peripheral tissues.

Lovelace et al. examine evidence linking dysregulation of the kynurenine pathway of tryptophan metabolism and the pathogenesis of MS, highlighting the role of mononuclear phagocytes in generation of neurotoxic metabolites via this pathway. Investigation of this pathway in CNS inflammation has involved a diverse range of imaging approaches as described in this article, including assessment of the blood-retinal barrier in the eye, MRI for non-invasive assessment of the blood-brain barrier, 2P-IVM for examination of $\mathrm{T}$ cell infiltration in $\mathrm{EAE}$, and correlative scanning electron microscopy for assessment of cell-cell interactions in the brain.

Maysinger and Zhang define some of the emerging questions on the immunomodulatory effects of alimentary components, gut microbiota, and nanomaterials on microglial function and activation and discuss the use of bioluminescence-based platforms for these analyses.

Pietronigro et al. examine innovative 2P-IVM-based approaches for visualisation of the progression of amyloid beta deposition and alterations in microglial behaviour in a mouse model of AD. This approach has revealed previously unrecognised actions of neutrophils in amyloid plaques in the brain.

Radbruch et al. present two studies applying advanced in vivo fluorescence lifetime imaging to examine the brain in EAE (Radbruch et al.), and ageing and amyloid-related pathology (Radbruch et al.). These studies use markers to differentiate between myeloid cells and astrocytes. Importantly, the authors illustrate the capacity of contemporary imaging approaches to move beyond simply describing cell behaviour into understanding intracellular biochemistry and signalling of specific cell

\section{REFERENCES}

1. Miller DH, Khan OA, Sheremata WA, Blumhardt LD, Rice GP, Libonati MA, et al. A controlled trial of natalizumab for relapsing multiple sclerosis. $N$ Engl J Med (2003) 348:15-23. doi:10.1056/NEJMoa020696

2. Clifford DB, Yousry TA, Major EO. A decade of natalizumab and PML: has there been a tacit transfer of risk acceptance? Mult Scler (2017) 23:934-6. doi:10.1177/1352458516670735

3. Miller MJ, Wei SH, Parker I, Cahalan MD. Two-photon imaging of lymphocyte motility and antigen response in intact lymph node. Science (2002) 296:1869-73. doi:10.1126/science.1070051

4. Mempel TR, Henrickson SE, von Andrian UH. T-cell priming by dendritic cells in lymph nodes occurs in three distinct phases. Nature (2004) 427:154-9. doi: $10.1038 /$ nature 02238 types in inflamed tissues, focussing on $\mathrm{NAD}(\mathrm{P}) \mathrm{H}$ oxidase (Nox) activity and $\mathrm{Ca}^{2+}$ signalling. In a separate article focussing on the remission phase of EAE, Radbruch et al. show that Nox activity remains elevated specifically in microglia. In contrast, under conditions of amyloid deposition, Nox activity is predominantly elevated in astrocytes (Radbruch et al.).

Fungal pathogens are an important cause of CNS pathology, particularly in immunocompromised individuals, although the mechanisms of fungal invasion of the brain are poorly understood. Shi and Mody describe the use of confocal intravital microscopy to investigate the dynamic interactions undergone by Cryptococcus neoformans in the CNS microvasculature, and the unusual nature of the neutrophil response to this infection.

Finally, Sonar and Lal provide an overview of the role of TNFSF receptor-ligand interactions in driving the pathogenesis of neuroinflammation and autoimmune disease in the CNS.

In summary, we anticipate that the collection of articles in this Frontiers Research Topic will provide researchers with a useful resource for understanding how imaging can be used to investigate the dynamics of CNS inflammation in its various forms.

\section{AUTHOR CONTRIBUTIONS}

SP: inviting contributions, handling and tracking submissions, setting timeline for research topic, acting as associate editor for selected manuscripts, inviting reviewers, coordinating with co-editors, and drafting and critically revising editorial. $\mathrm{MH}$ : inviting contributions, handling and tracking submissions, acting as associate editor for selected manuscripts, inviting reviewers, and drafting and critically revising editorial. WW: handling and tracking submissions, acting as associate editor for selected manuscripts, inviting reviewers, and drafting and critically revising editorial.

\section{ACKNOWLEDGMENTS}

The authors thank Dr. Andreas Kupz, Australian Institute of Tropical Health and Medicine, James Cook University for his input. $\mathrm{MH}$ is an National Health and Medical Research Council Senior Research Fellow (Grant ID 1042775).

5. Abtin A, Jain R, Mitchell AJ, Roediger B, Brzoska AJ, Tikoo S, et al. Perivascular macrophages mediate neutrophil recruitment during bacterial skin infection. Nat Immunol (2014) 15:45-53. doi:10.1038/ni.2769

Conflict of Interest Statement: The authors declare that the research was conducted in the absence of any commercial or financial relationships that could be construed as a potential conflict of interest.

Copyright (C) 2017 Pai, Hickey and Weninger. This is an open-access article distributed under the terms of the Creative Commons Attribution License (CC BY). The use, distribution or reproduction in other forums is permitted, provided the original author(s) or licensor are credited and that the original publication in this journal is cited, in accordance with accepted academic practice. No use, distribution or reproduction is permitted which does not comply with these terms. 


\section{OPEN ACCESS}

Edited by:

Wolfgang Weninger,

The Centenary Institute, Australia

Reviewed by:

Anna Fogdell-Hahn,

Karolinska Institutet, Sweden

Emma Harriet Wilson,

University of California Riverside,

USA

*Correspondence:

Deborah J. Fowell

deborah_fowell@urmc.rochester.edu

${ }^{t}$ Alison Gaylo and Dillon C. Schrock contributed equally to this work.

Specialty section: This article was submitted to Multiple Sclerosis and Neuroimmunology, a section of the journal Frontiers in Immunology

Received: 18 August 2016 Accepted: 29 September 2016

Published: 14 October 2016

Citation:

Gaylo A, Schrock DC, Fernandes NRJ and Fowell DJ (2016) $T$ Cell Interstitial Migration: Motility Cues from the Inflamed Tissue for Micro- and Macro-Positioning.

Front. Immunol. 7:428. doi: 10.3389/fimmu.2016.00428

\section{T Cell Interstitial Migration: Motility Cues from the Inflamed Tissue for Micro- and Macro-Positioning}

\author{
Alison Gaylo ${ }^{\dagger}$ Dillon C. Schrock ${ }^{\dagger}$ Ninoshka R. J. Fernandes and Deborah J. Fowell*
}

Department of Microbiology and Immunology, David H. Smith Center for Vaccine Biology and Immunology, Aab Institute of Biomedical Sciences, University of Rochester, Rochester, NY, USA

Effector T cells exit the inflamed vasculature into an environment shaped by tissue-specific structural configurations and inflammation-imposed extrinsic modifications. Once within interstitial spaces of non-lymphoid tissues, $\mathrm{T}$ cells migrate in an apparent random, non-directional, fashion. Efficient T cell scanning of the tissue environment is essential for successful location of infected target cells or encounter with antigen-presenting cells that activate the T cell's antimicrobial effector functions. The mechanisms of interstitial $T$ cell motility and the environmental cues that may promote or hinder efficient tissue scanning are poorly understood. The extracellular matrix (ECM) appears to play an important scaffolding role in guidance of $T$ cell migration and likely provides a platform for the display of chemotactic factors that may help to direct the positioning of $T$ cells. Here, we discuss how intravital imaging has provided insight into the motility patterns and cellular machinery that facilitates $T$ cell interstitial migration and the critical environmental factors that may optimize the efficiency of effector $T$ cell scanning of the inflamed tissue. Specifically, we highlight the local micro-positioning cues T cells encounter as they migrate within inflamed tissues, from surrounding ECM and signaling molecules, as well as a requirement for appropriate long-range macro-positioning within distinct tissue compartments or at discrete foci of infection or tissue damage. The central nervous system (CNS) responds to injury and infection by extensively remodeling the ECM and with the de novo generation of a fibroblastic reticular network that likely influences $T$ cell motility. We examine how inflammation-induced changes to the CNS landscape may regulate $T$ cell tissue exploration and modulate function.

Keywords: T cell, motility, migration, inflammation, chemokines, extracellular matrix proteins, CNS, multiphoton imaging

\section{INTRODUCTION}

The immune system's success relies on its ability to survey and rapidly respond to infection or damage throughout the body. This task depends on the efficient movement of leukocytes within and between diverse tissues. In recent years, the ability to visualize this dynamic migration using intravital imaging has led to new insights into the cellular interactions between leukocytes and the tissue stroma, T cell "search" patterns within inflamed tissues and the molecular mechanisms that control leukocyte motility and positioning (1). Innate and adaptive immune cells have distinct functional roles as part of a coordinated immune response and must move within complex tissues that are often extensively remodeled by inflammation. Therefore, it is not surprising that mechanisms of motility 
differ between immune cell types and differ for a given cell type depending on the context-dependent array of environmental cues it encounters. Here, we focus on $\mathrm{T}$ cell interstitial motility but take our "cues" from elegant studies on dendritic cell (DC) and neutrophil motility dynamics. How leukocytes integrate and interpret the cacophony of signals coming from their tissue locale into "go" signals during migration and "stop" signals for cell-cell interactions is yet to be fully understood (Figure 1). T cells must traverse their immediate tissue terrain (micro-positioning) as well as accumulate at specific focal sites of infection or damage within inflamed tissues (macro-positioning). Determining whether the cues for these related actions are shared or distinct will be critical to fully understand in situ T cell function.

The $\mathrm{T}$ cell response is initiated in lymph nodes (LNs) that drain sites of infection or inflammation. T cells are activated by antigenpresenting cells (APCs), mainly DCs, that have migrated from the infected tissue carrying pathogen-derived products presented as peptides in the context cell surface MHC molecules. The APCs also convey information on the type of pathogen or inflammation that they have encountered in peripheral tissues. Through the secretion of specific cytokines, DCs drive the differentiation of $\mathrm{T}$ cells into functionally distinct effector cells (Th1, Th2, and Th17) that are better equipped to clear specific pathogens $(2,3)$. Effector T cells also exit the LN better prepared to interact with the inflamed vasculature through upregulation of adhesion molecules and chemokine receptors (3). As reviewed elsewhere $(4,5)$, there is now a well-defined series of steps for leukocyte extravasation, the spatiotemporal kinetics of which have been greatly aided by dynamic intravital confocal and multiphoton microscopy. Once $\mathrm{T}$ cells cross the vascular and basement membrane barriers, they are met with an often chaotically organized inflamed interstitium. Effector T cells must scan and localize to the area of infection or damage to exert their effector function. Although LN-instructed tissue-specific homing cues provide some preprogramed localization bias (6-9), the inflamed endothelium appears to promote the non-selective entry of a host of different effector T cells. These effector T cells enter an inflammatory landscape unlike any tissue structure they have previously encountered and must utilize cell-intrinsic motility machinery and environment-specific cues to "explore" the new space. We know little about this process for $\mathrm{T}$ cells, but studies on innate immune cell types have revealed remarkably adaptable and coordinated mechanisms that prompt movement within inflamed tissues. DCs have been shown to be extraordinarily adept in their ability to seamlessly adapt to different adhesive substrates for locomotion enabling them to traverse a variety of inflamed microenvironments (10). For neutrophils, interstitial migration is aided by cell-cell communication, in part by neutrophil-release of leukotriene B4 (11) that facilitates collective streaming or swarming of neutrophils to a focal point of tissue damage. How effector $\mathrm{T}$ cells navigate through heterogeneous inflamed landscapes is less well-defined, yet, it is a critical final step in pathogen clearance and tissue repair.

\section{IN SITU ANALYSIS}

The mechanics of leukocyte locomotion have largely been defined using in vitro models of $2 \mathrm{D}$ and $3 \mathrm{D}$ environments, most notably collagen and fibrinogen gels and microchannels. These studies have created basic paradigms for amoeboid versus mesenchymal motility, adhesive versus non-adhesive motility $(12,13)$, the impact of physical confinement (14), and the response to soluble and immobilized chemokines (15). While useful for defining possible molecular requirements, such engineered $3 \mathrm{D}$ matrices fail to reflect the in vivo composition of the extracellular matrix (ECM), the combinatorial array of chemokinetic and chemotactic signals or the cellular diversity in a given tissue. Importantly, the in vitro models do not address the impact of inflammation on such tissue complexity. Indeed, this fundamental difference was highlighted in our recent intravital multiphoton studies of Th1 interstitial motility in the microbially inflamed dermis (16). It is widely

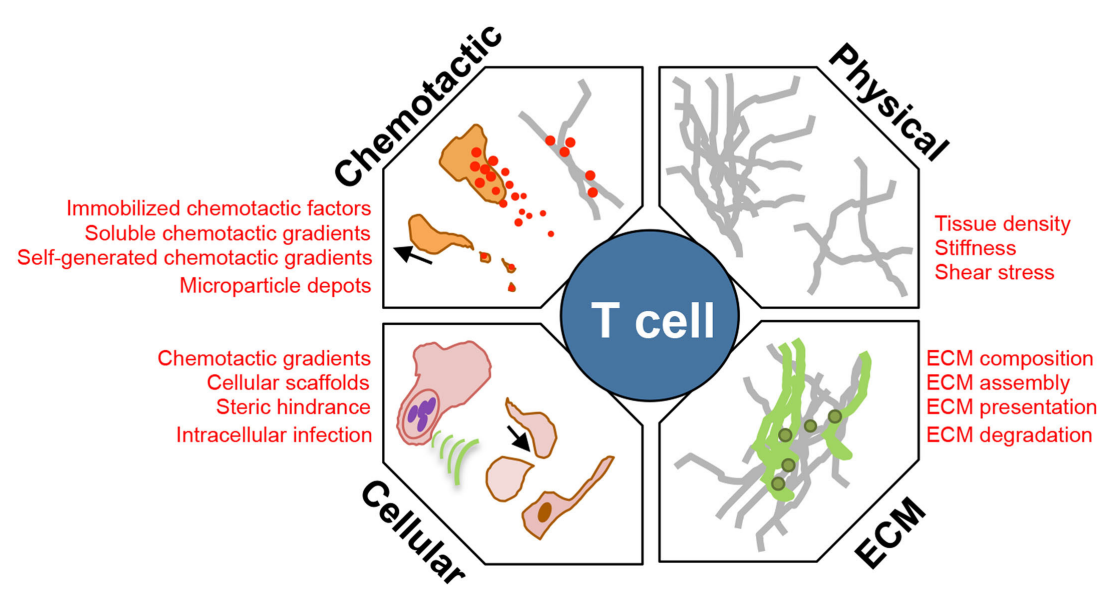

FIGURE 1 | Environmental modulators of T cell interstitial migration. T cells enter inflamed sites and must scan the interstitial tissue to locate areas of tissue damage or infection. Their efficiency of interstitial migration is influenced by: (1) the physical structure, level of confinement, and stiffness of ECM; (2) composition of the ECM, collagen fiber-associated matrix proteins, such as fibronectin; (3) cellular composition of the tissue providing: a cellular surface for traction, a source of chemotactic signal, steric hindrance, and a cellular host for pathogens that manipulate the immediate microenvironment; and (4) chemokinetic or chemotactic factors, associated with the ECM, or as a soluble gradient, or within cellular membrane fragments. 
thought that leukocyte interstitial motility in $3 \mathrm{D}$ environments is not dependent on integrin-based adhesive locomotion (12, 17-19). This notion has been supported by a number of comprehensive in vivo studies demonstrating that motility of DCs and neutrophils in the skin (steady state or acute injury), and T cells in the LN, can indeed be integrin-independent events $(11,20,21)$. In contrast, we found that Th1 cell motility closely followed the ECM fibers in the inflamed dermis and was dependent on T cell expression of the matrix-binding integrin $\alpha_{V}$ (in combination with $\beta_{1}$ and/or $\beta_{3}$ ). The discrepancy between in vitro and in vivo studies over the need for integrin-based motility likely reflects the impact of inflammation on tissue remodeling. Adjuvantinduced inflammation in the skin led to a change in the density of the collagen fibers in the dermis, in the deposition of the ECM components, and in the recruitment of innate cell types. Thus, the changes in the tissue landscape in vivo during inflammation are complex and multifactorial, and quite distinct from the simplified artificial matrices used in in vitro studies. Identifying distinct environmental components, which dictate a dependency on specific molecular machinery for motility in the context of such complexity will be important. With the growing number of intravital studies in inflamed and infected tissues, it is likely that we will continue to see challenges to the current concepts of $3 \mathrm{D}$ interstitial migration.

Intravital microscopy has proven a powerful tool for the dissection of spatiotemporal behaviors of leukocytes in situ. Numerous observations in the past 15 years have led to novel insights into immune function that had not been predicted from conventional static measures (Table 1). Yet, there are limitations to our current intravital investigative abilities in vivo. Multiphoton (two and three photon) microscopy has provided the depth resolution to begin to examine tissues in situ. However, these studies are only as good as the structures or cells that can be illuminated with fluorescent probes or with optical effects such as second-harmonic generation (SHG) or use of endogenous tissue fluorescence [elastin, keratin, $\mathrm{FAD}$, and $\mathrm{NAD}(\mathrm{P}) \mathrm{H}](22,23)$. Multiphoton constraints come from single or dual laser systems that limit the number of fluorophores that can be simultaneously excited, thus, restricting the complexity of structures and cells that can be visualized in a given field at the same time. In addition, with respect to the ECM, there is limited capacity to label these moieties in real time. Current approaches heavily rely on intravital multiphoton detection of fibrillar collagen with SHG, but this is likely only to reveal a skeleton of the ECM. Fixed tissue immune-histochemical techniques have revealed that these collagen structures are often enveloped by other matrix components, such as fibronectin and lipid moieties, which are optically silent in current multiphoton studies $(16,24,25)$. In the brain, defining the ECM structure in real time is particularly challenging as the ECM structure is often non-fibrillar and hence not visualized by SHG. Thus, dynamic imaging of leukocytes is only as good as the ability to define the optically dark "black" space surrounding the cells of interest (Figure 2). The actual matrix and/or cellular structures over or between which $\mathrm{T}$ cells move in the inflamed interstitium remain poorly defined. The ultimate goal will be to generate a topographical map of the inflamed tissue to assess the structural, chemical, and cellular contributions that act to guide
TABLE 1 | Novel insights into leukocyte function from intravital imaging.

\begin{tabular}{lc}
\hline Discovery & Reference \\
\hline Naïve T cell:APC dynamics. First look at the initiating events in & (127) \\
T cell activation showing distinct phases of short and longer & \\
interactions &
\end{tabular}

Stromal cell networks guiding T cell LN migration. Evidence that

$T$ cells utilized the fibroblastic reticular network for movement within the LN

$\mathrm{T}: \mathrm{B}$ cell dynamics at the T/B border. Motile T:B conjugates led by the $\mathrm{B}$ cells and controlled by $\mathrm{T}$ cell SAP

Lévy walks for CD8 T cells. Dynamic imaging revealed that $T$ cells migrate in a random walk pattern that may enhance search capacity for rare targets

Neutrophil swarming. Evidence that neutrophils communicate via an intercellular relay mechanism for long-range directional guidance

Lymphocyte trafficking between B cell follicles. Demonstrated that $T_{F H}$ cells move between multiple germinal centers, potentially enhancing the antibody repertoire

Intravascular leukocyte function. Neutrophils and CD8 T cells can perform antimicrobial functions without leaving the vasculature

Chemokines in T:APC interactions. CD8 T cells required expression of CXCR3 to efficiently contact and kill virally infected cells in the skin

Neutrophil trails. Neutrophils deposit chemokine-rich membrane fragments that enhance CD8 cell accumulation in the influenza-infected lung

CTL:target cell dynamics. Revealing motile kinases with targets rather than static synapses and a requirement for CTL

$(128,129)$ cooperativity

interstitial T cell scanning and positioning. Moreover, while we can intensely interrogate the micro-positioning cues measured over short distances $(200-500 \mu \mathrm{m})$ for short periods of time $2-3 \mathrm{~h}$, intravital dissection of the macro-positioning $(400-800 \mathrm{~mm})$ that likely takes place over a much longer timeframe $(8-12 \mathrm{~h})$ is challenging. Additionally, while multiphoton imaging allows for visualization of structures and cells deeper within tissues than confocal or epifluorescent imaging modalities, the resolution of multiphoton imaging is limited by long excitation wavelengths and asymmetric distortion of laser pulses, compromising intravital motility analyses such as the intracellular redistribution of molecules during migration and cell-cell interactions (26). Recent advances that combine photoactivation or photoconversion systems with multiphoton imaging will allow for pinpoint fluorescent labeling of a given cell or groups of cells in a given location in a tissue for assessment of long-range spatiotemporal dynamics, as recently shown for lymphocyte exchange between $\mathrm{B}$ cell follicles in the LN (27).

\section{LESSONS FROM LYMPHOID TISSUES}

Before entering inflamed tissues, $\mathrm{T}$ cells have undergone a series of activation events in the LN that has armed them with discrete functional properties and the ability to better respond to environmental cues that may be encountered in inflamed tissues. The spatial positioning of T cells within the LN for optimal $T$ cell activation and differentiation has been extensively reviewed 

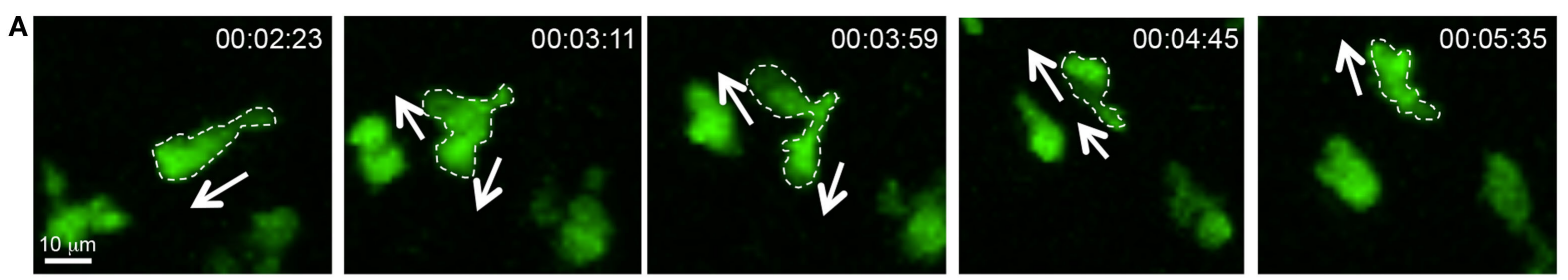

Expanding spectral detection and resolution

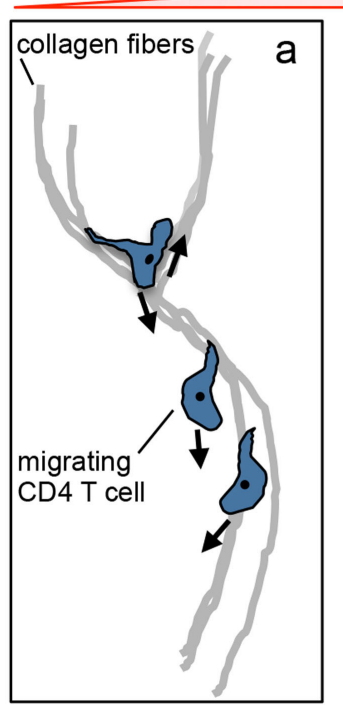

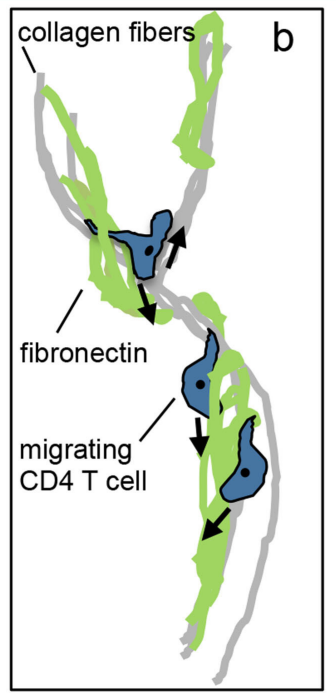
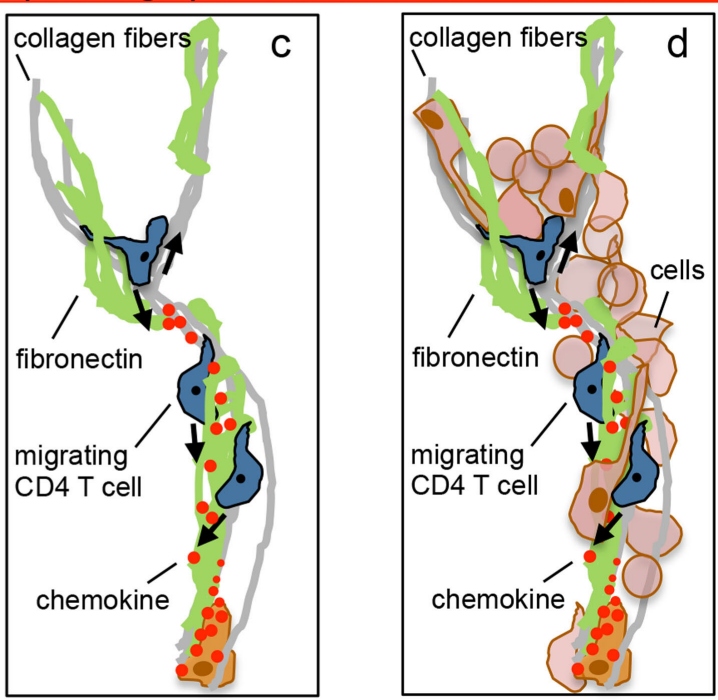

FIGURE 2 | Intravital multiphoton imaging and its limitations. (A) Motility patterns of effector CD4 T cells in the inflamed dermis showing micro-positional shape changes consistent with dynamic information sampling. (B) Imaging capabilities and limitations. Current intravital multiphoton analysis utilizes the SHG signal to highlight the fibrillar core of the tissue matrix and the association of migrating T cells with this structure (a). However, the fibrillar core is cloaked in numerous ECM proteins that cannot, at present, be visualized in real time (b). Moreover, the directional decision making is influenced by chemical signals presented on the ECM, these factors are also optically silent in current real time imaging (c). An additional layer of complexity is provided by the host of stromal and immune cells that are present in the inflamed tissue (d). Our current multiphoton capacity may allow for the detection of the SHG signal in combination with analysis of the interaction between two (may be three) additional fluorescently tagged cell types. But the ability to visualize the quality of these interactions through probes that illuminate signaling events is limited both in optically separable colors and in resolution.

elsewhere $(3,28-30)$ and is not the focus of this current review. However, there are a number of mechanistic concepts that have arisen from the study of interstitial migration of $\mathrm{T}$ cells within the LNs that are worth noting here as reference points for our discussion of T cell movement in inflamed non-lymphoid tissues. First, T cells must engage with particular DCs in order to receive activation signals for function. DCs present a variety of peptides in the context of MHC molecules on their cell surface and DCs presenting a given peptide are likely at low frequency, estimated at 1:100 (31). Thus, each antigen-specific T cell needs to scan the cell surface of many DCs before encountering one that is presenting their specific antigen. Whether this is an active "search" or an optimized chance encounter is unclear (32). Second, intravital imaging of the LN has shown that T cell amoeboid-like motility best fits a random walk with no evidence of directional migration over a $400-600 \mu \mathrm{m}$ span (33). The shaping of such motility patterns to optimize scanning of the $\mathrm{LN}$ is likely to be influenced by both $\mathrm{T}$ cell intrinsic migratory machinery and extrinsic directional cues. Third, the structural organization of the LN provides a scaffold for $\mathrm{T}$ cell migration that optimizes encounters with DCs. The highly organized fibroblastic reticular cell network (34) acts as a cellular platform for chemokine-dependent, integrin-independent, haptokinetic $\mathrm{T}$ cell movement and also promotes encounter with DCs by colocalizing T cells with DCs (35). Fourth, T cell effector functions are acquired and refined in spatially distinct locations requiring repositioning within the $\mathrm{LN}$. In recent years, our understanding of the signals for $\mathrm{T}$ cell activation and differentiation has been reshaped to incorporate location-specific instructional cues. Differentiation of both Th1 and Th2 cells in the LN is not complete without the relocation of activated $\mathrm{T}$ cells from the $\mathrm{T}$ cell zone to spatially distinct regions, namely the peri and interfollicular regions $(36,37)$. APCs in those specific regions provide additional differentiation signals to $\mathrm{T}$ cells to complete functional maturation. Chemokine production by the APCs and corresponding chemokine receptor expression by the T cells both appear key to such T cell positioning (28). How these apparent long-range positioning cues relate to the cues for the observed random walk of $\mathrm{T}$ cells still needs to be reconciled. 
The upregulation of specific chemokine receptors may make activated $\mathrm{T}$ cells more receptive to APC-derived chemotactic cues, but direct evidence of $\mathrm{T}$ cell directional migration in the $\mathrm{LN}$ toward the interfollicular region is lacking. If not actively following a chemotactic gradient, it is possible that the chemokinedriven positioning cues may instead act as focal arrest signals for randomly migrating T cells (38). Thus, observations from the LN have highlighted the importance of efficient $\mathrm{T}$ cell scanning of tissues, the interface between T cells and the tissue structure, and how tissue location can impact function: all important concepts when considering effector T cell motility in non-lymphoid tissues.

\section{MICRO- AND MACRO-POSITIONING IN INFLAMED TISSUES}

The movement of effector T cells within inflamed tissues is critical for their ability to function in the control of infection and in tissue repair. As in the $\mathrm{LN}$, T cells entering the inflamed tissue require encounter with APCs expressing their cognate ligand. The efficiency of APC encounter will depend on a balance between being able to scan a large enough area of the tissue and scanning any given area with sufficient rigor $(32,39,40)$. Unlike the LN, most inflamed non-lymphoid tissues do not appear to have an organized fibroblastic reticular cell network that could help to direct $\mathrm{T}$ cell scanning along structures that are also sites of APC localization. One might imagine that the infected tissue may require less organizational help to facilitate T:APC encounters because the frequency of both antigen-specific T cells and APCs bearing cognate antigen are enriched at the infection site in comparison to the LN. However, intravital studies in mycobacterial granulomas of the infected liver revealed that antigen presentation was surprisingly limiting (41). Antigen-specific T cells in the infected liver showed few episodes of arrest, often used as a surrogate for T:APC interactions, corresponding to poor $\mathrm{T}$ cell activation and limited IFN $\gamma$ effector function (41). Thus, there is both a conceptual and practical advantage to defining the signals that optimize T:APC encounters in inflamed tissues to enhance effector function and promote pathogen clearance. With respect to pathogen clearance, certain $\mathrm{T}$ cell cytokines have a limited range of biological activity, hence interstitial motility must also promote the correct cellular positioning relative to a region of focal infection. In the Leishmania major model of cutaneous infection, it was estimated that CD4 T cell production of IFN $\gamma$ had an 80-micron effective range, measured by the ability of secreted IFN $\gamma$ to activate nitric oxide pathways in macrophages surrounding the site of $\mathrm{T}$ cell activation (42). We suggest that the signals and mechanisms of motility employed for micro-positioning that regulate local $\mathrm{T}$ cell maneuvering of the tissue terrain on a micrometer scale may be distinct from the motility that accounts for macro-positioning of $\mathrm{T}$ cells at focal points of infection or damage on a millimeter scale (Figure 3).

\section{Motility Patterns}

In lieu of the current ability to visualize the topography of the infected tissues, researchers have initially focused on defining the patterns of $\mathrm{T}$ cell interstitial motility and the basic molecular machinery required for locomotion in inflamed tissues $(17,19$, $32,43)$. In a variety of tissue locations (skin, brain, liver, and gut), and under distinct inflammatory challenges, $\mathrm{T}$ cell interstitial motility is amoeboid-like in nature (18) (Figure 2A) and has been likened to a non-directional, random walk $(33,44)$. Unlike many innate immune cells, there is no requirement for proteolytic cleavage of tissue matrix for motility and no evidence of T:T cell communication for streaming or collective migration. A more detailed analysis of the type of random walk for CD8 T cells in the Toxoplasma gondii-infected brain revealed a pattern of random motility that most closely resembled the generalized Lévy walk (45) with a number of small steps for intense exploration of an area interspersed by random longer steps for a wider search area.

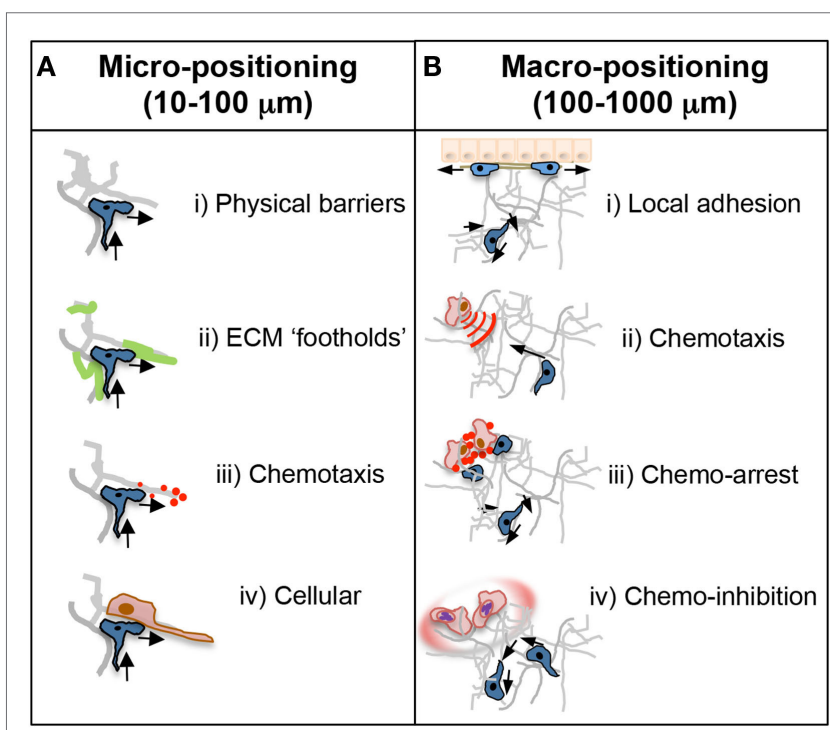

FIGURE 3 | Micro- and macro-positioning cues. (A) Micro-positioning cues for short-range cell directionality and motility patterns. T cell motility and scanning patterns are influenced by the microanatomical physical and chemical structure of the inflamed tissue. (i) The ECM could provide physical barriers to forward movement in one direction necessitating a change in direction toward a more accessible area. Cells are often seen doubling back on their path at points where the tissue may be impassable. (ii) ECM components may also positively guide directionality as an adhesive substrate that may provide footholds for $\mathrm{T}$ cell movement. (iii) Chemotactic cues, such as cytokines, are often presented on the ECM and could dictate scanning patterns and directionality. That $T$ cells often backtrack suggests chemokines may provide a chemokinetic signal in the absence of a directional cue. (iv) The cellular make up of a T cell's immediate surroundings could influence directionality through steric hindrance or by facilitating migrational paths along cellular projections closely associated with the ECM. (B) Macropositioning cues for long-range movement between distinct regions of a tissue. (i) The mechanistic basis for cellular positioning within a tissue is poorly understood in real time but is controlled in part by distinct expression of adhesion receptors and ligands on individual cell types and at distinct anatomical locations. (ii) Chemokines are also critical regulators of positioning; achieved by active chemotaxis to immobilized, or soluble diffusive gradients, or by self-generated chemotactic gradients.

(iii) Alternatively, chemokines can induce cellular arrest or retention to effectively halt $\mathrm{T}$ cells in areas of high or uniform chemoattractants such as infection foci. (iv) Not touched on in this review, but important for future study, pathogens often manipulate these chemoattractant signals through decoy receptors or soluble factors that inhibit or disrupt local directional cues for T cell positioning. 
This pattern is observed across species from sharks to honeybees in the search for rare resources. Indeed, modeling of this $\mathrm{T}$ cell behavior revealed that the Lévy walk was more efficient in finding rare APC targets than the random Brownian walk $(39,45)$. Whether this type of tissue scanning is generalizable to other $\mathrm{T}$ cell subsets and to CD8 T cells in different locations is not yet known. However, the observation has sparked much interest in examining the potential link between the T cell "search" for rare APC targets in inflamed sites to models of search theory (32). While the use of "search" evokes a desire or need that probably does not equate to $\mathrm{T}$ cell scanning of inflamed tissues for APC, such conceptual parallels are likely to provide interesting hypotheses for future studies.

\section{Mechanisms of Motility}

The movement displayed by effector T cells in lymphoid and nonlymphoid tissues morphologically resembles that of amoeboid migration with cells constantly changing shape with protruding and retracting pseudopods (Figure 2A). Amoeboid migration is driven by the forces generated by polymerization of the actomyosin cytoskeleton and, in its basic form, depends on polymerization of actin for protrusions and type II myosin-dependent actomyosin activity for contraction $(17,18,46,47)$. These forces need traction created by an interacting surface to drive locomotion. Migration on $2 \mathrm{D}$ surfaces, such as the vascular endothelium, during extravasation, requires adhesion for traction (48). Integrins are critical for this adhesion step and are dynamically activated at the leading edge via inside-out signaling from GCPRs or outside-in signaling in the presence of high substrate availability (49). Regulation of integrin de-adhesion at the trailing edge of the cells via myosin II-dependent contraction detaches the cell from its substrate allowing for forward migration (50). In 3D environments, the need for surface adhesion for motility is mitigated by the ability to use opposing surfaces of the tissue matrix for traction in an integrin-independent fashion $(14,51,52)$. Leukocytes are thought to use the matrix as a physical scaffold for pushing mediated by actin polymerization at the leading edge and myosin-dependent squeezing at the rear. This non-adhesive locomotion is dependent on the degree of physical confinement afforded by the density of the surrounding tissue matrix $(10,14,53,54)$. As previously mentioned, these lines of distinction between 2D high adhesion and 3D low adhesion modes of motility appear to become blurred in the context of inflammation, with effector CD4+ T cell interstitial motility being dependent on the matrix-binding $\alpha_{V}$ integrins (16). Integrin-dependency for interstitial migration occurred within the context of a tissue matrix that had been modified by inflammation. The inflamed dermis was associated with the fibrillar collagen scaffold (as defined by SHG) becoming less dense than the non-inflamed dermis. One possibility, therefore, is that the change in the matrix density limits the efficiency of a purely biophysical mechanism of force transduction and leads to $\mathrm{T}$ cell dependency on integrins for traction. At this stage, it is not clear if the mechanism of $\mathrm{T}$ cell interstitial motility has any effect on the efficiency of tissue scanning for cognate antigen. Interestingly, adhesion-based motility has long been thought to be a slower process than non-adhesive amoeboid movement (17, 54). Integrin-based interstitial migration may, therefore, afford the effector T cells, a more thorough scan of the local inflamed microenvironment and may enhance interactions with haptotactic signals.

\section{Intrinsic Programing for T Cell Interstitial Motility}

How micro-positioning decisions are made by $\mathrm{T}$ cells as they scan the inflamed tissue (Figure 2A) is unresolved. However, T cells are likely to be guided by their immediate physical and chemical milieu as discussed in the next section. Given, how fundamental the ability to move through inflamed tissues is to T cell function, it has been suggested that $\mathrm{T}$ cells may have specific cell-intrinsic mechanisms to optimize interstitial motility. The first demonstration in support of this notion came from the study of a hematopoietic cell-specific myosin, Myosin $1 \mathrm{~g}$ (Myo1g) (55). T cells express high levels of Myo1g, and it appears to be dynamically relocated at the plasma membrane particularly during migration in $3 \mathrm{D}$ environments. In the absence of Myolg, $\mathrm{T}$ cell 3D migration patterns were altered with increased speed and straightness. Using in silico modeling alongside in vivo imaging, the altered migration pattern in the absence of Myolg was shown to reduce the capacity of T cells to search for rare APC targets. Thus, through expression of Myolg, T cells appear to be inherently prepared for efficient scanning of $3 \mathrm{D}$ tissues. In addition to a pre-tuned program for $\mathrm{T}$ cell motility, $\mathrm{T}$ cells may also acquire new interstitial migratory potential during their activation and differentiation in the LN. Studies on the importance of integrin $\alpha_{V}$ for Th1 interstitial motility in the inflamed dermis showed that $\alpha_{V}$ expression is specifically upregulated on those activated $\mathrm{T}$ cells destined to exit the LN for effector function in peripheral tissues (16) and also enhanced in Th17 cells in the CNS (56). Therefore, the activation and differentiation process in the LN appears to prepare effector $\mathrm{T}$ cells for more efficient interactions with the inflamed matrix for interstitial motility. Distinct cytokine-producing $\mathrm{T}$ cell subsets such as Th1, Th2, and Th17 cells most likely need to function in very different inflammatory milieus that are shaped by distinct pathogen challenges. It will be interesting to determine if these cells may be distinctly programed during differentiation to express a unique motility toolbox tailored to efficient interstitial motility in specific inflammatory environments.

$\mathrm{T}$ cell-intrinsic programing for macro-positioning within a tissue is evident on numerous levels. In response to herpes simplex virus, CD4 and CD8 effector T cells both efficiently enter the infected dermis but locate to distinct regions of the skin: CD4 $\mathrm{T}$ cells to the dermis and CD8 T cells to the epidermis (57). Similar differences in the position of CD4 and CD8 T cells have been seen at other mucosal sites (57-60). Distinct macro-positioning in the skin was not due to differences in interstitial motility patterns between the two $\mathrm{T}$ cell subsets but correlated with CD8 $\mathrm{T}$ cell expression of the integrin $\alpha_{\mathrm{E}}(\mathrm{CD} 103)$ and epithelial expression of its ligand E-cadherin (57). It is not clear if there is differential $\mathrm{T}$ cell directional guidance to the epidermis, or if these interactions simply provide important stop signals for retention in the distinct areas. Similar questions arise when considering the programing differences in chemokine receptor expression between CD4 T effector cell subsets. Functionally distinct Th1, Th2, and Th17 
subsets express overlapping, yet, distinct arrays of chemokine receptors that are induced during differentiation (61). The particular chemokine receptors expressed appear to provide unique recruitment and positioning advantages to each subset. Best studied, thus far, is the expression of CXCR3 (ligands, CXCL9, CXCL10, and CXCL11) by Th1 and CD8 cells. CXCR3 is not expressed by Th2 cells and is variably expressed by Th17 cells. CXCR3 has been implicated in CD4 T cell localization to the interfollicular region in the $\mathrm{LN}$ to complete Th1 differentiation (36), Th1, and CD8 entry into numerous infected tissues and tumors (62-68) and more recently in the ability of CD8 cytotoxic $\mathrm{T}$ cells to locate and kill virally infected cells within the infected skin (69). CXCR3-deficient CD8 T cells were able to enter the vaccinia virus-infected dermis, but fewer CD8 T cells entered, or were retained in, the infection foci compared to WT CD8 T cells (69). Interestingly, of the CD8 T cells that made it into the virus-infected foci, CXCR3-deficient CD8 T cells moved more quickly than CXCR3-expressing CD8 $\mathrm{T}$ cells suggesting that CXCR3 facilitates T:APC interactions by decreasing the speed or inducing arrest of the effector T cells. Indeed, elegant studies in zebrafish have revealed that neutrophil motility is restricted in the immediate vicinity of a cellular chemokine source suggesting that, at high or uniform concentrations, chemokines can optimize retention at specific sites in the infected tissue (38). Differential chemokine receptor expression on Th1 (CXCR3+) and Th17 (CCR6+) cells also appears to play a role in autoreactive $\mathrm{T}$ cell accumulation in discrete regions of the central nervous system (CNS) resulting in quite different pathological disease (see section below). As will be discussed below, it is unclear if the expression of these chemokine receptors guide micropositioning during interstitial motility of T cells, or if the macropositioning of these distinct $\mathrm{T}$ cells is guided by focal chemokine gradients or retention signals (Figure 3 ). Nonetheless, $\mathrm{T}$ cell subset-specific chemokine receptor expression appears critical for macro-positioning within the LN and at sites of infection or inflammation. Thus, cell-intrinsic motility programs acquired by effector T cells during activation in the LN equip T cells with the potential to respond and adapt to a variety of environmental cues that may be present in the inflamed target tissue. The utilization of particular motility components within inflamed tissues will be driven by the type of inflammatory-induced changes within the target tissue.

\section{ENVIRONMENTAL CUES}

Effector T cells entering an inflamed tissue are met by a cloud of environmental cues from the ECM, lipid moieties, chemokines, cytokines, and purinergic factors, among others. How the T cell integrates and weighs the importance of the different signals for interstitial motility is not clear. On the micro-positioning level, the rapid cell shape changes during effector $\mathrm{T}$ cell interstitial motility (Figure 2A) suggest constant information sampling for directional decision-making. There is fairly sparse in vivo data on the environmental cues that actually support the motility patterns observed for $\mathrm{T}$ cell migration in $3 \mathrm{D}$, hindered in part by the difficulties in visualizing, in real time, the matrix structure and associated chemotactic factors. An emerging theme, however, is that T cells utilize the tissue structure as a scaffold for haptotaxic motility.

\section{Physical Guidance Cues}

The ECM defines the 3D structure of tissues; the organization and composition of which is distinct for individual tissues in the steady state. During inflammation, the ECM is extensively remodeled through the release of cytokines and matrix metalloproteinases, changing the biophysical structure of the matrix, its composition and its "presentation" of bioactive compounds that impact leukocyte motility and function (70-72). The interstitial matrix of many tissues is made up of a core collagen fiber network, the topography of which is shaped by its associating glycoproteins, such as fibronectin, and proteoglycans, such as decorin and versican, that contain glycosaminoglycans (GAGs) subunits. GAGs play important roles in sequestration and display of chemokines and cytokines (73-75). Leukocytes interact with the ECM and can process signals from the physical spacing and composition of the fibers, the rigidity of the matrix (mechanosensing), and immobilized chemical signaling moieties $(76,77)$. In turn, these signaling events direct leukocyte migration, function, and survival.

Intravital imaging in different tissues has shown that $\mathrm{T}$ cell motility closely follows a network of fibrillar structures defined by SHG $(16,78-80)$. As discussed for actomyosin motility, the density of the ECM is likely to dictate the effective molecular machinery that will facilitate $\mathrm{T}$ cell movement. Three-dimensional confinement studies using microchannels revealed a change in the CD8 T cell migration efficiency based solely on the spacing between fibronectin-coated surfaces, with T cell MyoIIA optimizing $\mathrm{T}$ cell motility by limiting surface adhesion (14). In vivo, within a given inflamed tissue, $\mathrm{T}$ cells are likely to experience a highly heterogeneous physical structure with variable degrees of confinement. Some have suggested that $\mathrm{T}$ cells may adapt to these changes by following paths of least resistance (17), while studies from DCs and neutrophils suggest that leukocytes can rapidly adapt to distinct terrains by switching between adhesive and non-adhesive motility $(10,11)$. The impact of utilizing possible "preferred" paths through a tissue, versus the ability to switch between migration modes, on the efficiency of $\mathrm{T}$ cell scanning of a tissue for APC encounter has yet to be determined. To add to the complexity, the actual physical space that $\mathrm{T}$ cells navigate within will also be shaped by the cellular composition of the tissue (Figure 2B), thus the true degree of $\mathrm{T}$ cell confinement in vivo is difficult to predict. In the $\mathrm{LN}, \mathrm{T}$ cells can migrate along the cellular FRC network, where fibroblasts envelop a collagen core. In non-lymphoid tissues, both fibroblasts and macrophages can extend long cellular protrusions that align along the collagen fibers and could also provide a cellular platform for $\mathrm{T}$ cell migration. Indeed, macrophage aggregates in liver mycobacterial granulomas appear to provide a cellular scaffold for effector T cell migration (81). The extent to which $\mathrm{T}$ cells interact directly with the ECM versus indirectly via ECM-associated cells is likely to be context-dependent.

In addition to changes to the structure of the interstitial matrix, inflammation and tissue damage have a dramatic impact on the composition of the ECM, with collagen fibers being decorated 
with fibronectin, vitronectin, and tenascin. The magnitude and patterning of ECM deposition likely plays a role in both $\mathrm{T}$ cell micro- and macro-positioning (Figure 3). In contexts where matrix-binding integrins facilitate $\mathrm{T}$ cell interstitial motility, the microanatomical display of the matrix ligands may guide $\mathrm{T}$ cell motility patterns by providing local "footholds" or may vary the efficiency of scanning by impacting the speed of T cell movement due to variation in traction. Effector $\mathrm{T}$ cells express a variety of matrix-binding integrins that provide ligand specificity for distinct matrix components $(49,82-84)$. The relative expression of particular matrix-binding integrins differs between inflamed tissues. In the skin, CD4 effector T cells predominantly express $\alpha_{2} \beta_{1}$ and $\alpha_{\mathrm{V}} \beta_{1} / \beta_{3}$, while in the lung and gut effector, T cells express a far wider variety of matrix-binding integrins (16). For skin and lung, the differences in integrin expression appear to correlate with the complexity of the matrix landscape. Inflammation in the skin led to a broad distribution of fibronectin across the dermis, while in the influenza-infected lung, there were spatially distinct regions that where either fibronectin-rich or collagen-rich (16). These compositionally distinct ECM regions within a tissue may afford distinct macro-positioning cues for local function or retention $(3,59,85-88)$.

Our discussion has focused on the ECM as a physical facilitator of $\mathrm{T}$ cell interstitial migration. However, studies coming from the tumor field highlight the potential barrier function of a remodeled ECM. Real-time imaging of human lung tumor slices revealed that the density and orientation of the ECM fibers surrounding the tumor mass directed $\mathrm{T}$ cell migration around the tumor but restricted them from entering the tumor mass itself (80). Recent intravital imaging of CD8 T cells in the liver demonstrated the novel ability of CD8 T cells to sample the subsinusoidal hepatocytes and kill virus-infected hepatocytes, without exiting the vasculature (89), a function that was inhibited by changes to the liver structure during fibrosis. The degree to which the ECM imposes a physical restriction on $\mathrm{T}$ cell access during chronic infection and inflammation is unclear and warrants further investigation.

\section{Chemical Guidance Cues}

Leukocytes can respond to multiple chemoattractants within the inflamed tissue including chemokines, cytokines, lipids, ECM fragments, and puronergic signals. For $\mathrm{T}$ cells, much of our understanding of chemotactic signals have come from the study of chemokines, chemokine-receptor expression, and the blockade of receptor signaling using GPCR inhibitors such as the $\mathrm{G}_{\mathrm{i}}$ inhibitor, pertussis toxin. Interference at each of these levels has reinforced that chemokines are major positioning cues for $\mathrm{T}$ cells in the steady state and during infection and inflammation (35, $61,90,91)$. While it has been assumed that diffusive chemokine gradients provide chemotactic cues for $\mathrm{T}$ cell directed migration, direct evidence for chemokine gradients on a micro- or macro-scale is limited $(38,92,93)$. A subtle directional bias was observed for migration of CD8 T cells toward HSV-1-infected cells in the skin, but cells moved away from the infection site almost as often as moving toward infected cells (94). The weak directional cues and the often observed patterns of random $\mathrm{T}$ cell motility using intravital imaging raise the possibility that chemokines direct $\mathrm{T}$ cell positioning in ways other than through classic concentration gradients (44).

For many chemokines, their activity is dependent on correct presentation by GAGs associated with the ECM or cell surfaces. While intravital studies utilizing pertussis toxin treatment have shown a dependency on $\mathrm{G}_{\mathrm{i}}$-linked signaling for interstitial migration, how the chemokines support $\mathrm{T}$ cell motility is unclear. Blockade of the CXCR3 ligand CXCL10 in the Toxoplasmainfected brain reduced the velocity of CD8 T cells but did not disrupt the Lévy walk pattern of movement in the tissue (45). Thus, chemokines may not shape the pattern of $\mathrm{T}$ cell motility but, rather, optimize the speed of interstitial migration, which may in turn increase the rate of T:APC chance encounter. This could be achieved through a basic chemokinetic mechanism or through activation of matrix-binding integrins, akin to the wellestablished role for chemokines in integrin activation and ligand binding on the vascular endothelium (95). More recently, an alternative mechanism has been proposed, that of self-generated chemotactic gradients (96). The model proposes that cells can form their own chemical gradient by degrading a local source of attractant. In vivo evidence for such a mechanism first came from studies of the migrating primordium of zebrafish where, in the presence of uniform expression of SDF-1, a signaling gradient across the primordium was achieved by sequestration of SDF-1 at the rear by the receptor CXCR7 $(97,98)$. Recent examples in the LN and spleen suggest that decoy receptors or metabolizing enzymes expressed by immune cells themselves remove or degrade the attractants to create local gradients for lymphoid migration (99-101). This potential mechanism may have distinct advantages for an effector T cell's "search" of an infected/inflamed tissue as it can be effective over a wide range of attractant concentrations enabling long-range self-directed exploration. Moreover, by regulating the expression of distinct scavenging receptors, particular effector $\mathrm{T}$ cells may separate the functionally important signals from the multitude of chemotactic signals in an inflamed tissue.

A recent study of neutrophils in influenza-infected mice reveals an additional layer of $\mathrm{T}$ cell migratory control (102). Neutrophils crawling in the interstitium of the infected trachea left long-lasting membrane fragments behind that were enriched for the chemokine CXCL12. Such chemokine depots deposited by the neutrophils appeared to provide guidance cues for incoming CD8 effector $\mathrm{T}$ cells for motility and effector function. These novel findings suggest that $\mathrm{T}$ cell interstitial migration may be shaped by the preceding recruitment and interstitial migration of innate cells that leave chemotactic trails for subsequent $\mathrm{T}$ cell movement.

The lack of evidence for macroscale directional migration leaves open the question of how chemokines guide cells to specific locations within a complex tissue. Common to many studies on $\mathrm{T}$ cell positioning is the presence of a location-specific cellular source of critical chemokines. Stromal cells in the interfollicular region of the LN were potent sources of CXCL9 required for Th1 intranodal repositioning during Th differentiation (36). Similarly, CXCL9 and CXCL10 were enriched in the vaccinia virus-infected cells in the skin and enhanced CD8 T cell positioning (69). As discussed in the context of CXCR3 expression, 
these sources of high chemokine production may, instead of being chemotactic, provide signals for $\mathrm{T}$ cell arrest and/or retention (38). Indeed, the loss of CXCR3 expression on CD8 $\mathrm{T}$ cells led to accelerated movement within the infection foci (69). Mechanistically, calcium signaling, possibly downstream of chemokine receptors, has been implicated in $\mathrm{T}$ cell arrest (103). High local concentrations of chemokines may additionally enhance inside-out activation of matrix- or cell-binding integrins, mediating strong adhesion and arrest to cellular or ECM structures (95). Furthermore, chemokines, possibly via receptor mediated tethering, have also been shown to promote $\mathrm{T}$ cell activation upon APC encounter $(104,105)$; in effect doubling down on the "stop" signal by optimizing $\mathrm{T}$ cell signaling with cognate APCs.

In conclusion, the mechanisms that facilitate efficient $\mathrm{T}$ cell interstitial motility in inflamed tissues are, in part, shaped by initial activation and differentiation events in the LN draining the site of infection or damage. The implementation of specific migratory machinery at the site of inflammation, however, appears highly context-dependent. Efficient $\mathrm{T}$ cell scanning to locate infection or damaged foci is guided by the tissue-specific matrix scaffold and optimized, in terms of speed and positioning, by chemotactic and arrest/retention cues.

\section{UNIQUE LANSCAPE OF THE CNS}

The CNS is an immunologically unique tissue and thus presents a specific set of challenges and considerations for studying $\mathrm{T}$ cell motility and positioning. In the steady state, the composition of the CNS extracellular environment is distinct from most other peripheral tissues, lacking the collagen fiber networks that often impart tissue rigidity and organ-level organization. Instead, the interstitial ECM is composed principally of long hyaluronan chains decorated with proteoglycans and cross-linked by tenascin-R (106). Along with this distinct interstitial ECM, the $\mathrm{CNS}$ is also punctuated with perineuronal nets. The perineuronal nets are distinct ECM structures composed of chondroitin sulfate proteoglycans that form dense structures around certain subsets of neurons and provide support and stability to neural connections (107). Together, the CNS ECM provides protection from mechanical stress while supporting the function of the neural network (106). Microglia, CNS-specific cells of the innate immune system, in conjunction with astrocytes, mediate tissue homeostasis and are the first to respond to tissue damage or infection (108). Surveillance of the brain and spinal cord by T cells is rare but critical for control of chronic and latent infection (109). The importance of continual immune surveillance was highlighted following reactivation of latent JC polyomavirus infection and development of a progressive multifocal leukoencephalopathy after blockade of immune extravasation using the anti- $\alpha 4$ integrin antibody Natalizumab (110). Those T cells that are present in the circulating cerebral spinal fluid under homeostatic conditions are enriched for memory $\mathrm{T}$ cell markers and CXCR3 expression (111); however, a role for specific receptors in T cell immune surveillance of the CNS has not been defined. The atypical structure of the CNS parenchyma under homeostatic conditions may require distinct mechanisms of $\mathrm{T}$ cell interstitial motility from those utilized by $\mathrm{T}$ cells recruited to the CNS by inflammation and infection (112).

During inflammation, the CNS interstitial ECM as well as the perineuronal nets undergo substantial remodeling (113). Factors produced by infiltrating immune cells and resident glial cells drive this inflammatory restructuring. Enzymes degrade the HA-rich network, and the production of new ECM components by cells such as astrocytes changes the composition of the CNS ECM, altering its mechanical properties $(106,114)$. Inflammation-induced upregulation of fibronectin and fibrillar collagens have been shown to potentiate T cell migration in the CNS (115), although it is not known if this is an integrin-dependent process. Changes in the ECM also alter its interactions with other bioactive molecules such as chemokines and cytokines. For example, the proteoglycan decorin is strongly upregulated in CNS injury (116). In other tissues, decorin has been shown to bind TGF $\beta$ and inhibit its function (117), while decorin binding to IFN $\gamma$ or TNF $\alpha$ promotes their signaling capacity (118). Alterations in these particular cytokines could change leukocyte motility by modulating responses to chemokines and altering matrix metalloproteinase activity (119). New in vivo observations on the structural changes to the CNS during inflammation have important implications for $\mathrm{T}$ cell interstitial exploration and draw parallels to other non-lymphoid and lymphoid tissues. Reports have documented the presence of reticular fiber-like structures that develop in the inflamed CNS. These structures, absent in the steady-state CNS, generate a SHG signal and are observed deep within the cortex, discrete from both the vasculature and the meninges $(79,120)$. While their molecular and cellular constituency remains unknown, the reticular fibers appear to provide a scaffold for T cell migration within the parenchyma. CD8 T cells were shown to traffic along these reticular fibers in a model of CNS infection with the protozoan parasite Toxoplasma gondii (79). Immunohistochemistry revealed a coincident fibrillar distribution of CCL21, suggesting these reticular fibers may represent rich regions of haptotactic guidance through immobilization of chemokines. As with infection in other sites, infection-induced focal chemokine production likely dictates $\mathrm{T}$ cell positioning in the CNS. West Nile virus-infected neurons produced CXCL10, which mediated CD8+ T cell parenchymal infiltration, specifically into the cerebellum (121). Intriguingly, CXCR3/CXCL10 deficiency affected parenchymal, but not perivascular $\mathrm{T}$ cell numbers, suggesting a differential requirement for this chemokine in recruitment to the CNS versus localization deeper within the tissue.

In non-infectious models of autoimmune inflammation in the brain, location-specific expression of individual chemokines appears to direct preferential CNS recruitment or retention of functionally distinct CD4 effector T cell subsets (122). Th1 effectors mediated inflammation of the parenchyma of the spinal cord, but not the brain, while Th17 effectors supported extensive parenchymal inflammation in the brain, but not the spinal cord (123-125). Not surprisingly, such differences in positioning result in distinct neurological pathologies (122). The mechanisms behind this phenomenon are incompletely understood. Differential entry sites into the CNS likely contribute to location specificity. Expression of CCL20 (a CCR6 ligand) by epithelial cells of the choroid plexus appear to promote CCR6-regulated 
entry of Th17 cells (126). Whether anatomical differences in the tissue structure or ECM composition at these locations impacts the efficiency of Th1 or Th17 migration is not yet clear. However, a recent study has revealed that Th17 cells in the CNS have elevated expression of the integrin $\alpha_{V} \beta_{3}$ (56). Expression of $\alpha_{V} \beta_{3}$ appears to facilitate integrin:ECM-driven Th17 accumulation and function in the CNS as blockade of $\alpha_{\mathrm{V}} \beta_{3}$ binding ameliorated Th17mediated EAE. Intravital imaging of Th1 and Th17 motility in the CNS will be important to determine whether they differentially utilize the inflammation-induced reticular fibers as guides for haptotactic interstitial migration and/or positional guidance.

\section{FUTURE DIRECTIONS}

Intravital imaging has only just begun to reveal the dynamic spatiotemporal control of immune function in inflamed tissues. Nonetheless, it has already provided novel insight into T cell interactions with the surrounding tissue and with other leukocytes. Future identification of critical, context-dependent, molecular regulators of cellular migration will play an important role in the development of targeted therapeutics that attenuate leukocyte function in specific immune-mediated disease. The success of these efforts will depend on the ability to understand tissue signals in a combinatorial fashion. This will require visualizing the tissue topography at the level of ECM, immobilized chemical

\section{REFERENCES}

1. Bousso P, Moreau HD. Functional immunoimaging: the revolution continues. Nat Rev Immunol (2012) 12:858-64. doi:10.1038/nri3342

2. Zhu J, Paul WE. Peripheral CD4+ T-cell differentiation regulated by networks of cytokines and transcription factors. Immunol Rev (2010) 238:247-62. doi:10.1111/j.1600-065X.2010.00951.x

3. Masopust D, Schenkel JM. The integration of T cell migration, differentiation and function. Nat Rev Immunol (2013) 13:309-20. doi:10.1038/nri3442

4. Ley K, Laudanna C, Cybulsky MI, Nourshargh S. Getting to the site of inflammation: the leukocyte adhesion cascade updated. Nat Rev Immunol (2007) 7:678-89. doi:10.1038/nri2156

5. Nourshargh S, Alon R. Leukocyte migration into inflamed tissues. Immunity (2014) 41:694-707. doi:10.1016/j.immuni.2014.10.008

6. Iwata M, Hirakiyama A, Eshima Y, Kagechika H, Kato C, Song SY. Retinoic acid imprints gut-homing specificity on T cells. Immunity (2004) 21:527-38. doi:10.1016/j.immuni.2004.08.011

7. Campbell DJ, Butcher EC. Rapid acquisition of tissue-specific homing phenotypes by $\mathrm{CD} 4(+) \mathrm{T}$ cells activated in cutaneous or mucosal lymphoid tissues. JExp Med (2002) 195:135-41. doi:10.1084/jem. 20011502

8. Sigmundsdottir H, Pan J, Debes GF, Alt C, Habtezion A, Soler D, et al. DCs metabolize sunlight-induced vitamin D3 to 'program' $\mathrm{T}$ cell attraction to the epidermal chemokine CCL27. Nat Immunol (2007) 8:285-93. doi:10.1038/ ni1433

9. Mikhak Z, Strassner JP, Luster AD. Lung dendritic cells imprint T cell lung homing and promote lung immunity through the chemokine receptor CCR4. J Exp Med (2013) 210:1855-69. doi:10.1084/jem.20130091

10. Renkawitz J, Sixt M. Mechanisms of force generation and force transmission during interstitial leukocyte migration. EMBO Rep (2010) 11:744-50. doi:10.1038/embor.2010.147

11. Lammermann T, Afonso PV, Angermann BR, Wang JM, Kastenmuller W, Parent CA, et al. Neutrophil swarms require LTB4 and integrins at sites of cell death in vivo. Nature (2013) 498:371-5. doi:10.1038/nature12175

12. Friedl P, Entschladen F, Conrad C, Niggemann B, Zanker KS. CD4+Tlymphocytes migrating in three-dimensional collagen lattices lack focal adhesions and utilize beta1 integrin-independent strategies for polarization, interaction with signals, and cellular heterogeneity as well as understanding how immune cells integrate and act on the complexity of such signals emanating from their inflamed surroundings.

\section{AUTHOR CONTRIBUTIONS}

AG and DS contributed equally. AG, DS, and DF wrote the manuscript. AG, DS, and NF researched the literature, created content, and performed experiments that informed the discussion. DF and NF designed the figures. All authors contributed to the design of the manuscript, discussion of relevant literature, and edited each draft.

\section{ACKNOWLEDGMENTS}

We thank members of the Fowell lab and members of the P01 Program for Advanced Immune Bioimaging for helpful discussion of this work.

\section{FUNDING}

This work was supported by the following awards: National Institutes of Health/National Institute of Allergy and Infectious Diseases Grants P01 AI02851 and R01 AI070826 to DF; F30 AI114036-03 to AG and T32 AI007285-28 to DS.

collagenfibersandlocomotion.EurJ Immunol(1998)28:2331-43.doi:10.1002/ (SICI)1521-4141(199808)28:08<2331::AID-IMMU2331>3.0.CO;2-C

13. Wolf K, Muller R, Borgmann S, Brocker EB, Friedl P. Amoeboid shape change and contact guidance: T-lymphocyte crawling through fibrillar collagen is independent of matrix remodeling by MMPs and other proteases. Blood (2003) 102:3262-9. doi:10.1182/blood-2002-12-3791

14. Jacobelli J, Friedman RS, Conti MA, Lennon-Dumenil AM, Piel M, Sorensen CM, et al. Confinement-optimized three-dimensional T cell amoeboid motility is modulated via myosin IIA-regulated adhesions. Nat Immunol (2010) 11:953-61. doi:10.1038/ni.1936

15. Sixt M, Lammermann T. In vitro analysis of chemotactic leukocyte migration in 3D environments. Methods Mol Biol (2011) 769:149-65. doi:10.1007/978-1-61779-207-6_11

16. Overstreet MG, Gaylo A, Angermann BR, Hughson A, Hyun YM, Lambert K, et al. Inflammation-induced interstitial migration of effector CD4(+) T cells is dependent on integrin alphaV. Nat Immunol (2013) 14:949-58. doi:10.1038/ni.2682

17. Friedl $\mathrm{P}$, Weigelin B. Interstitial leukocyte migration and immune function. Nat Immunol (2008) 9:960-9. doi:10.1038/ni.f.212

18. Lammermann T, Sixt M. Mechanical modes of 'amoeboid' cell migration. Curr Opin Cell Biol (2009) 21:636-44. doi:10.1016/j.ceb.2009.05.003

19. Lammermann T, Germain RN. The multiple faces of leukocyte interstitial migration. Semin Immunopathol (2014) 36:227-51. doi:10.1007/ s00281-014-0418-8

20. Lammermann T, Bader BL, Monkley SJ, Worbs T, Wedlich-Soldner R, Hirsch K, et al. Rapid leukocyte migration by integrin-independent flowing and squeezing. Nature (2008) 453:51-5. doi:10.1038/nature06887

21. Woolf E, Grigorova I, Sagiv A, Grabovsky V, Feigelson SW, Shulman Z, et al. Lymph node chemokines promote sustained $\mathrm{T}$ lymphocyte motility without triggering stable integrin adhesiveness in the absence of shear forces. Nat Immunol (2007) 8:1076-85. doi:10.1038/ni1499

22. Palero JA, de Bruijn HS, van der Ploeg-van den Heuvel A, Sterenborg HJ, Gerritsen HC. In vivo nonlinear spectral imaging in mouse skin. Opt Express (2006) 14:4395-402. doi:10.1364/OE.14.004395

23. Cox G, Kable E, Jones A, Fraser I, Manconi F, Gorrell MD. 3-dimensional imaging of collagen using second harmonic generation. J Struct Biol (2003) 141:53-62. doi:10.1016/S1047-8477(02)00576-2 
24. Kilarski WW, Guc E, Teo JC, Oliver SR, Lund AW, Swartz MA. Intravital immunofluorescence for visualizing the microcirculatory and immune microenvironments in the mouse ear dermis. PLoS One (2013) 8:e57135. doi:10.1371/journal.pone.0057135

25. Clark RA, Horsburgh CR, Hoffman AA, Dvorak HF, Mosesson MW, Colvin RB. Fibronectin deposition in delayed-type hypersensitivity. Reactions of normals and a patient with afibrinogenemia. J Clin Invest (1984) 74:1011-6. doi:10.1172/JCI111468

26. Hoover EE, Squier JA. Advances in multiphoton microscopy technology. Nat Photonics (2013) 7:93-101. doi:10.1038/nphoton.2012.361

27. Shulman Z, Gitlin AD, Targ S, Jankovic M, Pasqual G, Nussenzweig MC, et al. T follicular helper cell dynamics in germinal centers. Science (2013) 341:673-7. doi:10.1126/science. 1241680

28. Schulz O, Hammerschmidt SI, Moschovakis GL, Forster R. Chemokines and chemokine receptors in lymphoid tissue dynamics. Annu Rev Immunol (2016) 34:203-42. doi:10.1146/annurev-immunol-041015-055649

29. Qi H, Kastenmuller W, Germain RN. Spatiotemporal basis of innate and adaptive immunity in secondary lymphoid tissue. Annu Rev Cell Dev Biol (2014) 30:141-67. doi:10.1146/annurev-cellbio-100913-013254

30. Lian J, Luster AD. Chemokine-guided cell positioning in the lymph node orchestrates the generation of adaptive immune responses. Curr Opin Cell Biol (2015) 36:1-6. doi:10.1016/j.ceb.2015.05.003

31. Itano AA, McSorley SJ, Reinhardt RL, Ehst BD, Ingulli E, Rudensky AY, et al. Distinct dendritic cell populations sequentially present antigen to CD4 $\mathrm{T}$ cells and stimulate different aspects of cell-mediated immunity. Immunity (2003) 19:47-57. doi:10.1016/S1074-7613(03)00175-4

32. Krummel MF, Bartumeus F, Gérard A. T-cell migration, search strategies and mechanisms. Nat Rev Immunol (2016) 16:193-201. doi:10.1038/nri.2015.16

33. Cahalan MD, Parker I. Choreography of cell motility and interaction dynamics imaged by two-photon microscopy in lymphoid organs. Annu Rev Immunol (2008) 26:585-626. doi:10.1146/annurev.immunol.24.021605.090620

34. Bajenoff M, Egen JG, Koo LY, Laugier JP, Brau F, Glaichenhaus N, et al. Stromal cell networks regulate lymphocyte entry, migration, and territoriality in lymph nodes. Immunity (2006) 25:989-1001. doi:10.1016/ j.immuni.2006.10.011

35. Ebert LM, Schaerli P, Moser B. Chemokine-mediated control of T cell traffic in lymphoid and peripheral tissues. Mol Immunol (2005) 42:799-809. doi:10.1016/j.molimm.2004.06.040

36. Groom JR, Richmond J, Murooka TT, Sorensen EW, Sung JH, Bankert K, et al. CXCR3 chemokine receptor-ligand interactions in the lymph node optimize CD4+ T helper 1 cell differentiation. Immunity (2012) 37:1091-103. doi:10.1016/j.immuni.2012.08.016

37. Leon B, Ballesteros-Tato A, Browning JL, Dunn R, Randall TD, Lund FE. Regulation of $\mathrm{T}(\mathrm{H}) 2$ development by CXCR5+ dendritic cells and lymphotoxin-expressing B cells. Nat Immunol (2012) 13:681-90. doi:10.1038/ni.2309

38. Sarris M, Masson JB, Maurin D, Van der Aa LM, Boudinot P, Lortat-Jacob $\mathrm{H}$, et al. Inflammatory chemokines direct and restrict leukocyte migration within live tissues as glycan-bound gradients. Curr Biol (2012) 22:2375-82. doi:10.1016/j.cub.2012.11.018

39. Bénichou O, Loverdo C, Moreau M, Voituriez R. Intermittent search strategies. Rev Mod Phys (2011) 83:81-129. doi:10.1103/RevModPhys.83.81

40. Hills TT, Todd PM, Lazer D, Redish AD, Couzin ID; Cognitive Search Research Group. Exploration versus exploitation in space, mind, and society. Trends Cogn Sci (2015) 19:46-54. doi:10.1016/j.tics.2014.10.004

41. Egen JG, Rothfuchs AG, Feng CG, Horwitz MA, Sher A, Germain RN. Intravital imaging reveals limited antigen presentation and $\mathrm{T}$ cell effector function in mycobacterial granulomas. Immunity (2011) 34:807-19. doi:10.1016/j.immuni.2011.03.022

42. Muller AJ, Filipe-Santos O, Eberl G, Aebischer T, Spath GF, Bousso P. CD4+ $\mathrm{T}$ cells rely on a cytokine gradient to control intracellular pathogens beyond sites of antigen presentation. Immunity (2012) 37:147-57. doi:10.1016/ j.immuni.2012.05.015

43. Weninger W, Biro M, Jain R. Leukocyte migration in the interstitial space of non-lymphoid organs. Nat Rev Immunol (2014) 14:232-46. doi:10.1038/ nri3641

44. Sarris M, Sixt M. Navigating in tissue mazes: chemoattractant interpretation in complex environments. Curr Opin Cell Biol (2015) 36:93-102. doi:10.1016/j.ceb.2015.08.001
45. Harris TH, Banigan EJ, Christian DA, Konradt C, Tait Wojno ED, Norose K, et al. Generalized Levy walks and the role of chemokines in migration of effector CD8+ T cells. Nature (2012) 486:545-8. doi:10.1038/nature11098

46. Clark AG, Vignjevic DM. Modes of cancer cell invasion and the role of the microenvironment. Curr Opin Cell Biol (2015) 36:13-22. doi:10.1016/ j.ceb.2015.06.004

47. Bergert M, Chandradoss SD, Desai RA, Paluch E. Cell mechanics control rapid transitions between blebs and lamellipodia during migration. Proc Natl Acad Sci U S A (2012) 109:14434-9. doi:10.1073/pnas.1207968109

48. Chen L, Vicente-Manzanares M, Potvin-Trottier L, Wiseman PW, Horwitz AR. The integrin-ligand interaction regulates adhesion and migration through a molecular clutch. PLoS One (2012) 7:e40202. doi:10.1371/ journal.pone.0040202

49. Hynes RO. Integrins: bidirectional, allosteric signaling machines. Cell (2002) 110:673-87. doi:10.1016/S0092-8674(02)00971-6

50. Baker CM, Comrie WA, Hyun YM, Chung HL, Fedorchuk CA, Lim K, et al. Opposing roles for RhoH GTPase during T-cell migration and activation. Proc Natl Acad Sci U S A (2012) 109:10474-9. doi:10.1073/pnas.1114214109

51. Mrass P, Petravic J, Davenport MP, Weninger W. Cell-autonomous and environmental contributions to the interstitial migration of T cells. Semin Immunopathol (2010) 32:257-74. doi:10.1007/s00281-010-0212-1

52. Ruprecht V, Wieser S, Callan-Jones A, Smutny M, Morita H, Sako K, et al. Cortical contractility triggers a stochastic switch to fast amoeboid cell motility. Cell (2015) 160:673-85. doi:10.1016/j.cell.2015.01.008

53. Liu Y-J, Le Berre M, Lautenschlaeger F, Maiuri P, Callan-Jones A, Heuzé M, et al. Confinement and low adhesion induce fast amoeboid migration of slow mesenchymal cells. Cell (2015) 160:659-72. doi:10.1016/j.cell.2015.01.007

54. Jacobelli J, Bennett FC, Pandurangi P, Tooley AJ, Krummel MF. Myosin-IIA and ICAM-1 regulate the interchange between two distinct modes of T cell migration. J Immunol (2009) 182:2041-50. doi:10.4049/jimmunol.0803267

55. Gerard A, Patino-Lopez G, Beemiller P, Nambiar R, Ben-Aissa K, Liu Y, et al. Detection of rare antigen-presenting cells through $\mathrm{T}$ cell-intrinsic meandering motility, mediated by Myolg. Cell (2014) 158:492-505. doi:10.1016/ j.cell.2014.05.044

56. Du F, Garg AV, Kosar K, Majumder S, Kugler DG, Mir GH, et al. Inflammatory Th17 cells express integrin alphavbeta3 for pathogenic function. Cell Rep (2016) 16:1339-51. doi:10.1016/j.celrep.2016.06.065

57. Gebhardt T, Whitney PG, Zaid A, Mackay LK, Brooks AG, Heath WR, et al. Different patterns of peripheral migration by memory CD4+ and CD8+ T cells. Nature (2011) 477:216-9. doi:10.1038/nature10339

58. Nestle FO, Di Meglio P, Qin JZ, Nickoloff BJ. Skin immune sentinels in health and disease. Nat Rev Immunol (2009) 9:679-91. doi:10.1038/nri2622

59. Richter M, Ray SJ, Chapman TJ, Austin SJ, Rebhahn J, Mosmann TR, et al. Collagen distribution and expression of collagen-binding alphalbeta1 (VLA-1) and alpha2beta1 (VLA-2) integrins on CD4 and CD8 T cells during influenza infection. JImmunol (2007) 178:4506-16. doi:10.4049/ jimmunol.178.7.4506

60. Zhu J, Hladik F, Woodward A, Klock A, Peng T, Johnston C, et al. Persistence of HIV-1 receptor-positive cells after HSV-2 reactivation is a potential mechanism for increased HIV-1 acquisition. Nat Med (2009) 15:886-92. doi:10.1038/nm.2006

61. Griffith JW, Sokol CL, Luster AD. Chemokines and chemokine receptors: positioning cells for host defense and immunity. Annu Rev Immunol (2014) 32:659-702. doi:10.1146/annurev-immunol-032713-120145

62. Rosas LE, Barbi J, Lu B, Fujiwara Y, Gerard C, Sanders VM, et al. CXCR3-/mice mount an efficient Th1 response but fail to control Leishmania major infection. Eur J Immunol (2005) 35:515-23. doi:10.1002/eji.200425422

63. Xie JH, Nomura N, Lu M, Chen SL, Koch GE, Weng Y, et al. Antibodymediated blockade of the CXCR3 chemokine receptor results in diminished recruitment of T helper 1 cells into sites of inflammation. J Leukoc Biol (2003) 73:771-80. doi:10.1189/jlb.1102573

64. Hokeness KL, Deweerd ES, Munks MW, Lewis CA, Gladue RP, Salazar-Mather TP. CXCR3-dependent recruitment of antigen-specific T lymphocytes to the liver during murine cytomegalovirus infection. J Virol (2007) 81:1241-50. doi:10.1128/JVI.01937-06

65. Lee BJ, Giannoni F, Lyon A, Yada S, Lu B, Gerard C, et al. Role of CXCR3 in the immune response to murine gammaherpesvirus 68. J Virol (2005) 79:9351-5. doi:10.1128/JVI.79.14.9351-9355.2005 
66. Mikucki ME, Fisher DT, Matsuzaki J, Skitzki JJ, Gaulin NB, Muhitch JB, et al. Non-redundant requirement for CXCR3 signalling during tumoricidal T-cell trafficking across tumour vascular checkpoints. Nat Commun (2015) 6:7458. doi: $10.1038 /$ ncomms 8458

67. Chaturvedi V, Ertelt JM, Jiang TT, Kinder JM, Xin L, Owens KJ, et al. CXCR3 blockade protects against Listeria monocytogenes infection-induced fetal wastage. J Clin Invest (2015) 125:1713-25. doi:10.1172/JCI78578

68. Norose K, Kikumura A, Luster AD, Hunter CA, Harris TH. CXCL10 is required to maintain T-cell populations and to control parasite replication during chronic ocular toxoplasmosis. Invest Ophthalmol Vis Sci (2011) 52:389-98. doi:10.1167/iovs.10-5819

69. Hickman HD, Reynoso GV, Ngudiankama BF, Cush SS, Gibbs J, Bennink JR, et al. CXCR3 chemokine receptor enables local CD8(+) T cell migration for the destruction of virus-infected cells. Immunity (2015) 42:524-37. doi:10.1016/j.immuni.2015.02.009

70. Sorokin L. The impact of the extracellular matrix on inflammation. Nat Rev Immunol (2010) 10:712-23. doi:10.1038/nri2852

71. Hallmann R, Zhang X, Di Russo J, Li L, Song J, Hannocks MJ, et al. The regulation of immune cell trafficking by the extracellular matrix. Curr Opin Cell Biol (2015) 36:54-61. doi:10.1016/j.ceb.2015.06.006

72. Schor H, Vaday GG, Lider O. Modulation of leukocyte behavior by an inflamed extracellular matrix. Dev Immunol (2000) 7:227-38. doi:10.1155/2000/51902

73. Schumann K, Lammermann T, Bruckner M, Legler DF, Polleux J, Spatz JP, et al. Immobilized chemokine fields and soluble chemokine gradients cooperatively shape migration patterns of dendritic cells. Immunity (2010) 32:703-13. doi:10.1016/j.immuni.2010.04.017

74. Johnson Z, Proudfoot AE, Handel TM. Interaction of chemokines and glycosaminoglycans: a new twist in the regulation of chemokine function with opportunities for therapeutic intervention. Cytokine Growth Factor Rev (2005) 16:625-36. doi:10.1016/j.cytogfr.2005.04.006

75. Proudfoot AE, Handel TM, Johnson Z, Lau EK, LiWang P, Clark-Lewis I, et al. Glycosaminoglycan binding and oligomerization are essential for the in vivo activity of certain chemokines. Proc Natl Acad Sci U S A (2003) 100:1885-90. doi:10.1073/pnas.0334864100

76. Gasiorowski JZ, Murphy CJ, Nealey PF. Biophysical cues and cell behavior: the big impact of little things. Annu Rev Biomed Eng (2013) 15:155-76. doi:10.1146/annurev-bioeng-071811-150021

77. Hynes RO. The extracellular matrix: not just pretty fibrils. Science (2009) 326:1216-9. doi:10.1126/science.1176009

78. Matheu MP, Beeton C, Garcia A, Chi V, Rangaraju S, Safrina O, et al. Imaging of effector memory $\mathrm{T}$ cells during a delayed-type hypersensitivity reaction and suppression by Kv1.3 channel block. Immunity (2008) 29:602-14. doi:10.1016/j.immuni.2008.07.015

79. Wilson EH, Harris TH, Mrass P, John B, Tait ED, Wu GF, et al. Behavior of parasite-specific effector CD8+ T cells in the brain and visualization of a kinesis-associated system of reticular fibers. Immunity (2009) 30:300-11. doi:10.1016/j.immuni.2008.12.013

80. Salmon H, Franciszkiewicz K, Damotte D, Dieu-Nosjean MC, Validire P, Trautmann A, et al. Matrix architecture defines the preferential localization and migration of $\mathrm{T}$ cells into the stroma of human lung tumors. J Clin Invest (2012) 122:899-910. doi:10.1172/JCI45817

81. Egen JG, Rothfuchs AG, Feng CG, Winter N, Sher A, Germain RN. Macrophage and $\mathrm{T}$ cell dynamics during the development and disintegration of mycobacterial granulomas. Immunity (2008) 28:271-84. doi:10.1016/ j.immuni.2007.12.010

82. Zhang $\mathrm{Y}$, Wang $\mathrm{H}$. Integrin signalling and function in immune cells. Immunology (2012) 135:268-75. doi:10.1111/j.1365-2567.2011.03549.x

83. Campbell ID, Humphries MJ. Integrin structure, activation, and interactions. Cold Spring Harb Perspect Biol (2011) 3:a004994. doi:10.1101/cshperspect. a004994

84. Ruoslahti E. RGD and other recognition sequences for integrins. Annu Rev Cell Dev Biol (1996) 12:697-715. doi:10.1146/annurev.cellbio.12.1.697

85. Ray SJ, Franki SN, Pierce RH, Dimitrova S, Koteliansky V, Sprague AG, et al. The collagen binding alphalbetal integrin VLA-1 regulates CD8 $T$ cell-mediated immune protection against heterologous influenza infection. Immunity (2004) 20:167-79. doi:10.1016/S1074-7613(04)00021-4

86. Kleinschek MA, Boniface K, Sadekova S, Grein J, Murphy EE, Turner SP, et al. Circulating and gut-resident human Th17 cells express CD161 and promote intestinal inflammation. J Exp Med (2009) 206:525-34. doi:10.1084/ jem.20081712

87. Mackay LK, Stock AT, Ma JZ, Jones CM, Kent SJ, Mueller SN, et al. Long-lived epithelial immunity by tissue-resident memory T (TRM) cells in the absence of persisting local antigen presentation. Proc Natl Acad Sci U S A (2012) 109:7037-42. doi:10.1073/pnas.1202288109

88. Bergsbaken T, Bevan MJ. Proinflammatory microenvironments within the intestine regulate the differentiation of tissue-resident CD8(+) T cells responding to infection. Nat Immunol (2015) 16:406-14. doi:10.1038/ni.3108

89. Guidotti LG, Inverso D, Sironi L, Di Lucia P, Fioravanti J, Ganzer L, et al. Immunosurveillance of the liver by intravascular effector CD8(+) T cells. Cell (2015) 161:486-500. doi:10.1016/j.cell.2015.03.005

90. Thelen M, Stein JV. How chemokines invite leukocytes to dance. Nat Immunol (2008) 9:953-9. doi:10.1038/ni.f.207

91. Bromley SK, Mempel TR, Luster AD. Orchestrating the orchestrators: chemokines in control of T cell traffic. Nat Immunol (2008) 9:970-80. doi:10.1038/ni.f.213

92. Okada T, Miller MJ, Parker I, Krummel MF, Neighbors M, Hartley SB, et al. Antigen-engaged B cells undergo chemotaxis toward the T zone and form motile conjugates with helper T cells. PLoS Biol (2005) 3:e150. doi:10.1371/ journal.pbio. 0030150

93. Weber M, Hauschild R, Schwarz J, Moussion C, de Vries I, Legler DF, et al. Interstitial dendritic cell guidance by haptotactic chemokine gradients. Science (2013) 339:328-32. doi:10.1126/science.1228456

94. Ariotti S, Beltman JB, Borsje R, Hoekstra ME, Halford WP, Haanen JB, et al. Subtle CXCR3-dependent chemotaxis of CTLs within infected tissue allows efficient target localization. J Immunol (2015) 195:5285-95. doi:10.4049/ jimmunol.1500853

95. Dixit N, Simon SI. Chemokines, selectins and intracellular calcium flux: temporal and spatial cues for leukocyte arrest. Front Immunol (2012) 3:188. doi:10.3389/fimmu.2012.00188

96. Tweedy L, Susanto O, Insall RH. Self-generated chemotactic gradients-cells steering themselves. Curr Opin Cell Biol (2016) 42:46-51. doi:10.1016/ j.ceb.2016.04.003

97. Venkiteswaran G, Lewellis SW, Wang J, Reynolds E, Nicholson C, Knaut H. Generation and dynamics of an endogenous, self-generated signaling gradient across a migrating tissue. Cell (2013) 155:674-87. doi:10.1016/ j.cell.2013.09.046

98. Dona E, Barry JD, Valentin G, Quirin C, Khmelinskii A, Kunze A, et al. Directional tissue migration through a self-generated chemokine gradient. Nature (2013) 503:285-9. doi:10.1038/nature12635

99. Rot A, McKimmie C, Burt CL, Pallas KJ, Jamieson T, Pruenster M, et al. Cellautonomous regulation of neutrophil migration by the D6 chemokine decoy receptor. J Immunol (2013) 190:6450-6. doi:10.4049/jimmunol.1201429

100. Ulvmar MH, Werth K, Braun A, Kelay P, Hub E, Eller K, et al. The atypical chemokine receptor CCRL1 shapes functional CCL21 gradients in lymph nodes. Nat Immunol (2014) 15:623-30. doi:10.1038/ni.2889

101. Ramos-Perez WD, Fang V, Escalante-Alcalde D, Cammer M, Schwab SR. A map of the distribution of sphingosine 1-phosphate in the spleen. Nat Immunol (2015) 16:1245-52. doi:10.1038/ni.3296

102. Lim K, Hyun YM, Lambert-Emo K, Capece T, Bae S, Miller R, et al. Neutrophil trails guide influenza-specific CD8(+) T cells in the airways. Science (2015) 349:aaa4352. doi:10.1126/science.aaa4352

103. Bhakta NR, Oh DY, Lewis RS. Calcium oscillations regulate thymocyte motility during positive selection in the three-dimensional thymic environment. Nat Immunol (2005) 6:143-51. doi:10.1038/ni1161

104. Molon B, Gri G, Bettella M, Gomez-Mouton C, Lanzavecchia A, Martinez AC, et al. T cell costimulation by chemokine receptors. Nat Immunol (2005) 6:465-71. doi:10.1038/ni1191

105. Friedman RS, Jacobelli J, Krummel MF. Surface-bound chemokines capture and prime T cells for synapse formation. Nat Immunol (2006) 7:1101-8. doi:10.1038/ni1106-1234a

106. Lau LW, Cua R, Keough MB, Haylock-Jacobs S, Yong VW. Pathophysiology of the brain extracellular matrix: a new target for remyelination. Nat Rev Neurosci (2013) 14:722-9. doi:10.1038/nrn3550

107. Dityatev A, Schachner M, Sonderegger P. The dual role of the extracellular matrix in synaptic plasticity and homeostasis. Nat Rev Neurosci (2010) 11:735-46. doi:10.1038/nrn2898 
108. Ransohoff RM, Brown MA. Innate immunity in the central nervous system. J Clin Invest (2012) 122:1164-71. doi:10.1172/JCI58644

109. Wilson EH, Weninger W, Hunter CA. Trafficking of immune cells in the central nervous system. J Clin Invest (2010) 120:1368-79. doi:10.1172/JCI41911

110. Berger JR, Koralnik IJ. Progressive multifocal leukoencephalopathy and natalizumab - unforeseen consequences. N Engl J Med (2005) 353:414-6. doi:10.1056/NEJMe058122

111. Kivisakk P, Trebst C, Liu Z, Tucky BH, Sorensen TL, Rudick RA, et al. T-cells in the cerebrospinal fluid express a similar repertoire of inflammatory chemokine receptors in the absence or presence of CNS inflammation: implications for CNS trafficking. Clin Exp Immunol (2002) 129:510-8. doi:10.1046/j.1365-2249.2002.01947.x

112. Russo MV, McGavern DB. Immune surveillance of the CNS following infection and injury. Trends Immunol (2015) 36:637-50. doi:10.1016/ j.it.2015.08.002

113. Kwok JC, Dick G, Wang D, Fawcett JW. Extracellular matrix and perineuronal nets in CNS repair. Dev Neurobiol (2011) 71:1073-89. doi:10.1002/ dneu. 20974

114. Jones LL, Margolis RU, Tuszynski MH. The chondroitin sulfate proteoglycans neurocan, brevican, phosphacan, and versican are differentially regulated following spinal cord injury. Exp Neurol (2003) 182:399-411. doi:10.1016/ S0014-4886(03)00087-6

115. van Horssen J, Dijkstra CD, de Vries HE. The extracellular matrix in multiple sclerosis pathology. J Neurochem (2007) 103:1293-301. doi:10.1111/j.1471-4159.2007.04897.x

116. Stichel CC, Kappler J, Junghans U, Koops A, Kresse H, Muller HW. Differential expression of the small chondroitin/dermatan sulfate proteoglycans decorin and biglycan after injury of the adult rat brain. Brain Res (1995) 704:263-74. doi:10.1016/0006-8993(95)01131-5

117. Kolb M, Margetts PJ, Sime PJ, Gauldie J. Proteoglycans decorin and biglycan differentially modulate TGF-beta-mediated fibrotic responses in the lung. Am J Physiol Lung Cell Mol Physiol (2001) 280:L1327-34.

118. Bocian C, Urbanowitz AK, Owens RT, Iozzo RV, Gotte M, Seidler DG. Decorin potentiates interferon-gamma activity in a model of allergic inflammation. J Biol Chem (2013) 288:12699-711. doi:10.1074/jbc.M112.419366

119. Vaday GG, Franitza S, Schor H, Hecht I, Brill A, Cahalon L, et al. Combinatorial signals by inflammatory cytokines and chemokines mediate leukocyte interactions with extracellular matrix. J Leukoc Biol (2001) 69:885-92.

120. Herz J, Paterka M, Niesner RA, Brandt AU, Siffrin V, Leuenberger T, et al. In vivo imaging of lymphocytes in the CNS reveals different behaviour of naive T cells in health and autoimmunity. J Neuroinflammation (2011) 8:131. doi:10.1186/1742-2094-8-131

121. Zhang B, Chan YK, Lu B, Diamond MS, Klein RS. CXCR3 mediates region-specific antiviral $\mathrm{T}$ cell trafficking within the central nervous system during West Nile virus encephalitis. J Immunol (2008) 180:2641-9. doi:10.4049/jimmunol.180.4.2641
122. Pierson E, Simmons SB, Castelli L, Goverman JM. Mechanisms regulating regional localization of inflammation during CNS autoimmunity. Immunol Rev (2012) 248:205-15. doi:10.1111/j.1600-065X.2012.01126.x

123. Stromnes IM, Cerretti LM, Liggitt D, Harris RA, Goverman JM. Differential regulation of central nervous system autoimmunity by $\mathrm{T}(\mathrm{H}) 1$ and $\mathrm{T}(\mathrm{H}) 17$ cells. Nat Med (2008) 14:337-42. doi:10.1038/nm1715

124. Kroenke MA, Carlson TJ, Andjelkovic AV, Segal BM. IL-12- and IL-23modulated T cells induce distinct types of EAE based on histology, CNS chemokine profile, and response to cytokine inhibition. J Exp Med (2008) 205:1535-41. doi:10.1084/jem.20080159

125. Lees JR, Golumbek PT, Sim J, Dorsey D, Russell JH. Regional CNS responses to IFN-gamma determine lesion localization patterns during EAE pathogenesis. J Exp Med (2008) 205:2633-42. doi:10.1084/jem.20080155

126. Reboldi A, Coisne C, Baumjohann D, Benvenuto F, Bottinelli D, Lira S, et al. C-C chemokine receptor 6-regulated entry of TH-17 cells into the CNS through the choroid plexus is required for the initiation of EAE. Nat Immunol (2009) 10:514-23. doi:10.1038/ni.1716

127. Mempel TR, Henrickson SE, Von Andrian UH. T-cell priming by dendritic cells in lymph nodes occurs in three distinct phases. Nature (2004) 427(6970):154-9. doi:10.1038/nature02238

128. Allen CD, Okada T, Tang HL, Cyster JG. Imaging of germinal center selection events during affinity maturation. Science (2007) 315(5811):528-31. doi:10.1126/science. 1136736

129. Qi H, Cannons JL, Klauschen F, Schwartzberg PL, Germain RN. SAPcontrolled T-B cell interactions underlie germinal centre formation. Nature (2008) 455(7214):764-9. doi:10.1038/nature07345

130. McDonald B, Urrutia R, Yipp BG, Jenne CN, Kubes P. Intravascular neutrophil extracellular traps capture bacteria from the bloodstream during sepsis. Cell Host Microbe (2012) 12(3):324-33. doi:10.1016/j.chom.2012. 06.011

131. Halle S, Keyser KA, Stahl FR, Busche A, Marquardt A, Zheng X, et al. In vivo killing capacity of cytotoxic $\mathrm{T}$ cells is limited and involves dynamic interactions and T cell cooperativity. Immunity (2016) 44(2):233-45. doi:10.1016/j. immuni.2016.01.010

Conflict of Interest Statement: The authors declare that the research was conducted in the absence of any commercial or financial relationships that could be construed as a potential conflict of interest.

Copyright $\odot 2016$ Gaylo, Schrock, Fernandes and Fowell. This is an open-access article distributed under the terms of the Creative Commons Attribution License (CC $B Y)$. The use, distribution or reproduction in other forums is permitted, provided the original author(s) or licensor are credited and that the original publication in this journal is cited, in accordance with accepted academic practice. No use, distribution or reproduction is permitted which does not comply with these terms. 


\title{
Current Evidence for a Role of the Kynurenine Pathway of Tryptophan Metabolism in Multiple Sclerosis
}

\author{
Michael D. Lovelace ${ }^{1,2}$, Bianca Varney', Gayathri Sundaram ${ }^{1}$, Nunzio F. Franco', Mei Li Ng ${ }^{3}$, \\ Saparna Pai ${ }^{4}$, Chai K. Lim ${ }^{5}$, Gilles J. Guillemin ${ }^{5}$ and Bruce J. Brew1,2,6*
}

\begin{abstract}
'Applied Neurosciences Program, Peter Duncan Neurosciences Research Unit, St Vincent's Centre for Applied Medical Research, Sydney, NSW, Australia, ${ }^{2}$ Faculty of Medicine, St Vincent's Clinical School, University of New South Wales, Sydney, NSW, Australia, ${ }^{3}$ Faculty of Medicine, Sydney Medical School, University of Sydney, Sydney, NSW, Australia, ${ }^{4}$ Sydney Medical School, University of Sydney, Sydney, NSW, Australia, ${ }^{5}$ Neuroinflammation Group, Faculty of Medicine and Health Sciences,

Macquarie University, Sydney, NSW, Australia, ${ }^{6}$ Department of Neurology, St Vincent's Hospital, Sydney, NSW, Australia
\end{abstract}

OPEN ACCESS

Edited by:

V. Wee Yong,

University of Calgary, Canada

Reviewed by:

Francesca Fallarino,

University of Perugia, Italy

Roberta Mancuso,

Don Gnocchi Foundation

IRCCS-ONLUS, Italy

${ }^{*}$ Correspondence:

Bruce J. Brew

b.brew@unsw.edu.au

Specialty section: This article was submitted to

Multiple Sclerosis and

Neuroimmunology,

a section of the journal

Frontiers in Immunology

Received: 30 January 2016

Accepted: 10 June 2016

Published: 04 August 2016

Citation:

Lovelace MD, Varney $B$, Sundaram G, Franco NF, Ng ML, Pai S, Lim CK, Guillemin GJ and Brew BJ (2016) Current Evidence

for a Role of the Kynurenine Pathway of Tryptophan Metabolism in Multiple Sclerosis.

Front. Immunol. 7:246. doi: 10.3389/fimmu.2016.00246
The kynurenine pathway (KP) is the major metabolic pathway of the essential amino acid tryptophan (TRP). Stimulation by inflammatory molecules, such as interferon- $\gamma$ (IFN- $\gamma$ ), is the trigger for induction of the KP, driving a complex cascade of production of both neuroprotective and neurotoxic metabolites, and in turn, regulation of the immune response and responses of brain cells to the KP metabolites. Consequently, substantial evidence has accumulated over the past couple of decades that dysregulation of the KP and the production of neurotoxic metabolites are associated with many neuroinflammatory and neurodegenerative diseases, including Parkinson's disease, AIDS-related dementia, motor neurone disease, schizophrenia, Huntington's disease, and brain cancers. In the past decade, evidence of the link between the KP and multiple sclerosis (MS) has rapidly grown and has implicated the KP in MS pathogenesis. KP enzymes, indoleamine 2,3-dioxygenase (IDO-1) and tryptophan dioxygenase (highest expression in hepatic cells), are the principal enzymes triggering activation of the KP to produce kynurenine from TRP. This is in preference to other routes such as serotonin and melatonin production. In neurological disease, degradation of the blood-brain barrier, even if transient, allows the entry of blood monocytes into the brain parenchyma. Similar to microglia and macrophages, these cells are highly responsive to IFN- $\gamma$, which upregulates the expression of enzymes, including IDO-1, producing neurotoxic KP metabolites such as quinolinic acid. These metabolites circulate systemically or are released locally in the brain and can contribute to the excitotoxic death of oligodendrocytes and neurons in neurological disease principally by virtue of their agonist activity at $N$-methyl-D-aspartic acid receptors. The latest evidence is presented and discussed. The enzymes that control the checkpoints in the KP represent an attractive therapeutic target, and consequently several KP inhibitors are currently in clinical trials for other neurological diseases, and hence may make suitable candidates for MS patients. Underpinning these drug discovery endeavors, in recent years, several advances have been made in how KP metabolites are assayed in various biological fluids, and tremendous advancements have been made in how specimens are imaged to determine disease progression and involvement of various cell types and molecules in MS.

Keywords: kynurenine pathway, neuroinflammation, neurodegenerative disease, multiple sclerosis, multiphoton microscopy 


\section{RATIONALE FOR INVOLVEMENT OF KP IN MS}

Multiple sclerosis (MS) is a chronic, inflammatory demyelinating disorder of the central nervous system (CNS) whose etiology remains multifactorial and the subject of intense debate. This complexity arises from the several distinct demyelinating disorders of varying severity that are grouped under the general definition of MS. This indicates that definitive initial triggers (genetic, environmental, and others) that initiate episodes of autoimmune demyelination have yet to be identified, and that different mechanisms could contribute to lesion formation and tissue injury (1). MS is mediated by pathogenic T cells that are autoreactive against myelin antigens and coincides with broader neurodegenerative processes. Following trafficking into the brain via a compromised blood-brain barrier (BBB), $\mathrm{T}$ cells target and attack the myelin sheath of oligodendrocytes, the myelinforming cells of the CNS, which envelop central neurons and axons (2). The inflammatory plaque is the pathological hallmark of MS and can be identified using magnetic resonance imaging (MRI) or histopathologically (3). MS represents one of the most common causes of chronic neurological disabilities in young people, and its course is greatly variable (4). MS is subclassified into at least four distinguishable categories based on the course of disease. Approximately $85 \%$ of MS patients have a disease course that is marked by episodes (relapses) of neurological symptoms followed by remission periods where symptoms recover or disappear. This relapse-remitting MS form (RRMS) is often followed by secondary progressive MS (SPMS), where the disease progresses to constant neurological deterioration with no period of remission. Primary progressive MS (PPMS) affects around $10 \%$ of patients who present with gradually increasing neurological disability from the onset. Similarly, progressiverelapsing MS (PRMS), which at 5\% incidence is the rarest form, is also progressive, however, displays intermittent episodes of exacerbated symptoms. There are currently few drugs available to treat the progressive forms of disease (PPMS, SPMS, and PRMS), and therapies for RRMS have little efficacy in treating disability and neurodegeneration $(5,6)$.

Although an inflammatory aspect of the disease is clear - characterized by the presence of infiltrating macrophages and activated microglia around lesions $(7,8)$ as well as the autoimmune component arising from lymphocyte entry into the CNS, the role of other cells, such as monocytes, and other pathways that can further compromise oligodendrocyte health and contribute to the pathology of MS is increasingly being recognized by a theory of MS as a neurodegenerative disease with an autoimmune component (9). The question of whether inflammation leads to neurodegeneration or whether these are two different processes is currently unclear. The kynurenine pathway (KP) is activated in number of inflammatory and neurodegenerative diseases, including MS, and as such represents a common pathological mechanism highly relevant to our understanding of MS pathology (10). While the KP is the principal means by which tryptophan (TRP) is catabolized, it also leads to the production of several potent immunomodulatory and neuroactive intermediates, collectively called the kynurenines. Dysregulation of many of the enzymatic steps in the KP can favor the production of neurotoxic vs. neuroprotective metabolites (11). Monocytes, in particular, can be activated by high levels of inflammatory cytokines, which upregulate the expression of KP enzymes, favoring the production, and secretion of neurotoxic metabolites such as quinolinic acid (QUIN) (Figure 1, red box) (12).

Dysregulation of the KP may not be the primary cause of MS; rather the evidence thus far suggests its involvement is characterized by inflammatory episodes triggering KP activation (particularly in monocytes), trafficking to the brain, concomitant TRP degradation and production of neurotoxic metabolites. These aspects can contribute to the pathogenesis and the disease course of MS by promoting brain cell dysfunction and death, which in turn prevent the induction of essential brain cell survival and repair mechanisms. This is evidenced by studies showing that the KP is activated in early stages of MS in patients and in the experimental autoimmune encephalomyelitis (EAE) rodent model of MS (13), while monocytes are highly present in MS lesions during autoimmune episodes. Moreover, differences in disease course and clinical activity in MS are reflected by changes in the levels of KP metabolites, particularly in cerebrospinal fluid (CSF) (see CSF: A Window to Study Dysregulation of KP in the Pathology of MS and KP Metabolites Correlate with Increased MS Severity: Potential Utility as MS Biomarkers). Therefore, an activated KP could compromise the effectiveness of MS treatments, which predominantly target the autoimmune component.

In this review, we focus on evidence accumulated from MS studies that have demonstrated a dysregulation of the KP, resulting in elevated levels of neurotoxic metabolites both in the plasma and brain parenchyma (detected in CSF), and thus contribute to the progression of MS pathology. We focus on the cells that produce these damaging metabolites (Figure 1); circulating monocytes (see Peripheral Blood Monocytes as a Potent Source of Inflammatory KP Metabolites in MS) which enter the brain via a compromised BBB (see The BBB Performs a Critical Role in Maintaining Barrier Integrity and Permeability, Which Is Lost in MS) and to a lesser extent endogenous brain cells; how these metabolites are measured by analytical sampling (see CSF: A Window to Study Dysregulation of KP in the Pathology of MS) and also the mechanisms of action of these metabolites (see Mechanisms of Toxicity of KP Metabolites) and how they cause oligodendrocyte death (see Oligodendrocytes as a Target Cell for Elevated Levels of KP Metabolites in MS). We also consider how the KP can regulate both adaptive and innate immune responses (see KP Influence on the Immune System), and how some KP metabolites can serve as potential biomarkers of MS progression, detailed evidence for their association with MS (see KP Metabolites Correlate with Increased MS Severity: Potential Utility as MS Biomarkers) and therapeutic interventions (see Modulation of the KP as a Therapeutic Strategy in MS). Concurrent with this discussion, we also consider how advances in microscope imaging and allied techniques (see Microscope Imaging as a Central Tool for Advancing Knowledge of MS Pathology) have made a profound and ongoing contribution to our understanding of MS pathology. 


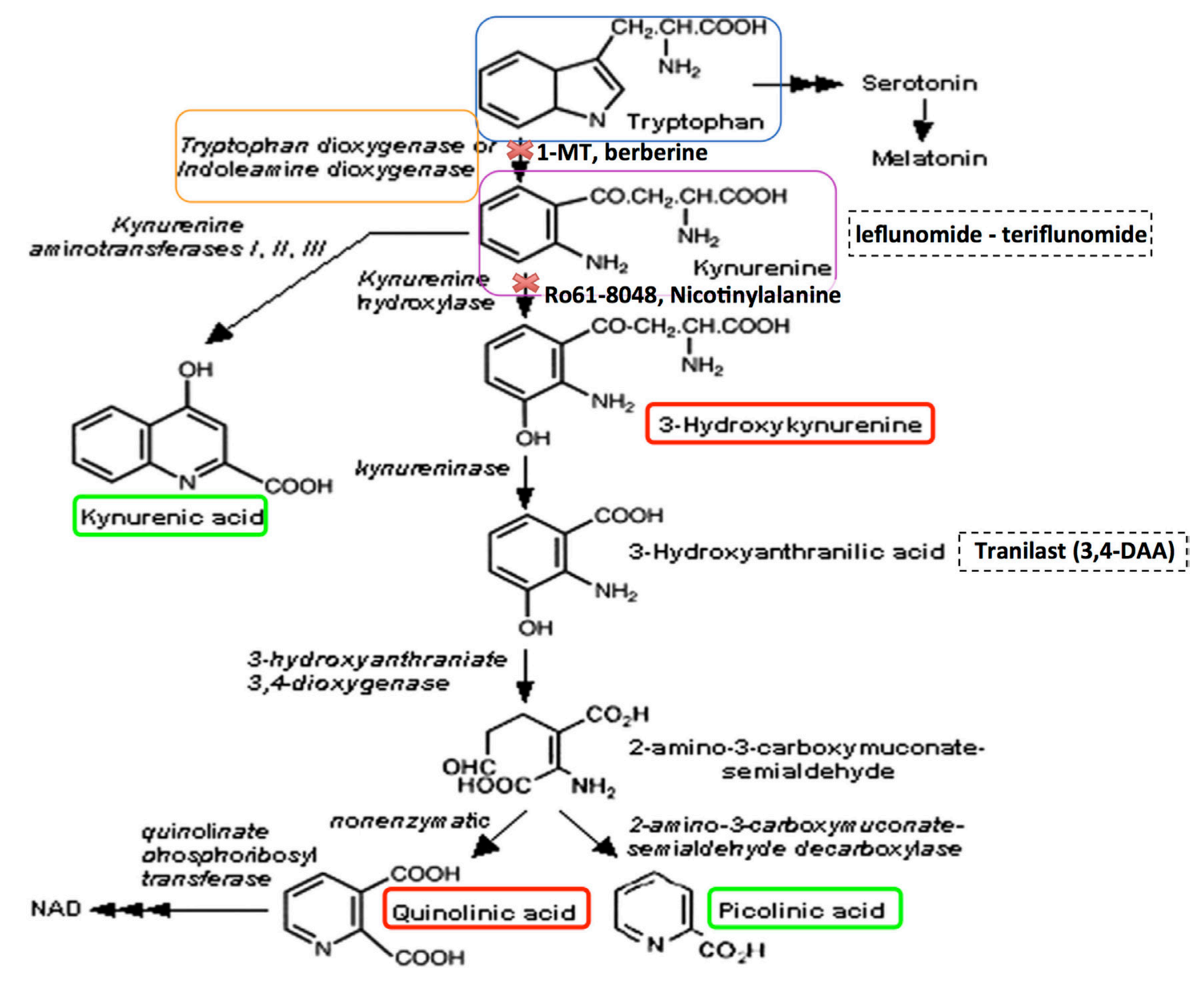

FIGURE 1 | The kynurenine pathway of tryptophan metabolism produces neuroprotective as well as neurotoxic metabolites that can influence MS pathology. Neurotoxic metabolites are circled in red, and neuroprotective metabolites in green. Tryptophan (blue box) may be metabolized to serotonin and melatonin in multi-step sequential reactions, or alternatively is metabolized via the KP. This reaction is inhibited by 1-methyl tryptophan (1-MT) or berberine. Kynurenine (purple box) is the initial rate-limiting KP product of tryptophan metabolism by the enzymes indoleamine-2,3-dioxygenase (IDO-1) and tryptophan dioxygenase (orange box). Kynurenine is then converted via kynurenine aminotransferases (KATI/II/III) to kynurenic acid, a neuroprotective molecule as it antagonizes glutamate receptor-induced neurotoxicity. 3-hydroxykynurenine is produced by further metabolism of kynurenine, for which evidence is accumulating of its neurotoxic capability. This reaction is inhibited by Ro61-8048 or nicotinylalanine. Leflunomide (Avara ${ }^{\circledR}$ ) is an immunosuppressive and anti-inflammatory drug. Teriflunomide is the active metabolite of leflunomide. These kynurenine analogs are effective in reducing active lesions in both rodent models and in a phase II clinical trial (235). Kynureninase catalyzes the conversion of 3-hydroxykynurenine to 3-hydroxyanthranilic acid. Tranilast is a synthetic anthracillic acid derivative drug with anti-inflammatory action (236). Sequential conversion to 2-amino-3-carboxymuconate-semialdehyde is the penultimate step leading to enzymatic production of (neuroprotective) picolinic acid, and the (non-enzymatic) production of the well-known neurotoxic compound quinolinic acid (QUIN). Further conversion of QUIN to the essential cofactor $\mathrm{NAD}^{+}$is catalyzed by quinolinate phosphoribosyltransferase (QPRT). Dashed boxes indicate synthetic compounds, some of which are in drug development, that are derivatives of the KP metabolite described above.

\section{THE KYNURENINE PATHWAY}

Approximately $95 \%$ of TRP is catabolized via the KP in both the CNS and periphery, which is thus the canonical route, while the remainder forms a substrate for serotonin and melatonin synthesis. In the KP, TRP is converted to $N$-formyl-L-kynurenine by indoleamine 2,3-dioxygenase (IDO-1/IDO-2) and tryptophan 2,3-dioxygenase 2 (TDO); the rate-limiting enzyme in TRP degradation. TDO is strongly and constitutively expressed in the liver; however, it is also expressed at lower levels in neurons, astrocytes, and endothelial cells $(14,15)$. Therefore, extra-hepatically, IDO-1 is the predominant enzyme in several different cell types, including monocytes, macrophages, microglia, astrocytes, neurons, and in some stem cells. IDO-2 is structurally and enzymatically similar to IDO-1; however, it is thought to function as a redundant enzyme to IDO-1 given its basal expression in a narrow range of cell types (16). Recently, new evidence suggests that IDO-2 has a role in "self-antigen" tolerance in autoimmunity and shaping immune tolerance in humans [reviewed in Ref. (17)]. Proceeding along the KP, $N$-formyl-L-kynurenine is metabolized by formamidase to L-kynurenine (KYN), the first stable intermediate metabolite (Figure 1). In the CNS, $\sim 40 \%$ of $\mathrm{KYN}$ is locally produced, whereas $60 \%$ of KYN present is absorbed from the blood (18). Kynurenine is a central KP metabolite, capable of being degraded 
through three specific pathways to generate different metabolites [kynurenic acid (KYNA), 3-hydroxykynurenine (3-HK), and anthranilic acid (AA)]. Many of the kynurenines display neuroactive properties. In particular, the neurotoxic metabolites, the $N$-methyl-D-aspartic acid (NMDA) receptor agonist and excitotoxin, QUIN, the free radical generators, 3-hydroxykynurenine (3-HK), 3-hydroxyanthranilic acid (3-HA), and the neuroprotectants, picolinic acid (PIC), and KYNA, have significant associations with disease $(19,20)$, while the essential cofactor nicotinamide adenine dinucleotide $\left(\mathrm{NAD}^{+}\right)$is a very important end metabolite produced by catabolism of QUIN by the enzyme quinolinate phosphoribosyltransferase (QPRT).

\section{TRIGGERING AGENTS OF THE KP IN MS}

Central nervous system inflammation and/or degeneration can trigger metabolism of TRP to produce kynurenine, and subsequent neurotoxic metabolites. Cells, such as monocytes and microglia, express all the KP components, whereas neurons express a restricted set and astrocytes lack expression of kynurenine 3-monoxygenase (KMO) resulting in high accumulation of kynurenine, a substrate for macrophages to further metabolize (12, 21-25). IDO-1 and TDO are the two enzymes that initiate TRP metabolism (Figure 1) and are regulated by different mechanisms. TDO is induced by corticosteroids and glucagon (26), whereas IDO-1 is induced by proinflammatory cytokines during an immune response. The inflammatory mediators that activate KP through IDO-1 induction include interferon (IFN) $-\gamma(20,27)$, interleukin (IL)-1, tumor necrosis factor (TNF)- $\alpha$ (28), cytotoxic T lymphocyte-associated antigen-4 (CTLA-4) immunoglobulin (29), toll-like receptor (TLR) (30) ligands polyinosinic:polycytidylic acid, lipopolysaccharide (LPS) (31), and unmethylated cytosine phosphatidyl guanosine (CpG) motifs (32). Although IFN- $\gamma$ is regarded as the primary inducer of IDO-1, the regulatory mechanisms of IFN- $\gamma$ mediated IDO- 1 induction can be potentiated synergistically by other proinflammatory cytokines, such as IL- 1, TNF- $\alpha$, IL- $1 \beta$, and TLR agonists, resulting in synergistic enhancement of IDO-1 expression (28, 33-36).

There is limited evidence that other enzymes within the KP can also be induced by proinflammatory cytokines, particularly by IFN- $\gamma$. Apart from the induction of IDO- 1, IFN- $\gamma$ is able to increase kynureninase (KYNU) activity in murine macrophages but not in microglial cells, which is of particular interest as TRP degradation by IDO-1 may not be the only enzymatic step controlling this pathway in activated macrophages (37). The enzyme diverting the KP toward the neurotoxic branch instead of KYNA production, KMO, is also increased through IFN- $\gamma$ in activated macrophages (38) and in the brains of immuneactivated macaques (39). Finally, in the human hippocampal progenitor cells, IL-1 $\beta$ treatment increased KMO and KYNU transcript levels (40). At millimolar concentrations, PIC acted as a macrophage coactivator by inducing macrophage inflammatory proteins $1-\alpha$ and $1-\beta$ in conjunction with IFN- $\gamma$ in the induction of reactive nitrogen intermediate production (41-43). The complex interaction between PIC and IFN- $\gamma$ highlights the importance of its involvement in inflammatory response (41) in neurodegenerative conditions. Interestingly, the current diseasemodifying agent of RRMS, IFN- $\beta 1 \mathrm{~b}$ also induces KP metabolism in human macrophages and may be a limiting factor in its efficacy in the treatment for MS (21).

\section{CSF: A WINDOW TO STUDY DYSREGULATION OF KP IN THE PATHOLOGY OF MS}

Cerebrospinal fluid bathes the inner ventricles of the cortical subventricular zone (SVZ) and subarachnoid space. CSF is a complex mixture of water, secreted proteins, enzymes, antibodies, peripheral blood, immune cells (e.g., B and T cell subsets), etc. and is constantly turned over, providing a sink for elimination of wastes from the interstitial fluid of the brain. This process is critical to proper brain homeostasis (44), and abnormal states are linked with multiple neurological diseases $(45,46)$. Choroid plexuses (47) are specialized structures located in the borders of the ventricles, facing the lateral wall, and consist of arrangements of epithelial cells (48), which secrete CSF and contain many villi which project into the ventricular space, vascular capillaries, neuronal contacts (49), and other supporting cells (Figure 2). Choroid plexuses, therefore, form a functional interface between the blood and CSF circulation and the bidirectional diffusion of important molecules, models of which are constantly evolving (50). Increasing evidence exists of alterations in choroid plexus function in various inflammatory CNS diseases (51).

From diagnostic, prognostic, and therapeutic aspects, CSF presents a unique opportunity to sample the content of fluid circulating around the brain and cerebrovascular interfaces (52-56). For many years, we have utilized CSF from patients of various neurological diseases to assay for the concentrations of KP metabolites as a discrete compartment separate from the blood plasma (for which concentrations of KP metabolites are not an accurate measure of brain levels). Our standard techniques developed and improved over the years include analysis by sensitive methods, such as high-pressure liquid chromatography (HPLC) and gas chromatography-mass spectrometry (GC-MS), to determine KP metabolite levels $(12,14,20,21,24,25,57-62)$. While analyses using these methods are extremely sensitive and able to accurately quantify KP metabolites in microliter-sized aliquots, due to BBB breakdown in MS (detailed further in Section “The BBB Performs a Critical Role in Maintaining Barrier Integrity and Permeability, Which Is Lost in MS"), the obtained data reflect the heterogeneity of such samples by influence of these factors and thus the extent of the pathology, and should be considered in concert with other pathological information. Furthermore, our previous study of brain microvascular endothelial cells (BBB endothelial cells) and pericytes uncovered that these cells express components of the $\mathrm{KP}$ that vary depending on the presence of inflammatory stimuli such as IFN- $\gamma$ or TNF- $\alpha$. After stimulation, both BBB endothelial cells and pericytes produced KYN that could potentially act as a substrate for the production of damaging KP metabolites in other neighboring cells (63). Other biomarkers within CSF related to MS pathology are also being progressively developed which would not be considered further here, e.g., Ref. (56, 64-66). 

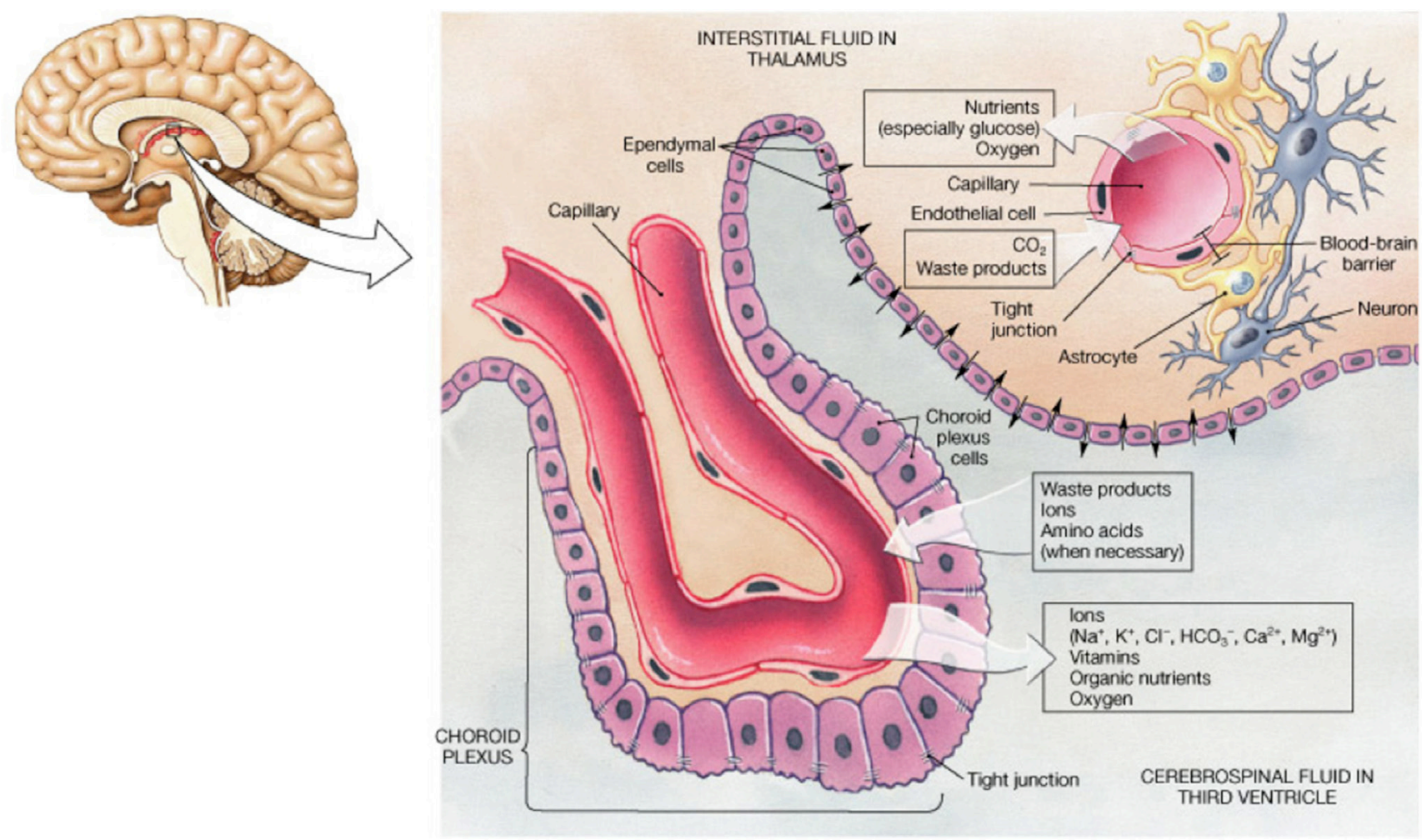

FIGURE 2 | Schematic summarizing the arrangement of cells within the choroid plexus and the physiology of exchange of solutes relevant to the understanding of how KP metabolites are found in CSF. From website http://jonlieffmd.com/blog/the-very-intelligent-choroid-plexus-epithelial-cell

\section{KP Metabolites Correlate with Increased MS Severity: Potential Utility as MS Biomarkers}

In the EAE model of MS, significantly elevated levels of QUIN and the KYN/TRP ratio in rat serum were observed and these levels correlated with increasing disease severity (67). IDO-1 expression was demonstrated to play a role in remission of acute MS in the EAE model, suggesting that the KP enzymes and metabolites could be involved in regulating disease course in MS (68). In patient samples, the first report of the potential involvement of KP activation in MS pathology was several decades ago, when decreased levels of TRP were found in plasma and CSF of MS patients (69). This involvement was confirmed with numerous subsequent studies showing alterations in KP metabolites in RRMS patients. These include evidence that patients showed decreased levels of neuroprotective KYNA in the CSF during the remission phase but became elevated during remitting, acute phases compared with healthy controls (70-72). While KYNA levels in humans are highly varying in concentration (73), an upregulation of KAT enzyme expression as a neuroprotective mechanism could be a possible explanation for this, hence more studies are needed.

Given that KP activation is modulated by proinflammatory factors including IFN- $\gamma$, it is expected that MS patients in the acute phase (compared with the later chronic neurodegenerative phase) exhibit CNS inflammation with greater involvement of $\mathrm{KP}$ metabolites in disease progression (1). A comprehensive study into the relation between several KP metabolites and neurocognitive symptoms is only now beginning to unravel the subtle distinctions in the subtypes of MS. RRMS patients show higher levels of QUIN in the relapse phase vs. during remission (74), suggesting that QUIN is a potential biomarker of active demyelination phases. Indeed, RRMS patients in remission did not show KP metabolite levels different from controls, suggesting that kynurenine dysregulation is most prominent during symptomatic periods. Interestingly, PPMS showed increased concentrations of QUIN, TRP, and KYN, whereas SPMS had decreased levels of TRP and KYNA indicating that underlying pathogenic mechanisms that occur in PPMS may be distinct from those in SPMS. Notably, PPMS and the inflammatory control group (containing inflammatory disorders of the CNS) displayed similarities with amyotrophic lateral sclerosis, supporting the working hypothesis that alterations in KP metabolites are a common pathogenic mechanism across inflammatory diseases. Importantly, Hedegaard et al. has shown that MS patients sera, and not healthy controls, contain anti-myelin basic protein (MBP) autoantibodies that facilitate IFN- $\gamma$ production (75). In line with this finding, a study examining changes in IDO-1 activity and expression in peripheral blood mononuclear cells (PBMCs) of RRMS patients found high IDO-1 expression and 
serum neopterin (a marker of inflammation) with a concomitant decrease in IFN- $\gamma$ in the relapse phase of MS, but not in the stable, remitting phase of disease (76). This suggests that inflammation and KP activation are both mechanisms that are reflected in disease relapse and appearance of clinical signs. Current treatments that aim to slow the progression of MS, including IFN- $\beta$ $1 \mathrm{a}$ and $\mathrm{1b}$ and glucocorticoids, also alter KP metabolite levels. RRMS patients treated with IFN- $\beta$ were found to have increased levels of neuroprotective KYN compared with untreated RRMS patients (77), whereas glucocorticoid treatment significantly reduced IFN- $\gamma$ levels and IDO-1 expression.

\section{THE BBB PERFORMS A CRITICAL ROLE IN MAINTAINING BARRIER INTEGRITY AND PERMEABILITY, WHICH IS LOST IN MS}

The BBB and blood-cerebrospinal fluid barrier (BCB) are complex microvasculature barriers for the CNS and systemic circulation. These barriers provide protection, nutrient, and oxygen supply to the CNS. Under physiological conditions, the $\mathrm{BBB}$ (that surrounds parenchymal venules) and $\mathrm{BCB}$ (that surrounds the choroid plexus) protect the CNS from peripheral immune cell infiltration. The tight junctions (TJ) between the endothelial cells (of the $\mathrm{BBB}$ ) and epithelial cells (of the BCB) restrict access of circulating cells to the CNS. Nevertheless, even in healthy brains, $\mathrm{T}$ cells can carry out immune surveillance of the CNS because they express adhesion molecules, chemokine receptors, and integrins that allow them to cross these barriers (78). On the other hand, the non-CNS targeted T cells are also capable of altering permeability and glial cell activity. Previous studies have shown that ovalbumin (OVA)-specific T cells are able to disrupt barrier integrity of the brain (79) and retina (80). Using an MRI approach, another group investigated in vivo $\mathrm{T}$ cell transmigration in relation to the $\mathrm{BBB}$ disruption on $\mathrm{CNS}$ tissues in a model using OVA- and proteolipid protein (PLP)-specific $\mathrm{T}$ cells, finding that antigen specificity (and not absolute number of infiltrating cells) is a critical determinant of the extent of BBB breakdown (81). Cerebral microvascular endothelial cells are also joined by TJ complexes with associated pericytes and astrocyte processes. During MS, damage of TJ proteins facilitates leukocyte infiltration, leading to oligodendrocyte death, axonal damage, demyelination, and lesion formation. Glial cell activation and further leukocyte invasion cause myelin damage and axonal degradation. Production of cytokines worsens BBB damage leading to progressive disability (82).

The CP (Figure 2) is a highly vascularized brain structure located within brain ventricles and consists of an epithelial layer forming a tight $\mathrm{BCB}$, surrounding a core of fenestrated capillaries and connective tissues. The fenestrated capillaries are surrounded by CP epithelial cells, which confer tight barrier properties and restrict the entry of immune cells in the CSF. A local impairment of the endothelial cells and chemokine production results in a reduction in barrier integrity of the $\mathrm{BCB}$, which are prominent events for early invasion of immune cells into the CSF. Indeed, the presence of oligoclonal bands from B cells in Western blots from CSF is seen in $\sim 90 \%$ of MS patients and is a common test for confirmation of MS diagnosis (52). After passing the BCB, CSF-infiltrated leukocytes produce large amount of cytokines and activate endothelial cells of the brain vasculature, inducing expression of adhesion molecules and chemokines, leading to the formation of inflammatory lesions (83).

\section{BBB Breakdown and Entry of Immune Cells as a Key Hallmark of Early MS Pathology}

A damaged BBB allows infiltration of autoreactive $\mathrm{T}$ cells and monocytes into the brain parenchyma. In MS, leukocyte infiltration into the CNS parenchyma is one of the earliest hallmarks (80, 84 ) and is thought to play a fundamental role in the development of the disease, including contributing to the early stages of lesion formation. BBB breakdown is also found in relapses (85-87); indeed, optic neuritis $(\mathrm{ON})$ was the condition in which this was first noticed. Examination of the whole-mount retina preparation from EAE Lewis rats visualized the presence of BBB breakdown, cellular infiltration, and microglial activation as the earliest abnormal events in ON. This study also correlated the intensity of the immune response with the number of infiltrated leukocytes and microglial activation in the retinal parenchyma (84). Inflammatory cells are found to predominantly colocalize within the disrupted $\mathrm{BBB}(88,89)$. Activated inflammatory cells $\left(\mathrm{ED} 1^{+}\right.$ monocytes, $\mathrm{CD}^{+}$, and $\mathrm{CD} 8^{+} \mathrm{T}$ cells) in the lumen of affected vessels are capable of disrupting BBB permeability (84). Overall, these studies emphasize that abnormal BBB permeability and leukocyte infiltration in the CNS are key events leading to pathogenesis of MS. As discussed in greater detail in Section "Peripheral Blood Monocytes as a Potent Source of Inflammatory KP Metabolites in MS," high expression of KP enzymes in activated monocytes, and their translocation into the brain parenchyma means they are poised to exert a profound impact on the survival of oligodendrocytes and neurons, and hasten the progression of lesion development and MS pathology.

Although these previous studies have provided ample evidence that BBB breakdown is the earliest event leading to MS pathology, these findings have been reinforced by modern MRI of the brain. For example, serial MRI studies have demonstrated that abnormalities of the BBB may precede myelin damage and leukocyte infiltration (90). Histopathological (91-94) and serial MRI $(90,95)$ studies indicated that structural changes may precede myelin damage and leukocyte infiltration. Cramer and colleagues use a sensitive dynamic contrast-enhanced (DCE) MRI concluded the importance of a BBB defect in MS. MS lesions are predominantly located in the periventricular normal appearing white matter $(96,97)$. The presence of $\mathrm{ON}$ and lesions can be predictive of MS progression, as an MRI-based risk stratification showed that patients with MRI T2 lesions and ON progressing faster to full MS compared with patients with ON alone (56 vs. 22\%) (98).

Subsequently, Cramer and colleagues showed that the BBB permeability is able to predict conversion from ON to MS within 2 years, in a group of patients presenting with monosymptomatic 
$\mathrm{ON}$ - and T2-lesion count compared with another group presented with T2-lesion count alone (99). Subtle disruption to the BBB is often found at discrete locations in the brain, for which modern radiographical imaging modalities such as MRI can be utilized (100). Kawakami and Flugel also used intravital two-photon imaging to measure BBB permeability. Using this imaging technique, they examined infiltration of autoreactive $\mathrm{T}$ cells across the intraluminal surface of CNS blood vessels in animal model of MS (101).

\section{KP INFLUENCE ON THE IMMUNE SYSTEM}

One of the most profound roles of the KP has been its implication in the pathological regulation of both the innate and adaptive the immune system $(18,102)$. IDO-1 is considered the major contributor to the immunoregulatory functions of the KP due to the depletion of TRP and the production of kynurenine metabolites. IDO- 1 is expressed in several types of immune cells, including microglia, monocytes, and macrophages, and can be readily induced by interferons, most effectively by IFN- $\gamma$ (102). IDO-1 activation has potent antimicrobial effects, which occurs partly through the depletion of the essential amino acid TRP. IDO-1 plays a vital function in maintaining polymorphonuclear cells effector function against pathogens (103). Conversely, IDO-1 can also suppress the immune response leading to immunological tolerance, which mediates various phenomena such as allograft acceptance, tumor camouflage, and maternofetal tolerance (18).

To date, at least three mechanisms that initiate immunological suppression are known. These immunosuppressive effects all correspond to IDO-1 activation and its downstream effects in certain populations of $\mathrm{T}$ cells. First, TRP levels are depleted following IDO-1 induction, which inhibits the proliferation of reactive $\mathrm{T}$ lymphocytes and increases their susceptibility to apoptosis (104). Second, the resulting increase in kynurenine metabolites (KYN, QUIN, and 3-HAA) interferes with proliferation and initiates selective apoptosis of $\mathrm{T}$ helper $1\left(\mathrm{~T}_{\mathrm{H}} 1\right)$ lymphocytes, which are responders to antigen-presenting cells $(105,106)$. Notably, there is a preferential inhibition for $\mathrm{T}_{\mathrm{H}} 1$ cells by IDO- 1 activation, although the activation of regulatory T-cells may also impede $\mathrm{T}_{\mathrm{H}} 2$ cells (102). There are controversial data surrounding QUIN's effect on $\mathrm{T}$ cell regulation, although it is currently thought that this process relies on a TRP-deficient microenvironment $(105,107,108)$. KYN has been found to moderately impair the killing ability of natural killer cells, whereas KYN and 3-HAA both exert proapoptotic and suppressive effect on these cells $(105,106,109)$. Additionally, TRP depletion and kynurenine metabolites act synergistically to downregulate expression of the $\mathrm{T}$ cell receptor $\zeta$-chain on $\mathrm{CD}^{+} \mathrm{T}$ lymphocytes consequently reducing their cytotoxic capabilities (110). Third, the combination of the presence of kynurenine metabolites and TRP depletion increases the number of regulatory $\mathrm{T}$ cells positive for forkhead box $\mathrm{P} 3\left(\mathrm{FOXP}^{+}\right)$via TGF $\beta$ induction and its impact on naive T cells (110). Moreover, downstream TRP catabolites are able to shift dendritic cells to a tolerogenic phenotype independent of the microenvironment
TRP levels, i.e., without functional IDO-1 $(111,112)$. Therefore, IDO-1 competent dendritic cells also contribute to KP-mediated immune-suppression by contributing to a tolerogenic environment. Together, this plays a substantial role in the development of immune tolerance and the induction of a negative feedback loop that regulates the immune response (113).

\section{$\mathrm{KP}$ and Immune Modulation in the Pathomechanism of MS}

The importance of IDO-1 in immune modulation particularly to counteract autoimmunity has been illustrated in the EAE mouse model. Autoreactive $\mathrm{CD}^{+} \mathrm{T}_{\mathrm{H}} 1$ cells and $\mathrm{T}_{\mathrm{H}} 17$ cells mediate the autoimmune characteristics present in CNS inflammation in MS and in the EAE animal model (114). Indeed, there is widespread evidence that shows the potential of IDO-1 activation to reduce autoimmune inflammation in the CNS. Furthermore, pharmacological inhibition or genetic ablation of IDO-1 exacerbates EAE clinical scores, associated with decreased $\mathrm{T}_{\mathrm{Reg}}$ cell responses and increased $\mathrm{T}_{\mathrm{H}} 1$ and $\mathrm{T}_{\mathrm{H}} 17$ responses $(68,115,116)$. Conversely, the clinical symptoms of EAE can be ameliorated by administration of 3-HAA or its synthetic derivative, Tranilast, and are likely associated with an enhanced expression of TGF $\beta$ by 3 -HAA. This possibly leads to an increase in the number of $T_{\text {Reg }}$ cells, which can suppress the responses of autoreactive $\mathrm{T}$ cells, including the $\mathrm{T}_{\mathrm{H}} 17$ response (115). Furthermore, Xiao et al. reported that dendritic cells pretreated with IFN- $\gamma$ alleviated the histopathological and clinical characteristics of EAE (117). It is hypothesized that IDO- 1 activation is a self-limiting mechanism as both IDO-1 and $\mathrm{KMO}$ are induced by the autoreactive, IFN- $\gamma$ secreting $\mathrm{T}_{\mathrm{H}} 1$ cells (37). This response could be designed to counteract the detrimental effects to the pathological elevation of kynurenine metabolites. This is supported by the observation of toxic levels of QUIN and 3-HK that are reached in the spinal cord but not the brain in EAE animals with concomitant increased activity and expression of $\operatorname{KMO}(67,117,118)$.

The neuroinflammatory process can be significantly decreased by inhibition of IDO-1 enzyme activity, considerably reducing disease exacerbation (119). Monaco et al. revealed corresponding clinical evidence of depressed TRP levels in both serum and CSF of MS patients (69). A subsequent study has yielded conflicting results to these findings, however, a negative correlation between neopterin, a marker of macrophage activity, and TRP levels in the CSF was observed, reflecting IFN- $\boldsymbol{\gamma}$-induced macrophage induction and IDO-1 activation, respectively (120). Although an additional clinical study this year found an increase in IDO-1 activation via QUIN/KYN ratios in MS patients compared with control, there were no significant differences in kynurenine metabolite levels between MS and other neuroinflammatory disorders. However, some patterns emerged upon stratification of disease into acute and chronic phases of disease course such as a high QUIN/KYN ratio in RRMS patients who are in relapse compared with the remission phase. This suggests a high degree of variation in the course of neuroinflammation in MS (74). Initial IDO- 1 activation is thought to be beneficial in MS; however, there is an emergence of a double-edged sword that is present following prolonged exposure. During CNS inflammation, 
increased IDO-1 activity generates kynurenine metabolites that are neuroactive, specifically neurotoxic QUIN (121). Therefore, although IDO-1 activation acts as an anti-inflammatory, its induction could contribute to the neurodegenerative features in MS in the long-term.

\section{Peripheral Blood Monocytes as a Potent Source of Inflammatory KP Metabolites in MS}

Current evidence suggests that excessive activation of the KP in mononuclear phagocytes can participate in the pathogenesis of MS. Activated monocytes and monocyte-derived macrophages (MDMs) are abundantly present in the demyelinating plaques of MS patients and their migration from the periphery to the CNS is necessary for the development of the MS mouse model, EAE. Mononuclear phagocytes, in particular circulating monocytes and MDMs, display high levels of pivotal KP enzymes and can up regulate their expression in response to inflammation (23). Monocytes display considerable plasticity, being found in various tissues and organs as resident macrophages, and patrolling forms with decreased expression of classical inflammation molecules (e.g., Ly6C in mice and CD14/16 in humans). Patrolling forms carry out immune surveillance of the endothelium and rarely extravasate into tissues without immune stimulus or tissue damage (122), in which case they are then alternatively regulated in classical inflammatory form [reviewed in Ref. (123)]. This makes them a potent source of neurotoxic KP metabolites (e.g., QUIN and 3-hydroxykynurenine) to contribute to MS pathology upon their migration and entry to the brain. Indeed, IFN- $\gamma$ also caused upregulation of KMO and QPRT, two enzymes involved in the production of neurotoxic metabolites such as $3-\mathrm{HK}$ and QUIN (12, 23).

Studies of the EAE model have reported a distinct rise in the level of Ly6 $\mathrm{C}^{\text {high }}$ proinflammatory monocytes within the blood stream before onset of clinical signs. At disease onset, or during relapses, proinflammatory monocytes migrate to the CNS, where their numbers directly correlate with the severity of EAE symptoms (124-126) and in turn, with elevated levels of neurotoxic QUIN in the spinal cord (67). This migration might be facilitated by a damaged $\mathrm{BBB}$, one of the earliest clinical findings in MS, thought to play a fundamental role in the development of the disease [see BBB Breakdown and Entry of Immune Cells as a Key Hallmark of Early MS Pathology; Ref. $(86,87)]$. To confirm that monocyte migration to the CNS is involved in EAE pathogenesis, Mishra and colleagues have demonstrated that its inhibition by the candidate MS drug Laquinimod prevented the onset of EAE and its clinical signs (126). More recently, depletion of phagocytotic monocytes by clodronate treatment reduced the severity of EAE symptoms and protected against further axonal loss (127).

In humans, early postmortem studies of MS plaques have correlated the number of macrophages present in chronic demyelinating lesions to the severity of axonal damage responsible for the symptoms of the disease $(128,129)$. Indeed, monocytes have been found in acute demyelinating lesions and in the demyelinating edges of chronic lesions (130). Moreover, advanced microscopy, through $3 \mathrm{D}$ reconstruction of serial block-face scanning electron microscopy images, has been used in a pivotal study of EAE mice where Yamasaki and colleagues demonstrated that MDMs are directly in contact with the axoglial unit and begin demyelination, as opposed to microglia-derived macrophages that were only found adjacent to the lesions and participated in debris clearance (131).

The importance of this study and of the evidence linking activated mononuclear phagocytes to MS is highlighted by different lines of evidence about the KP in these cells: first, the activated MDMs produce 19 times more QUIN than activated microglia (23); second, the CNS-resident cells do not possess the full enzymatic machinery of the KP and are unable to synthesize high quantities of QUIN, but can still produce KYN $(14,22,62)$; third, extra KYN released by astrocytes in the brain can be used by monocytes and MDMs that traffic into the brain to synthesize more QUIN (22); fourth, the level of QUIN produced by monocytes and MDMs is toxic to neurons and to oligodendrocytes $(20,132)$. Overall, this evidence suggests that monocytes and macrophages migrated to the CNS and activated by IFN- $\gamma(133)$ can act as a major reservoir for the secretion and accumulation of damaging QUIN in the brain and spinal cord of MS patients. Therefore, this lends support to the possibility that monocytes and MDMs participate in the pathogenesis of MS via dysregulation of the KP and excessive accumulation of QUIN and associated neurotoxicity of oligodendrocytes and neurons (summarized in Figure 3).

\section{MECHANISMS OF TOXICITY OF KP METABOLITES}

Several decades ago, it was suggested that the kynurenines could act as important endogenous modulators and that they may be involved in the pathogenesis of not only MS but also a number of neurodegenerative diseases such as Alzheimer's, psychological disorders such as schizophrenia and depression, and neuroinflammatory diseases such as HIV-associated neurological disorder (HAND) (57, 59-61, 134-137). Three kynurenines in particular, QUIN, 3-HK, and 3-HAA, were noted for their ability to cause in vitro and in vivo neuronal

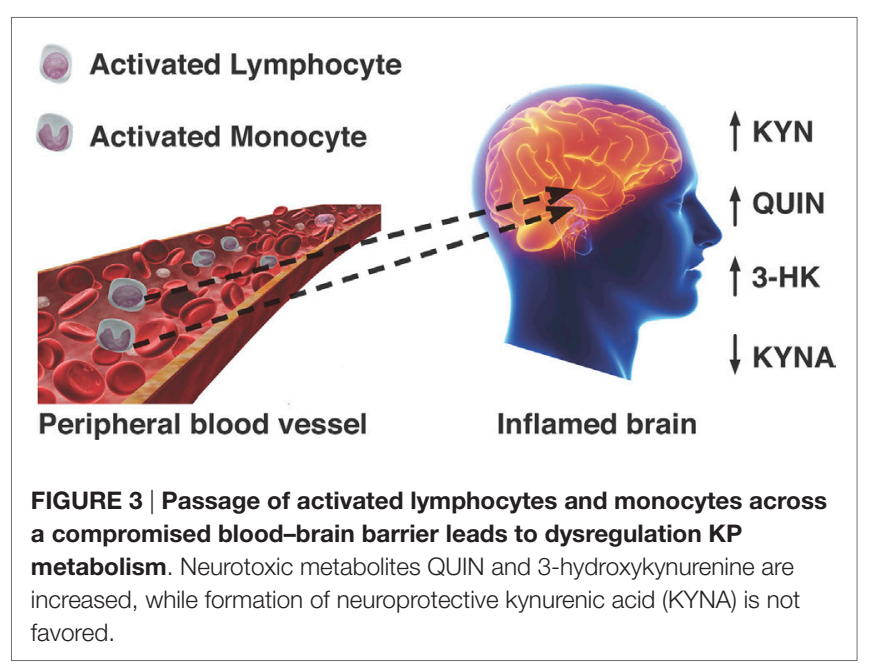


death at slightly elevated concentrations. At low concentrations $(\approx 50 \mathrm{~nm})$, QUIN serves as a substrate for $\mathrm{NAD}^{+}$production in neurons and astrocytes (132).

In pathological conditions, the concentrations of QUIN and 3 -HK found in the CNS are significantly lower than the levels required to perturb neuronal survival (102). Rather, it is considered that these molecules become markedly potent neurotoxins during chronic exposure to low levels and those different populations of neurons are selectively affected by each agent (60). Chiarugi et al. demonstrated that in murine mixed cortical cells, prolonged exposure to QUIN and 3-HK from 24 to $72 \mathrm{~h}$, significantly decreased neurotoxic thresholds from 100 to $1 \mu \mathrm{M}$, respectively, and that exposure to a combination of the two compounds also increased the neurotoxic effects (138). This is of relevance to pathological conditions where both QUIN and 3-HK are simultaneously released and concomitantly accumulate to levels that are neurotoxic leading to chronic exposure of CNS cells (7). QUIN neurotoxicity is primarily attributed to activation of the NMDA receptor and free radical production, and therefore shows complex patterns of neurodegeneration, while 3-HK and 3-HAA are accepted to have a primary role as pro-oxidant metabolites (Table 1) (138).

\section{Quinolinic Acid}

Under normal conditions, QUIN is present in nanomolar concentrations in the brain and is catabolized for the synthesis of $\mathrm{NAD}^{+}$. At low nanomolar physiological concentrations, QUIN is not toxic to neural cells; however, at elevated levels of QUIN (300 nM and possibly even as low as $100 \mathrm{nM}$ with chronic exposure) that are found in inflammatory microenvironments, QUIN begins to be toxic (139). The pathological mechanisms of QUIN neurotoxicity have therefore been found in numerous neurodegenerative processes associated with neuroinflammation such as MS.

Two major factors that render QUIN a potent neurotoxin is the saturation limit of QPRT, and QUINs ability to act as an endogenous weak agonist on the NMDA glutamate receptor. The 3-HAO enzyme, which produces QUIN, has an 80-fold higher reaction velocity than QPRT, the enzyme which degrades QUIN (140). Furthermore, neuronal QPRT is saturated at QUIN concentrations that exceed $500 \mathrm{nM}$ (141). This leads to the production of QUIN at a faster rate than its conversion to $\mathrm{NAD}^{+}$, causing the accumulation of toxic QUIN- and NMDAmediated excitotoxicity (142). Furthermore, surrounding cells (astrocytes, neurons, and microglia) can take up excess released

TABLE 1 | The mechanisms of toxicity following pathological increases in neurotoxic KP metabolites.

\begin{tabular}{lll}
\hline Metabolite & Mechanism & Pathology \\
\hline 3-HK & ROS formation & $\begin{array}{l}\text { Oxidative stress, apoptosis, } \\
\text { potentiation of excitotoxicity }\end{array}$ \\
QUIN & $\begin{array}{l}\text { Generation of free } \\
\text { radicals, NMDA } \\
\text { receptor activation }\end{array}$ & $\begin{array}{l}\text { Excitotoxicity, free radical formation, } \\
\text { mitochondrial dysfunction, apoptosis } \\
\text { or necrosis, cytoskeletal destabilization }\end{array}$ \\
3-HAA & $\begin{array}{l}\text { Generation of free } \\
\text { radicals }\end{array}$ & Oxidative stress, apoptosis \\
&
\end{tabular}

QUIN from the microenvironment, further promoting cellular damage. Moreover, astrocytes favor KYNA synthesis as they do not express KMO (25), whereas microglia preferentially form metabolites of the QUIN branch due to their low expression of KAT (102). Thus, astrocytes appear to maximize the synthesis of KYNA and alone are neuroprotective. However, it should be mentioned that it takes a threefold higher concentration of KA to antagonize the same amount of QUIN (143).

The low levels of QUIN in astrocytes are rapidly degraded. Indeed, the expression of QPRT by astrocytes is IFN- $\gamma$ inducible and explains the rapid catabolism of QUIN in these cells (22). However, the presence of microglia or infiltrating macrophages, such as in MS, means that the high levels of KYN produced by astrocytes can be metabolized to QUIN by neighboring cells (Figure 4) (144). Low levels of neuroprotective PIC may also be synthesized in astrocytes, but production is severely compromised by IFN- $\gamma$ stimulation (25).

\section{NMDA and Excitotoxicity}

Excitotoxicity is a pathological process that results in neuronal damage and death caused by the overactivation of excitatory amino acid receptors. Excitatory amino acids are the primary excitatory neurotransmitters in the hippocampus and cerebral cortex and thus play crucial roles in the psychological functions of neurons. Neuronal excitotoxicity typically refers to the excessive exposure to glutamate, the major excitatory neurotransmitter in the CNS of mammals (145). QUIN is a selective agonist of NMDA receptors, specifically receptor subtypes are composed of NR2A and NR2B subunits. Therefore, as the hippocampus and striatum contain the widest distribution of NMDA receptors, they are areas of the brain most susceptible to QUIN neurotoxicity. Interestingly, neural stem cells (NSCs) in the adult human brain are localized to the subventricular zone of the striatum and the subgranular zone of the hippocampus (146). Considering the importance of adult NSCs in migration and maturation into oligodendrocytes following demyelination in MS, their location in the regions of the brain that exhibit the highest levels of QUIN neurotoxicity, suggests a relationship between KP overactivation and the inhibition of remyelination in MS. In support of this concept, Croitoru-Lamoury et al. found that IFN- $\gamma$ stimulation and concomitant KP activation in mesenchymal stem cells (MSCs) diminished their proliferation and altered their capacity to differentiate. Similar to MSCs, NSCs also express the complete and functional KP enzyme machinery (147). In addition, there is a plethora of studies that have demonstrated that QUIN, as well as KYN and 3-HA, have significant effects on proliferation and activation in specific T cell subsets $(105,107,148)$. These studies indicate a key role of the KP in controlling proliferation and differentiation in various cell types. If pathophysiological concentrations of QUIN do affect NSC proliferation and differentiation, it could provide a relevant mechanism by which remyelination is hindered in MS. Due to the rapid saturation of QPRT, the uptake of QUIN at the synaptic cleft can be delayed, causing further stimulation of the NMDA and continual damage (149). Prolonged activation of the NMDA receptors impairs calcium homeostasis, generates free radicals through the activation of nitric oxide synthesis, and leads to mitochondrial damage and 


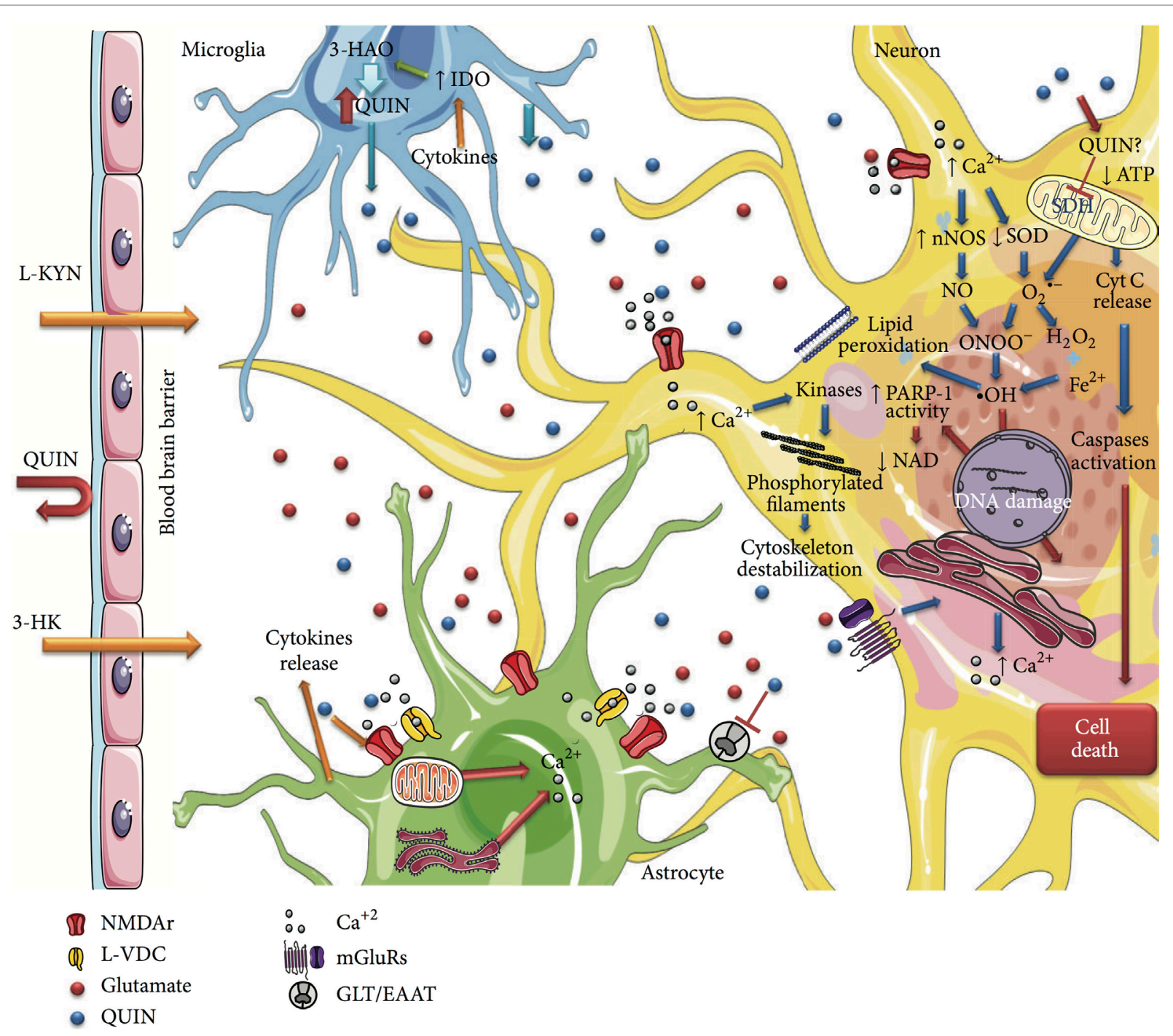

FIGURE 4 | Schematic summarizing the cytotoxicity mechanisms of QUIN in neural cells. This figure is taken from Ref. (149), under Creative Commons license. The significant effects of QUIN on oligodendrocytes have been summarized separately (see Oligodendrocytes Express Only a Subset of KP Enzymes and Oligodendrocytes Are Particularly Sensitive to Quinolinic Acid Toxicity).

initiates programed cell death (150). This induced death has been observed at pathological concentrations in vitro with rat oligodendrocytes $(1 \mathrm{mM})$, primary human neurons and astrocytes $(150 \mathrm{nM})$, and recently in motor neurons $(100 \mathrm{nM})(132,136$, $151,152)$. There is additional evidence showing that QUIN can induce NOS activity in both neurons and astrocytes leading to increases in both poly(ADP-ribose) polymerase (PARP) activity, extracellular lactate dehydrogenase (LDH) activity, and oxidative stress through an increase in production of free radicals (149).

Quinolinic acid toxicity is also due to its direct effect upon the glutamatergic system, which potentiates its primary mechanism of excitotoxicity. In hippocampal slices and cultured astrocytes, QUIN has been shown to increase glutamate release in the synapses, inhibit its re-uptake, and reduce glutamate to glutamine recycling by inhibiting glutamine synthetase activity.
This elevates synaptic glutamate concentration and potentiates excitotoxicity by further overstimulating the NMDA receptor (153-157). Therefore, chronic QUIN exposure is similar to the pathological effects seen in the neurodegeneration of MS. This is in agreement with the findings of Flanagan et al., who found a causal relationship between the degree of clinical severity in the EAE model and the levels of QUIN in the spinal cord (67).

\section{Free Radical Production}

$\mathrm{N}$-methyl-D-aspartic acid receptor activation does not account for all of the neurotoxic effects mediated by QUIN. Indeed, oxidative stress and the generation of free radicals can occur through NMDA receptor-dependent activation or independently by the formation of QUIN-iron complexes. It has been shown that the QUIN-Fe ${ }^{2+}$ complex mediates the formation of reactive 
oxygen species (ROS) through the Fenton reaction (Figure 5) leading to downstream lipid peroxidation and in vitro DNA damage (158).

This effect was attenuated when an alternative ligand for iron, the nucleoside analog acyclovir, was added. The removal of iron from QUIN inhibited lipid peroxidation and decreased the production of superoxide anion radicals demonstrating that the ligand identity is important to ROS development (159). Supporting this hypothesis, QUIN has also been shown to dysregulate redox homeostasis by affecting the endogenous antioxidants such as reduced glutathione, and depleting enzymes that scavenge free radicals, such as copper/zinc-superoxide dismutase (CuZn-SOD). QUIN has been shown to modify the activities of several endogenous antioxidants and deplete the activity of cytosolic CuZn-SOD, therefore exerting stress on primary antioxidant defense mechanisms (160). Moreover, these pro-oxidant effects of QUIN can be prevented by treatment with different antioxidants such as melatonin and pyruvate. The pro-oxidant toxicity of QUIN is likely to occur by decreasing the nuclear translocation of the transcription factor NF-E2related factor 2 (Nrf2), and important transcriptional activator of antioxidant response element (ARE). The Nrf2/ARE pathway induces phase II antioxidant enzymes and is therefore an important promoter to detoxify oxidants. Tert-butylhydroquinone (tBHQ) exhibits antioxidant properties through its ability to induce Nrf2 nuclear translocation, thus activating ARE. In rat striatal slices, QUIN was observed to decrease nuclear Nrf2, while tBHQ protected against QUIN-induced mitochondrial dysfunction and lipid peroxidation, and partially recovered glutathione-S-transferase activity (161). This suggests that QUIN toxicity is also associated with a silencing of phase II antioxidant enzymes, thereby generating oxidative stress and simultaneously reducing antioxidant defenses.

In recent years, evidence implicates the roles of nitrative and oxidative damage and mitochondrial dysfunction in directly causing acute axonal damage in new inflammatory lesions in MS that may lead to degeneration $(4,162)$. An in vivo study using confocal microscopy of early lesions in the EAE found free radicals caused early mitochondrial damage at inflammation sites prior to demyelination (163). Of interest, scavengers of reactive oxygen and nitrogen species could reverse this injury, indicating a potential neuroprotective strategy. It is thought that oxidative and nitrative damage advances both the initial and chronic active lesion in MS. Indeed, Haider et al. found that in active MS plaques and not in control brain tissue, there were high levels of oxidized lipids and DNA. DNA oxidation occurred mainly in oligodendrocyte nuclei, which also exhibited signs of apoptosis. Additionally, DNA and lipid oxidation correlated significantly with inflammation, determined by quantifying human leukocyte antigen-D expressing macrophages and microglia and

\section{$\mathrm{Fe}^{2+}+\mathrm{H}_{2} \mathrm{O}_{2} \rightarrow \mathrm{Fe}^{3+}+\mathrm{OH}^{-}+\mathrm{OH} \cdot$}

FIGURE 5 | The Fenton reaction for the production of reactive oxygen species.
$\mathrm{CD}^{+} \mathrm{T}$ cells in the lesions (164). Given the key role of oxidative damage in driving MS pathology, and QUIN-mediated free radical production, this could constitute an additional means by which the KP contributes to MS.

However, more recent reports have suggested a dual role of QUIN in being able to both scavenge and produce ROS, depending on the chemical environment and its concentration (165). Other small molecules that act as antioxidants, such as specific vitamins and metabolites, also typically show this double behavior and likely play a relevant role in maintaining redox homeostasis and oxidative balance. In support of this, studies have indicated that lower concentrations of QUIN affect the redox homeostasis of iron maintaining the $\mathrm{Fe}(\mathrm{II}) / \mathrm{Fe}$ (III) equilibrium (166). Indeed, it has been suggested that at low QUIN concentrations, QUIN participates as an antioxidant and that the combination of high levels of ROS and QUIN are required for oxidative stress and cytotoxicity (166). It should be noted that these experiments examined QUIN activity in non-cellular based assays. The complex chemistry of QUIN will be largely milieu dependent and based on specific cellular environments.

\section{Cytoskeleton}

Quinolinic acid has also been shown to induce damage to dendrites and axons with recent evidence showing that toxic QUIN levels phosphorylate structural proteins, thereby destabilizing the cytoskeleton $(167,168)$. The cytoskeleton is vital for neuronal cell shape and function and is involved in maintaining synapse formation, internal transport of molecules, and neurite outgrowth. Furthermore, it has been well established that axonal injury, including axonal transport disruption, is prevalent in active MS lesions (169). Acute intrastriatal administration of QUIN was found to cause NMDA-mediated $\mathrm{Ca}^{2+}$ influx and oxidative stress that resulted in the hyperphosphorylation of intermediate filaments in neural striatal cells $(149,168)$. In rat striatal slices, $100 \mu \mathrm{M}$ QUIN altered the cytoskeletal homeostasis of both astrocytes and neurons. In astrocytes, QUIN's actions were mediated by a rise in $\mathrm{Ca}^{2+}$ influx through L-type voltage-dependent $\mathrm{Ca}^{2+}$ channels (L-VDCC) and NMDA receptors, whereas in neurons, additional actions involved intracellular $\mathrm{Ca}^{2+}$ and metabotropic glutamate receptors. Both cases similarly result in a cascade of second messenger-dependent kinase activation, the phosphorylation of domain sites on neurofilament subunits and GFAP and irregular assembly of intermediate filaments in both glia and neuronal cells $(167,170,171)$.

Additionally, work performed by Rahman et al. demonstrated that prolonged exposure to QUIN-induced significant changes to the structure of human neurons including decreasing organelles, dendritic beading, and the disruption of microtubules. The observed structural perturbations were associated with a decrease in major tau phosphatases expression and activity and consequently a concomitant increase in tau phosphorylation at multiple sites (168). In line with this evidence, Anderson et al. identified irregular tau phosphorylation in the EAE model and in progressive MS patients (169). Abnormal tau phosphorylation and insoluble tau accumulation is associated with both axonal and neuronal loss, which parallels the transition of relapseremitting to the chronic, secondary progressive stage in EAE. 
Analysis of secondary progressive brain tissue in humans with MS revealed significant abnormal phosphorylated tau and insoluble tau formation. This observation was focused on areas dominated by demyelination, gliosis, and neuronal injury. Given that QUIN has direct effects on gliosis, neuron survival, and tau phosphorylation, this further supports a role for QUIN in the neurodegeneration associated with MS. Interestingly, QUIN may also have an effect on intracellular $\mathrm{Ca}^{2+}$ signaling as QUINinduced NMDA receptor overstimulation causes early damage to the sarco/endoplasmic reticulum $\mathrm{Ca}^{2+}$-ATPase (SERCA) pump, thereby disturbing intracellular $\mathrm{Ca}^{2+}$ regulations (172).

\section{3-Hydroxykynurenine}

Similar to QUIN, the plasma, brain, and spinal cord levels of 3-HK are elevated in EAE rats (118). There is evidence that $3-\mathrm{HK}$ is a neurotoxic metabolite and it, therefore, may have an important role in the neurodegeneration of MS (173). To date, there is a multitude of literature providing evidence that $3-\mathrm{HK}$ is a pro-oxidant and a potent generator of reactive species that induces apoptosis. This characterization has been performed in vitro at concentrations that are supraphysiological, ranging from $10 \mu \mathrm{M}$ to $1 \mathrm{mM}$. These concentrations are considerably higher than both normal brain $(\sim 0.08-0.3 \mu \mathrm{M})$ and pathological brain $(0.3-1.2 \mu \mathrm{M})$ concentrations and could evoke toxicity not observed under normal conditions (113). In support of this, there is growing evidence suggesting that $3-\mathrm{HK}$ is an endogenous antioxidant. Despite these considerations, $3-\mathrm{HK}$ is described as neurotoxic and the dual effects of this metabolite will be discussed below.

In the presence of oxygen and at neutral $\mathrm{pH}, 3-\mathrm{HK}$ easily undergoes auto-oxidation forming $o$-semiaminoquinone. The oxidation of 3 -HK has been found to (1) generate ROS, which promotes lipid oxidation, protein modification, inflammatory response modulation, and DNA damage; (2) reduce trace transition metals, including $\mathrm{Fe}^{3+}$ and $\mathrm{Cu}^{2+}$, to pro-oxidants, $\mathrm{Fe}^{2+}$ and $\mathrm{Cu}^{+}$, capable of generating further radical formation in Fenton-like reactions; (3) $o$-semiaminoquinone readily reacts with oxygen generating quinone-imine, another highly reactive product which also participates in additional oxidative reactions (113). In line with this, 3-HK and 3-HAA $(10 \mu \mathrm{M})$ induced neuronal cell death with apoptotic features following generation of ROS in primary striatal neurons. This toxicity was dependent on its cellular uptake by large neutral amino acid transporters in a sodium-dependent process and the increase in intracellular ROS, as various antioxidants inhibited this process $(174,175)$. Cell death occurred via p38 death signaling and was independent of caspase-3 mechanisms. However, cerebellar granule neurons appeared more resistant than striatal cells to HK-induced damage, suggesting toxicity specificity $(250 \mu \mathrm{M})(176,177)$.

Interestingly, when human astrocytes are treated with $3-\mathrm{HK}$ at concentrations lower than $100 \mathrm{nM}$, intracellular $\mathrm{NAD}^{+}$levels are significantly augmented. At doses above $100 \mathrm{nM}$, however, NAD ${ }^{+}$ levels are significantly decreased and extracellular LDH activity is increased (178). $\mathrm{NAD}^{+}$is a molecule involved in many metabolic processes and is a vital cofactor for several enzymes. For example, $\mathrm{NAD}^{+}$is a precursor for agents that mobilize calcium and regulate gene transcription through chromatin-associated protein modification and is a substrate for ADP ribosylation of proteins
$(179,180)$. Therefore, alterations in 3 -HK concentrations may indirectly change (1) gene expression, (2) DNA repair, and (3) intracellular $\mathrm{Ca}^{2+}$ levels.

There have been few studies of the in vivo effect of 3-HK. Of these, the most notable examined the synergistic possibility of the combination of 3-HK and QUIN in neurotoxicity. In rat brains, intrastriatial injection of 3-HK (5 nM) or QUIN (15 nM) individually caused no or marginal damage, whereas coinjection of the two metabolites caused impaired rotational behavior and significant increases in the volume of lesions (113). Notably, there was an absence in de novo generation of QUIN suggesting that the in vivo conversion of QUIN from 3-HK was not the mechanism behind the potentiation of QUIN toxicity (181). This could be a result of coinjection, as there is a possibility of chemical interactions that could modify the reactive components of each reagent (113). These findings might also indicate that the normal brains capacity to scavenge radicals is sufficient to counteract 3-HK-induced radical formation and prevent cellular apoptosis (182).

\section{3-HAA}

At present, there have been several studies that have examined 3-HAA activity in conjunction with 3-HK. It has been recognized that 3-HAA exhibits similar characteristics to 3-HK to generate superoxide anions by undergoing auto-oxidation and, thus, initiate apoptosis (183). Recent evidence has also indicated that 3-HAA has immunomodulatory roles that could play important roles in MS (184). This emerging evidence is discussed in Section "KP Influence on the Immune System" above.

\section{OLIGODENDROCYTES AS A TARGET CELL FOR ELEVATED LEVELS OF KP METABOLITES IN MS}

Oligodendrocytes are demyelinated and perish in MS most probably through the action of autoimmune $\mathrm{T}$ cells and associated neuroinflammation. As there is substantial influx of immune cells into the brain in MS, a significant body of evidence exists supporting a dysregulated KP in MS, which can favor the production of neurotoxic metabolites which further compromise oligodendrocyte health and function and could also contribute to neuronal atrophy. The known studies investigating the presence of KP metabolites and/or KP action on oligodendrocytes in MS are discussed below.

\section{Oligodendrocytes Express Only a Subset of KP Enzymes}

Overall, while oligodendrocytes express several KP enzymes and may be able to uptake certain KP metabolites and participate in their metabolism, they appear not to express IDO-1, meaning that they are incapable of modulating $T$ cell phenotype and KP action by metabolizing TRP. Radiolabeled QUIN was not detected in rat brain cultured oligodendrocytes after IFN- $\gamma$ stimulation and incubation with radiolabeled TRP (185), leading to the subsequent conclusion following the study of human 
primary oligodendrocytes that oligodendrocytes are not capable of synthesizing QUIN de novo, rather they metabolize it (62). Via PCR, we demonstrated that IDO-1 is not expressed in human primary oligodendrocytes, even with IFN- $\gamma$ stimulation (62), though data on adult oligodendrocytes are currently lacking. The enzymes tryptophan-2,3-dioxygenase (TDO) and kynurenine amino transferases II (KATII) were also not expressed, while KAT-I and enzymes further down the KP [KYNU, KMO, 3-hydroxyanthranilate oxygenase (3-HAO), and QPRT] were expressed, and increased in expression with IFN- $\gamma$ stimulation.

The oligodendrocytes constitutively express the QPRT enzyme shows that they have the enzymatic machinery for further metabolism of QUIN to the essential cofactor $\mathrm{NAD}^{+}(62)$. QPRT was only modestly increased in expression in human oligodendrocytes in response to IFN- $\gamma$ stimulation (62), suggesting that in inflammatory environments it could be easily saturated (considered further in Section "NMDA and Excitotoxicity"). Clearly, with regard to oligodendrocytes, the expression of QPRT and function in disease requires further study. Oligodendrocytes can also produce the neuroprotective PIC at concentrations of 45-55 nM [depending on whether IFN- $\gamma$ stimulus is present (62)]; intriguingly, this was present at substantial $(\approx 10$-fold) excess compared with QUIN (even with IFN- $\gamma$ stimulus) suggesting the predominant balance of the metabolism of the common QUIN/ PIC precursor $\alpha$-amino- $\beta$-carboxymuconate- $\varepsilon$-semialdehyde (ACMS) is skewed toward PIC production, or alternatively, that excess QUIN in oligodendrocytes is rapidly converted to NAD ${ }^{+}$ via QPRT. Our recent experiments in cultured oligodendrocytes [Ref. (20) and discussed in Section "Oligodendrocytes Are Particularly Sensitive to Quinolinic Acid Toxicity] currently do not support the latter hypothesis. The presence and function of 2-amino-3-carboxymuconate-6-semialdehyde decarboxylase (ACMSD) in oligodendrocytes is unknown at present, although from other studies its enzymatic activity is known to be partially inhibited by KYNA, QUIN, and PIC (186).

\section{Oligodendrocytes Are Particularly Sensitive to Quinolinic Acid Toxicity}

Quinolinic acid is an NMDA receptor agonist and excitotoxin $(139,187)$, and oligodendrocytes are susceptible to NMDARmediated excitotoxicity with subsequent alterations to $\mathrm{Ca}^{2+}$ and other intracellular signaling that culminates in apoptosis (151, 152, 188-190). Therefore, in the context of activated monocyte influx during inflammatory demyelination episodes and secretion of QUIN, excitotoxicity induced by QUIN on oligodendrocytes is a major consideration in the progression of MS pathology. Conventional MS treatments can reduce the frequency of inflammatory episodes but do little to counteract the demyelination and death of oligodendrocytes (191).

Activated monocytes can enter the brain during BBB breakdown events and secrete QUIN or activate KP in neural cells by secretion of IFN- $\gamma$. Exposure of oligodendrocytes to excitotoxic QUIN at high levels, therefore, constitutes a further insult in concert with autoimmune-mediated demyelination. Indeed, we recently demonstrated by microscopy that QUIN can be rapidly taken up in vitro by cultured oligodendrocyte cell lines (substantial uptake was observed within 30-90 min), without significant degradation of QUIN. One possibility for the enhanced uptake is that the QPRT enzyme responsible for further metabolism of QUIN is saturated at a lower concentration, and hence in inflammatory circumstances QUIN accumulates in oligodendrocytes similar to that observed in neurons that are associated with Alzheimer's pathology (168).

More importantly to MS pathology, these cells were also very sensitive to QUIN toxicity, which induced apoptotic cell death (LD50 $0.5-1.0 \mu \mathrm{M}$ in oligodendrocyte cell lines) (20). This is supported by the findings of others $(151,152)$. Apoptosis could be completely reversed by treatment with a monoclonal blocking antibody recognizing QUIN or by the use of specific IDO-1 enzyme inhibitors (to abolish QUIN production in monocytelineage cells) (20). In addition to QUIN concentration, chronicity of exposure and cell type is likely additional determinants of excitotoxic effects on brain cells. In the context of an inflammatory microenvironment, T-cell-induced damage to the myelin sheath as well as the toxicity induced by the presence of elevated levels of monocyte-produced QUIN are likely to significantly contribute to apoptosis of oligodendrocytes observed in MS.

Oligodendrocytes express multiple types of glutamate receptors, including $N$-methyl-D-aspartate (NMDA), alphaamino-3-hydroxy-5-methylisoxazole-4-propionic acid (AMPA), and kainate. In white matter ischemic damage, excitotoxic cell death of oligodendrocytes via AMPA and kainate receptors are most often implicated (190, 192, 193). Oligodendrocytes lacking glutamate receptor subunit 2 (GluR2) are susceptible to AMPA/Kainate-induced excitotoxicity $(190,194)$. Spinal gray (195) and the myelinating processes of white matter oligodendrocytes express some NMDA receptors $(188,189,196)$. Subsequent work confirmed that oligodendrocytes have an unusual NMDA receptor composition compared with neurons or other cells (197). Oligodendrocytes do express NR2A and 2B subunits (188), which have been confirmed in Xenopus oocytes to bind and be activated by QUIN, while NR2C subunits have 10-fold less affinity (198).

Although there is evidence that blockade of NMDA receptors is neuroprotective in inflammatory CNS diseases, and indeed can improve survival of oligodendrocytes $(199,200)$, future therapeutic use of this strategy needs to be tempered with an understanding that a "one-size-fits-all" approach such as broadly targeting NMDA receptors may not result in complete neurological disease resolution, and indeed, could have substantial side-effects including effects on other cell types such as neurons $(201,202)$. Hence, in our opinion, there is a clear notion that understanding the role of the KP in immune modulation and production of neurotoxic metabolites with action on oligodendrocytes could lead to the generation of alternative kinds of targeted therapies, which may be used as novel treatments for MS and possibly other autoimmune diseases.

\section{MODULATION OF THE KP AS A THERAPEUTIC STRATEGY IN MS}

Many therapeutic strategies are currently being pursued to protect against dysregulated KP metabolism in neurological diseases 
[summarized in Ref. (203)]. In the context of MS, current treatments are anti-inflammatory and although they effectively reduce the number and duration of relapses they do not appear capable of preventing long-term disability and mortality (204). This demonstrates an obvious lack of therapies that target the neurodegenerative components of the disease and that facilitate remyelination. As the KP is highly implicated in the pathophysiology of neurodegeneration and neuroinflammation, KP enzyme inhibitors together with kynurenine metabolites and their pharmacological analogs, could represent promising new therapeutic strategies that could target these mechanisms. A synthetic analog of the TRP metabolite $N$-[3,4-dimethoxycinnamoyl]-anthranilic acid (3,4-DAA), known commercially as Tranilast, has shown promising results in both in vivo and in vitro experiments (Figure 6).

Hertenstein et al. found that 3,4-DAA inhibited $\mathrm{CD}^{+} \mathrm{T}$ cell activation and proliferation and naive $\mathrm{CD}^{+} \mathrm{T}$ cells, although to a lesser extent (205). EAE animals treated with 3-HK or its synthetic derivate, Tranilast, showed reduced symptoms, number of relapses, and fewer inflammatory nodes in the spinal cord and brain $(115,206)$. In the EAE mouse model, the activity of KMO is significantly increased in spinal cords and this correlates with an increase in QUIN and 3-HK to neurotoxic levels. Administration of the KMO inhibitor Ro 61-8048 reduced the rise in levels of both QUIN and 3-HK, increased neuroprotective KYNA production and significantly alleviated disease progression (118). A number of immunomodulatory drugs such as leflunomide (the active metabolite is Teriflunomide) and Laquinimod, are structurally analogous to KYN and KYNA, respectively, and exhibit immunosuppressive properties by promoting a $\mathrm{T}_{\mathrm{H}} 2$ profile through a shift in cytokine balance and inhibiting activated $\mathrm{T}$ cells. These drugs were shown to ameliorate disease in EAE and Teriflunomide passed phase III clinical trials and is currently approved by the FDA. Although Laquinimod demonstrated only modest effects on the relapse rate in RRMS in phase III trials, it showed significant reductions in brain atrophy and is currently in phase III trials for PPMS, in which there are currently no approved disease-modifying treatments $(207,208)$. Together, the immunomodulatory and structural similarities of these drug candidates to kynurenines strongly implicate kynurenine analogs as novel and intriguing therapies for MS. The concept of KP therapies in MS is further supported by in vitro findings that QUIN is toxic to oligodendrocytes. Importantly, inhibiting IDO-1 (1-MT or berberine) or neutralizing QUIN directly with anti-QUIN antibodies overcame the toxicity toward oligodendrocytes that QUIN displayed (20).

Given that QUIN is present at pathophysiological concentrations that correlates with severity in EAE mice, manipulations that directly modulate its concentration-such as inhibition of IDO-1 or KMO are of considerable interest. However, systemic inhibition of IDO-1 has been demonstrated to exacerbate disease in EAE mice (18). This is due to the double nature of IDO-1 activation, where initial activation is immunosuppressive and is an important mechanism to counteract the autoimmune response; however, prolonged activation leads to the production of neurotoxic and oligotoxic kynurenines, thereby contributing to the pathology of MS. It is, therefore, likely that the timing of intervention is important. It is clear through both experimental and indirect evidence that the complex anti-inflammatory and neuroprotective properties of the KP metabolites have a fundamental link with MS. This warrants screening of these candidate drugs and highlights their therapeutic potential in MS.

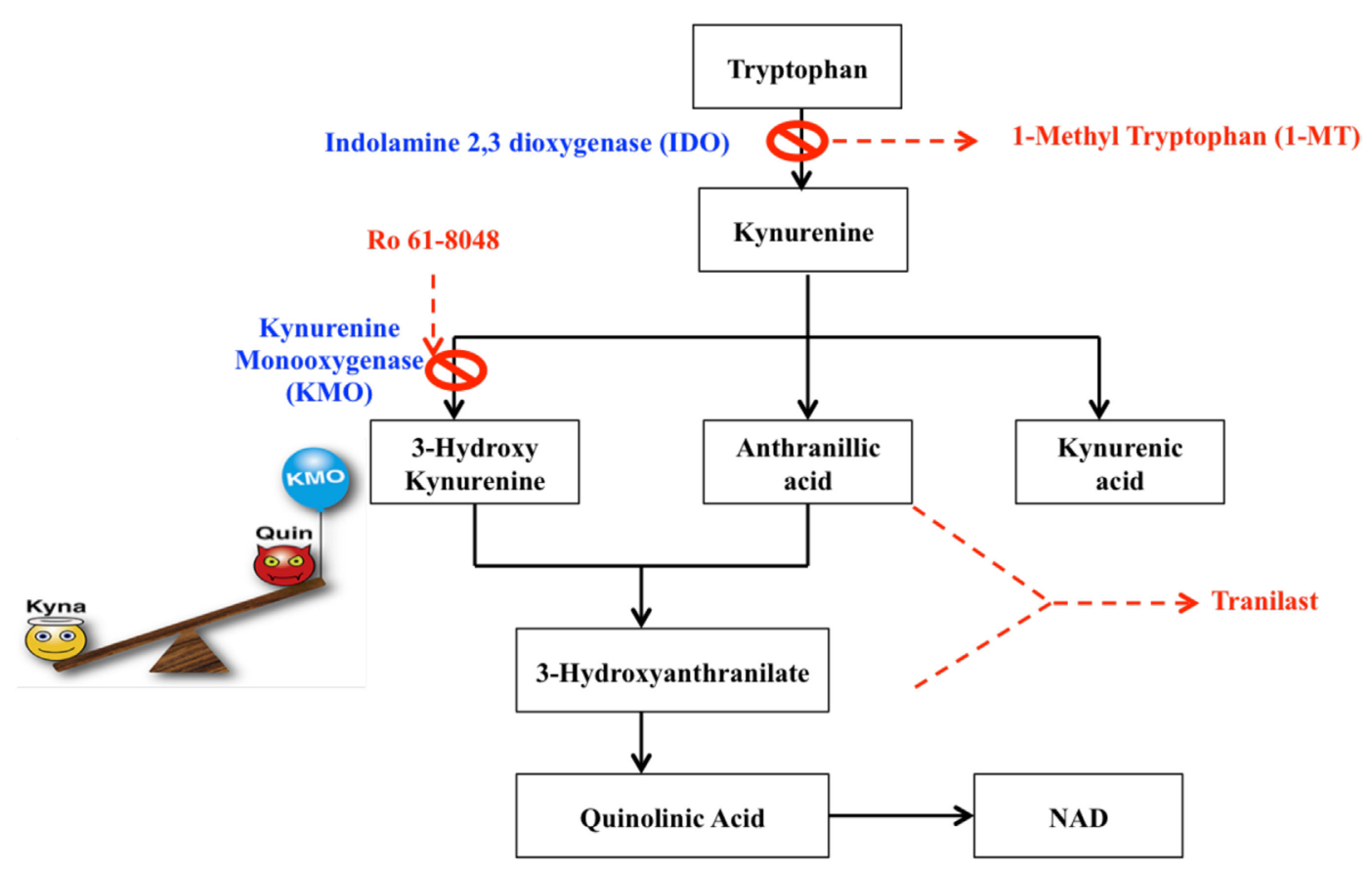

FIGURE 6 | Potential therapeutic targets for the modulation of the kynurenine pathway. Dashed boxes indicate pharmacological analogs, whereas crosses indicate inhibitors. 


\section{MICROSCOPE IMAGING AS A CENTRAL TOOL FOR ADVANCING KNOWLEDGE OF MS PATHOLOGY}

This review has discussed (particularly in Sections "The BBB Performs a Critical Role in Maintaining Barrier Integrity and Permeability, Which Is Lost in MS" and "Peripheral Blood Monocytes as a Potent Source of Inflammatory KP Metabolites in MS") the significant achievements which have been generated by the use of imaging technology in MS studies, particularly noninvasive imaging such as MRI, and also correlative microscopy and histopathology. For mouse models of MS, multiphoton microscopy (Figure 7) has traditionally been the imaging technique of choice for imaging intact thick tissue sections $(>200 \mu \mathrm{m})$ (209-212). Additionally, the easy availability of intravital imaging technology has greatly expanded the opportunity of directly observing the spatio-temporal context in which inflammatory events unfold in situ within the natural microenvironment of the CNS (213-215). Therefore, it is fast becoming the preferred modality for analyzing neuroinflammation in vivo. The advent of highly streamlined surgical protocols used in combination with two-photon intravital microscopy (2P-IVM) has proven to be powerful for characterizing the cellular and molecular mechanisms that underlie neuroinflammation (216-218). Its applicability across a breadth of neurological disciplines have helped

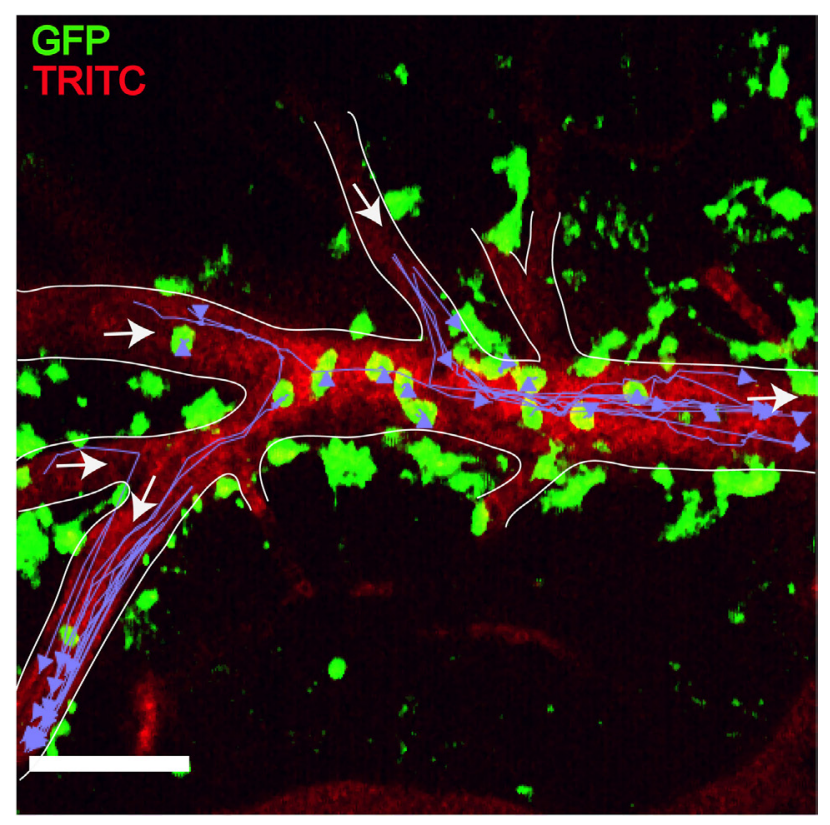

FIGURE 7 | Plasmodium-primed CD8 ${ }^{+}$T cells induce monocyte accumulation in MacGreen $\times$ RAG $^{-1-}$ mice. PbA-infected

MacGreen $\times \mathrm{RAG}^{-/}$mice that had received primed $\mathrm{CD} 8^{+} \mathrm{T}$ cells underwent intravital imaging on day 7 p.i. ( $n=3$ mice/group). Representative snapshot shows severe levels of monocyte accumulation in the blood vessels. Scale bar represents $60 \mu \mathrm{m}$. Migratory paths of monocytes are shown as purple tracks. Blood vessels are marked by infusion of TRITC-conjugated dextran. White arrows indicate direction of blood flow. Data are a mean of two to three independent experiments [figure reproduced from Ref. (215), under Creative Commons Attribution license]. identify novel routes of immune cell entry, their locomotion patterns, intravascular and transendothelial migration, homing, interactions with endothelial, immune, stromal, and neuronal cells, among others (219-223). These studies have significantly increased our understanding of the initiation and perpetuation of inflammation within the CNS and laid an excellent platform for making further advances within the field.

Our knowledge of how immune cells are recruited to the CNS; navigate through the CNS tissue; respond to self-antigens, pathogens, toxic metabolites, etc., and how they contribute to inflammatory diseases has been greatly informed by intravital imaging studies on EAE, one of the most intensely studied animal models of MS (224-228). In the past, EAE studies have been limited by the requirement for sophisticated imaging tools that can track $\mathrm{T}$ cell entry, behavior, and transmigration at cellular and subcellular resolution within the CNS. The complexities of tracking $\mathrm{T}$ cells in the CNS are related to the unique anatomical barriers such as the BBB that partly seclude the CNS from the constantly changing microenvironment of the blood stream, and its circulating immune cells $(78,229,230)$. 2P-IVM (fluorochrome excited by two photons of $976 \mathrm{~nm}$ wavelength) circumvents some of these limitations due to its twin advantages of low phototoxicity and photobleaching, which enable long-term visualization $(\sim 1.5-6 \mathrm{~h})$ and better penetrance $(250-300 \mu \mathrm{m})$ of the CNS tissue (216).

Two-photon intravital microscopy studies have revealed that $\mathrm{T}_{\mathrm{MBP}}$ cells ( $\mathrm{T}$ cells recognizing oligodendrocyte $\mathrm{MBP}$ ) first appear in the CNS vasculature during the build-up to EAE (225). Although it was not possible initially to ascertain the precise location of the infiltrating $\mathrm{T}_{\mathrm{MBP}}$ cells using video microscopy, it is now clear that $\sim 80 \%$ of the infiltrating $\mathrm{T}_{\mathrm{MBP}}$ cells arrest and then crawl along the inner surface of the vessel wall soon after their entry (101). Only $\mathrm{T}_{\mathrm{MBP}}$ cells that carry specific molecular signatures such as VLA-4 transmigrate through the BBB where they spend $80 \%$ of their time crawling once again on the abluminal surface of the vasculature, before detaching and crawling finally on the surface of the neuropil $(224,225)$. Therefore, infiltrating $\mathrm{T}_{\mathrm{MBP}}$ cells crawl along three different cellular planes at different velocities with the end objective of "seeking" and contacting perivascular macrophages (PVM) that reside in the perivascular space $(101,231)$

Real-time imaging studies have shown that after gaining passage into the perivascular space, $\mathrm{T}_{\mathrm{MBP}}$ cells make short or longlasting contact (>10 min) with PVM, antigen-presenting cells that strategically line blood vessels and monitor the environment with thin, motile cellular processes $(209,225,232)$. Polarization of TCR and adhesion molecules such as LFA-1 as well as nuclear translocation of fluorescent NFAT, indicative of calciumdependent $\mathrm{T}$ cell activation have been reported to occur at these contact points $(227,233)$. Interestingly, perivascular microglial and PVM clustering is one of the first pathological alterations that occurs in response to $\mathrm{BBB}$ disruption, during the build-up to EAE (234). As part of this alteration, cell body motility and process extension of microglia and PVM are specifically directed toward the affected vasculature where BBB disruption occurs. Thus, PVM clustering may only serve to enhance the chance of $\mathrm{T}_{\mathrm{MBP}}$ cells encountering cognate antigen and invading the CNS parenchyma, besides its reported contribution to axonal damage. 
Studies have shown that a majority of the $\mathrm{T}_{\mathrm{MBP}}$ cells that invade the CNS parenchyma meander through the tissue (233). A small number exhibited confined motility, reminiscent of cells that have found cognate autoantigen. At first glance, these $\mathrm{T}_{\mathrm{MBP}}$ cells appeared stationary; however, closer examination showed that they were remarkably agitated, rapidly extending and retracting cell protrusions and migrating at very low velocities through the tissue. Expectedly, extended recordings spanning several hours were necessary to resolve these fine locomotion patterns of $\mathrm{T}_{\mathrm{MBP}}$ cells at the single cell level, especially since the sum of their directionality and length of migration remained close to zero $(101,233)$.

Despite the increasing number of real-time multiphoton studies that have yielded tremendous insights into the behavior of T cells in the processes leading to lesion formation at the single cell level in mouse MS models, application of this technology to the study of monocytes has yet to emerge. As described in Section "Peripheral Blood Monocytes as a Potent Source of Inflammatory KP Metabolites in MS," evidence supports a role of activated monocytes in production of neurotoxic metabolites, which could contribute to MS pathology. Therefore, future studies to understand the behavior of monocytes as they pass into the brain parenchyma and interact closely with cells such as oligodendrocytes will provide additional insight to those gained recently by serial block-face reconstruction ultrastructure imaging (131).

\section{SUMMARY AND CONCLUSION}

Imaging is now a central tool in MS research and treatment, allowing clinicians to evaluate the extent of the pathology and effectiveness of treatments while microscopy advances have allowed us to, in the case of multiphoton microscopy, peer deep into the brain to uncover mechanisms involved in immune cell trafficking and BBB dysfunction. Electron microscopy advances

\section{REFERENCES}

1. Watzlawik JO, Wootla B, Rodriguez M. Tryptophan catabolites and their impact on multiple sclerosis progression. Curr Pharm Des (2016) 22:1049-59. doi:10.2174/1381612822666151215095940

2. Dendrou CA, Fugger L, Friese MA. Immunopathology of multiple sclerosis. Nat Rev Immunol (2015) 15:545-58. doi:10.1038/nri3871

3. Chard D, Miller D. Grey matter pathology in clinically early multiple sclerosis: evidence from magnetic resonance imaging. J Neurol Sci (2009) 282:5-11. doi:10.1016/j.jns.2009.01.012

4. Mitchell AJ, Benito-Leon J, Gonzalez JM, Rivera-Navarro J. Quality of life and its assessment in multiple sclerosis: integrating physical and psychological components of wellbeing. Lancet Neurol (2005) 4:556-66. doi:10.1016/ S1474-4422(05)70166-6

5. Bashir K, Whitaker JN. Current immunotherapy for demyelinating diseases. Arch Neurol (2002) 59:726-31. doi:10.1001/archneur.59.5.726

6. Goldenberg MM. Multiple sclerosis review. P T (2012) 37:175-84.

7. Heyes MP, Saito K, Crowley JS, Davis LE, Demitrack MA, Der M, et al. Quinolinic acid and kynurenine pathway metabolism in inflammatory and non-inflammatory neurological disease. Brain (1992) 115(Pt 5):1249-73. doi:10.1093/brain/115.5.1249

8. Sanni LA, Thomas SR, Tattam BN, Moore DE, Chaudhri G, Stocker R, et al. Dramatic changes in oxidative tryptophan metabolism along the kynurenine have also allowed automated processing and imaging of slices of tissue sections, allowing the ultrastructure of cell-cell interactions in MS to be confirmed. While MS is traditionally thought of in the context of aberrant autoimmune triggering of T cells, the multifaceted nature of the disease has emerged through increasing evidence of neurodegenerative pathology and the involvement of other cell types such as monocytes, and in turn signaling systems including the KP. This increasing recognition has led to the current notion of MS as involving a substantial degenerative disorder component (9). In this context, over the past two decades, a substantial body of literature has proven that dysregulation of the KP results in overproduction of neurotoxic metabolites that can potently kill brain cells. In MS, the extent of the pathology and disease progression correlates with elevated levels of neurotoxic metabolites which can contribute to the death of myelinating oligodendrocytes and their neighboring neurons, including during autoimmune inflammatory episodes. Therapeutic interventions to reduce the levels of damaging KP metabolites and improve oligodendrocyte and neuron survival are in continual development and progress toward clinical utility.

\section{AUTHOR CONTRIBUTIONS}

ML, BV, GS, NF, MN, SP, CL, GG, and BB - manuscript writing and final approval of manuscript. ML, BV, GS, SP, and NF preparation of figures.

\section{FUNDING}

The authors acknowledge the generous support and funding of the Peter Duncan Neurosciences Research Unit and a University of New South Wales (UNSW) Goldstar grant for this study. Neither of these agencies influenced the decision to publish.

pathway in experimental cerebral and noncerebral malaria. Am J Pathol (1998) 152:611-9.

9. Stys PK, Zamponi GW, Van Minnen J, Geurts JJ. Will the real multiple sclerosis please stand up? Nat Rev Neurosci (2012) 13:507-14. doi:10.1038/ nrn3275

10. Schwarcz R. The kynurenine pathway of tryptophan degradation as a drug target. Curr Opin Pharmacol (2004) 4:12-7. doi:10.1016/j.coph.2003.10.006

11. Stone TW, Darlington LG. The kynurenine pathway as a therapeutic target in cognitive and neurodegenerative disorders. Br J Pharmacol (2013) 169:1211-27. doi:10.1111/bph.12230

12. Jones SP, Franco NF, Varney B, Sundaram G, Brown DA, De Bie J, et al. Expression of the kynurenine pathway in human peripheral blood mononuclear cells: implications for inflammatory and neurodegenerative disease. PLoS One (2015) 10:e0131389. doi:10.1371/journal.pone.0131389

13. Torok N, Majlath Z, Fulop F, Toldi J, Vecsei L. Brain aging and disorders of the central nervous system: kynurenines and drug metabolism. Curr Drug Metab (2016) 17:412-29. doi:10.2174/1389200217666151222155043

14. Guillemin GJ, Cullen KM, Lim CK, Smythe GA, Garner B, Kapoor V, et al. Characterization of the kynurenine pathway in human neurons. J Neurosci (2007) 27:12884-92. doi:10.1523/JNEUROSCI.4101-07.2007

15. Platten M, Von Knebel Doeberitz N, Oezen I, Wick W, Ochs K. Cancer immunotherapy by targeting IDO1/TDO and their downstream effectors. Front Immunol (2014) 5:673. doi:10.3389/fimmu.2014.00673 
16. Ball HJ, Sanchez-Perez A, Weiser S, Austin CJ, Astelbauer F, Miu J, et al. Characterization of an indoleamine 2,3-dioxygenase-like protein found in humans and mice. Gene (2007) 396:203-13. doi:10.1016/j.gene.2007.04.010

17. Prendergast GC, Metz R, Muller AJ, Merlo LM, Mandik-Nayak L. IDO2 in immunomodulation and autoimmune disease. Front Immunol (2014) 5:585. doi:10.3389/fimmu.2014.00585

18. Vecsei L, Szalardy L, Fulop F, Toldi J. Kynurenines in the CNS: recent advances and new questions. Nat Rev Drug Discov (2013) 12:64-82. doi:10.1038/ $\operatorname{nrd} 3793$

19. Chen Y, Stankovic R, Cullen KM, Meininger V, Garner B, Coggan S, et al. The kynurenine pathway and inflammation in amyotrophic lateral sclerosis. Neurotox Res (2010) 18:132-42. doi:10.1007/s12640-009-9129-7

20. Sundaram G, Brew BJ, Jones SP, Adams S, Lim CK, Guillemin GJ. Quinolinic acid toxicity on oligodendroglial cells: relevance for multiple sclerosis and therapeutic strategies. J Neuroinflammation (2014) 11:204. doi:10.1186/ s12974-014-0204-5

21. Guillemin GJ, Kerr SJ, Pemberton LA, Smith DG, Smythe GA, Armati PJ, et al. IFN-betalb induces kynurenine pathway metabolism in human macrophages: potential implications for multiple sclerosis treatment. J Interferon Cytokine Res (2001) 21:1097-101. doi:10.1089/107999001317205231

22. Guillemin GJ, Smith DG, Kerr SJ, Smythe GA, Kapoor V, Armati PJ, et al. Characterisation of kynurenine pathway metabolism in human astrocytes and implications in neuropathogenesis. Redox Rep (2000) 5:108-11. doi:10.1179/135100000101535375

23. Guillemin GJ, Smith DG, Smythe GA, Armati PJ, Brew BJ. Expression of the kynurenine pathway enzymes in human microglia and macrophages. Adv Exp Med Biol (2003) 527:105-12. doi:10.1007/978-1-4615-0135-0_12

24. Guillemin GJ, Smythe G, Takikawa O, Brew BJ. Expression of indoleamine 2,3-dioxygenase and production of quinolinic acid by human microglia, astrocytes, and neurons. Glia (2004) 49:15-23. doi:10.1002/glia.20090

25. Guillemin GJ, Kerr SJ, Smythe GA, Smith DG, Kapoor V, Armati PJ, et al. Kynurenine pathway metabolism in human astrocytes: a paradox for neuronal protection. J Neurochem (2001) 78:842-53. doi:10.1046/j.14714159.2001.00498.x

26. Lestage J, Verrier D, Palin K, Dantzer R. The enzyme indoleamine 2,3-dioxygenase is induced in the mouse brain in response to peripheral administration of lipopolysaccharide and superantigen. Brain Behav Immun (2002) 16:596-601. doi:10.1016/S0889-1591(02)00014-4

27. Hassanain HH, Chon SY, Gupta SL. Differential regulation of human indoleamine 2,3-dioxygenase gene expression by interferons-gamma and -alpha. Analysis of the regulatory region of the gene and identification of an interferon-gamma-inducible DNA-binding factor. J Biol Chem (1993) 268:5077-84.

28. Babcock TA, Carlin JM. Transcriptional activation of indoleamine dioxygenase by interleukin 1 and tumor necrosis factor alpha in interferon-treated epithelial cells. Cytokine (2000) 12:588-94. doi:10.1006/cyto.1999.0661

29. Grohmann U, Orabona C, Fallarino F, Vacca C, Calcinaro F, Falorni A, et al. CTLA-4-Ig regulates tryptophan catabolism in vivo. Nat Immunol (2002) 3:1097-101. doi:10.1038/ni846

30. McIlroy D, Tanguy-Royer S, Le Meur N, Guisle I, Royer PJ, Leger J, et al. Profiling dendritic cell maturation with dedicated microarrays. J Leukoc Biol (2005) 78:794-803. doi:10.1189/jlb.0105029

31. Fujigaki S, Saito K, Sekikawa K, Tone S, Takikawa O, Fujii H, et al. Lipopolysaccharide induction of indoleamine 2,3-dioxygenase is mediated dominantly by an IFN-gamma-independent mechanism. Eur J Immunol (2001) 31:2313-8. doi:10.1002/1521-4141(200108)31:8<2313::AID-IMMU2313>3.0.CO;2-S

32. Hayashi T, Beck L, Rossetto C, Gong X, Takikawa O, Takabayashi K, et al. Inhibition of experimental asthma by indoleamine 2,3-dioxygenase. J Clin Invest (2004) 114:270-9. doi:10.1172/JCI21275

33. Hissong BD, Carlin JM. Potentiation of interferon-induced indoleamine 2,3-dioxygenase mRNA in human mononuclear phagocytes by lipopolysaccharide and interleukin-1. J Interferon Cytokine Res (1997) 17:387-93. doi:10.1089/jir.1997.17.387

34. Currier AR, Ziegler MH, Riley MM, Babcock TA, Telbis VP, Carlin JM. Tumor necrosis factor-alpha and lipopolysaccharide enhance interferon-induced antichlamydial indoleamine dioxygenase activity independently. J Interferon Cytokine Res (2000) 20:369-76. doi:10.1089/107999000312306

35. Robinson CM, Shirey KA, Carlin JM. Synergistic transcriptional activation of indoleamine dioxygenase by IFN-gamma and tumor necrosis factor-alpha. J Interferon Cytokine Res (2003) 23:413-21. doi:10.1089/10799 9003322277829

36. Pemberton LA, Kerr SJ, Smythe G, Brew BJ. Quinolinic acid production by macrophages stimulated with IFN-gamma, TNF-alpha, and IFN-alpha. J Interferon Cytokine Res (1997) 17:589-95. doi:10.1089/jir.1997.17.589

37. Alberati-Giani D, Ricciardi-Castagnoli P, Kohler C, Cesura AM. Regulation of the kynurenine metabolic pathway by interferon-gamma in murine cloned macrophages and microglial cells. J Neurochem (1996) 66:996-1004. doi:10.1046/j.1471-4159.1996.66030996.x

38. Heyes MP, Chen CY, Major EO, Saito K. Different kynurenine pathway enzymes limit quinolinic acid formation by various human cell types. Biochem J (1997) 326(Pt 2):351-6. doi:10.1042/bj3260351

39. Heyes MP, Saito K, Lackner A, Wiley CA, Achim CL, Markey SP. Sources of the neurotoxin quinolinic acid in the brain of HIV-1-infected patients and retrovirus-infected macaques. FASEB J (1998) 12:881-96.

40. Zunszain PA, Anacker C, Cattaneo A, Choudhury S, Musaelyan K, Myint AM, et al. Interleukin-1beta: a new regulator of the kynurenine pathway affecting human hippocampal neurogenesis. Neuropsychopharmacology (2012) 37: 939-49. doi:10.1038/npp.2011.277

41. Bosco MC, Rapisarda A, Massazza S, Melillo G, Young H, Varesio L. The tryptophan catabolite picolinic acid selectively induces the chemokines macrophage inflammatory protein-1 alpha and -1 beta in macrophages. J Immunol (2000) 164:3283-91. doi:10.4049/jimmunol.164.6.3283

42. Melillo G, Cox GW, Radzioch D, Varesio L. Picolinic acid, a catabolite of L-tryptophan, is a costimulus for the induction of reactive nitrogen intermediate production in murine macrophages. J Immunol (1993) 150:4031-40.

43. Melillo G, Cox GW, Biragyn A, Sheffler LA, Varesio L. Regulation of nitric-oxide synthase mRNA expression by interferon-gamma and picolinic acid. J Biol Chem (1994) 269:8128-33.

44. Law RO. Regulation of mammalian brain cell volume. J Exp Zool (1994) 268:90-6. doi:10.1002/jez.1402680204

45. Simon MJ, Iliff JJ. Regulation of cerebrospinal fluid (CSF) flow in neurodegenerative, neurovascular and neuroinflammatory disease. Biochim Biophys Acta (2015) 1862(3):442-51. doi:10.1016/j.bbadis.2015.10.014

46. Weller RO. Pathology of cerebrospinal fluid and interstitial fluid of the CNS: significance for Alzheimer disease, prion disorders and multiple sclerosis. J Neuropathol Exp Neurol (1998) 57:885-94. doi:10.1097/00005072199810000-00001

47. Strazielle N, Ghersi-Egea JF. Choroid plexus in the central nervous system: biology and physiopathology. J Neuropathol Exp Neurol (2000) 59:561-74. doi:10.1093/jnen/59.7.561

48. Del Bigio MR. The ependyma: a protective barrier between brain and cerebrospinal fluid. Glia (1995) 14:1-13. doi:10.1002/glia.440140102

49. Vigh-Teichmann I, Vigh B. The cerebrospinal fluid-contacting neuron: a peculiar cell type of the central nervous system. Immunocytochemical aspects. Arch Histol Cytol (1989) 52(Suppl):195-207. doi:10.1679/aohc.52. Suppl_195

50. Brinker T, Stopa E, Morrison J, Klinge P. A new look at cerebrospinal fluid circulation. Fluids Barriers CNS (2014) 11:10. doi:10.1186/2045-8118-11-10

51. Marques F, Sousa JC. The choroid plexus is modulated by various peripheral stimuli: implications to diseases of the central nervous system. Front Cell Neurosci (2015) 9:136. doi:10.3389/fncel.2015.00136

52. Kuenz B, Lutterotti A, Ehling R, Gneiss C, Haemmerle M, Rainer C, et al. Cerebrospinal fluid B cells correlate with early brain inflammation in multiple sclerosis. PLoS One (2008) 3:e2559. doi:10.1371/journal. pone.0002559

53. Fritzsching B, Haas J, Konig F, Kunz P, Fritzsching E, Poschl J, et al. Intracerebral human regulatory T cells: analysis of CD4+ CD25+ FOXP3+ $\mathrm{T}$ cells in brain lesions and cerebrospinal fluid of multiple sclerosis patients. PLoS One (2011) 6:e17988. doi:10.1371/journal.pone.0017988

54. Johanson CE, Duncan JA, Stopa EG, Baird A. Enhanced prospects for drug delivery and brain targeting by the choroid plexus-CSF route. Pharm Res (2005) 22:1011-37. doi:10.1007/s11095-005-6039-0

55. Reiber H. Cerebrospinal fluid - physiology, analysis and interpretation of protein patterns for diagnosis of neurological diseases. Mult Scler (1998) 4:99-107. doi:10.1177/135245859800400302

56. Cepok S, Jacobsen M, Schock S, Omer B, Jaekel S, Boddeker I, et al. Patterns of cerebrospinal fluid pathology correlate with disease progression in multiple sclerosis. Brain (2001) 124:2169-76. doi:10.1093/brain/124.11.2169 
57. Guillemin G, Brew BJ, Noonan CE, Knight TG, Smythe G, Cullen KM. Mass spectrometric detection of quinolinic acid in microdissected Alzheimer's disease plaques. Int Congr Ser (2007) 1304:404-8. doi:10.1016/j.ics.2007.07.012

58. Chen Y, Guillemin GJ. Kynurenine pathway metabolites in humans: disease and healthy states. Int J Tryptophan Res (2009) 2:1-19.

59. Guillemin GJ, Williams KR, Smith DG, Smythe GA, Croitoru-Lamoury J, Brew BJ. Quinolinic acid in the pathogenesis of Alzheimer's disease. Adv Exp Med Biol (2003) 527:167-76. doi:10.1007/978-1-4615-0135-0_19

60. Kerr SJ, Armati PJ, Guillemin GJ, Brew BJ. Chronic exposure of human neurons to quinolinic acid results in neuronal changes consistent with AIDS dementia complex. AIDS (1998) 12:355-63. doi:10.1097/00002030-199804000-00003

61. Kerr SJ, Armati PJ, Pemberton LA, Smythe G, Tattam B, Brew BJ. Kynurenine pathway inhibition reduces neurotoxicity of HIV-1-infected macrophages. Neurology (1997) 49:1671-81. doi:10.1212/WNL.49.6.1671

62. Lim CK, Smythe G, Stocker R, Brew BJ, Guillemin GJ. Characterization of the kynurenine pathway in primary human oligodendrocytes. Int Congr Ser (2007) 1304:213-7. doi:10.1016/j.ics.2007.07.011

63. Owe-Young R, Webster NL, Mukhtar M, Pomerantz RJ, Smythe G, Walker D, et al. Kynurenine pathway metabolism in human blood-brain-barrier cells: implications for immune tolerance and neurotoxicity. J Neurochem (2008) 105:1346-57. doi:10.1111/j.1471-4159.2008.05241.x

64. Komori M, Blake A, Greenwood M, Lin YC, Kosa P, Ghazali D, et al. Cerebrospinal fluid markers reveal intrathecal inflammation in progressive multiple sclerosis. Ann Neurol (2015) 78:3-20. doi:10.1002/ana.24408

65. Villar LM, Picon C, Costa-Frossard L, Alenda R, Garcia-Caldentey J, Espino M, et al. Cerebrospinal fluid immunological biomarkers associated with axonal damage in multiple sclerosis. Eur J Neurol (2015) 22:1169-75. doi:10.1111/ene.12579

66. Modvig S, Degn M, Roed H, Sorensen TL, Larsson H, Langkilde AR, et al. Cerebrospinal fluid levels of chitinase 3-like 1 and neurofilament light chain predict multiple sclerosis development and disability after optic neuritis. Mult Scler (2015) 21:1761-70. doi:10.1177/1352458515574148

67. Flanagan EM, Erickson JB, Viveros OH, Chang SY, Reinhard JF Jr. Neurotoxin quinolinic acid is selectively elevated in spinal cords of rats with experimental allergic encephalomyelitis. J Neurochem (1995) 64:1192-6. doi:10.1046/j.1471-4159.1995.64031192.x

68. Sakurai K, Zou JP, Tschetter JR, Ward JM, Shearer GM. Effect of indoleamine 2,3-dioxygenase on induction of experimental autoimmune encephalomyelitis. J Neuroimmunol (2002) 129:186-96. doi:10.1016/S0165-5728(02)00176-5

69. Monaco F, Fumero S, Mondino A, Mutani R. Plasma and cerebrospinal fluid tryptophan in multiple sclerosis and degenerative diseases. J Neurol Neurosurg Psychiatry (1979) 42:640-1. doi:10.1136/jnnp.42.7.640

70. Rejdak K, Bartosik-Psujek H, Dobosz B, Kocki T, Grieb P, Giovannoni G, et al. Decreased level of kynurenic acid in cerebrospinal fluid of relapsing-onset multiple sclerosis patients. Neurosci Lett (2002) 331:63-5. doi:10.1016/ S0304-3940(02)00710-3

71. Rejdak K, Petzold A, Kocki T, Kurzepa J, Grieb P, Turski WA, et al. Astrocytic activation in relation to inflammatory markers during clinical exacerbation of relapsing-remitting multiple sclerosis. J Neural Transm (Vienna) (2007) 114:1011-5. doi:10.1007/s00702-007-0667-y

72. Hartai Z, Klivenyi P, Janaky T, Penke B, Dux L, Vecsei L. Kynurenine metabolism in multiple sclerosis. Acta Neurol Scand (2005) 112:93-6. doi:10.1111/j.1600-0404.2005.00442.x

73. Turski MP, Turska M, Paluszkiewicz P, Parada-Turska J, Oxenkrug GF. Kynurenic acid in the digestive system-new facts, new challenges. Int J Tryptophan Res (2013) 6:47-55. doi:10.4137/IJTR.S12536

74. Aeinehband S, Brenner P, Stahl S, Bhat M, Fidock MD, Khademi M, et al. Cerebrospinal fluid kynurenines in multiple sclerosis; relation to disease course and neurocognitive symptoms. Brain Behav Immun (2016) 51:47-55. doi:10.1016/j.bbi.2015.07.016

75. Hedegaard CJ, Chen N, Sellebjerg F, Sorensen PS, Leslie RG, Bendtzen K, et al. Autoantibodies to myelin basic protein (MBP) in healthy individuals and in patients with multiple sclerosis: a role in regulating cytokine responses to MBP. Immunology (2009) 128:e451-61. doi:10.1111/j.1365-2567.2008.02999.x

76. Mancuso R, Hernis A, Agostini S, Rovaris M, Caputo D, Fuchs D, et al. Indoleamine 2,3 dioxygenase (IDO) expression and activity in relapsingremitting multiple sclerosis. PLoS One (2015) 10:e0130715. doi:10.1371/ journal.pone. 0130715
77. Sadowska-Bartosz I, Adamczyk-Sowa M, Gajewska A, Bartosz G. Oxidative modification of blood serum proteins in multiple sclerosis after interferon or mitoxantrone treatment. J Neuroimmunol (2014) 266:67-74. doi:10.1016/j. jneuroim.2013.11.005

78. Ransohoff RM, Kivisakk P, Kidd G. Three or more routes for leukocyte migration into the central nervous system. Nat Rev Immunol (2003) 3:569-81. doi:10.1038/nri1130

79. Sedgwick JD, Hughes CC, Male DK, Macphee IA, Ter Meulen V. Antigenspecific damage to brain vascular endothelial cells mediated by encephalitogenic and nonencephalitogenic CD4+ T cell lines in vitro. J Immunol (1990) 145:2474-81.

80. Hu P, Pollard JD, Chan-Ling T. Breakdown of the blood-retinal barrier induced by activated T cells of nonneural specificity. Am J Pathol (2000) 156:1139-49. doi:10.1016/S0002-9440(10)64982-6

81. Smorodchenko A, Wuerfel J, Pohl EE, Vogt J, Tysiak E, Glumm R, et al. CNS-irrelevant T-cells enter the brain, cause blood-brain barrier disruption but no glial pathology. Eur JNeurosci (2007) 26:1387-98. doi:10.1111/j.1460-9568.2007.05792.x

82. Larochelle C, Alvarez JI, Prat A. How do immune cells overcome the blood-brain barrier in multiple sclerosis? FEBS Lett (2011) 585:3770-80. doi:10.1016/j.febslet.2011.04.066

83. Lopes Pinheiro MA, Kooij G, Mizee MR, Kamermans A, Enzmann G, Lyck R, et al. Immune cell trafficking across the barriers of the central nervous system in multiple sclerosis and stroke. Biochim Biophys Acta (2015) 1862(3):461-71. doi:10.1016/j.bbadis.2015.10.018

84. Hu P, Pollard J, Hunt N, Chan-Ling T. Microvascular and cellular responses in the retina of rats with acute experimental allergic encephalomyelitis (EAE). Brain Pathol (1998) 8:487-98. doi:10.1111/j.1750-3639.1998.tb00170.x

85. Runge VM, Schoerner W, Niendorf HP, Laniado M, Koehler D, Claussen C, et al. Initial clinical evaluation of gadolinium DTPA for contrast-enhanced magnetic resonance imaging. Magn Reson Imaging (1985) 3:27-35. doi:10.1016/0730-725X(85)90006-2

86. Miller DH, Rudge P, Johnson G, Kendall BE, Macmanus DG, Moseley IF, et al. Serial gadolinium enhanced magnetic resonance imaging in multiple sclerosis. Brain (1988) 111(Pt 4):927-39. doi:10.1093/brain/111.4.927

87. Kermode AG, Thompson AJ, Tofts P, Macmanus DG, Kendall BE, Kingsley DP, et al. Breakdown of the blood-brain barrier precedes symptoms and other MRI signs of new lesions in multiple sclerosis. Pathogenetic and clinical implications. Brain (1990) 113(Pt 5):1477-89. doi:10.1093/brain/113.5.1477

88. Claudio L, Kress Y, Factor J, Brosnan CF. Mechanisms of edema formation in experimental autoimmune encephalomyelitis. The contribution of inflammatory cells. Am J Pathol (1990) 137:1033-45.

89. Lossinsky AS, Badmajew V, Robson JA, Moretz RC, Wisniewski HM. Sites of egress of inflammatory cells and horseradish peroxidase transport across the blood-brain barrier in a murine model of chronic relapsing experimental allergic encephalomyelitis. Acta Neuropathol (1989) 78:359-71. doi:10.1007/ BF00688172

90. Goodkin DE, Rooney WD, Sloan R, Bacchetti P, Gee L, Vermathen M, et al. A serial study of new MS lesions and the white matter from which they arise. Neurology (1998) 51:1689-97. doi:10.1212/WNL.51.6.1689

91. Kirk J, Plumb J, Mirakhur M, McQuaid S. Tight junctional abnormality in multiple sclerosis white matter affects all calibres of vessel and is associated with blood-brain barrier leakage and active demyelination. J Pathol (2003) 201:319-27. doi:10.1002/path.1434

92. McQuaid S, Cunnea P, McMahon J, Fitzgerald U. The effects of blood-brain barrier disruption on glial cell function in multiple sclerosis. Biochem Soc Trans (2009) 37:329-31. doi:10.1042/BST0370329

93. Plumb J, McQuaid S, Mirakhur M, Kirk J. Abnormal endothelial tight junctions in active lesions and normal-appearing white matter in multiple sclerosis. Brain Pathol (2002) 12:154-69. doi:10.1111/j.1750-3639.2002.tb00430.x

94. Vos CM, Geurts JJ, Montagne L, Van Haastert ES, Bo L, Van Der Valk P, et al. Blood-brain barrier alterations in both focal and diffuse abnormalities on postmortem MRI in multiple sclerosis. Neurobiol Dis (2005) 20:953-60. doi:10.1016/j.nbd.2005.06.012

95. Filippi M, Rocca MA, Martino G, Horsfield MA, Comi G. Magnetization transfer changes in the normal appearing white matter precede the appearance of enhancing lesions in patients with multiple sclerosis. Ann Neurol (1998) 43:809-14. doi:10.1002/ana.410430616 
96. Cramer SP, Simonsen H, Frederiksen JL, Rostrup E, Larsson HB. Abnormal blood-brain barrier permeability in normal appearing white matter in multiple sclerosis investigated by MRI. Neuroimage Clin (2014) 4:182-9. doi:10.1016/j.nicl.2013.12.001

97. Ingrisch M, Sourbron S, Morhard D, Ertl-Wagner B, Kumpfel T, Hohlfeld R, et al. Quantification of perfusion and permeability in multiple sclerosis: dynamic contrast-enhanced MRI in 3D at 3T. Invest Radiol (2012) 47:252-8. doi:10.1097/RLI.0b013e31823bfc97

98. Atkins EJ, Biousse V, Newman NJ. The natural history of optic neuritis. Rev Neurol Dis (2006) 3:45-56.

99. Cramer SP, Modvig S, Simonsen HJ, Frederiksen JL, Larsson HB. Permeability of the blood-brain barrier predicts conversion from optic neuritis to multiple sclerosis. Brain (2015) 138:2571-83. doi:10.1093/brain/awv203

100. Nathoo N, Jalal H, Natah S, Zhang Q, Wu Y, Dunn J. Hypoxia and inflammation-induced disruptions of the blood-brain and bloodcerebrospinal fluid barriers assessed using a novel T1-based MRI method. Acta Neurochir Suppl (2016) 121:23-8. doi:10.1007/978-3-319-18497-5_5

101. Kawakami N, Flugel A. Knocking at the brain's door: intravital two-photon imaging of autoreactive $\mathrm{T}$ cell interactions with CNS structures. Semin Immunopathol (2010) 32:275-87. doi:10.1007/s00281-010-0216-X

102. Mandi Y, Vecsei L. The kynurenine system and immunoregulation. J Neural Transm (Vienna) (2012) 119:197-209. doi:10.1007/s00702-011-0681-y

103. Bozza S, Fallarino F, Pitzurra L, Zelante T, Montagnoli C, Bellocchio S, et al. A crucial role for tryptophan catabolism at the host/Candida albicans interface. J Immunol (2005) 174:2910-8. doi:10.4049/jimmunol.174.5.2910

104. Munn DH, Shafizadeh E, Attwood JT, Bondarev I, Pashine A, Mellor AL. Inhibition of $\mathrm{T}$ cell proliferation by macrophage tryptophan catabolism. J Exp Med (1999) 189:1363-72. doi:10.1084/jem.189.9.1363

105. Frumento G, Rotondo R, Tonetti M, Damonte G, Benatti U, Ferrara GB. Tryptophan-derived catabolites are responsible for inhibition of $\mathrm{T}$ and natural killer cell proliferation induced by indoleamine 2,3-dioxygenase. J Exp Med (2002) 196:459-68. doi:10.1084/jem.20020121

106. Terness P, Bauer TM, Rose L, Dufter C, Watzlik A, Simon H, et al. Inhibition of allogeneic $\mathrm{T}$ cell proliferation by indoleamine 2,3-dioxygenase-expressing dendritic cells: mediation of suppression by tryptophan metabolites. J Exp Med (2002) 196:447-57. doi:10.1084/jem.20020052

107. Fallarino F, Grohmann U, Vacca C, Bianchi R, Orabona C, Spreca A, et al. T cell apoptosis by tryptophan catabolism. Cell Death Differ (2002) 9:1069-77. doi:10.1038/sj.cdd.4401073

108. Bauer TM, Jiga LP, Chuang JJ, Randazzo M, Opelz G, Terness P. Studying the immunosuppressive role of indoleamine 2,3-dioxygenase: tryptophan metabolites suppress rat allogeneic T-cell responses in vitro and in vivo. Transpl Int (2005) 18:95-100. doi:10.1111/j.1432-2277.2004.00031.x

109. Della Chiesa M, Carlomagno S, Frumento G, Balsamo M, Cantoni C, Conte R, et al. The tryptophan catabolite L-kynurenine inhibits the surface expression of NKp46- and NKG2D-activating receptors and regulates NK-cell function. Blood (2006) 108:4118-25. doi:10.1182/blood-200603-006700

110. Fallarino F, Grohmann U, You S, McGrath BC, Cavener DR, Vacca C, et al. The combined effects of tryptophan starvation and tryptophan catabolites down-regulate $\mathrm{T}$ cell receptor zeta-chain and induce a regulatory phenotype in naive T cells. J Immunol (2006) 176:6752-61. doi:10.4049/ jimmunol.176.11.6752

111. Pallotta MT, Orabona C, Volpi C, Vacca C, Belladonna ML, Bianchi R, et al. Indoleamine 2,3-dioxygenase is a signaling protein in long-term tolerance by dendritic cells. Nat Immunol (2011) 12:870-8. doi:10.1038/ni.2077

112. Belladonna ML, Grohmann U, Guidetti P, Volpi C, Bianchi R, Fioretti MC, et al. Kynurenine pathway enzymes in dendritic cells initiate tolerogenesis in the absence of functional IDO. J Immunol (2006) 177:130-7. doi:10.4049/ jimmunol.177.1.130

113. Colin-Gonzalez AL, Maldonado PD, Santamaria A. 3-Hydroxykynurenine: an intriguing molecule exerting dual actions in the central nervous system. Neurotoxicology (2013) 34:189-204. doi:10.1016/j.neuro.2012.11.007

114. Aranami T, Yamamura T. Th17 cells and autoimmune encephalomyelitis (EAE/MS). Allergol Int (2008) 57:115-20. doi:10.2332/allergolint.R-07-159

115. Yan Y, Zhang GX, Gran B, Fallarino F, Yu S, Li H, et al. IDO upregulates regulatory $\mathrm{T}$ cells via tryptophan catabolite and suppresses encephalitogenic $\mathrm{T}$ cell responses in experimental autoimmune encephalomyelitis. J Immunol (2010) 185:5953-61. doi:10.4049/jimmunol.1001628
116. Kwidzinski E, Bunse J, Aktas O, Richter D, Mutlu L, Zipp F, et al. Indolamine 2,3-dioxygenase is expressed in the CNS and down-regulates autoimmune inflammation. FASEB J (2005) 19:1347-9. doi:10.1096/fj.04-3228fje

117. Xiao BG, Wu XC, Yang JS, Xu LY, Liu X, Huang YM, et al. Therapeutic potential of IFN-gamma-modified dendritic cells in acute and chronic experimental allergic encephalomyelitis. Int Immunol (2004) 16:13-22. doi:10.1093/intimm/dxh003

118. Chiarugi A, Cozzi A, Ballerini C, Massacesi L, Moroni F. Kynurenine 3-mono-oxygenase activity and neurotoxic kynurenine metabolites increase in the spinal cord of rats with experimental allergic encephalomyelitis. Neuroscience (2001) 102:687-95. doi:10.1016/S0306-4522(00)00504-2

119. Rajda C, Majlath Z, Pukoli D, Vecsei L. Kynurenines and multiple sclerosis: the dialogue between the immune system and the central nervous system. Int J Mol Sci (2015) 16:18270-82. doi:10.3390/ijms160818270

120. Ott M, Demisch L, Engelhardt W, Fischer PA. Interleukin-2, soluble interleukin-2-receptor, neopterin, L-tryptophan and beta 2-microglobulin levels in CSF and serum of patients with relapsing-remitting or chronic-progressive multiple sclerosis. J Neurol (1993) 241:108-14. doi:10.1007/BF00869773

121. Kwidzinski E, Bechmann I. IDO expression in the brain: a double-edged sword. J Mol Med (Berl) (2007) 85:1351-9. doi:10.1007/s00109-007-0229-7

122. Auffray C, Fogg D, Garfa M, Elain G, Join-Lambert O, Kayal S, et al. Monitoring of blood vessels and tissues by a population of monocytes with patrolling behavior. Science (2007) 317:666-70. doi:10.1126/science.1142883

123. Mitchell AJ, Roediger B, Weninger W. Monocyte homeostasis and the plasticity of inflammatory monocytes. Cell Immunol (2014) 291:22-31. doi:10.1016/j.cellimm.2014.05.010

124. King IL, Dickendesher TL, Segal BM. Circulating Ly-6C+ myeloid precursors migrate to the CNS and play a pathogenic role during autoimmune demyelinating disease. Blood (2009) 113:3190-7. doi:10.1182/blood-2008-07-168575

125. Ajami B, Bennett JL, Krieger C, McNagny KM, Rossi FM. Infiltrating monocytes trigger EAE progression, but do not contribute to the resident microglia pool. Nat Neurosci (2011) 14:1142-9. doi:10.1038/nn.2887

126. Mishra MK, Wang J, Silva C, Mack M, Yong VW. Kinetics of proinflammatory monocytes in a model of multiple sclerosis and its perturbation by laquinimod. Am J Pathol (2012) 181:642-51. doi:10.1016/j.ajpath.2012.05.011

127. Moreno MA, Burns T, Yao P, Miers L, Pleasure D, Soulika AM. Therapeutic depletion of monocyte-derived cells protects from long-term axonal loss in experimental autoimmune encephalomyelitis. J Neuroimmunol (2016) 290:36-46. doi:10.1016/j.jneuroim.2015.11.004

128. Ferguson B, Matyszak MK, Esiri MM, Perry VH. Axonal damage in acute multiple sclerosis lesions. Brain (1997) 120(Pt 3):393-9. doi:10.1093/ brain/120.3.393

129. Trapp BD, Peterson J, Ransohoff RM, Rudick R, Mork S, Bo L. Axonal transection in the lesions of multiple sclerosis. N Engl J Med (1998) 338:278-85. doi:10.1056/NEJM199801293380502

130. Trebst C, Sorensen TL, Kivisakk P, Cathcart MK, Hesselgesser J, Horuk R, et al. CCR1+/CCR5+ mononuclear phagocytes accumulate in the central nervous system of patients with multiple sclerosis. Am JPathol (2001) 159:1701-10. doi:10.1016/S0002-9440(10)63017-9

131. Yamasaki R, Lu H, Butovsky O, Ohno N, Rietsch AM, Cialic R, et al. Differential roles of microglia and monocytes in the inflamed central nervous system. J Exp Med (2014) 211:1533-49. doi:10.1084/jem.20132477

132. Braidy N, Grant R, Adams S, Brew BJ, Guillemin GJ. Mechanism for quinolinic acid cytotoxicity in human astrocytes and neurons. Neurotox Res (2009) 16:77-86. doi:10.1007/s12640-009-9051-z

133. Arellano G, Ottum PA, Reyes LI, Burgos PI, Naves R. Stage-specific role of interferon-gamma in experimental autoimmune encephalomyelitis and multiple sclerosis. Front Immunol (2015) 6:492. doi:10.3389/fimmu.2015.00492

134. Guillemin GJ, Brew BJ, Noonan CE, Takikawa O, Cullen KM. Indoleamine 2,3 dioxygenase and quinolinic acid immunoreactivity in Alzheimer's disease hippocampus. Neuropathol Appl Neurobiol (2005) 31:395-404. doi:10.1111/j.1365-2990.2005.00655.x

135. Wu W, Nicolazzo JA, Wen L, Chung R, Stankovic R, Bao SS, et al. Expression of tryptophan 2,3-dioxygenase and production of kynurenine pathway metabolites in triple transgenic mice and human Alzheimer's disease brain. PLoS One (2013) 8:e59749. doi:10.1371/journal.pone.0059749

136. Chen Y, Brew BJ, Guillemin GJ. Characterization of the kynurenine pathway in NSC-34 cell line: implications for amyotrophic lateral sclerosis. J Neurochem (2011) 118:816-25. doi:10.1111/j.1471-4159.2010.07159.x 
137. Schwarcz R, Bruno JP, Muchowski PJ, Wu HQ. Kynurenines in the mammalian brain: when physiology meets pathology. Nat Rev Neurosci (2012) 13:465-77. doi:10.1038/nrn3257

138. Chiarugi A, Meli E, Moroni F. Similarities and differences in the neuronal death processes activated by $3 \mathrm{OH}-$ kynurenine and quinolinic acid. J Neurochem (2001) 77:1310-8. doi:10.1046/j.1471-4159.2001.00335.x

139. Guillemin GJ. Quinolinic acid, the inescapable neurotoxin. FEBS J (2012) 279:1356-65. doi:10.1111/j.1742-4658.2012.08485.x

140. Foster AC, Okuno E, Brougher DS, Schwarcz R. A radioenzymatic assay for quinolinic acid. Anal Biochem (1986) 158:98-103. doi:10.1016/00032697(86)90595-6

141. Guillemin GJ. Quinolinic acid: neurotoxicity. FEBS J (2012) 279:1355. doi:10.1111/j.1742-4658.2012.08493.x

142. Braidy N, Guillemin GJ, Grant R. Effects of kynurenine pathway inhibition on NAD metabolism and cell viability in human primary astrocytes and neurons. Int J Tryptophan Res (2011) 4:29-37. doi:10.4137/IJTR.S7052

143. Lekieffre D, Plotkine M, Allix M, Boulu RG. Kynurenic acid antagonizes hippocampal quinolinic acid neurotoxicity: behavioral and histological evaluation. Neurosci Lett (1990) 120:31-3. doi:10.1016/0304-3940(90)90160-B

144. Sas K, Robotka H, Toldi J, Vecsei L. Mitochondria, metabolic disturbances, oxidative stress and the kynurenine system, with focus on neurodegenerative disorders. J Neurol Sci (2007) 257:221-39. doi:10.1016/j.jns.2007.01.033

145. Mehta A, Prabhakar M, Kumar P, Deshmukh R, Sharma PL. Excitotoxicity: bridge to various triggers in neurodegenerative disorders. Eur J Pharmacol (2013) 698:6-18. doi:10.1016/j.ejphar.2012.10.032

146. Zhao C, Deng W, Gage FH. Mechanisms and functional implications of adult neurogenesis. Cell (2008) 132:645-60. doi:10.1016/j.cell.2008.01.033

147. Croitoru-Lamoury J, Lamoury FM, Caristo M, Suzuki K, Walker D, Takikawa $\mathrm{O}$, et al. Interferon-gamma regulates the proliferation and differentiation of mesenchymal stem cells via activation of indoleamine 2,3 dioxygenase (IDO). PLoS One (2011) 6:e14698. doi:10.1371/journal.pone.0014698

148. Grohmann U, Bronte V. Control of immune response by amino acid metabolism. Immunol Rev (2010) 236:243-64. doi:10.1111/j.1600-065X.2010.00915.x

149. Lugo-Huitron R, Ugalde Muniz P, Pineda B, Pedraza-Chaverri J, Rios C, Perez-De La Cruz V. Quinolinic acid: an endogenous neurotoxin with multiple targets. Oxid Med Cell Longev (2013) 2013:104024. doi:10.1155/2013/104024

150. Sekine A, Okamoto M, Kanatani Y, Sano M, Shibata K, Fukuwatari T. Amino acids inhibit kynurenic acid formation via suppression of kynurenine uptake or kynurenic acid synthesis in rat brain in vitro. Springerplus (2015) 4:48. doi:10.1186/s40064-015-0826-9

151. Cammer W. Oligodendrocyte killing by quinolinic acid in vitro. Brain Res (2001) 896:157-60. doi:10.1016/S0006-8993(01)02017-0

152. Cammer W. Protection of cultured oligodendrocytes against tumor necrosis factor-alpha by the antioxidants coenzyme $\mathrm{Q}(10)$ and $\mathrm{N}$-acetyl cysteine. Brain Res Bull (2002) 58:587-92. doi:10.1016/S0361-9230(02)00830-4

153. Tavares RG, Tasca CI, Santos CE, Wajner M, Souza DO, Dutra-Filho CS. Quinolinic acid inhibits glutamate uptake into synaptic vesicles from rat brain. Neuroreport (2000) 11:249-53. doi:10.1097/00001756-200002070-00005

154. Tavares RG, Tasca CI, Santos CE, Alves LB, Porciuncula LO, Emanuelli T, et al. Quinolinic acid stimulates synaptosomal glutamate release and inhibits glutamate uptake into astrocytes. Neurochem Int (2002) 40:621-7. doi:10.1016/S0197-0186(01)00133-4

155. Piermartiri TC, Vandresen-Filho S, De Araujo Herculano B, Martins WC, Dal'Agnolo D, Stroeh E, et al. Atorvastatin prevents hippocampal cell death due to quinolinic acid-induced seizures in mice by increasing AKT phosphorylation and glutamate uptake. Neurotox Res (2009) 16:106-15. doi:10.1007/s12640-009-9057-6

156. Baverel G, Martin G, Michoudet C. Glutamine synthesis from aspartate in guinea-pig renal cortex. Biochem J(1990) 268:437-42. doi:10.1042/bj2680437

157. Ting KK, Brew BJ, Guillemin GJ. Effect of quinolinic acid on human astrocytes morphology and functions: implications in Alzheimer's disease. J Neuroinflammation (2009) 6:36. doi:10.1186/1742-2094-6-36

158. Goda K, Kishimoto R, Shimizu S, Hamane Y, Ueda M. Quinolinic acid and active oxygens. Possible contribution of active oxygens during cell death in the brain. Adv Exp Med Biol (1996) 398:247-54. doi:10.1007/978-1-46130381-7_38

159. Muller AC, Dairam A, Limson JL, Daya S. Mechanisms by which acyclovir reduces the oxidative neurotoxicity and biosynthesis of quinolinic acid. Life Sci (2007) 80:918-25. doi:10.1016/j.lfs.2006.11.031
160. Rodriguez-Martinez E, Camacho A, Maldonado PD, Pedraza-Chaverri J, Santamaria D, Galvan-Arzate S, et al. Effect of quinolinic acid on endogenous antioxidants in rat corpus striatum. Brain Res (2000) 858:436-9. doi:10.1016/ S0006-8993(99)02474-9

161. Tasset I, Perez-De La Cruz V, Elinos-Calderon D, Carrillo-Mora P, GonzalezHerrera IG, Luna-Lopez A, et al. Protective effect of tert-butylhydroquinone on the quinolinic-acid-induced toxicity in rat striatal slices: role of the NRF2-antioxidant response element pathway. Neurosignals (2010) 18:24-31. doi: $10.1159 / 000243650$

162. Franklin RJ, Ffrench-Constant C, Edgar JM, Smith KJ. Neuroprotection and repair in multiple sclerosis. Nat Rev Neurol (2012) 8:624-34. doi:10.1038/ nrneurol.2012.200

163. Nikic I, Merkler D, Sorbara C, Brinkoetter M, Kreutzfeldt M, Bareyre FM, et al. A reversible form of axon damage in experimental autoimmune encephalomyelitis and multiple sclerosis. Nat Med (2011) 17:495-9. doi:10.1038/ nm.2324

164. Haider L, Fischer MT, Frischer JM, Bauer J, Hoftberger R, Botond G, et al. Oxidative damage in multiple sclerosis lesions. Brain (2011) 134:1914-24. doi:10.1093/brain/awr128

165. Kubicova L, Hadacek F, Chobot V. Quinolinic acid: neurotoxin or oxidative stress modulator? Int J Mol Sci (2013) 14:21328-38. doi:10.3390/ ijms141121328

166. Kubicova L, Hadacek F, Weckwerth W, Chobot V. Effects of endogenous neurotoxin quinolinic acid on reactive oxygen species production by Fenton reaction catalyzed by iron or copper. J Organomet Chem (2015) 782:111-5. doi:10.1016/j.jorganchem.2015.01.030

167. Pierozan P, Zamoner A, Soska AK, Silvestrin RB, Loureiro SO, Heimfarth L, et al. Acute intrastriatal administration of quinolinic acid provokes hyperphosphorylation of cytoskeletal intermediate filament proteins in astrocytes and neurons of rats. Exp Neurol (2010) 224:188-96. doi:10.1016/j. expneurol.2010.03.009

168. Rahman A, Ting K, Cullen KM, Braidy N, Brew BJ, Guillemin GJ. The excitotoxin quinolinic acid induces tau phosphorylation in human neurons. PLoS One (2009) 4:e6344. doi:10.1371/journal.pone.0006344

169. Anderson JM, Hampton DW, Patani R, Pryce G, Crowther RA, Reynolds R, et al. Abnormally phosphorylated tau is associated with neuronal and axonal loss in experimental autoimmune encephalomyelitis and multiple sclerosis. Brain (2008) 131:1736-48. doi:10.1093/brain/awn119

170. Pierozan P, Ferreira F, Ortiz De Lima B, Goncalves Fernandes C, Totarelli Monteforte P, De Castro Medaglia N, et al. The phosphorylation status and cytoskeletal remodeling of striatal astrocytes treated with quinolinic acid. Exp Cell Res (2014) 322:313-23. doi:10.1016/j.yexcr.2014.02.024

171. Pierozan P, Ferreira F, De Lima BO, Pessoa-Pureur R. Quinolinic acid induces disrupts cytoskeletal homeostasis in striatal neurons. Protective role of astrocyte-neuron interaction. J Neurosci Res (2015) 93:268-84. doi:10.1002/ jnr.23494

172. Fernandes AM, Landeira-Fernandez AM, Souza-Santos P, CarvalhoAlves PC, Castilho RF. Quinolinate-induced rat striatal excitotoxicity impairs endoplasmic reticulum Ca2+-ATPase function. Neurochem Res (2008) 33:1749-58. doi:10.1007/s11064-008-9619-7

173. Vamos E, Pardutz A, Klivenyi P, Toldi J, Vecsei L. The role of kynurenines in disorders of the central nervous system: possibilities for neuroprotection. J Neurol Sci (2009) 283:21-7. doi:10.1016/j.jns.2009.02.326

174. Okuda S, Nishiyama N, Saito H, Katsuki H. Hydrogen peroxide-mediated neuronal cell death induced by an endogenous neurotoxin, 3-hydroxykynurenine. Proc Natl Acad Sci U S A (1996) 93:12553-8. doi:10.1073/pnas.93.22.12553

175. Okuda S, Nishiyama N, Saito H, Katsuki H. 3-Hydroxykynurenine, an endogenous oxidative stress generator, causes neuronal cell death with apoptotic features and region selectivity. J Neurochem (1998) 70:299-307. doi:10.1046/j.1471-4159.1998.70010299.x

176. Smith AJ, Smith RA, Stone TW. 5-Hydroxyanthranilic acid, a tryptophan metabolite, generates oxidative stress and neuronal death via $\mathrm{p} 38$ activation in cultured cerebellar granule neurones. Neurotox Res (2009) 15:303-10. doi:10.1007/s12640-009-9034-0

177. Jeong JH, Kim HJ, Lee TJ, Kim MK, Park ES, Choi BS. Epigallocatechin 3 -gallate attenuates neuronal damage induced by 3 -hydroxykynurenine. Toxicology (2004) 195:53-60. doi:10.1016/j.tox.2003.08.007

178. Braidy N, Grant R, Brew BJ, Adams S, Jayasena T, Guillemin GJ. Effects of kynurenine pathway metabolites on intracellular NAD synthesis and cell 
death in human primary astrocytes and neurons. Int J Tryptophan Res (2009) 2:61-9.

179. Berger F, Ramirez-Hernandez MH, Ziegler M. The new life of a centenarian: signalling functions of NAD(P). Trends Biochem Sci (2004) 29:111-8. doi:10.1016/j.tibs.2004.01.007

180. Zhang T, Berrocal JG, Frizzell KM, Gamble MJ, Dumond ME, Krishnakumar R, et al. Enzymes in the NAD+ salvage pathway regulate SIRT1 activity at target gene promoters. J Biol Chem (2009) 284:20408-17. doi:10.1074/jbc.M109.016469

181. Guidetti P, Schwarcz R. 3-Hydroxykynurenine potentiates quinolinate but not NMDA toxicity in the rat striatum. Eur J Neurosci (1999) 11:3857-63. doi:10.1046/j.1460-9568.1999.00806.x

182. Schwarcz R, Guidetti P, Sathyasaikumar KV, Muchowski PJ. Of mice, rats and men: revisiting the quinolinic acid hypothesis of Huntington's disease. Prog Neurobiol (2010) 90:230-45. doi:10.1016/j.pneurobio.2009.04.005

183. Stone TW, Darlington LG. Endogenous kynurenines as targets for drug discovery and development. Nat Rev Drug Discov (2002) 1:609-20. doi:10.1038/ nrd870

184. Darlington LG, Forrest CM, Mackay GM, Smith RA, Smith AJ, Stoy N, et al. On the biological importance of the 3-hydroxyanthranilic acid: anthranilic acid ratio. Int J Tryptophan Res (2010) 3:51-9. doi:10.4137/IJTR.S4282

185. Saito K, Chen CY, Masana M, Crowley JS, Markey SP, Heyes MP. 4-Chloro3-hydroxyanthranilate, 6-chlorotryptophan and norharmane attenuate quinolinic acid formation by interferon-gamma-stimulated monocytes (THP-1 cells). Biochem J (1993) 291(Pt 1):11-4. doi:10.1042/bj2910011

186. Pucci L, Perozzi S, Cimadamore F, Orsomando G, Raffaelli N. Tissue expression and biochemical characterization of human 2-amino 3-carboxymuconate 6-semialdehyde decarboxylase, a key enzyme in tryptophan catabolism. FEBS J (2007) 274:827-40. doi:10.1111/j.1742-4658.2007.05635.x

187. Stone TW, Perkins MN. Quinolinic acid: a potent endogenous excitant at amino acid receptors in CNS. Eur J Pharmacol (1981) 72:411-2. doi:10.1016/0014-2999(81)90587-2

188. Salter MG, Fern R. NMDA receptors are expressed in developing oligodendrocyte processes and mediate injury. Nature (2005) 438:1167-71. doi:10.1038/nature04301

189. Micu I, Jiang Q, Coderre E, Ridsdale A, Zhang L, Woulfe J, et al. NMDA receptors mediate calcium accumulation in myelin during chemical ischaemia. Nature (2006) 439:988-92. doi:10.1038/nature04474

190. Matute C, Alberdi E, Domercq M, Perez-Cerda F, Perez-Samartin A, Sanchez-Gomez MV. The link between excitotoxic oligodendroglial death and demyelinating diseases. Trends Neurosci (2001) 24:224-30. doi:10.1016/ S0166-2236(00)01746-X

191. Williams A. Remyelination in multiple sclerosis: what do we know and where are we going? Neurodegener Dis Manag (2015) 5:49-59. doi:10.2217/ nmt. 14.40

192. Matute C, Alberdi E, Domercq M, Sanchez-Gomez MV, Perez-Samartin A, Rodriguez-Antiguedad A, et al. Excitotoxic damage to white matter. J Anat (2007) 210:693-702. doi:10.1111/j.1469-7580.2007.00733.x

193. Sanchez-Gomez MV, Alberdi E, Ibarretxe G, Torre I, Matute C. Caspasedependent and caspase-independent oligodendrocyte death mediated by AMPA and kainate receptors. J Neurosci (2003) 23:9519-28.

194. McDonald JW, Levine JM, Qu Y. Multiple classes of the oligodendrocyte lineage are highly vulnerable to excitotoxicity. Neuroreport (1998) 9:2757-62. doi:10.1097/00001756-199808240-00014

195. Ziak D, Chvatal A, Sykova E. Glutamate-, kainate- and NMDA-evoked membrane currents in identified glial cells in rat spinal cord slice. Physiol Res (1998) 47:365-75.

196. Karadottir R, Cavelier P, Bergersen LH, Attwell D. NMDA receptors are expressed in oligodendrocytes and activated in ischaemia. Nature (2005) 438:1162-6. doi:10.1038/nature04302

197. Bakiri Y, Hamilton NB, Karadottir R, Attwell D. Testing NMDA receptor block as a therapeutic strategy for reducing ischaemic damage to CNS white matter. Glia (2008) 56:233-40. doi:10.1002/glia.20608

198. de Carvalho LP, Bochet P, Rossier J. The endogenous agonist quinolinic acid and the non endogenous homoquinolinic acid discriminate between NMDAR2 receptor subunits. Neurochem Int (1996) 28:445-52. doi:10.1016/0197-0186(95)00091-7

199. Pitt D, Werner P, Raine CS. Glutamate excitotoxicity in a model of multiple sclerosis. Nat Med (2000) 6:67-70. doi:10.1038/71555
200. Smith T, Groom A, Zhu B, Turski L. Autoimmune encephalomyelitis ameliorated by AMPA antagonists. Nat Med (2000) 6:62-6. doi:10.1038/71548

201. Ikonomidou C, Turski L. Why did NMDA receptor antagonists fail clinical trials for stroke and traumatic brain injury? Lancet Neurol (2002) 1:383-6. doi:10.1016/S1474-4422(02)00164-3

202. Muir KW. Glutamate-based therapeutic approaches: clinical trials with NMDA antagonists. Curr Opin Pharmacol (2006) 6:53-60. doi:10.1016/j. coph.2005.12.002

203. Lovelace MD, Varney B, Sundaram G, Lennon MJ, Lim CK, Jacobs K, et al. Recent evidence for an expanded role of the kynurenine pathway of tryptophan metabolism in neurological diseases. Neuropharmacology (2016). doi:10.1016/j.neuropharm.2016.03.024

204. Haghikia A, Hohlfeld R, Gold R, Fugger L. Therapies for multiple sclerosis: translational achievements and outstanding needs. Trends Mol Med (2013) 19:309-19. doi:10.1016/j.molmed.2013.03.004

205. Hertenstein A, Schumacher T, Litzenburger U, Opitz CA, Falk CS, Serafini T, et al. Suppression of human CD4+ T cell activation by 3,4-dimethoxycinnamonyl-anthranilic acid (tranilast) is mediated by CXCL9 and CXCL10. Biochem Pharmacol (2011) 82:632-41. doi:10.1016/j.bcp.2011.06.013

206. Platten M, Ho PP, Youssef S, Fontoura P, Garren H, Hur EM, et al. Treatment of autoimmune neuroinflammation with a synthetic tryptophan metabolite. Science (2005) 310:850-5. doi:10.1126/science.1117634

207. Constantinescu SE, Constantinescu CS. Laquinimod (ABR-215062) for the treatment of relapsing multiple sclerosis. Expert Rev Clin Pharmacol (2016) 9:49-57. doi:10.1586/17512433.2016.1108189

208. Vollmer TL, Sorensen PS, Selmaj K, Zipp F, Havrdova E, Cohen JA, et al. A randomized placebo-controlled phase III trial of oral laquinimod for multiple sclerosis. J Neurol (2014) 261:773-83. doi:10.1007/s00415-014-7264-4

209. Abtin A, Jain R, Mitchell AJ, Roediger B, Brzoska AJ, Tikoo S, et al. Perivascular macrophages mediate neutrophil recruitment during bacterial skin infection. Nat Immunol (2014) 15:45-53. doi:10.1038/ni.2769

210. Roediger B, Kyle R, Yip KH, Sumaria N, Guy TV, Kim BS, et al. Cutaneous immunosurveillance and regulation of inflammation by group 2 innate lymphoid cells. Nat Immunol (2013) 14:564-73. doi:10.1038/ni.2584

211. Li JL, Goh CC, Keeble JL, Qin JS, Roediger B, Jain R, et al. Intravital multiphoton imaging of immune responses in the mouse ear skin. Nat Protoc (2012) 7:221-34. doi:10.1038/nprot.2011.438

212. Roediger B, Ng LG, Smith AL, Fazekas De St Groth B, Weninger W. Visualizing dendritic cell migration within the skin. Histochem Cell Biol (2008) 130:1131-46. doi:10.1007/s00418-008-0531-7

213. Germain RN, Miller MJ, Dustin ML, Nussenzweig MC. Dynamic imaging of the immune system: progress, pitfalls and promise. Nat Rev Immunol (2006) 6:497-507. doi:10.1038/nri1884

214. Padmanabhan K, Andrews SE, Fitzpatrick JA. Multi-photon imaging. Curr Protoc Cytom (2010) Chapter 2:Unit 2.9. doi:10.1002/0471142956.cy0209s54

215. Pai S, Qin J, Cavanagh L, Mitchell A, El-Assaad F, Jain R, et al. Real-time imaging reveals the dynamics of leukocyte behaviour during experimental cerebral malaria pathogenesis. PLoS Pathog (2014) 10:e1004236. doi:10.1371/ journal.ppat. 1004236

216. Pai S, Danne KJ, Qin J, Cavanagh LL, Smith A, Hickey MJ, et al. Visualizing leukocyte trafficking in the living brain with 2-photon intravital microscopy. Front Cell Neurosci (2012) 6:67. doi:10.3389/fncel.2012.00067

217. Cabrales P, Carvalho LJM. Intravital microscopy of the mouse brain microcirculation using a closed cranial window. J Vis Exp (2010) 45:e2184. doi: $10.3791 / 2184$

218. Holtmaat A, Bonhoeffer T, Chow DK, Chuckowree J, De Paola V, Hofer SB, et al. Long-term, high-resolution imaging in the mouse neocortex through a chronic cranial window. Nat Protoc (2009) 4:1128-44. doi:10.1038/nprot.2009.89

219. Fabene PF, Navarro Mora G, Martinello M, Rossi B, Merigo F, Ottoboni L, et al. A role for leukocyte-endothelial adhesion mechanisms in epilepsy. Nat Med (2008) 14:1377-83. doi:10.1038/nm.1878

220. Wilson EH, Weninger W, Hunter CA. Trafficking of immune cells in the central nervous system. J Clin Invest (2010) 120:1368-79. doi:10.1172/JCI41911

221. Mrass $\mathrm{P}$, Weninger W. Immune cell migration as a means to control immune privilege: lessons from the CNS and tumors. Immunol Rev (2006) 213:195-212. doi:10.1111/j.1600-065X.2006.00433.x

222. Harris TH, Banigan EJ, Christian DA, Konradt C, Tait Wojno ED, Norose K, et al. Generalized Levy walks and the role of chemokines in migration of effector CD8+ T cells. Nature (2012) 486:545-8. doi:10.1038/nature11098 
223. McGavern DB, Kang SS. Illuminating viral infections in the nervous system. Nat Rev Immunol (2011) 11:318-29. doi:10.1038/nri2971

224. Siffrin V, Brandt AU, Radbruch H, Herz J, Boldakowa N, Leuenberger T, et al. Differential immune cell dynamics in the CNS cause CD4+ T cell compartmentalization. Brain (2009) 132:1247-58. doi:10.1093/brain/awn354

225. Bartholomaus I, Kawakami N, Odoardi F, Schlager C, Miljkovic D, Ellwart JW, et al. Effector $\mathrm{T}$ cell interactions with meningeal vascular structures in nascent autoimmune CNS lesions. Nature (2009) 462:94-8. doi:10.1038/nature08478

226. Constantin G, Marconi S, Rossi B, Angiari S, Calderan L, Anghileri E, et al. Adipose-derived mesenchymal stem cells ameliorate chronic experimental autoimmune encephalomyelitis. Stem Cells (2009) 27:2624-35. doi:10.1002/ stem. 194

227. Pesic M, Bartholomaus I, Kyratsous NI, Heissmeyer V, Wekerle H, Kawakami N. 2-Photon imaging of phagocyte-mediated T cell activation in the CNS. J Clin Invest (2013) 123:1192-201. doi:10.1172/JCI67233

228. Siffrin V, Radbruch H, Glumm R, Niesner R, Paterka M, Herz J, et al. In vivo imaging of partially reversible th17 cell-induced neuronal dysfunction in the course of encephalomyelitis. Immunity (2010) 33:424-36. doi:10.1016/j. immuni.2010.08.018

229. Engelhardt B, Ransohoff RM. The ins and outs of T-lymphocyte trafficking to the CNS: anatomical sites and molecular mechanisms. Trends Immunol (2005) 26:485-95. doi:10.1016/j.it.2005.07.004

230. Ransohoff RM, Engelhardt B. The anatomical and cellular basis of immune surveillance in the central nervous system. Nat Rev Immunol (2012) 12:623-35. doi:10.1038/nri3265

231. Owens T, Bechmann I, Engelhardt B. Perivascular spaces and the two steps to neuroinflammation. J Neuropathol Exp Neurol (2008) 67:1113-21. doi:10.1097/NEN.0b013e31818f9ca8

232. Nayak D, Johnson KR, Heydari S, Roth TL, Zinselmeyer BH, McGavern DB. Type I interferon programs innate myeloid dynamics and gene expression in the virally infected nervous system. PLoS Pathog (2013) 9:e1003395. doi:10.1371/journal.ppat.1003395

233. Kawakami N, Nagerl UV, Odoardi F, Bonhoeffer T, Wekerle H, Flugel A. Live imaging of effector cell trafficking and autoantigen recognition within the unfolding autoimmune encephalomyelitis lesion. JExp Med (2005) 201:1805-14. doi:10.1084/jem.20050011

234. Davalos D, Ryu JK, Merlini M, Baeten KM, Le Moan N, Petersen MA, et al. Fibrinogen-induced perivascular microglial clustering is required for the development of axonal damage in neuroinflammation. Nat Commun (2012) 3:1227. doi: $10.1038 /$ ncomms 2230

235. O'Connor PW, Li D, Freedman MS, Bar-Or A, Rice GP, Confavreux C, et al. A phase II study of the safety and efficacy of teriflunomide in multiple sclerosis with relapses. Neurology (2006) 66:894-900. doi:10.1212/01. wnl.0000203121.04509.31

236. Chen Y, Guillemin G. The kynurenine pathway. In: Maurer M, editor. Amyotrophic Lateral Sclerosis, Chapter 15. InTech (2012). Available from: http://www.intechopen.com/books/amyotrophic-lateral-sclerosis/thekynurenine-pathway

Conflict of Interest Statement: The authors declare that the research was conducted in the absence of any commercial or financial relationships that could be construed as a potential conflict of interest.

Copyright ( 2016 Lovelace, Varney, Sundaram, Franco, Ng, Pai, Lim, Guillemin and Brew. This is an open-access article distributed under the terms of the Creative Commons Attribution License (CC BY). The use, distribution or reproduction in other forums is permitted, provided the original author(s) or licensor are credited and that the original publication in this journal is cited, in accordance with accepted academic practice. No use, distribution or reproduction is permitted which does not comply with these terms. 


\title{
Nutritional and Nanotechnological Modulators of Microglia
}

\author{
Dusica Maysinger* and Issan Zhang \\ Department of Pharmacology and Therapeutics, McGill University, Montreal, QC, Canada
}

Microglia are the essential responders to alimentary, pharmacological, and nanotechnological immunomodulators. These neural cells play multiple roles as surveyors, sculptors, and guardians of essential parts of complex neural circuitries. Microglia can play dual roles in the central nervous system; they can be deleterious and/or protective. The immunomodulatory effects of alimentary components, gut microbiota, and nanotechnological products have been investigated in microglia at the single-cell level and in vivo using intravital imaging approaches, and different biochemical assays. This review highlights some of the emerging questions and topics from studies involving alimentation, microbiota, nanotechnological products, and associated problems in this area of research. Some of the advantages and limitations of in vitro and in vivo models used to study the neuromodulatory effects of these factors, as well as the merits and pitfalls of intravital

\section{OPEN ACCESS}

Edited by:

Saparna Pai,

Centenary Institute, Australia

Reviewed by:

Ihsan Gursel,

Bilkent University, Turkey

Pavan Bhargava,

Johns Hopkins University

School of Medicine, USA

${ }^{*}$ Correspondence:

Dusica Maysinger

dusica.maysinger@mcgill.ca

Specialty section:

This article was submitted to

Multiple Sclerosis and

Neuroimmunology,

a section of the journal

Frontiers in Immunology

Received: 17 March 2016

Accepted: 27 June 2016

Published: 15 July 2016

Citation:

Maysinger D and Zhang I (2016)

Nutritional and Nanotechnological

Modulators of Microglia.

Front. Immunol. 7:270.

doi: 10.3389/fimmu.2016.00270 imaging modalities employed are presented.

Keywords: neuroinflammation, microglia, immunomodulation, nutrition, microbiota-gut-brain axis, intravital imaging, nanodelivery systems, nanomedicine

\section{INTRODUCTION}

Neuroinflammation has been considered a detrimental factor in many neurodegenerative diseases (e.g., Alzheimer's disease, Parkinson's disease, and multiple sclerosis) (1-4). As the resident immune cells of the brain, microglia play a central role in neuroinflammatory processes. Traditionally, microglia were considered seminal contributors to neurodegeneration associated with neuroinflammation $(5,6)$. However, this view is gradually changing (7). Under normal conditions, microglia survey the brain and perform essential housekeeping functions, ranging from the scavenging of cellular debris to synaptic remodeling, but they switch from "surveyors" to "attackers" or "protectors" when challenged by pathogens, injurious stimuli, or nanoparticulates (8-10). If excessively and chronically activated, microglia exert deleterious effects in the central nervous system (CNS) by secreting proinflammatory cytokines and interfering with synaptic integrity and functions (11, 12). Microglia exhibit at least four different functions: surveillance, phagocytosis, cytotoxicity, and neuroprotection. Depending on the nature and structure of the challenger, as well as the intensity, duration, and location of the challenge, activated microglia can take on a protective or destructive role (13). Signals from healthy and damaged neurons, astrocytes, and factors from the periphery also modulate the phenotype of activated microglia (14-16). Neuroprotection is achieved through different modes of their action, e.g., (i) synaptic stripping in development and motoneuron regeneration (5), (ii) promotion of neurogenesis in the injured CNS $(17,18)$, (iii) phagocytosis of misfolded proteins and damaged organelles $(19,20)$, and (iv) production of anti-inflammatory mediators, such as interleukin-4, interleukin-10, and transforming growth factor beta $(15,21-24)$. Cytokines, chemokines, neurotrophins, reactive oxygen species, and glutamate are endogenous signal molecules exchanged between neurons and glia cells (25-28) that can be modulated by 
pharmacological agents, but the access of these agents to the CNS may be limited by the blood-brain barrier $(29,30)$. More recently, it was shown that the microbiome can affect the integrity and function of the blood-brain barrier, as well as the maturation and phenotype of microglia (31-34). The emergence of drug nanocarriers and nanotechnological probes has facilitated the entry of therapeutics into the brain, but some of them exerted immunogenicity. The present review will focus on key neural factors and pharmacological targets in neuroinflammation, and discuss the potential of alimentary and nanotechnological agents in modulating immune processes in the brain. The merits and pitfalls of in vitro and in vivo models of neuroinflammation will be summarized, and the use of intravital imaging modalities to investigate neuroinflammation will be reviewed.

\section{MODULATORS OF NEUROINFLAMMATION}

\section{Alimentary and Environmental Neuroimmunomodulators}

Numerous genetic, environmental, and alimentary components can modulate neuroinflammation (35-37). For example, polluted urban air contains toxins, droplets, and particulates that are inhaled and travel though the blood stream, olfactory, and lymphatic systems to the brain, where they have been implicated in diseases of the CNS (37). Our daily diet can also affect neural cells, thereby altering their functions $(38,39)$. Rats fed high-fat diets were shown to have increased pro-inflammatory cytokines, such as tumor necrosis factor alpha (TNF- $\alpha$ ), interleukin-6 (IL-6), and interleukin-1 beta (IL-1 $\beta$ ), in the hypothalamus, compared to controls fed regular chow (40). The arcuate nucleus in the mediobasal hypothalamus is particularly sensitive to metabolic factors from the periphery, as it is located near the median eminence, a circumventricular organ unprotected by the blood-brain barrier (41). The activation of microglia in the arcuate nucleus of animals on high-fat diets, thus, demonstrates the direct impact of nutrition on neuroinflammation. Dietary components have also been used for therapeutic purposes as neuroprotective agents. For instance, vitamins E, C, and B have been shown to reduce oxidative stress in the brain $(42,43)$. The ketogenic diet is an established treatment for childhood epilepsy (44-46). It is thought that the increase in circulating polyunsaturated fatty acids can modulate ion channels, and that inflammation is altered by increasing circulating beta-hydroxybutyrate and activating hydroxy-carboxylic acid receptor 2 in immune cells (47-49). Ketogenic diets have since been proposed in neurological conditions, such as Alzheimer's disease and brain malignancies, but further clinical studies are required to confirm these findings and explain the beneficial effects at the molecular level (50-53). The polyunsaturated fatty acid docosahexaenoic acid (DHA) is a major component of neuronal cell membranes that is metabolized into resolvins and protectins, two families of neuroprotective lipid-derived mediators (54-56). Dietary DHA was shown to attenuate ischemic brain injury and pro-inflammatory markers in animal models (57-59). We have investigated the direct effects of DHA on synaptic integrity and indirect effects via microglia in the hippocampal CA1 region. Our studies have shown that DHA exerts neuroprotective effects in organotypic hippocampal tissue slices by preventing post synaptic spine deterioration (59). We also showed that DHA in microglia attenuates LPS-induced inflammation through the remodeling of lipid bodies and associated organelles (60). Furthermore, Bailey et al. provided evidence for the antioxidant role of lipid bodies in glia cells and neural stem cells $(61,62)$.

In addition to polyunsaturated fatty acids, such as DHA, numerous endogenous and exogenous fatty acids with different degree of saturation and chain lengths have been investigated in models of physiological and pathological conditions. The gut microbiota is an important source of small chain fatty acids (SCFA). Its population is heavily influenced by diet, and in turn, it modulates both the intestinal environment and overall human health (63-65). Once absorbed, SCFA directly impact on energy homeostasis in the liver, muscles, and adipose tissues, thereby affecting obesity and insulin resistance (66). SCFA can also affect the CNS by modulating neuroendocrine and cognitive responses, particularly when changes in the gut microbiome lead to increased intestinal permeability $(34,67,68)$. Emerging research on the gut-brain axis has shown that there is a tight link between the gut microbiota and the function of neural cells. The gut microbiota are necessary for the early and normal development of the brain, and contribute in programing the hypothalamic-pituitary-adrenal axis (69). In germ-free mice, microglia were found to have an immature phenotype, resulting in altered immune responses (31). Chronic enteric infections and antibiotics can also drastically modify the gut microbiome, resulting in neuropsychological symptoms $(34,70)$. The term "psychobiotics" has since been coined, referring to probiotics benefiting psychiatric illness, but further clinical studies are required to demonstrate the therapeutic benefits (71). While the composition and function of the gut microbiota can be affected by alimentary components, they can also be influenced by food contaminants, including nanoparticulate matter.

\section{Nanotechnological Immunomodulators}

Mammals have been exposed to airborne, waterborne, foodborne, and other nanomaterials in the environment for millennia and have developed mechanisms to deal with them $(72,73)$. Nevertheless, the explosion of nanoparticles in electronics, medical devices, paints, clothing, and cosmetics raised the awareness of the nanostructured materials in everyday life, requiring careful monitoring and analysis of the level and type of nanoparticles in soil, water, and air (74). In recent years, many nanomaterials have been designed for the development of diagnostics, delivery of therapeutic agents, and implants for the replacement of missing or impaired organ parts (e.g., joints, heart) (75-78). Some of these materials are well tolerated and efficiently eliminated, but others induce immune reactions and are toxic. Nanostructured materials are mainly recognized by cells of the immune system, primarily the mononuclear phagocytic system (MPS) (79). For example, internalized carbon nanotubes can be partly degraded in macrophages and the extent of biodegradation may be a major determinant in the severity of the associated inflammatory responses (80). Nanomaterial accumulation in macrophages 
within clearance organs (e.g., liver, kidneys, and spleen) can initiate both acute and chronic inflammation $(81,82)$. Although nanomaterials can cause toxic responses in these organs, technological manipulations of their morphologies, surfaces, sizes, charges, and porosities can minimize adverse effects (83-85). The structure-activity relationship of several classes of nanoparticles and outcome measures in immune and non-immune cells has been previously discussed $(86,87)$.

Our laboratory is particularly interested in investigating the effects of nanomaterials on microglia because of increasing evidence that (1) microglia are the major "consumers" of nanoparticles in the CNS $(10,88,89)$, (2) microglia and macrophages contribute to the maintenance and progression of glioblastoma, one of the most complex and deadly brain tumors (90), and (3) there is a structural and functional link between the CNS and lymphatic vessels (91). The discovery that lymphatic vessels lining dural sinuses are gateways between the systemic lymphatic system and the brain has recently re-defined our understanding of the immune system of the brain and is seminal in investigating neuroinflammatory and neurodegenerative disorders associated with impairments of the immune system. The majority of the studies showing either positive or negative effects of nanomaterials on the immune system focused on peripheral macrophages. This is understandable considering that most foreign materials are taken up by these cells. However, brain cancers, such as gliomas, are infiltrated mainly by the brain macrophages, the microglia. In fact, the proportion of microglia in low-grade gliomas can exceed $(>35 \%)$ the normal microglia contribution (10-15\%) in non-neoplastic brains. The majority of non-neoplastic cells in gliomas are tumorassociated macrophages (TAM) either originating from the periphery or intrinsic to the brain $(90,92)$. These cells form the microenvironment of the brain tumor and play a major role in the maintenance and progression of the cancer cells. They can contribute to cancer survival, invasiveness, and proliferation. Although the mechanisms underlying microglia stimulation of low- and high-grade gliomas are not fully understood, the existence of a unique tumor microenvironment resulting from the infiltration of central and peripheral macrophages provides an opportunity to establish more effective chemotherapeutic interventions (93). Achieving this goal is not simple because of the considerable diversity and plasticity of macrophages and microglia. The common classification of M1 polarization, deemed pro-inflammatory, and M2, designating alternatively activated macrophages (with subclasses M2a, M2b, and M2c), seems inadequate for TAM. RNA microarray analyses indicated that about 1000 transcripts were found to be differently expressed in glioblastoma-associated microglia and macrophages relative to control microglia. The expression patterns only partially $(<50 \%)$ overlapped with reported gene signatures for M1 and M2 macrophages (94). Therapeutic interventions targeting glioblastoma cells alone usually failed because of the contribution of the complex environment made of surrounding cells and brain tumor stem cells $(95,96)$. The problem is that macrophages and microglia secrete growth- and invasion-promoting factors, whereas brain tumor stem cells residing in perivascular niches often give raise to the resistance to radiation and chemotherapy
(97-100). By contrast, some data suggest that the ketogenic diet combined with standard cancer treatment could increase the sensitivity of cancer cells toward therapies due to their reliance on glycolytic metabolism (101). Such a diet could also decrease inflammation caused by infiltrating macrophages and microglia. Although the results are encouraging, additional clinical trials are required to confirm the previous findings, suggesting the beneficial effects of the ketogenic diet (102). Immunomodulation of the glioma microenvironment by nanoparticles is also an attractive therapeutic avenue to reduce tumor invasiveness and growth. Data from preclinical and clinical studies are encouraging despite limitations and hurdles, which need to be overcome before this strategy becomes more widely applied (103-105).

In inflammation, immunomodulation using nanoparticles could provide suitable alternatives to standard treatment strategies because of the versatility of particle surface modifications, compositions, and charges. Particles with a negative surface charge can bind to monocytes, marking them for sequestration by the spleen and preventing their migration and participation at the inflammation site (65). Interesting examples of polyanions with anti-inflammatory effects are dendritic polyglycerol sulfates (dPGS) (106-108). Studies with dPGS suggest that they are effective anti-inflammatory agents per se with strong inhibitory effects on inflammation-induced degenerative changes in microglia and the ability to rescue dendritic spine morphology (108). Their L-selectin binding in the low nanomolar range, limited impact on blood coagulation, and minor activation of the complement system render them attractive anti-inflammatory agents (106). A simplified molecular mechanism of dPGS binding to selectins and their intracellular location in microglia is illustrated (Figure 1).

Mechanisms for nanoparticle-induced tolerance and reduction of inflammation severity have been previously reviewed in Ref. (109). Although there are still numerous unanswered questions related to the mechanisms of nanoparticle-immune system interactions, it is anticipated that in the next decade, clinical studies will show if negatively charged biodegradable nanoparticles (e.g., polylactic-polyglycolic acid) will reduce severe inflammations in myocardial infarction and acute encephalitis syndrome. If these and similar studies show a positive outcome, nanoparticle-based therapies could become a valuable addition to existing therapies targeting the immune system (110). However, a series of safety testing and validation has to be performed in preclinical and clinical investigations. A tiered approach for assessing nanoparticle compatibility with the immune system in vitro during the early phase of preclinical development, strategies for designing early phase preclinical immunotoxicity screening, and challenges associated with these investigations have been reviewed in Ref. (86). Despite disappointments due to the lack of standards and standardized procedures, limited understanding of underlying mechanisms involved in nanoparticle-immune cell interactions, inadequate nanoparticle characterization and incomplete knowledge about plasma proteins and their interactions with nanoparticle surfaces under physiological and pathological conditions, results obtained so far have provided a baseline for investigations to harness biocompatible and safe nanomaterials for immunomodulation. 

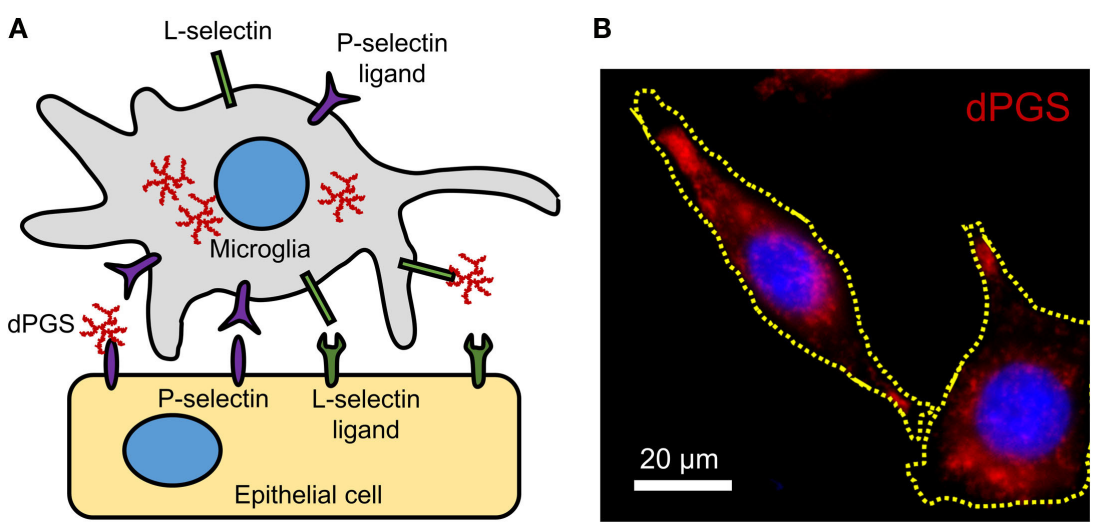

FIGURE 1 | (A) Molecular mechanism of dPGS binding to L-selectins and P-selectin ligands. (B) Fluorescence micrograph of fluorescently labeled dPGS (red) in microglia. Nuclei are labeled with Hoechst 33342 (blue).

\section{MODELS OF NEUROINFLAMMATION}

\section{In Vitro Models}

Neuroinflammation involves complex intercellular communication between different neural cell types organized into intricate networks. Thus, suitable primary neural cells in $3 \mathrm{D}$ cultures (prepared either from dissociated cells or organotypic slices) are preferable to cell line models grown in 2D (Figure 2). However, both types of in vitro models have important limitations (Table 1). Phenotypic traits of primary cells are often lost following in vitro culture, particularly in monolayer and monocultures. Microglia are ramified in the healthy brain and in astrocyte co-cultures, but in the absence of astrocytic support, they take on various morphologies (e.g., amoeboid, spindle, and rod like) (111). Astrocyte-conditioned media are only partly effective in maintaining the ramified morphology of microglia, because astrocytes provide not only soluble (e.g., granulocyte macrophage colonystimulating factor and colony-stimulating factor 1) but also nondiffusible factors. An astrocyte feeder layer is commonly used to support microglia and neuronal cultures alike $(112,113)$. This can be achieved using a two-chamber culture system comprising an enriched microglia culture separated from an enriched astrocyte culture by an inset with a porous membrane.

Immortalized microglia cell lines were initially established from rodents in the 1980s, and the first human cell line was reported in 1995 (114). N9 and BV2 are among the oldest and best-described murine microglia cell lines, while CHME and HMO6 are the main human microglia cell lines. Recently, another immortalized microglia cell line was generated from the adult murine brain (131). Beside the practical advantages of an established cell line, immortalized microglia provide a relatively homogeneous cell population that retains the phagocytic and secretory abilities of their primary counterparts. However, surface markers vary from cell line to cell line, and as with any continuous cell culture, phenotypic traits may change as cells differentiate over time (114). The systematic analysis of primary mouse and human microglia genes and microRNAs identified a unique molecular signature that was distinct from peripheral immune cells and immortalized microglia cell lines. This striking difference between primary and immortalized cells indicates that continuous cell lines are not always suitable to answer some questions, such as the role of surface markers highly expressed in human or mouse microglia [e.g., purinergic receptor P2Y, G-protein coupled, 12 (P2ry12) in human, and Fc receptor-like S (FCRLS) in mouse microglia] $(132,133)$.

Brain slices are 3D, ex vivo models with partial brain architecture and synaptic circuitries. These models are used to investigate intercellular communication between neural cells under "physiological" and pathological conditions. Organotypic brain cultures are usually prepared from postnatal animals (days 3-9), and slices are maintained in culture until the maturation of the synaptic networks. Although the structural development of organotypic brain slices has been found to be largely comparable to that of age-matched animals, it has been reported that these ex vivo cultures had increased dendrite numbers and glutamatergic synaptic currents resulting from the rewiring of axons damaged during the initial slice preparation (120). Nevertheless, the preservation of tissue structure and the presence of microglia in organotypic brain slices are major advantages in the study of neuroinflammation. Acute brain slices are similar to organotypic brain cultures. They can be harvested from animals of any age, and experiments are typically completed within hours. However, the biomechanical stress caused by tissue slicing, presence of damaged cells, and release of soluble factors from these cells must be considered when interpreting results from such preparations (134).

Inflammation in neural cells can be induced using pathogenderived ligands, pro-inflammatory cytokines, and injurious stimuli. Among the most common pro-inflammatory stimuli is lipopolysaccharide (LPS), an endotoxin from Gram-negative bacteria, which binds to toll-like receptor 4 (TLR4) on microglia, astrocytes, oligodendrocytes, and neurons $(14,135)$. The production of cytokines (e.g., interleukin-1 beta, interleukin-6, interleukin-18, interleukin-33) by microglia in response to LPS is mediated by the inflammasome, a multiprotein complex typically composed of pro-caspase-1, the adaptor molecule apoptosisassociated speck-like protein containing a caspase recruitment 


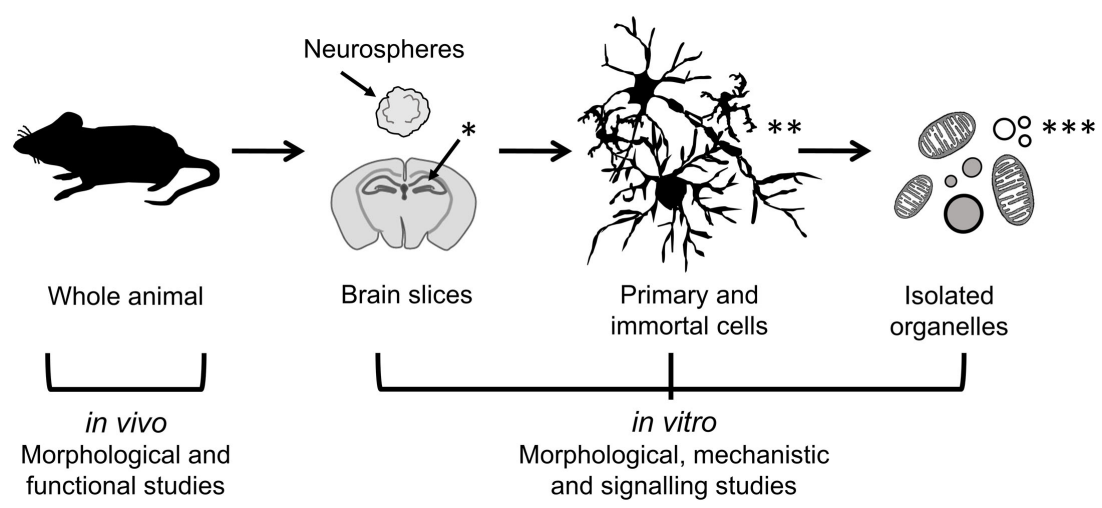

FIGURE 2 | Models of different complexity used to study the effects of immunomodulators in neural cells. In vivo models of neuroinflammation are most suitable for morphological and functional studies, while in vitro models of neural cells in 2D (primary and immortal dissociated cells) and 3D (neurospheres and brain slice cultures) are useful for morphological, mechanistic, and signaling studies. Isolated organelles can be used to investigate mechanisms of inflammation at the subcellular level. [ ${ }^{*}$ Hippocampus (hippos = horse; campos = sea monster); ${ }^{* *}$ neurons, microglia, astrocytes; ${ }^{* \star *}$ organelles: mitochondria, lipid droplets, lysosomes, nucleoli.]

domain (ASC) and nucleotide-binding oligomerization domain, leucine-rich repeat-containing receptor (NLR) family proteins (Figure 3) (136). Different types of inflammasomes can assemble depending on the nature and intensity of the stimulus, and many members of the NLR family can facilitate the assembly (e.g., NLRP1, NLRP7, and NLRP12). In particular, the NLR family, pyrin domain-containing 3 (NLRP3) inflammasome is common in neuroinflammation-associated disorders, and can be regulated by a wide variety of factors, such as pathogen-associated molecular patterns, damage-associated molecular patterns, COX-2 activity, and damaged mitochondria (137-139). In Alzheimer's disease, traumatic brain injuries (TBI), and MS, the NLRP3 inflammasome was found to exacerbate inflammatory responses and damage mediated by microglia (140-143). Notably, hyperactivation of microglia characterized by inflammasome activation and cytokine release can lead to the programed cell death pyroptosis in neural cells (144-146). Pro-inflammatory cytokines are major inducers of immune activation, both in the peripheral and central immune systems. These include, among others, TNF- $\alpha$, IFN $\gamma$, IL-1 $\beta$, and IL-6 $(26,147,148)$. Modulation of IL- 6 classical and trans-signaling has been exploited for therapeutic interventions in several preclinical and clinical trials $(149,150)$. The evolutionary conserved glycoprotein 130 (gp130) system inspired the development of sgp130Fc, an effective pharmacological tool to distinguish classical from trans-signaling. The results from phase III studies with sgp130Fc are awaited - it is anticipated that blockade of trans-signaling will prove to be superior to the global blockade of IL-6 signaling by the neutralizing antibody tocilizumab. Recent studies showed that the small molecule LMT-28 can also block trans-signaling of IL-6 (151). LMT-28 is stable, simple to synthesize, and functions by binding directly to gp 130 . Clinical data for its effectiveness in neurological disorders are not yet available. Anti-inflammatory cytokines, such as interleukin-4 and -10 , can dampen the effects of pro-inflammatory stimuli. The production of these secreted factors can be monitored using enzyme-linked immunosorbent assays (ELISA). Inflammation induced by ischemic and TBI is difficult to replicate in vitro, but some morphological and biochemical changes can be assessed in simplified models. For instance, oxygen and glucose deprivation (OGD) is often used to mimic brain ischemia and induce the activation of toll-like receptors 2 and 4 in primary cortical neurons (135). Transection, compression, hydrostatic pressure, and stretch injuries are other examples of brain "injuries in the dish" (134).

\section{In Vivo Models}

A great number of animal models of neuroinflammation are available today, many of which are disease specific (see examples in Table 1). Although transgenic animals are popular to examine the effects of gene knock-in and knock-out, wild-type animals remain necessary to understand the fundamental pathophysiology of neuroinflammation. LPS can be injected either systemically or intracranially. Circulating LPS rapidly causes an inflammatory response in the brain, first at the circumventricular organs, then across the CNS (152). Although the choice of LPS serotype has little impact on TLR4 stimulation, it can significantly affect in vivo studies involving the adaptive immune system. The degree of purity of the LPS is also an important factor, as products of lesser quality can contain other pathogen-associated molecules that will alter the potency of the LPS and the magnitude of the inflammatory response. Systemic injection is often administered intraperitoneally, intravenously, or by stereotaxic administration directly into the brain parenchyma. The stereotaxic apparatus holds the head of the animal in place and a stereotaxic atlas is used to determine the coordinates for the site at which a small hole in the skull should be drilled to access a specific site in the brain (153). Until recently, innate recognition of LPS was limited to its membrane receptor TLR4/MD-2-stimulated cytokine transcription. Therapeutic intervention by Eritoran has achieved very moderate success in sepsis (154). This could be in part because of the existence of non-canonical LPS signaling induced by cytosolic LPS. This non-canonical signaling via intracellular 
TABLE 1 | Models of immunomodulation: from in vitro to in vivo models.

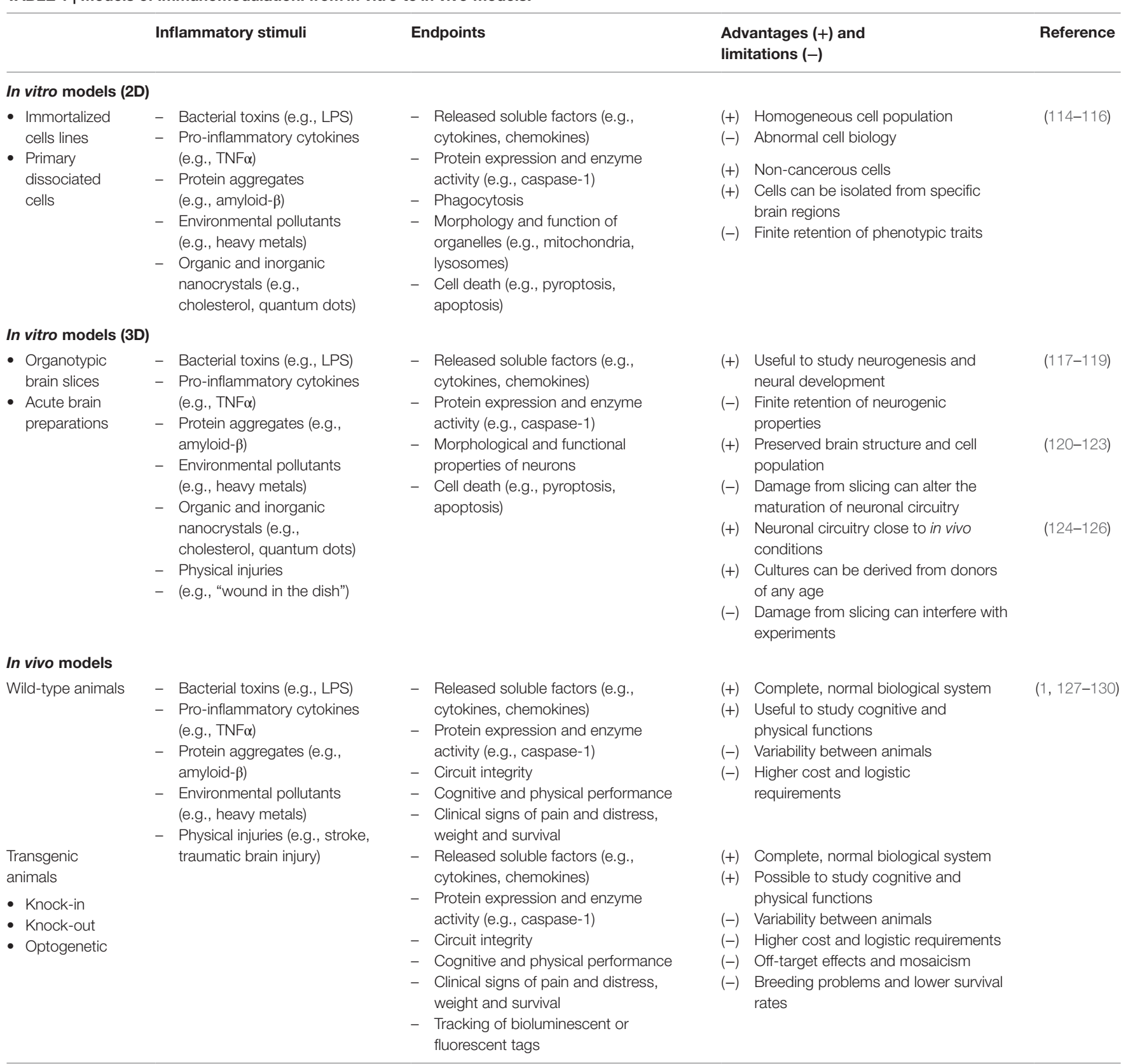

LPS activates pro-inflammatory caspases - caspase-11 in mice and caspase-4/5 in humans - and does not depend on TLR4 (155-157). LPS binding to caspases induces their oligomerization, which is a prerequisite for caspase activation. A simplified model of canonical and non-canonical signaling by LPS is illustrated (Figure 4). Resulting CNS complications, such as encephalopathy, are mainly mediated by neuroinflammation and oxidative stress (158). Aside from LPS administration, inflammation can be induced more globally by bacterial infections. A standard method to induce polymicrobial sepsis is cecal ligation (159). It is easily performed, and the severity of the disease can be controlled to a certain extent (160). However, there is a high mortality rate, and variable outcomes have been observed between animals and laboratories (161).

Inflammatory processes in transgenic models of neuroinflammation often result indirectly from the expression of a diseasespecific mutant gene, and most models were developed for the study of neurodegenerative diseases. The APP/PS1 mouse model, for instance, is used in the study of Alzheimer's disease. These mice express a chimeric amyloid precursor protein and a mutant human presenilin-1, causing the accumulation of amyloid-beta plaques by the age of 6 months, extensive neuroinflammation and, later on, memory impairment (162). By contrast, it was recently suggested that the amyloid beta peptide can protect 


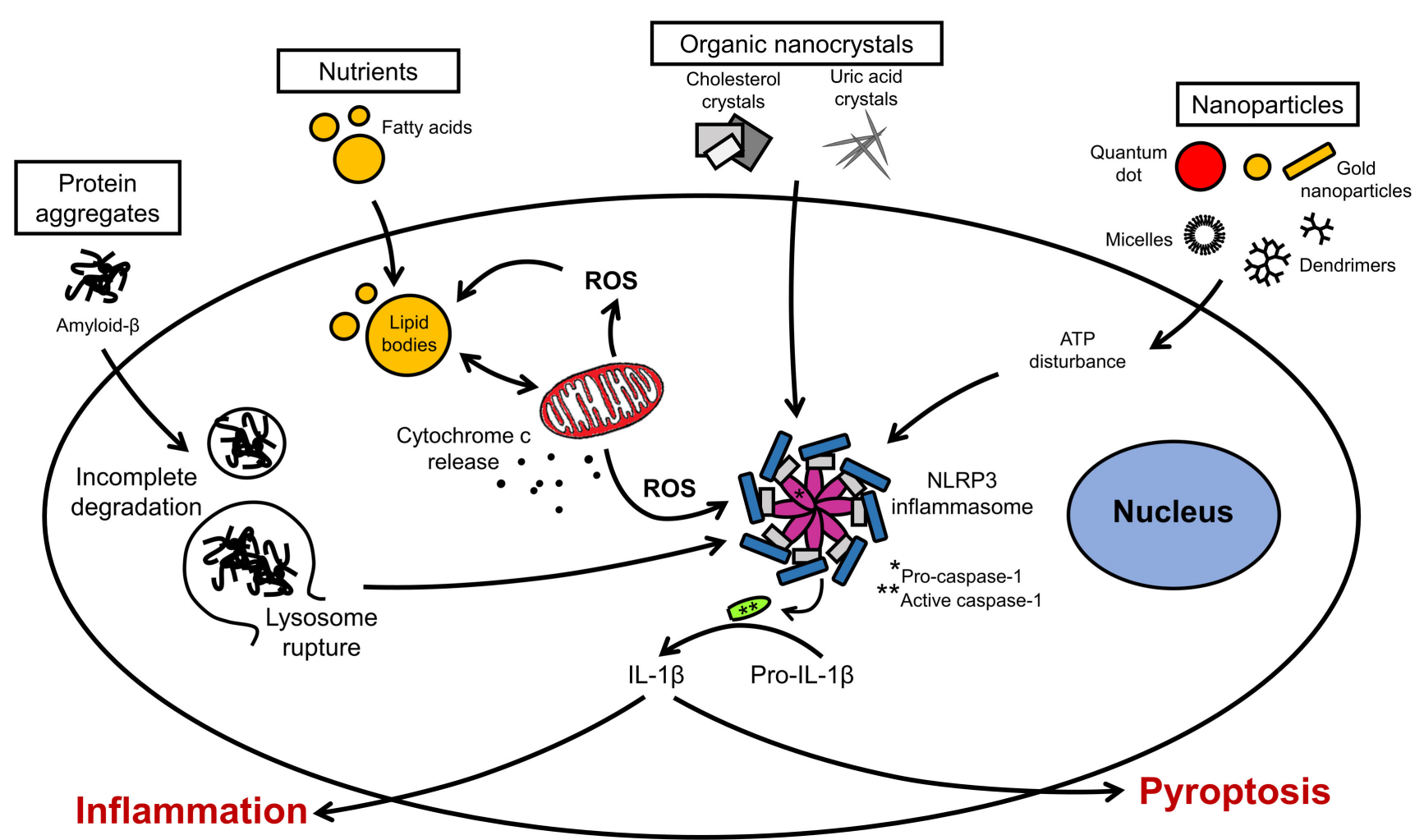

FIGURE 3 | Organellar remodeling in inflammation. Multiple pro-inflammatory stimuli can disrupt redox homeostasis in microglia. Mitochondria are the major source of reactive oxygen species (ROS). Excessive ROS induces the formation of lipid bodies and impairs their communication with intracellular organelles. Several signal transduction pathways implicated in inflammation converge on the inflammasome. Inflammasome activation leads to the caspase activation and cytokine release. Modulation of these pathways can lead to resolution of inflammation or exacerbation with pyroptotic cell death.

against microbial infection in a mouse model of Alzheimer's disease (163). This is an intriguing proposition, raising the possibility that amyloid beta may play a protective role in innate immunity through its binding to microbial cell walls via heparinbinding domains. In the adeno-associated virus-alpha-synuclein mouse model of Parkinson's disease, the animal expresses alphasynuclein under the control of a viral promoter. This results in the loss of dopaminergic neurons, as well as the activation of microglia (164). For the study of amyotrophic lateral sclerosis, transgenic mice expressing a mutant superoxide dismutase 1 gene were observed to show astrocyte and microglia activation, leading to motoneuron degeneration and muscle atrophy $(165,166)$. Transgenic mouse models used to investigate neuroinflammation can provide valuable information on morphological, biochemical, and functional changes in neural cells, but they have limitations that must be considered in the context of human pathology (1). Other knock-out and knock-in animals have also been employed to study the role of key mediators of neuroinflammation. Caspase-1 knock-out mice, for example, seemed more resistant to ischemia-induced neural cell death than wild-type animals (167). More recently, the clustered regularly interspaced short palindromic repeats (CRISPR) and CRISPR-associated protein- 9 (Cas9) gene editing technique has generated considerable excitement, as it was successful in targeting single or multiple genes in the mouse brain (168). The technique allows the generation of mutant animals with ease and efficiency compared to the traditional transfection of mouse embryonic stem cells. However, emerging problems include off-site effects and mosaicism (169).

\section{INTRAVITAL IMAGING OF NEUROINFLAMMATION}

A great variety of reporters and probes are currently available to investigate neuroinflammation at the cellular level (170-173). Cellular events of interest include the migration and phagocytic activity of microglia, the infiltration of peripheral immune cells, as well as the production of secreted factors, metabolism, and viability of neural cells. Intravital imaging is useful to study the pathophysiology of neuroinflammation in a non-invasive manner, but an important limitation is the scattering and absorbance of light entering biological tissues. The availability of strong reporters and powerful imaging modalities have allowed for better detection and facilitated the generation of quantitative data from investigated signals while minimizing autofluorescence. The natural fluorescence of different tissues can mask signals from fluorescent probes. Lipofuscin, which can be excited anywhere in the range of 360-647 nm, is commonly found in neurons and glia cells, and increases with animal age. The imaging of green fluorescent protein, one of the most common and popular fluorescent labels, can also be hindered by a subset of green autofluorescent cells 


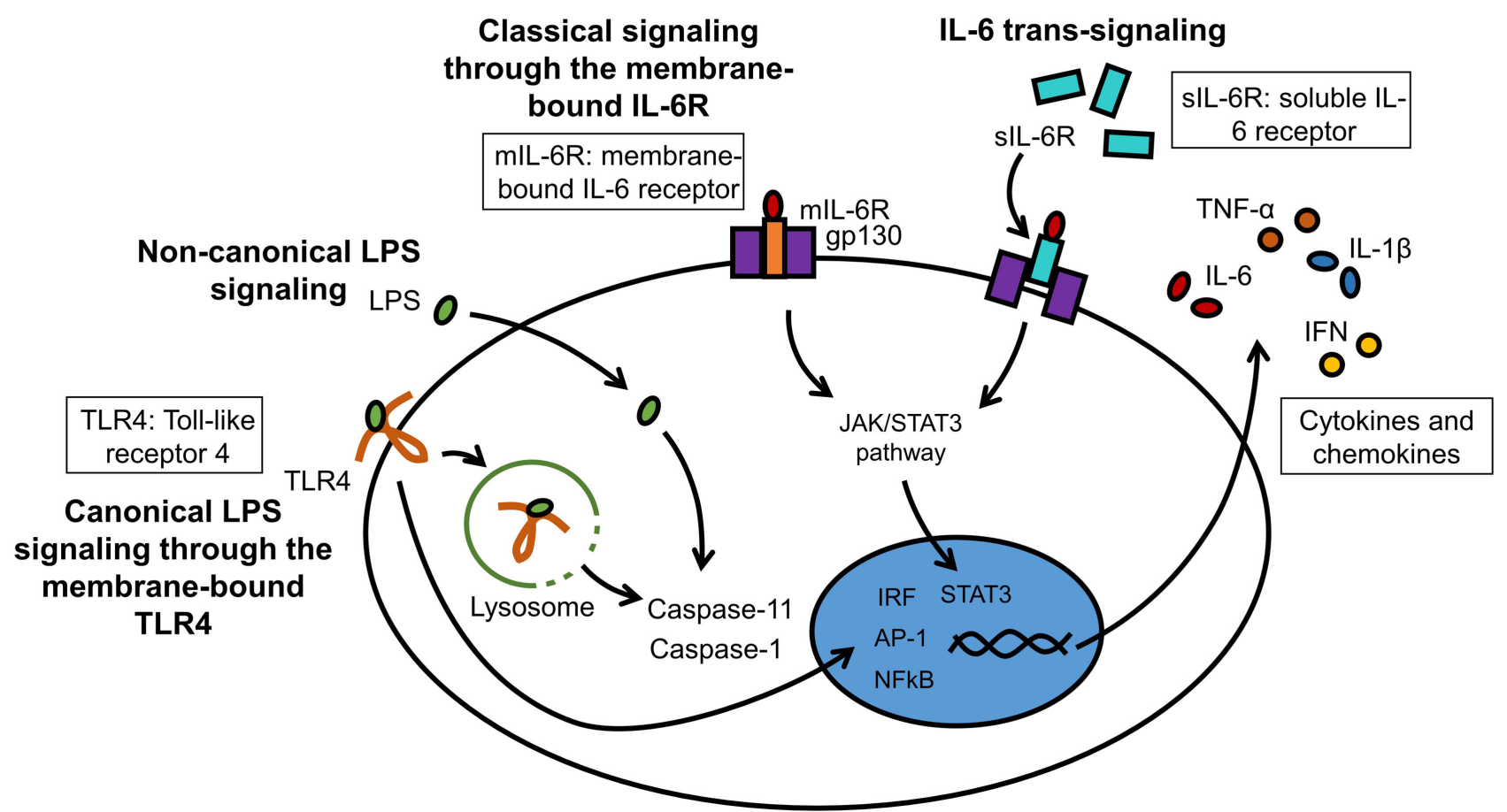

FIGURE 4 | LPS and IL-6 signaling in microglia. LPS can interact with membrane-bound TLR4 (canonical signaling) or can enter the cytosol independently from TLR4 (non-canonical signaling). The major cytosolic receptors for LPS are pro-inflammatory caspases. IL-6 binds either to the membrane receptor IL-6R (mIL-6R; classical signaling) or to the soluble IL-6 receptor (SIL-6R; trans-signaling). These receptor complexes subsequently bind to gp130 to initiate intracellular signaling cascades.

in the rat cortex and hippocampus (174). In tissue sections, the risk of false positives can be reduced by using autofluorescence quenchers, such as copper sulfate (175). The choice of fluorophores emitting in the near-infrared spectrum can be made to avoid this issue. Imaging of structural and functional changes in the living brain can be performed using open-skull preparations, where a small window in the skull is protected by a glass coverslip. Following the implantation of the cranial window, a recovery time is necessary to avoid inflammation caused by the surgery $(176,177)$. However, long-term imaging using the open-skull technique can be obscured by bone re-growth and the thickening of the meninges (178). Imaging of the cortex using the thinnedskull cranial window technique is useful when longer intervals are needed in between imaging sessions. However, repeated imaging requires the re-thinning of the skull, which has to be carefully monitored to avoid cortical trauma and inflammation $(179,180)$. For both imaging techniques, two-photon microscopy in the near infrared region is suitable to avoid photobleaching and photodamage.

Transgenic animals expressing luciferase in glia cells have been employed to track and image processes in neuroinflammation at the cellular level (181-183). Our studies have shown marked activation of microglia, pro-inflammatory caspases, and astrocytes by nanocrystals (184-186). Data from these studies showed that stable nanocrystals injected directly into the parenchyma of mice induced transient astrocyte activation, suggesting that only nanocrystals adequately coated with polyethylene glycol (PEG) are suitable nanotechnological tools. Glia cells were also activated by gold nanoparticles, depending on the nanoparticles' morphology (10). Activation of glia cells is often accompanied by the activation of inflammatory caspases and caspases implicated in apoptosis (187). Nanosensors for caspases have been developed, and examples of constructs for these sensors are illustrated in Figure 5 (170, 188).

A whole palette of fluorescent proteins, mostly mutant derivatives of the jellyfish's green fluorescent protein, have also been employed to "illuminate" the brain. The use of cell-typespecific fluorescent labels allowed to map brain structures and to distinguish different cell populations with greater accuracy. High-resolution pictures have been recorded in recent years, and unprecedented 3D images and videos have been produced from fluorescently labeled brain tissues $(189,190)$. Although the quality of these imaging techniques remains variable and is dependent on the success of the genetic probes and the available imaging modalities, these techniques have been instrumental in understanding structural and functional aspects of the CNS - including glia-neuron interactions. Optogenetics have also been used to study light-responsive channels and other proteins in neural cells (191-193). For instance, the selective expression of channelrhodopsin-2, a light-responsive membrane channel, has been employed to study calcium signaling in astrocytes in vitro and in vivo (194). Optogenetic tools could, thus, be used to reveal the contribution of microglia in neuroinflammatory processes (195). Although optogenetics has generated valuable information on macromolecules in cells, this approach cannot be applied to investigate small molecules, such as phospholipids. More 
A

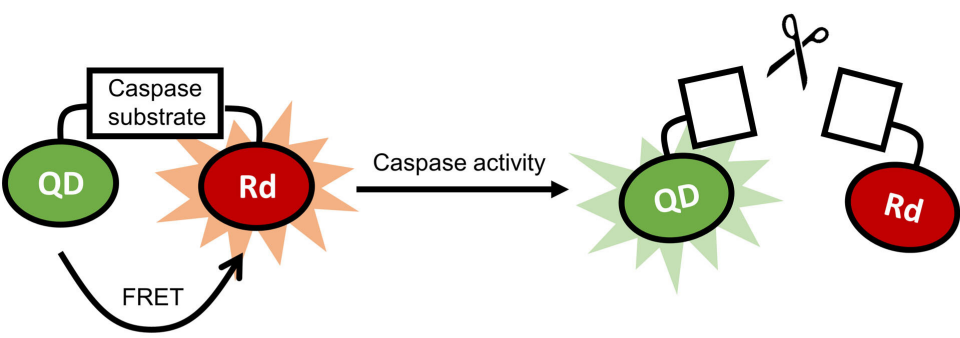

B
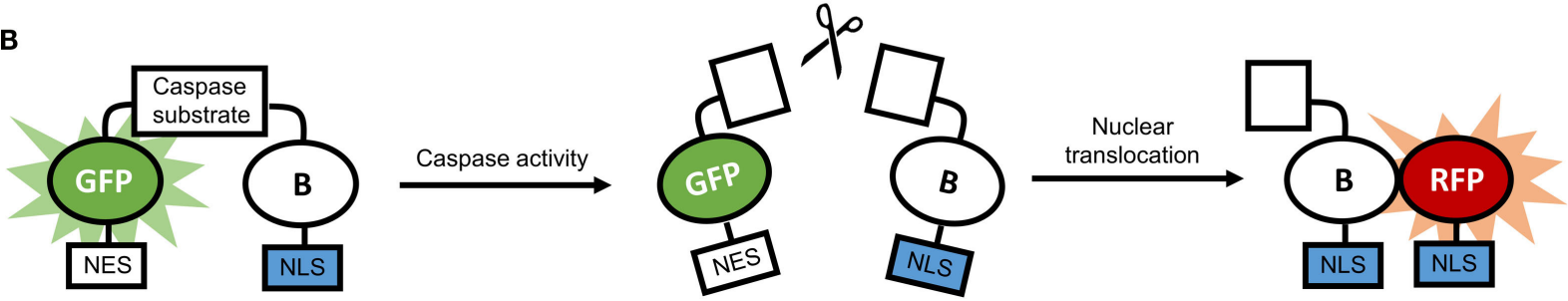

FIGURE 5 | (A) Schematic representation of a quantum dot-based sensor for caspase activity. In the absence of caspase activity, there is fluorescence resonance energy transfer (FRET) between the quantum dot (QD) and the rhodamine molecule (Rd), and the fluorescence of the QD is quenched. In the presence of caspase activity, FRET is disrupted, and the QD is fluorescent. (B) Schematic representation of a ratiometric biosensor for caspase activity. In the absence of caspase activity, the dimerization-dependent green fluorescent protein (GFP) is dimerized with the partner protein B and is retained in the cytoplasm through a nuclear exclusion signal (NES). In the presence of caspase activity, the dimerization is disrupted, and B translocated to the nucleus using a nuclear localization signal (NLS), and associates with the dimerization-dependent red fluorescent protein (RFP). As a result, green fluorescence in the cytoplasm fades, and red fluorescence in the nucleus increases.

recently, the approach of optolipidomics was used to study the processing of mitochondria-specific cardiolipins in apoptosis (196). Mitochondrial functions are often impaired in inflammatory processes, and the combination of optogenetics and optolipidomics could provide complementary information on underlying intricacies in neuroinflammation.

\section{CONCLUSION}

Neuroinflammation is considered a significant contributor in many neurodegenerative diseases. Microglia are the immune cells of the CNS, and are modulated by numerous factors, including alimentary products and the gut microbiome. Nanoparticulates have emerged as a new group of "xenobiotics" that must be thoroughly characterized prior to investigating their immunomodulatory effects in the CNS and elsewhere. Nanotechnology offers a wide selection of shape- and size-tunable probes, ranging from quantum dots to fluorescently labeled polymeric constructs (163). Nanoprobes can be brighter and more stable than genetic probes, and designed to "activate" in response to a particular stimuli, such as light or acidic $\mathrm{pH}$. However, nanotechnological probes are often large and cannot reach desirable intracellular locations. In addition, these probes are complex and relatively little is known about their stability in vivo, as well as their pharmacokinetics and pharmacodynamics $(186,187)$. It is well established that the biological identity of a nanoparticle is distinct from its well-defined chemical identity. Serum protein binding, sensitivity to $\mathrm{pH}$, and clearance rates are all factors affecting the immunogenicity and fate of a nanoparticle in vivo $(146,147)$. On the other hand, nanoparticle-induced immune responses can be exploited for improving vaccine efficiency and boost the immune system in pathologies with weakened immune responsiveness (197-199). Diverse fluorescent nanostructures can provide tools for the tracking and imaging of complex networks in different cell types in a spatio-temporal manner. The combined use of nanotechnological tools and advanced intravital imaging techniques can, thus, provide unprecedented insight into the mechanisms of neuroinflammation. Exciting data related to brain abnormalities implicating glial cells come from gene editing techniques, such as CRISPR/Cas9 (168). Animal studies exploiting these approaches in mice models of neurodegenerative diseases will help to reveal intricacies in neural circuitries under physiological conditions and mechanisms involved in multifactorial diseases associated with neuroinflammation.

\section{AUTHOR CONTRIBUTIONS}

DM outlined, co-wrote, and revised the manuscript. IZ drafted and finalized the figures and table, and co-authored the text. Both authors read and approved of the final version of the manuscript.

\section{ACKNOWLEDGMENTS}

The authors thank Mr. Jeff Ji for reading the manuscript. This work was supported by grants from the Natural Sciences and Engineering Council of Canada (RGPIN-2015-04994) and the Canadian Institutes of Health Research (119425). 


\section{REFERENCES}

1. Heneka MT, Kummer MP, Latz E. Innate immune activation in neurodegenerative disease. Nat Rev Immunol (2014) 14:463-77. doi:10.1038/ nri3705

2. Hunot S, Hirsch EC. Neuroinflammatory processes in Parkinson's disease. Ann Neurol (2003) 53(Suppl 3):S49-58. doi:10.1002/ana.10481

3. Nolan YM, Sullivan AM, Toulouse A. Parkinson's disease in the nuclear age of neuroinflammation. Trends Mol Med (2013) 19:187-96. doi:10.1016/j. molmed.2012.12.003

4. Whalley K. Neuroinflammation: transport disruption in multiple sclerosis. Nat Rev Neurosci (2015) 16:2-2. doi:10.1038/nrn3892

5. Kettenmann H, Kirchhoff F, Verkhratsky A. Microglia: new roles for the synaptic stripper. Neuron (2013) 77:10-8. doi:10.1016/j.neuron.2012. 12.023

6. Perry VH, Nicoll JAR, Holmes C. Microglia in neurodegenerative disease. Nat Rev Neurol (2010) 6:193-201. doi:10.1038/nrneurol.2010.17

7. Chen Z, Trapp BD. Microglia and neuroprotection. J Neurochem (2016) 136(Suppl 1):10-7. doi:10.1111/jnc.13062

8. Sierra A, Beccari S, Diaz-Aparicio I, Encinas JM, Comeau S, Tremblay M-È. Surveillance, phagocytosis, and inflammation: how never-resting microglia influence adult hippocampal neurogenesis. Neural Plast (2014) 2014:610343. doi:10.1155/2014/610343

9. Kreutzberg GW. Microglia: a sensor for pathological events in the CNS. Trends Neurosci (1996) 19:312-8. doi:10.1016/0166-2236(96)10049-7

10. Hutter E, Boridy S, Labrecque S, Lalancette-Hébert M, Kriz J, Winnik FM, et al. Microglial response to gold nanoparticles. ACS Nano (2010) 4:2595-606. doi:10.1021/nn901869f

11. Block ML, Zecca L, Hong J-S. Microglia-mediated neurotoxicity: uncovering the molecular mechanisms. Nat Rev Neurosci (2007) 8:57-69. doi:10.1038/ nrn2038

12. Hanisch U-K. Microglia as a source and target of cytokines. Glia (2002) 40:140-55. doi:10.1002/glia.10161

13. Hanisch U-K, Kettenmann H. Microglia: active sensor and versatile effector cells in the normal and pathologic brain. Nat Neurosci (2007) 10:1387-94. doi:10.1038/nn1997

14. Lehnardt S. Innate immunity and neuroinflammation in the CNS: the role of microglia in Toll-like receptor-mediated neuronal injury. Glia (2010) 58:253-63. doi:10.1002/glia.20928

15. Cherry JD, Olschowka JA, O'Banion MK. Neuroinflammation and M2 microglia: the good, the bad, and the inflamed. J Neuroinflammation (2014) 11:98. doi:10.1186/1742-2094-11-98

16. Savarin C, Hinton DR, Valentin-Torres A, Chen Z, Trapp BD, Bergmann CC, et al. Astrocyte response to IFN- $\gamma$ limits IL-6-mediated microglia activation and progressive autoimmune encephalomyelitis. J Neuroinflammation (2015) 12:79. doi:10.1186/s12974-015-0293-9

17. Ernst A, Alkass K, Bernard S, Salehpour M, Perl S, Tisdale J, et al. Neurogenesis in the striatum of the adult human brain. Cell (2014) 156:1072-83. doi:10.1016/j.cell.2014.01.044

18. Nikolakopoulou AM, Dutta R, Chen Z, Miller RH, Trapp BD. Activated microglia enhance neurogenesis via trypsinogen secretion. Proc Natl Acad Sci U S A (2013) 110:8714-9. doi:10.1073/pnas.1218856110

19. Sierra A, Abiega O, Shahraz A, Neumann H. Janus-faced microglia: beneficial and detrimental consequences of microglial phagocytosis. Front Cell Neurosci (2013) 7:6. doi:10.3389/fncel.2013.00006

20. Brown GC, Neher JJ. Microglial phagocytosis of live neurons. Nat Rev Neurosci (2014) 15:209-16. doi:10.1038/nrn3710

21. Lindholm D, Castrén E, Kiefer R, Zafra F, Thoenen H. Transforming growth factor-beta 1 in the rat brain: increase after injury and inhibition of astrocyte proliferation. J Cell Biol (1992) 117:395-400. doi:10.1083/jcb. 117.2.395

22. Liu X, Liu J, Zhao S, Zhang H, Cai W, Cai M, et al. Interleukin-4 is essential for microglia/macrophage M2 polarization and long-term recovery after cerebral ischemia. Stroke (2016) 47:498-504. doi:10.1161/STROKEAHA. 115.012079

23. Park KW, Lee HG, Jin BK, Lee YB. Interleukin-10 endogenously expressed in microglia prevents lipopolysaccharide-induced neurodegeneration in the rat cerebral cortex in vivo. Exp Mol Med (2007) 39:812-9. doi:10.1038/ emm. 2007.88
24. Ledeboer A, Brevé JJ, Poole S, Tilders FJ, Van Dam AM. Interleukin-10, interleukin-4, and transforming growth factor-beta differentially regulate lipopolysaccharide-induced production of pro-inflammatory cytokines and nitric oxide in co-cultures of rat astroglial and microglial cells. Glia (2000) 30:134-42. doi:10.1002/(SICI)1098-1136(200004)30:2<134::AID-GLIA3> 3.0.CO;2-3

25. Fields RD, Stevens-Graham B. New insights into neuron-glia communication. Science (2002) 298:556-62. doi:10.1126/science.298.5593.556

26. Choi SS, Lee HJ, Lim I, Satoh J, Kim SU. Human astrocytes: secretome profiles of cytokines and chemokines. PLoS One (2014) 9:e92325. doi:10.1371/ journal.pone.0092325

27. Elkabes S, DiCicco-Bloom EM, Black IB. Brain microglia/macrophages express neurotrophins that selectively regulate microglial proliferation and function. J Neurosci (1996) 16:2508-21.

28. Domercq M, Vazquez N, Matute C. Neurotransmitter signaling in the pathophysiology of microglia. Front Cell Neurosci (2013) 7:49. doi:10.3389/ fncel.2013.00049

29. Palmer AM. Multiple sclerosis and the blood-central nervous system barrier. Cardiovasc Psychiatry Neurol (2013) 2013:530356. doi:10.1155/ 2013/530356

30. Erickson MA, Dohi K, Banks WA. Neuroinflammation: a common pathway in CNS diseases as mediated at the blood-brain barrier. Neuroimmunomodulation (2012) 19:121-30. doi:10.1159/000330247

31. Erny D, Hrabě de Angelis AL, Jaitin D, Wieghofer P, Staszewski O, David E, et al. Host microbiota constantly control maturation and function of microglia in the CNS. Nat Neurosci (2015) 18:965-77. doi:10.1038/nn.4030

32. Sherwin E, Rea K, Dinan TG, Cryan JF. A gut (microbiome) feeling about the brain. Curr Opin Gastroenterol (2016) 32:96-102. doi:10.1097/ MOG.0000000000000244

33. Burokas A, Moloney RD, Dinan TG, Cryan JF. Microbiota regulation of the mammalian gut-brain axis. Adv Appl Microbiol (2015) 91:1-62. doi:10.1016/ bs.aambs.2015.02.001

34. Kelly JR, Kennedy PJ, Cryan JF, Dinan TG, Clarke G, Hyland NP. Breaking down the barriers: the gut microbiome, intestinal permeability and stressrelated psychiatric disorders. Front Cell Neurosci (2015) 9:392. doi:10.3389/ fncel.2015.00392

35. Malik M, Parikh I, Vasquez JB, Smith C, Tai L, Bu G, et al. Genetics ignite focus on microglial inflammation in Alzheimer's disease. Mol Neurodegener (2015) 10:52. doi:10.1186/s13024-015-0048-1

36. Cai D. Neuroinflammation and neurodegeneration in overnutritioninduced diseases. Trends Endocrinol Metab (2013) 24:40-7. doi:10.1016/j. tem.2012.11.003

37. Block ML, Calderón-Garcidueñas L. Air pollution: mechanisms of neuroinflammation and CNS disease. Trends Neurosci (2009) 32:506-16. doi:10.1016/j.tins.2009.05.009

38. Timmermans S, Bogie JFJ, Vanmierlo T, Lütjohann D, Stinissen P, Hellings $\mathrm{N}$, et al. High fat diet exacerbates neuroinflammation in an animal model of multiple sclerosis by activation of the renin angiotensin system. J Neuroimmune Pharmacol (2014) 9:209-17. doi:10.1007/ s11481-013-9502-4

39. Castanon N, Luheshi G, Layé S. Role of neuroinflammation in the emotional and cognitive alterations displayed by animal models of obesity. Front Neurosci (2015) 9:229. doi:10.3389/fnins.2015.00229

40. De Souza CT, Araujo EP, Bordin S, Ashimine R, Zollner RL, Boschero AC, et al. Consumption of a fat-rich diet activates a proinflammatory response and induces insulin resistance in the hypothalamus. Endocrinology (2005) 146:4192-9. doi:10.1210/en.2004-1520

41. Valdearcos M, Xu AW, Koliwad SK. Hypothalamic inflammation in the control of metabolic function. Annu Rev Physiol (2015) 77:131-60. doi:10.1146/ annurev-physiol-021014-071656

42. Morris MC. Nutrition and risk of dementia: overview and methodological issues. Ann N Y Acad Sci (2016) 1367:31-7. doi:10.1111/nyas.13047

43. Riffel APK, de Souza JA, Santos Mdo CQ, Horst A, Scheid T, Kolberg C, et al. Systemic administration of vitamins $\mathrm{C}$ and $\mathrm{E}$ attenuates nociception induced by chronic constriction injury of the sciatic nerve in rats. Brain Res Bull (2016) 121:169-77. doi:10.1016/j.brainresbull.2016.02.004

44. Barañano KW, Hartman AL. The ketogenic diet: uses in epilepsy and other neurologic illnesses. Curr Treat Options Neurol (2008) 10:410-9. doi:10.1007/ s1 1940-008-0043-8 
45. Lutas A, Yellen G. The ketogenic diet: metabolic influences on brain excitability and epilepsy. Trends Neurosci (2013) 36:32-40. doi:10.1016/j. tins.2012.11.005

46. Dupuis N, Curatolo N, Benoist J-F, Auvin S. Ketogenic diet exhibits anti-inflammatory properties. Epilepsia (2015) 56:e95-8. doi:10.1111/epi. 13038

47. Rahman M, Muhammad S, Khan MA, Chen H, Ridder DA, Müller-Fielitz H, et al. The $\beta$-hydroxybutyrate receptor HCA2 activates a neuroprotective subset of macrophages. Nat Commun (2014) 5:3944. doi:10.1038/ncomms4944

48. Offermanns S, Schwaninger M. Nutritional or pharmacological activation of HCA2 ameliorates neuroinflammation. Trends Mol Med (2015) 21:245-55. doi:10.1016/j.molmed.2015.02.002

49. Youm Y-H, Nguyen KY, Grant RW, Goldberg EL, Bodogai M, Kim D, et al. The ketone metabolite $\beta$-hydroxybutyrate blocks NLRP3 inflammasomemediated inflammatory disease. Nat Med (2015) 21:263-9. doi:10.1038/ nm.3804

50. Seyfried TN, Flores R, Poff AM, D’Agostino DP, Mukherjee P. Metabolic therapy: a new paradigm for managing malignant brain cancer. Cancer Lett (2015) 356:289-300. doi:10.1016/j.canlet.2014.07.015

51. Hertz L, Chen Y, Waagepetersen HS. Effects of ketone bodies in Alzheimer's disease in relation to neural hypometabolism, $\beta$-amyloid toxicity, and astrocyte function. J Neurochem (2015) 134:7-20. doi:10.1111/jnc.13107

52. Woolf EC, Scheck AC. The ketogenic diet for the treatment of malignant glioma. J Lipid Res (2015) 56:5-10. doi:10.1194/jlr.R046797

53. Wright C, Simone NL. Obesity and tumor growth: inflammation, immunity, and the role of a ketogenic diet. Curr Opin Clin Nutr Metab Care (2016) 19(4):294-9. doi:10.1097/MCO.0000000000000286

54. Kohli P, Levy BD. Resolvins and protectins: mediating solutions to inflammation.BrJ Pharmacol(2009)158:960-71.doi:10.1111/j.1476-5381.2009.00290.x

55. Li L, Wu Y, Wang Y, Wu J, Song L, Xian W, et al. Resolvin D1 promotes the interleukin-4-induced alternative activation in BV-2 microglial cells. J Neuroinflammation (2014) 11:72. doi:10.1186/1742-2094-11-72

56. Orr SK, Palumbo S, Bosetti F, Mount HT, Kang JX, Greenwood CE, et al. Unesterified docosahexaenoic acid is protective in neuroinflammation. J Neurochem (2013) 127:378-93. doi:10.1111/jnc.12392

57. Chang C-Y, Kuan Y-H, Li J-R, Chen W-Y, Ou Y-C, Pan H-C, et al. Docosahexaenoic acid reduces cellular inflammatory response following permanent focal cerebral ischemia in rats. J Nutr Biochem (2013) 24:2127-37. doi:10.1016/j.jnutbio.2013.08.004

58. Orr SK, Trépanier M-O, Bazinet RP. n-3 Polyunsaturated fatty acids in animal models with neuroinflammation. Prostaglandins Leukot Essent Fatty Acids (2013) 88:97-103. doi:10.1016/j.plefa.2012.05.008

59. Chang PK-Y, Khatchadourian A, McKinney RA, Maysinger D. Docosahexaenoic acid (DHA): a modulator of microglia activity and dendritic spine morphology. J Neuroinflammation (2015) 12:34. doi:10.1186/ s12974-015-0244-5

60. Tremblay M-E, Zhang I, Bisht K, Savage JC, Lecours C, Parent M, et al. Remodeling of lipid bodies by docosahexaenoic acid in activated microglial cells. J Neuroinflammation (2016) 13:116. doi:10.1186/s12974-016-0580-0

61. Welte MA. How brain fat conquers stress. Cell (2015) 163:269-70. doi:10.1016/j.cell.2015.09.046

62. Bailey AP, Koster G, Guillermier C, Hirst EMA, MacRae JI, Lechene CP, et al. Antioxidant role for lipid droplets in a stem cell niche of Drosophila. Cell (2015) 163:340-53. doi:10.1016/j.cell.2015.09.020

63. Ríos-Covián D, Ruas-Madiedo P, Margolles A, Gueimonde M, de los Reyes-Gavilán CG, Salazar N. Intestinal short chain fatty acids and their link with diet and human health. Front Microbiol (2016) 7:185. doi:10.3389/ fmicb.2016.00185

64. Tilg $\mathrm{H}$, Adolph TE. Influence of the human intestinal microbiome on obesity and metabolic dysfunction. Curr Opin Pediatr (2015) 27:496-501. doi:10.1097/MOP.0000000000000234

65. Chu H, Mazmanian SK. Winning the microbial battle, but not the war. Cell (2015) 163:271-2. doi:10.1016/j.cell.2015.09.050

66. Canfora EE, Jocken JW, Blaak EE. Short-chain fatty acids in control of body weight and insulin sensitivity. Nat Rev Endocrinol (2015) 11:577-91. doi:10.1038/nrendo.2015.128

67. Braniste V, Al-Asmakh M, Kowal C, Anuar F, Abbaspour A, Tóth M, et al. The gut microbiota influences blood-brain barrier permeability in mice. Sci Transl Med (2014) 6:263ra158. doi:10.1126/scitranslmed.3009759
68. Frost G, Sleeth ML, Sahuri-Arisoylu M, Lizarbe B, Cerdan S, Brody L, et al. The short-chain fatty acid acetate reduces appetite via a central homeostatic mechanism. Nat Commun (2014) 5:3611. doi:10.1038/ncomms4611

69. Sudo N, Chida Y, Aiba Y, Sonoda J, Oyama N, Yu X-N, et al. Postnatal microbial colonization programs the hypothalamic-pituitary-adrenal system for stress response in mice. J Physiol (2004) 558:263-75. doi:10.1113/ jphysiol.2004.063388

70. Bercik P, Collins SM. The effects of inflammation, infection and antibiotics on the microbiota-gut-brain axis. Adv Exp Med Biol (2014) 817:279-89. doi:10.1007/978-1-4939-0897-4_13

71. Dinan TG, Stanton C, Cryan JF. Psychobiotics: a novel class of psychotropic. Biol Psychiatry (2013) 74:720-6. doi:10.1016/j.biopsych.2013.05.001

72. Dobrovolskaia MA, McNeil SE. Immunological properties of engineered nanomaterials. Nat Nanotechnol (2007) 2:469-78. doi:10.1038/ nnano.2007.223

73. Colvin VL. The potential environmental impact of engineered nanomaterials. Nat Biotechnol (2003) 21:1166-70. doi:10.1038/nbt875

74. Frampton MW, Brauer M, Kleeman M, Kreyling WG, Ntziachristos L, Sarnat $\mathrm{SE}$, et al. Understanding the Health Effects of Ambient Ultrafine Particles. Boston, MA: Health Effects Institute (2013).

75. Zhang L, Webster TJ. Nanotechnology and nanomaterials: promises for improved tissue regeneration. Nano Today (2009) 4:66-80. doi:10.1016/j. nantod.2008.10.014

76. Liu H, Webster TJ. Nanomedicine for implants: a review of studies and necessary experimental tools. Biomaterials (2007) 28:354-69. doi:10.1016/j. biomaterials.2006.08.049

77. Ghanbari H, de Mel A, Seifalian AM. Cardiovascular application of polyhedral oligomeric silsesquioxane nanomaterials: a glimpse into prospective horizons. Int J Nanomedicine (2011) 6:775-86. doi:10.2147/IJN.S14881

78. Shevach M, Fleischer S, Shapira A, Dvir T. Gold nanoparticle-decellularized matrix hybrids for cardiac tissue engineering. Nano Lett (2014) 14:5792-6. doi:10.1021/nl502673m

79. Gustafson HH, Holt-Casper D, Grainger DW, Ghandehari H. Nanoparticle uptake: the phagocyte problem. Nano Today (2015) 10:487-510. doi:10.1016/j. nantod.2015.06.006

80. Kagan VE, Konduru NV, Feng W, Allen BL, Conroy J, Volkov Y, et al. Carbon nanotubes degraded by neutrophil myeloperoxidase induce less pulmonary inflammation. Nat Nanotechnol (2010) 5:354-9. doi:10.1038/nnano.2010.44

81. Park MVDZ, Neigh AM, Vermeulen JP, de la Fonteyne LJJ, Verharen HW, Briedé JJ, et al. The effect of particle size on the cytotoxicity, inflammation, developmental toxicity and genotoxicity of silver nanoparticles. Biomaterials (2011) 32:9810-7. doi:10.1016/j.biomaterials.2011.08.085

82. Park E-J, Yoon J, Choi K, Yi J, Park K. Induction of chronic inflammation in mice treated with titanium dioxide nanoparticles by intratracheal instillation. Toxicology (2009) 260:37-46. doi:10.1016/j.tox.2009.03.005

83. Ilinskaya AN, Dobrovolskaia MA. Understanding the immunogenicity and antigenicity of nanomaterials: past, present and future. Toxicol Appl Pharmacol (2016) 299:70-7. doi:10.1016/j.taap.2016.01.005

84. Longmire M, Choyke PL, Kobayashi H. Clearance properties of nano-sized particles and molecules as imaging agents: considerations and caveats. Nanomedicine (Lond) (2008) 3:703-17. doi:10.2217/17435889.3.5.703

85. Park HJ, Sohn J-H, Kim Y-J, Park YH, Han H, Park KH, et al. Acute exposure to silica nanoparticles aggravate airway inflammation: different effects according to surface characteristics. Exp Mol Med (2015) 47:e173. doi:10.1038/emm.2015.50

86. Dobrovolskaia MA. Pre-clinical immunotoxicity studies of nanotechnology-formulated drugs: challenges, considerations and strategy. J Control Release (2015) 220:571-83. doi:10.1016/j.jconrel.2015.08.056

87. Yanamala N, Kagan VE, Shvedova AA. Molecular modeling in structural nano-toxicology: interactions of nano-particles with nano-machinery of cells. Adv Drug Deliv Rev (2013) 65:2070-7. doi:10.1016/j.addr.2013.05.005

88. Papa S, Ferrari R, De Paola M, Rossi F, Mariani A, Caron I, et al. Polymeric nanoparticle system to target activated microglia/macrophages in spinal cord injury. J Control Release (2014) 174:15-26. doi:10.1016/j.jconrel. 2013.11.001

89. Cerqueira SR, Oliveira JM, Silva NA, Leite-Almeida H, Ribeiro-Samy S, Almeida A, et al. Microglia response and in vivo therapeutic potential of methylprednisolone-loaded dendrimer nanoparticles in spinal cord injury. Small (2013) 9:738-49. doi:10.1002/smll.201201888 
90. Hambardzumyan D, Gutmann DH, Kettenmann H. The role of microglia and macrophages in glioma maintenance and progression. Nat Neurosci (2016) 19:20-7. doi:10.1038/nn.4185

91. Louveau A, Smirnov I, Keyes TJ, Eccles JD, Rouhani SJ, Peske JD, et al. Structural and functional features of central nervous system lymphatic vessels. Nature (2015) 523:337-41. doi:10.1038/nature14432

92. Yi L, Xiao H, Xu M, Ye X, Hu J, Li F, et al. Glioma-initiating cells: a predominant role in microglia/macrophages tropism to glioma. J Neuroimmunol (2011) 232:75-82. doi:10.1016/j.jneuroim.2010.10.011

93. Lapa C, Linsenmann T, Lückerath K, Samnick S, Herrmann K, Stoffer C, et al. Tumor-associated macrophages in glioblastoma multiforme - a suitable target for somatostatin receptor-based imaging and therapy? PLoS One (2015) 10:e0122269. doi:10.1371/journal.pone.0122269

94. Szulzewsky F, Pelz A, Feng X, Synowitz M, Markovic D, Langmann T, et al. Glioma-associated microglia/macrophages display an expression profile different from M1 and M2 polarization and highly express Gpnmb and Spp1. PLoS One (2015) 10:e116644. doi:10.1371/journal.pone.0116644

95. Zhou B-BS, Zhang H, Damelin M, Geles KG, Grindley JC, Dirks PB. Tumourinitiating cells: challenges and opportunities for anticancer drug discovery. Nat Rev Drug Discov (2009) 8:806-23. doi:10.1038/nrd2137

96. Chen K, Huang Y, Chen J. Understanding and targeting cancer stem cells: therapeutic implications and challenges. Acta Pharmacol Sin (2013) 34:732-40. doi:10.1038/aps.2013.27

97. Bao S, Wu Q, McLendon RE, Hao Y, Shi Q, Hjelmeland AB, et al. Glioma stem cells promote radioresistance by preferential activation of the DNA damage response. Nature (2006) 444:756-60. doi:10.1038/nature05236

98. Hambardzumyan D, Squatrito M, Holland EC. Radiation resistance and stem-like cells in brain tumors. Cancer Cell (2007) 11:97. doi:10.1016/j. ccr.2006.12.011

99. Tokudome T, Sasaki A, Tsuji M, Udaka Y, Oyamada H, Tsuchiya H, et al. Reduced PTEN expression and overexpression of miR-17-5p, -19a-3p, $-19 b-3 p,-21-5 p,-130 b-3 p,-221-3 p$ and $-222-3 p$ by glioblastoma stem-like cells following irradiation. Oncol Lett (2015) 10:2269-72. doi:10.3892/ol. 2015.3594

100. Combs SE, Schmid TE, Vaupel P, Multhoff G. Stress response leading to resistance in glioblastoma - the need for innovative radiotherapy (iRT) concepts. Cancers (Basel) (2016) 8:15. doi:10.3390/cancers 8010015

101. Allen BG, Bhatia SK, Anderson CM, Eichenberger-Gilmore JM, Sibenaller ZA, Mapuskar KA, et al. Ketogenic diets as an adjuvant cancer therapy: history and potential mechanism. Redox Biol (2014) 2:963-70. doi:10.1016/j. redox.2014.08.002

102. Staedtke V, Bai R-Y, Laterra J. Investigational new drugs for brain cancer. Expert Opin Investig Drugs (2016) 1-20. doi:10.1080/13543784.2016. 1182497

103. Getts DR, Shea LD, Miller SD, King NJC. Harnessing nanoparticles for immune modulation. Trends Immunol (2015) 36:419-27. doi:10.1016/j. it.2015.05.007

104. Niu M, Naguib YW, Aldayel AM, Shi Y, Hursting SD, Hersh MA, et al. Biodistribution and in vivo activities of tumor-associated macrophage-targeting nanoparticles incorporated with doxorubicin. Mol Pharm (2014) 11:4425-36. doi:10.1021/mp500565q

105. Zhu S, Niu M, O’Mary H, Cui Z. Targeting of tumor-associated macrophages made possible by PEG-sheddable, mannose-modified nanoparticles. $\mathrm{Mol}$ Pharm (2013) 10:3525-30. doi:10.1021/mp400216r

106. Heek T, Kühne C, Depner H, Achazi K, Dernedde J, Haag R. Synthesis, photophysical, and biological evaluation of sulfated polyglycerol dendronized perylenebisimides (PBIs)-a promising platform for anti-inflammatory theranostic agents? Bioconjug Chem (2016) 27(3):727-36. doi:10.1021/acs. bioconjchem.5b00683

107. Reimann S, Gröger D, Kühne C, Riese SB, Dernedde J, Haag R. Shell cleavable dendritic polyglycerol sulfates show high anti-inflammatory properties by inhibiting L-selectin binding and complement activation. Adv Healthc Mater (2015) 4(14):2154-62. doi:10.1002/adhm.201500503

108. Maysinger D, Gröger D, Lake A, Licha K, Weinhart M, Chang PK-Y, et al. Dendritic polyglycerol sulfate inhibits microglial activation and reduces hippocampal CA1 dendritic spine morphology deficits. Biomacromolecules (2015) 16:3073-82. doi:10.1021/acs.biomac.5b00999

109. Getts DR, Terry RL, Getts MT, Deffrasnes C, Müller M, van Vreden C, et al. Therapeutic inflammatory monocyte modulation using immune-modifying microparticles. Sci Transl Med (2014) 6:219ra7. doi:10.1126/ scitranslmed.3007563

110. Smith MJ, Brown JM, Zamboni WC, Walker NJ. From immunotoxicity to nanotherapy: the effects of nanomaterials on the immune system. Toxicol Sci (2014) 138:249-55. doi:10.1093/toxsci/kfu005

111. Kettenmann H, Hanisch U-K, Noda M, Verkhratsky A. Physiology of microglia. Physiol Rev (2011) 91:461-553. doi:10.1152/physrev.00011.2010

112. Tambuyzer BR, Ponsaerts P, Nouwen EJ. Microglia: gatekeepers of central nervous system immunology. J Leukoc Biol (2009) 85:352-70. doi:10.1189/ jlb.0608385

113. Jones EV, Cook D, Murai KK. A neuron-astrocyte co-culture system to investigate astrocyte-secreted factors in mouse neuronal development. Methods Mol Biol (2012) 814:341-52. doi:10.1007/978-1-61779-452-0_22

114. Rodhe J. Cell culturing of human and murine microglia cell lines. In: Joseph B, Venero JL, editors. Microglia Methods in Molecular Biology. New York: Humana Press (2013). p. 11-6.

115. Gresa-Arribas N, Viéitez C, Dentesano G, Serratosa J, Saura J, Solà C. Modelling neuroinflammation in vitro: a tool to test the potential neuroprotective effect of anti-inflammatory agents. PLoS One (2012) 7:e45227. doi:10.1371/journal.pone.0045227

116. Saura J. Microglial cells in astroglial cultures: a cautionary note. J Neuroinflammation (2007) 4:26. doi:10.1186/1742-2094-4-26

117. Pluchino S, Zanotti L, Rossi B, Brambilla E, Ottoboni L, Salani G, et al. Neurosphere-derived multipotent precursors promote neuroprotection by an immunomodulatory mechanism. Nature (2005) 436:266-71. doi:10.1038/ nature03889

118. Pluchino S, Muzio L, Imitola J, Deleidi M, Alfaro-Cervello C, Salani G, et al. Persistent inflammation alters the function of the endogenous brain stem cell compartment. Brain (2008) 131:2564-78. doi:10.1093/brain/awn198

119. Li L, Walker TL, Zhang Y, Mackay EW, Bartlett PF. Endogenous interferon $\gamma$ directly regulates neural precursors in the non-inflammatory brain. J Neurosci (2010) 30:9038-50. doi:10.1523/JNEUROSCI.5691-09.2010

120. Cho S, Wood A, Bowlby MR. Brain slices as models for neurodegenerative disease and screening platforms to identify novel therapeutics. Curr Neuropharmacol (2007) 5:19-33. doi:10.2174/157015907780077105

121. Huuskonen J, Suuronen T, Miettinen R, van Groen T, Salminen A. A refined in vitro model to study inflammatory responses in organotypic membrane culture of postnatal rat hippocampal slices. J Neuroinflammation (2005) 2:25. doi:10.1186/1742-2094-2-25

122. Humpel C. Organotypic brain slice cultures: a review. Neuroscience (2015) 305:86-98. doi:10.1016/j.neuroscience.2015.07.086

123. De Simoni A, Yu L. Preparation of organotypic hippocampal slice cultures: interface method. Nat Protoc (2006) 1:1439-45. doi:10.1038/nprot.2006.228

124. Ting JT, Daigle TL, Chen Q, Feng G. Acute brain slice methods for adult and aging animals: application of targeted patch clampanalysis and optogenetics. Methods Mol Biol (2014) 1183:221-42. doi:10.1007/978-1-4939-1096-0_14

125. Mishra A, O'Farrell FM, Reynell C, Hamilton NB, Hall CN, Attwell D. Imaging pericytes and capillary diameter in brain slices and isolated retinae. Nat Protoc (2014) 9:323-36. doi:10.1038/nprot.2014.019

126. Buskila Y, Breen PP, Tapson J, van Schaik A, Barton M, Morley JW. Extending the viability of acute brain slices. Sci Rep (2014) 4:5309. doi:10.1038/srep05309

127. Oosterhof N, Boddeke E, van Ham TJ. Immune cell dynamics in the CNS: learning from the zebrafish. Glia (2015) 63:719-35. doi:10.1002/glia.22780

128. Buckwalter MS, Wyss-Coray T. Modelling neuroinflammatory phenotypes in vivo. J Neuroinflammation (2004) 1:10. doi:10.1186/1742-2094-1-10

129. Nazem A, Sankowski R, Bacher M, Al-Abed Y. Rodent models of neuroinflammation for Alzheimer's disease. J Neuroinflammation (2015) 12:74. doi:10.1186/s12974-015-0291-y

130. Cazareth J, Guyon A, Heurteaux C, Chabry J, Petit-Paitel A. Molecular and cellular neuroinflammatory status of mouse brain after systemic lipopolysaccharide challenge: importance of CCR2/CCL2 signaling. J Neuroinflammation (2014) 11:132. doi:10.1186/1742-2094-11-132

131. McCarthy RC, Lu D-Y, Alkhateeb A, Gardeck AM, Lee C-H, WesslingResnick M. Characterization of a novel adult murine immortalized microglial cell line and its activation by amyloid-beta. J Neuroinflammation (2016) 13:21. doi:10.1186/s12974-016-0484-Z

132. Butovsky O, Jedrychowski MP, Moore CS, Cialic R, Lanser AJ, Gabriely G, et al. Identification of a unique TGF- $\beta$-dependent molecular and functional signature in microglia. Nat Neurosci (2014) 17:131-43. doi:10.1038/nn.3599 
133. Greter M, Lelios I, Croxford AL. Microglia versus myeloid cell nomenclature during brain inflammation. Front Immunol (2015) 6:249. doi:10.3389/ fimmu.2015.00249

134. Morrison B III, Elkin BS, Dollé J-P, Yarmush ML. In vitro models of traumatic brain injury. Annu Rev Biomed Eng (2011) 13:91-126. doi:10.1146/ annurev-bioeng-071910-124706

135. Tang S-C, Arumugam TV, Xu X, Cheng A, Mughal MR, Jo DG, et al. Pivotal role for neuronal toll-like receptors in ischemic brain injury and functional deficits. Proc Natl Acad Sci U S A (2007) 104:13798-803. doi:10.1073/ pnas.0702553104

136. Latz E, Xiao TS, Stutz A. Activation and regulation of the inflammasomes. Nat Rev Immunol (2013) 13:397-411. doi:10.1038/nri3452

137. Gurung P, Lukens JR, Kanneganti T-D. Mitochondria: diversity in the regulation of the NLRP3 inflammasome. Trends Mol Med (2015) 21:193-201. doi:10.1016/j.molmed.2014.11.008

138. Hua K-F, Chou J-C, Ka S-M, Tasi Y-L, Chen A, Wu S-H, et al. Cyclooxygenase-2 regulates NLRP3 inflammasome-derived IL-1 $\beta$ production. J Cell Physiol (2015) 230:863-74. doi:10.1002/jcp.24815

139. Savage CD, Lopez-Castejon G, Denes A, Brough D. NLRP3-inflammasome activating DAMPs stimulate an inflammatory response in glia in the absence of priming which contributes to brain inflammation after injury. Inflammation (2012) 3:288. doi:10.3389/fimmu.2012.00288

140. Goldmann T, Tay TL, Prinz M. Love and death: microglia, NLRP3 and the Alzheimer's brain. Cell Res (2013) 23:595-6. doi:10.1038/cr.2013.24

141. Gustin A, Kirchmeyer M, Koncina E, Felten P, Losciuto S, Heurtaux T, et al. NLRP3 inflammasome is expressed and functional in mouse brain microglia but not in astrocytes. PLoS One (2015) 10:e0130624. doi:10.1371/journal. pone. 0130624

142. Liu H-D, Li W, Chen Z-R, Hu Y-C, Zhang D-D, Shen W, et al. Expression of the NLRP3 inflammasome in cerebral cortex after traumatic brain injury in a rat model. Neurochem Res (2013) 38:2072-83. doi:10.1007/s11064-013-1115-Z

143. Jha S, Srivastava SY, Brickey WJ, Iocca H, Toews A, Morrison JP, et al. The inflammasome sensor, NLRP3, regulates CNS inflammation and demyelination via caspase-1 and interleukin-18. J Neurosci (2010) 30:15811-20. doi:10.1523/JNEUROSCI.4088-10.2010

144. Tovar-Y-Romo LB, Penagos-Puig A, Ramírez-Jarquín JO. Endogenous recovery after brain damage: molecular mechanisms that balance neuronal life/death fate. J Neurochem (2016) 136:13-27. doi:10.1111/jnc.13362

145. Tan M-S, Tan L, Jiang T, Zhu X-C, Wang H-F, Jia C-D, et al. Amyloid- $\beta$ induces NLRP1-dependent neuronal pyroptosis in models of Alzheimer's disease. Cell Death Dis (2014) 5:e1382. doi:10.1038/cddis.2014.348

146. Sagulenko V, Thygesen SJ, Sester DP, Idris A, Cridland JA, Vajjhala PR, et al. AIM2 and NLRP3 inflammasomes activate both apoptotic and pyroptotic death pathways via ASC. Cell Death Differ (2013) 20:1149-60. doi:10.1038/ cdd.2013.37

147. Scheller J, Chalaris A, Schmidt-Arras D, Rose-John S. The pro- and anti-inflammatory properties of the cytokine interleukin-6. Biochim Biophys Acta (2011) 1813:878-88. doi:10.1016/j.bbamcr.2011.01.034

148. Smith JA, Das A, Ray SK, Banik NL. Role of pro-inflammatory cytokines released from microglia in neurodegenerative diseases. Brain Res Bull (2012) 87:10-20. doi:10.1016/j.brainresbull.2011.10.004

149. Scheller J, Garbers C, Rose-John S. Interleukin-6: from basic biology to selective blockade of pro-inflammatory activities. Semin Immunol (2014) 26:2-12. doi:10.1016/j.smim.2013.11.002

150. Schaper F, Rose-John S. Interleukin-6: biology, signaling and strategies of blockade. Cytokine Growth Factor Rev (2015) 26:475-87. doi:10.1016/j. cytogfr.2015.07.004

151. Hong S-S, Choi JH, Lee SY, Park Y-H, Park K-Y, Lee JY, et al. A novel small-molecule inhibitor targeting the IL- 6 receptor $\beta$ subunit, glycoprotein 130. J Immunol (2015) 195:237-45. doi:10.4049/jimmunol.1402908

152. Rivest S. Molecular insights on the cerebral innate immune system. Brain Behav Immun (2003) 17:13-9. doi:10.1016/S0889-1591(02)00055-7

153. Espinosa-Oliva AM, de Pablos RM, Herrera AJ. Intracranial injection of LPS in rat as animal model of neuroinflammation. Methods Mol Biol (2013) 1041:295-305. doi:10.1007/978-1-62703-520-0_26

154. Opal SM, Laterre P-F, Francois B, LaRosa SP, Angus DC, Mira J-P, et al. Effect of eritoran, an antagonist of MD2-TLR4, on mortality in patients with severe sepsis: the ACCESS randomized trial. JAMA (2013) 309:1154-62. doi:10.1001/jama.2013.2194
155. Smith C, Wang X, Yin H. Caspases come together over LPS. Trends Immunol (2015) 36:59-61. doi:10.1016/j.it.2014.12.007

156. Galluzzi L, López-Soto A, Kumar S, Kroemer G. Caspases connect celldeath signaling to organismal homeostasis. Immunity (2016) 44:221-31. doi:10.1016/j.immuni.2016.01.020

157. Shi J, Zhao Y, Wang Y, Gao W, Ding J, Li P, et al. Inflammatory caspases are innate immune receptors for intracellular LPS. Nature (2014) 514:187-92. doi:10.1038/nature13683

158. Yokoo H, Chiba S, Tomita K, Takashina M, Sagara H, Yagisita S, et al. Neurodegenerative evidence in mice brains with cecal ligation and puncture-induced sepsis: preventive effect of the free radical scavenger edaravone. PLoS One (2012) 7:e51539. doi:10.1371/journal.pone.0051539

159. Dejager L, Pinheiro I, Dejonckheere E, Libert C. Cecal ligation and puncture: the gold standard model for polymicrobial sepsis? Trends Microbiol (2011) 19:198-208. doi:10.1016/j.tim.2011.01.001

160. Rittirsch D, Huber-Lang MS, Flierl MA, Ward PA. Immunodesign of experimental sepsis by cecal ligation and puncture. Nat Protoc (2009) 4:31-6. doi:10.1038/nprot.2008.214

161. Sawyer RG. Surgical Infections, An Issue of Surgical Clinics. Philadelphia, PA: Elsevier Health Sciences (2015).

162. Jankowsky JL, Slunt HH, Ratovitski T, Jenkins NA, Copeland NG, Borchelt DR. Co-expression of multiple transgenes in mouse CNS: a comparison of strategies. Biomol Eng (2001) 17:157-65. doi:10.1016/ S1389-0344(01)00067-3

163. Kumar DKV, Choi SH, Washicosky KJ, Eimer WA, Tucker S, Ghofrani J, et al. Amyloid- $\beta$ peptide protects against microbial infection in mouse and worm models of Alzheimer's disease. Sci Transl Med (2016) 8:340ra72. doi:10.1126/ scitranslmed.aaf1059

164. St Martin JL, Klucken J, Outeiro TF, Nguyen P, Keller-McGandy C, CantutiCastelvetri I, et al. Dopaminergic neuron loss and up-regulation of chaperone protein mRNA induced by targeted over-expression of alpha-synuclein in mouse substantia nigra. J Neurochem (2007) 100:1449-57. doi:10.1111/j.14714159.2006.04310.x

165. Gurney ME, Pu H, Chiu AY, Dal Canto MC, Polchow CY, Alexander DD, et al. Motor neuron degeneration in mice that express a human $\mathrm{Cu}, \mathrm{Zn}$ superoxide dismutase mutation. Science (1994) 264:1772-5. doi:10.1126/ science. 8209258

166. Wong PC, Pardo CA, Borchelt DR, Lee MK, Copeland NG, Jenkins NA, et al. An adverse property of a familial ALS-linked SOD1 mutation causes motor neuron disease characterized by vacuolar degeneration of mitochondria. Neuron (1995) 14:1105-16. doi:10.1016/0896-6273(95)90259-7

167. Kozai TDY, Li X, Bodily LM, Caparosa EM, Zenonos GA, Carlisle DL, et al. Effects of caspase-1 knockout on chronic neural recording quality and longevity: insight into cellular and molecular mechanisms of the reactive tissue response. Biomaterials (2014) 35:9620-34. doi:10.1016/j.biomaterials.2014. 08.006

168. Swiech L, Heidenreich M, Banerjee A, Habib N, Li Y, Trombetta J, et al. In vivo interrogation of gene function in the mammalian brain using CRISPR-Cas9. Nat Biotechnol (2015) 33:102-6. doi:10.1038/ nbt.3055

169. Yen S-T, Zhang M, Deng JM, Usman SJ, Smith CN, Parker-Thornburg J, et al. Somatic mosaicism and allele complexity induced by CRISPR/Cas 9 RNA injections in mouse zygotes. Dev Biol (2014) 393:3-9. doi:10.1016/j. ydbio.2014.06.017

170. Maysinger D, Ji J, Hutter E, Cooper E. Nanoparticle-based and bioengineered probes and sensors to detect physiological and pathological biomarkers in neural cells. Front Neurosci (2015) 9:480. doi:10.3389/fnins. 2015.00480

171. Mossakowski AA, Pohlan J, Bremer D, Lindquist R, Millward JM, Bock M, et al. Tracking CNS and systemic sources of oxidative stress during the course of chronic neuroinflammation. Acta Neuropathol (2015) 130:799-814. doi:10.1007/s00401-015-1497-x

172. Radbruch H, Bremer D, Mothes R, Günther R, Rinnenthal JL, Pohlan J, et al. Intravital FRET: probing cellular and tissue function in vivo. Int J Mol Sci (2015) 16:11713-27. doi:10.3390/ijms160511713

173. Zhang F, Mastorakos P, Mishra MK, Mangraviti A, Hwang L, Zhou J, et al. Uniform brain tumor distribution and tumor associated macrophage targeting of systemically administered dendrimers. Biomaterials (2015) 52:507-16. doi:10.1016/j.biomaterials.2015.02.053 
174. Spitzer N, Sammons GS, Price EM. Autofluorescent cells in rat brain can be convincing impostors in green fluorescent reporter studies. J Neurosci Methods (2011) 197:48-55. doi:10.1016/j.jneumeth.2011.01.029

175. Neumann M, Gabel D. Simple method for reduction of autofluorescence in fluorescence microscopy. J Histochem Cytochem (2002) 50:437-9. doi: $10.1177 / 002215540205000315$

176. Goldey GJ, Roumis DK, Glickfeld LL, Kerlin AM, Reid RC, Bonin V, et al. Removable cranial windows for long-term imaging in awake mice. Nat Protoc (2014) 9:2515-38. doi:10.1038/nprot.2014.165

177. Holtmaat A, Bonhoeffer T, Chow DK, Chuckowree J, De Paola V, Hofer SB, et al. Long-term, high-resolution imaging in the mouse neocortex through a chronic cranial window. Nat Protoc (2009) 4:1128-44. doi:10.1038/ nprot.2009.89

178. Dorand RD, Barkauskas DS, Evans TA, Petrosiute A, Huang AY. Comparison of intravital thinned skull and cranial window approaches to study CNS immunobiology in the mouse cortex. Intravital (2014) 3:e29728. doi:10.4161/ intv.29728

179. Yang G, Pan F, Parkhurst CN, Grutzendler J, Gan W-B. Thinned-skull cranial window technique for long-term imaging of the cortex in live mice. Nat Protoc (2010) 5:201-8. doi:10.1038/nprot.2009.222

180. Yu X, Zuo Y. Two-photon in vivo imaging of dendritic spines in the mouse cortex using a thinned-skull preparation. J Vis Exp (2014) (87):e51520. doi: $10.3791 / 51520$

181. Aswendt M, Adamczak J, Couillard-Despres S, Hoehn M. Boosting bioluminescence neuroimaging: an optimized protocol for brain studies. PLoS One (2013) 8:e55662. doi:10.1371/journal.pone.0055662

182. Cordeau P, Lalancette-Hébert M, Weng YC, Kriz J. Estrogen receptors alpha mediates postischemic inflammation in chronically estrogen-deprived mice. Neurobiol Aging (2016) 40:50-60. doi:10.1016/j.neurobiolaging. 2016.01.002

183. Dany Arsenault JD-O. A novel combinational approach of microstimulation and bioluminescence imaging to study the mechanisms of action of cerebral electrical stimulation in mice: stimulation device for small animals. J Physiol (2015) 593(Pt 10):2257-78. doi:10.1113/jphysiol.2014.287243

184. Lalancette-Hébert M, Moquin A, Choi AO, Kriz J, Maysinger D. Lipopolysaccharide-QD micelles induce marked induction of TLR2 and lipid droplet accumulation in olfactory bulb microglia. Mol Pharm (2010) 7:1183-94. doi:10.1021/mp1000372

185. Moquin A, Hutter E, Choi AO, Khatchadourian A, Castonguay A, Winnik FM, et al. Caspase-1 activity in microglia stimulated by pro-inflammagen nanocrystals. ACS Nano (2013) 7:9585-98. doi:10.1021/nn404473g

186. Maysinger D, Behrendt M, Lalancette-Hébert M, Kriz J. Real-time imaging of astrocyte response to quantum dots: in vivo screening model system for biocompatibility of nanoparticles. Nano Lett (2007) 7:2513-20. doi:10.1021/ nl071611t

187. Maysinger D, Hutter E. Nanoparticle-based caspase sensors. Nanomedicine (Lond) (2015) 10:483-501. doi:10.2217/nnm.14.158
188. Ding Y, Li J, Enterina JR, Shen Y, Zhang I, Tewson PH, et al. Ratiometric biosensors based on dimerization-dependent fluorescent protein exchange. Nat Methods (2015) 12:195-8. doi:10.1038/nmeth.3261

189. Kong L, Tang J, Little JP, Yu Y, Lämmermann T, Lin CP, et al. Continuous volumetric imaging via an optical phase-locked ultrasound lens. Nat Methods (2015) 12:759-62. doi:10.1038/nmeth.3476

190. Park J-H, Sun W, Cui M. High-resolution in vivo imaging of mouse brain through the intact skull. Proc Natl Acad Sci U S A (2015) 112:9236-41. doi:10.1073/pnas.1505939112

191. Govorunova EG, Sineshchekov OA, Janz R, Liu X, Spudich JL. Natural light-gated anion channels: a family of microbial rhodopsins for advanced optogenetics. Science (2015) 349:647-50. doi:10.1126/science.aaa7484

192. Deisseroth K. Optogenetics: 10 years of microbial opsins in neuroscience. Nat Neurosci (2015) 18:1213-25. doi:10.1038/nn.4091

193. Berndt A, Lee SY, Wietek J, Ramakrishnan C, Steinberg EE, Rashid AJ, et al. Structural foundations of optogenetics: determinants of channelrhodopsin ion selectivity. Proc Natl Acad Sci U S A (2016) 113:822-9. doi:10.1073/ pnas. 1523341113

194. Figueiredo M, Lane S, Tang F, Liu BH, Hewinson J, Marina N, et al. Optogenetic experimentation on astrocytes. Exp Physiol (2011) 96:40-50. doi:10.1113/expphysiol.2010.052597

195. Vazey EM, Aston-Jones G. New tricks for old dogmas: optogenetic and designer receptor insights for Parkinson's disease. Brain Res (2013) 1511:153-63. doi:10.1016/j.brainres.2013.01.021

196. Mao G, Qu F, St. Croix CM, Tyurina YY, Planas-Iglesias J, Jiang J, et al. Mitochondrial redox opto-lipidomics reveals mono-oxygenated cardiolipins as pro-apoptotic death signals. ACS Chem Biol (2016) 11:530-40. doi:10.1021/ acschembio. 5 b00737

197. Gregory AE, Titball R, Williamson D. Vaccine delivery using nanoparticles. Front Cell Infect Microbiol (2013) 3:13. doi:10.3389/fcimb.2013.00013

198. Dunkle A, Blanchette C, Boone T, Corzett M, Fischer N, Hoeprich P, et al. Co-delivery of adjuvant and subunit antigens via a nanoparticle platform induces tissue-associated and systemic adaptive immune responses (P4409). J Immunol (2013) 190:205.14.

199. Zhao L, Seth A, Wibowo N, Zhao C-X, Mitter N, Yu C, et al. Nanoparticle vaccines. Vaccine (2014) 32:327-37. doi:10.1016/j.vaccine.2013.11.069

Conflict of Interest Statement: The authors declare that the research was conducted in the absence of any commercial or financial relationships that could be construed as a potential conflict of interest.

Copyright (c) 2016 Maysinger and Zhang. This is an open-access article distributed under the terms of the Creative Commons Attribution License (CC BY). The use, distribution or reproduction in other forums is permitted, provided the original author(s) or licensor are credited and that the original publication in this journal is cited, in accordance with accepted academic practice. No use, distribution or reproduction is permitted which does not comply with these terms. 


\title{
Imaging of Leukocyte Trafficking in Alzheimer's Disease
}

\author{
Enrica Pietronigro, Elena Zenaro and Gabriela Constantin* \\ Section of General Pathology, Department of Medicine, University of Verona, Verona, Italy
}

Alzheimer's disease (AD) is the most common neurodegenerative disorder and is characterized by a progressive decline of cognitive functions. The neuropathological features of $A D$ include amyloid beta $(A \beta)$ deposition, intracellular neurofibrillary tangles derived from the cytoskeletal hyperphosphorylated tau protein, amyloid angiopathy, the loss of synapses, and neuronal degeneration. In the last decade, inflammation has emerged as a key feature of $A D$, but most studies have focused on the role of microglia-driven neuroinflammation mechanisms. A dysfunctional blood-brain barrier has also been implicated in the pathogenesis of $A D$, and several studies have demonstrated that the vascular deposition of $A \beta$ induces the expression of adhesion molecules and alters the expression of tight junction proteins, potentially facilitating the transmigration of circulating leukocytes. Two-photon laser scanning microscopy (TPLSM) has become an indispensable tool to dissect the molecular mechanisms controlling leukocyte trafficking in the central nervous system (CNS). Recent TPLSM studies have shown that vascular deposition of $A \beta$ in the CNS promotes intraluminal neutrophil adhesion and crawling on the brain endothelium and also that neutrophils extravasate in the parenchyma preferentially in areas with $A \beta$ deposits. These studies have also highlighted a role for LFA-1 integrin in neutrophil accumulation in the CNS of AD-like disease models, revealing that LFA-1 inhibition reduces the corresponding cognitive deficit and AD neuropathology. In this article, we consider how current imaging techniques can help to unravel new inflammation mechanisms in the pathogenesis of $A D$ and identify novel therapeutic strategies to treat the disease by interfering with leukocyte trafficking mechanisms.

Keywords: Alzheimer's disease, leukocyte trafficking, two-photon laser scanning microscopy

Specialty section:

This article was submitted to Multiple Sclerosis and Neuroimmunology,

a section of the journal

Frontiers in Immunology

Received: 30 November 2015

Accepted: 23 January 2016

Published: 15 February 2016

Citation:

Pietronigro E, Zenaro E and Constantin G (2016) Imaging of

Leukocyte Trafficking in

Alzheimer's Disease.

Front. Immunol. 7:33.

doi: 10.3389/fimmu.2016.00033

\section{INTRODUCTION}

Alzheimer's disease (AD) is the most common neurodegenerative cause of dementia in the elderly and is characterized by a progressive deterioration of cognitive functions. The neuropathological features include amyloid beta (A $\beta$ ) neuritic plaques, neurofibrillary tangles (NFTs) comprising aggregates of hyperphosphorylated microtubule tau protein, amyloid angiopathy, and the loss of neurons and synapses (1). The major pathogenic concept in the field of AD research is the amyloid cascade hypothesis, which states that the sequence of pathological events leading to $\mathrm{AD}$ is characterized by the accumulation of $A \beta$ peptides resulting from the aberrant processing of amyloid precursor protein (APP) and dysfunctional A $\beta$ clearance, followed by the deposition of NFTs and the onset of synaptic dysfunction and neuronal loss. A $\beta$ peptides are formed by the proteolytic cleavage of APP due to the sequential activities of $\beta$-site APP-cleaving enzyme 1 (BACE-1) ( $\beta$-secretase), $\gamma$-secretase, 
and a protein complex with presenilin 1 (PS1) at its catalytic core. Although amyloid plaques and aggregated tau are both part of the neuropathological definition of the disease, numerous studies suggest that soluble oligomeric forms of $A \beta$ and tau are the predominant mediators of cytotoxicity in $\mathrm{AD}(2)$.

Alzheimer's disease pathology is also characterized by an inflammatory response primarily driven by cytokines and the intrinsic myeloid cells in the brain, which are known as microglia (3). It is now widely accepted that microglia-mediated neuroinflammatory responses may promote the neurodegeneration observed in $\mathrm{AD}(1,3)$. Microglial activation precedes neuropil loss in $\mathrm{AD}$ patients, and recent genome-wide association studies have revealed that microglial genes, such as CD33, TREM2, and $H L A-D R$, are associated with susceptibility to the late-onset form of the disease (3). Furthermore, in response to A $\beta$ or NFTs, microglial cells produce proinflammatory cytokines, chemokines, and complement peptides, which can recruit leukocyte subpopulations to the brain. $A \beta$ also stimulates microglia to produce reactive nitrogen intermediates, such as nitric oxide (NO) and reactive oxygen species (ROS), and the resulting oxidative stress induces neuronal damage (3).

Circulating leukocytes may also play a role in the inflammation process during $\mathrm{AD}$. The migration of leukocytes from blood vessels into the central nervous system (CNS) involves a sequence of adhesion and activation events including (1) capture (tethering) and rolling, which are mediated by the interactions between selectins and mucins, and/or between integrins and proteins with immunoglobulin domains; (2) activation induced by chemokines, resulting in the subsequent activation of integrins; (3) arrest mediated by integrins and their counter-ligands; and (4) diapedesis or transmigration (4). Additional steps may include slow rolling, adhesion strengthening and spreading, and intravascular crawling (5). Leukocyte trafficking in the CNS during inflammatory diseases is mediated predominantly by endothelial E-selectin, P-selectin, and their mucin ligands, as well as leukocyte integrins including $\alpha 4 \beta 1$ (also known as very late antigen 4 , VLA-4) and $\alpha \mathrm{L} \beta 2$ (also known as leukocyte functionassociated antigen 1, LFA-1), which bind the endothelial vascular cell adhesion molecule (VCAM-1) and intercellular cell adhesion molecule (ICAM-1), respectively.

The role of circulating immune system cells in AD-related brain damage is poorly understood, but the use of in vivo imaging techniques, such as two-photon laser scanning microscopy (TPLSM), can provide insights into the mechanisms controlling leukocyte trafficking in $\mathrm{AD}$ and may lead to the development of novel therapeutic strategies to delay the progression of the disease. In this review, we discuss recent work on the role of circulating leukocytes in $\mathrm{AD}$, highlighting the use of in vivo imaging to investigate leukocyte recruitment in the CNS and to study the basis of novel disease mechanisms.

\section{LEUKOCYTE TRAFFICKING IN AD}

Monocytes are circulating leukocytes that play an important role in the innate immune response against pathogens. Numerous studies have shown that peripheral myeloid cells can infiltrate brain tissue and reduce the deposition of $A \beta$ plaques (6-9).
The entry of monocytes into the CNS is tightly regulated and involves the CC-chemokine ligand 2 (CCL2)-CCR2 axis (10). $\mathrm{A} \beta$ is chemotactic for monocytes, and it induces the secretion of proinflammatory cytokines and monocyte transendothelial migration in a blood-brain barrier (BBB) model, in a process that involves the $\mathrm{A} \beta$ receptor (RAGE) and platelet endothelial cell adhesion molecule (PECAM-1) expressed on endothelial cells $(11,12)$. In agreement with these in vitro studies, the injection of synthetic A $\beta$ peptides into the hippocampus triggers the trafficking of bone-marrow-derived monocytic cells into the brain, which then differentiate into ramified microglia and penetrate into the core of $A \beta$ plaques $(7,13)$. Furthermore, recent data indicate that infiltrating monocytes rather than resident microglia express TREM2, a receptor involved in myeloid cell phagocytosis, further supporting the role of peripheral myeloid cells in AD pathogenesis (14). Intravital TPLSM has elegantly confirmed that patrolling monocytes are attracted to and crawl onto the luminal walls of $A \beta$-positive veins but not $A \beta$-positive arteries or A $\beta$-negative blood vessels (15).

Neutrophils are the most abundant population of cells in the blood and are the primary mediators of the innate immune response. Previous reports $(16,17)$, including our own results (18), have shown that neutrophils do not necessarily need to accumulate in tissues in high numbers in order to induce tissue damage: intravascular adhesion per se without transmigration is sufficient to induce endothelial injury. The role of neutrophils in the induction of neuropathological changes and memory deficit in $\mathrm{AD}$ models has been demonstrated only recently (19). A higher number of infiltrating neutrophils was observed at the onset of cognitive deficits in 5xFAD and 3xTg-AD mice, especially in the cortex and hippocampus. In corresponding TPLSM studies, neutrophil extravasation was observed at the early stage of the disease inside the cerebral parenchyma, particularly in areas adjacent to vascular $A \beta$ deposits or rich in intraparenchymal $A \beta$ plaques (19). Similarly, Gr1+-labeled cells also infiltrate the brain parenchyma of $5 x F A D$ mice and migrate toward $\mathrm{A} \beta$ plaques $(20)$. These data, together with previous in vitro results, suggest that $A \beta$ plays a role in the chemotaxis and accumulation of neutrophils in the brains of $\mathrm{AD}$ mice $(21,22)$. Furthermore, soluble $\mathrm{A} \beta$ oligomers rapidly trigger neutrophil adhesion to integrin ligands in vitro and induce the transition of LFA-1 integrin from the low- to the high-affinity binding state, suggesting that $\mathrm{A} \beta$ plays a key role in neutrophil intravascular adhesion and migration into the brain during AD (19). Neutrophils migrate into the brains of mice with AD-like disease by using LFA-1 integrin, which controls both intravascular adhesion and intraparenchymal motility. Indeed, the treatment of $\mathrm{AD}$-like transgenic mice with monoclonal antibodies that block LFA-1 integrin or deplete neutrophils at early stages of the disease (when mice start to present memory impairment) mitigated the neuropathological hallmarks of $\mathrm{AD}$ and reversed the cognitive deficits. Most importantly from a therapeutic perspective, blocking neutrophil adhesion during the early stages of the disease provided a long-term beneficial effect on cognition in older mice (19).

The presence of neutrophils in the brains of $\mathrm{AD}$ subjects was previously suggested by the presence of cells expressing cathepsin G, a protease produced specifically by neutrophils, within the 
$\mathrm{AD}$ brain parenchyma and inside cerebral blood vessels (23). Additionally, the presence of CAP37, an inflammatory mediator expressed in neutrophils, was reported in the blood vessels and hippocampal vasculature of $\mathrm{AD}$ patients $(24,25)$. However, the first definitive evidence for the presence of neutrophils in the $\mathrm{AD}$ brain was our confirmation that myeloperoxidase (MPO) ${ }^{+}$ cells are localized in areas with $A \beta$ deposits (19). These cells were typically found around the periphery of $A \beta$ plaques at a distance of $<50 \mu \mathrm{m}$, and their non-random distribution suggested that $A \beta$ influences the microenvironmental positioning of neutrophils inside the $\mathrm{AD}$ brain.

Several reports indicate that $\mathrm{T}$ cells also accumulate in the AD brain (26-29). For example, a greater number of activated $\mathrm{CD}^{+}$and $\mathrm{CD} 8^{+} \mathrm{T}$ cells was observed in the blood, adhering to the vascular endothelium or migrating into the parenchyma of $\mathrm{AD}$ patients, compared to healthy controls or patients with other types of dementia $(4,26-29)$. Notably, the majority of $\mathrm{T}$ cells in $\mathrm{AD}$ brain tissue are located in the hippocampus and other limbic structures, which are among those regions most affected in $\mathrm{AD}$ (28). In support of these data, enhanced activated $\mathrm{CD} 4^{+}$and $\mathrm{CD} 8^{+}$ were recently identified in the cerebrospinal fluid (CSF) of individuals with mild cognitive impairment (MCI) and patients with mild $\mathrm{AD}$, with the proportion of activated $\mathrm{CD} 8^{+} \mathrm{T}$ cells showing the greatest increase (30). In agreement with human data, mouse studies have shown that $\mathrm{T}$ cells infiltrate the brains of APP/PS1 mice, and a proportion of these cells secrete interferon (IFN)- $\gamma$ or interleukin (IL)-17 (31). This suggests that the inflammatory response stimulated by $\mathrm{T}$ cells that have migrated into the $\mathrm{AD}$ brain may activate microglia and astrocytes and may recruit other inflammatory cells that are potentially harmful to the CNS, thus exacerbating the pathogenesis of $\mathrm{AD}$.

Taken together, the studies discussed in this section suggest that a role for circulating leukocytes in $\mathrm{AD}$ is becoming clearer, but further studies are needed to determine the impact of specific immune cell populations on the cognitive deficit and neuropathological changes in AD. In this context, future TPLSM studies may provide key data to increase our understanding of the mechanisms controlling leukocyte trafficking in the $\mathrm{AD}$ brain, as well as interactions between migrating leukocytes and CNSresident cells.

\section{TPLSM - THE GENERAL CONTEXT}

The optical principles of two-photon microscopy are based on the absorption of two longer-wavelength lower-energy photons as a single quantum of energy by a fluorophore, thus promoting an electron to an excited state (32). TPLSM offers several advantages over traditional forms of microscopy for the investigation of living systems because it provides three-dimensional deep-tissue images and single-cell spatiotemporal information that other imaging techniques cannot achieve (33-35). TPLSM is particularly suitable for high-resolution imaging in intact thick tissues, such as whole organs, brain slices, embryos, and live animals (intravital imaging). Extensive tissue penetration is possible due to the reduced scattering of the infrared (IR) excitation light compared to one-photon confocal microscopy. The restriction of two-photon excitation solely to the focal plane provides most of the advantages over traditional confocal microscopy. TPLSM generates fluorescence only within the focal plane, thus substantially reducing photobleaching and photodamage outside the excitation volume (which represents only a small proportion of the overall sample), and thereby prolonging the viability of specimens especially during long-term imaging.

The spatiotemporal dynamics of leukocyte trafficking can be investigated in vivo using cutting-edge TPLSM technology (36, 37). This technique has changed our static view of the immune system and allowed the dissection of leukocyte migration behavior and cell-cell contacts, which are fundamental requirements for an effective immune response $(38,39)$. Several aspects of leukocyte migration that could not be predicted using in vitro systems have been identified, thanks to this advanced technology, including leukocyte intravascular crawling and the directional movement and polarization of emigrated leukocytes along an extravascular chemokine gradient $(40,41)$.

\section{TPLSM IN THE AD BRAIN}

Two-photon laser scanning microscopy has recently contributed to several developments in the field of neuroscience, facilitating studies of cell morphology and function in the living brain. TPLSM has become increasingly necessary to study structural and functional changes in the living brain because the imaging of neurons, glia, and vasculature provides new insights into the function of the CNS under physiological and pathophysiological conditions (42-45). Initial studies investigated the structural plasticity of dendritic spines and the axons of pyramidal neurons in the mouse cortex and how their changes could influence long-term information storage $(46,47)$. TPLSM also revealed the dynamic structure of microglial cells that constantly survey the brain parenchyma and switch their behavior to an activated state immediately after injury (48). In addition to such morphological studies, TPLSM has also been used for calcium imaging, thus improving the analysis of neuronal signaling and plasticity $(49,50)$.

In the context of $\mathrm{AD}$, intravital imaging in the brain has mainly been used to study amyloid plaque deposition, dendritic spine loss, and microglial aggregation around A $\beta$ plaques $(6,51-55)$. TPLSM has allowed the repeated visualization of the same amyloid plaques labeled with fluorescent dyes to evaluate their growth in transgenic mouse models of $\operatorname{AD}(51,55,56)$. A refined TPLSM method was used to define the kinetics of amyloid plaque growth in Tg2576 mice (57). The simultaneous imaging of amyloid plaques and neurons labeled with viral-expressed green fluorescent protein (GFP) in a transgenic mouse model of $\mathrm{AD}$ highlighted the detrimental effect of $A \beta$ on the neuronal circuitry (58).

Recent TPLSM studies have confirmed that dendritic spine loss after newborn amyloid deposits persists in the proximity of amyloid plaques in APP/PS1 mice (59). TPLSM imaging has also revealed that the depletion of $A \beta$ in the brains of PDAPP-YFP transgenic mice treated with anti-A $\beta$ antibodies promotes the rapid recovery of existing amyloid-associated neuritic dystrophy in vivo, indicating that axonal and dendritic damage is a partially reversible phenomenon (60).

The availability of transgenic mice expressing fluorescent cells, such as microglia, has provided a more intimate view of the 
interactions between amyloid plaques, neuronal structures, and cells in the brain parenchyma during the progression of $\mathrm{AD}$ (61) or astrocytes (62). The rapid appearance of new amyloid plaques induces progressive changes in neurites, manifesting as dendritic and axonal abnormalities, and the activation and recruitment of microglia to areas with amyloid plaques (63).

Two-photon laser scanning microscopy has also facilitated the characterization of impaired microglial functionality near amyloid deposits in $\mathrm{AD}$ mice, suggesting a correlation between plaque deposition and microglial behavior (64).

Two-photon laser scanning microscopy analysis of leukocyte trafficking in the inflamed CNS has focused almost exclusively on infections, stroke, and experimental autoimmune encephalomyelitis (EAE) (65-69). However, recent TPLSM studies have shown that circulating leukocytes migrate into the brain parenchyma of AD mice, revealing previously unknown mechanisms of $\mathrm{AD}$ pathogenesis and helping to identify new therapeutic strategies for $\operatorname{AD}(19,20)$. Our recent TPLSM experiments have demonstrated that circulating neutrophils arrest and perform intraluminal crawling preferentially inside blood vessels with $A \beta$ deposits, supporting a role for $A \beta$ in leukocyte migration into the brain (19). Furthermore, we have recently shown using TPLSM that neutrophils isolated from LFA-1-deficient mice cannot adhere to the brain vessels and therefore extravasate in the brain parenchyma, suggesting that LFA-1 integrin is a key mediator of neutrophil trafficking in $\mathrm{AD}$ and that targeting this adhesion molecule may have a therapeutic effect. Future studies are necessary to better understand the dynamics of immune cell trafficking in $\mathrm{AD}$ and TPLSM experiments that allow the imaging and analysis of leukocyte trafficking in the $\mathrm{AD}$ brain are discussed below.

\section{IMAGING THE CORTEX IN AD MICE}

In vivo imaging in the cortex of $\mathrm{AD}$ mice can be achieved using deeply anesthetized animals with the head fixed on a stereotaxic device to reduce movement artifacts. Mice in deep anesthesia cannot maintain their core body temperature at $35-38^{\circ} \mathrm{C}$. Heat lamps or a heated stage are therefore required to maintain the correct temperature because leukocyte motility is temperature dependent (70). An incision is made along the midline of the scalp to expose the skull, and any fascia overlying the skull is scraped away (71). Two different surgical preparations can be used for intravital TPLSM in the mouse cortex: the thinned skull preparation and open cranial window. Both these methods have advantages and drawbacks depending on the purpose of the investigation.

The thinned skull technique involves thinning the calvarium to approximately $20-30 \mu \mathrm{m}$ leaving an intact and almost transparent periosteal layer. A circular region of 1-2 $\mathrm{mm}$ in diameter is prepared with a high-speed micro drill and/or a stainless steel burr above the somatosensory and motor cortex. Heat and vibration artifacts are minimized during drilling by the frequent application of cold saline solution or artificial CSF. Heating is also avoided by interrupting the drilling every few seconds. Bone dust is removed using compressed air.

The mouse skull consists of two thin layers of compact bone, sandwiching a thick layer of spongy tissue. This spongy bone contains tiny cavities arranged in concentric circles and multiple canaliculi that carry blood vessels. After removing the external compact bone, the middle layer of spongy bone is carefully thinned to approximately $75 \%$ of its original thickness. Some bleeding from the blood vessels running through the canaliculi may occur during thinning but usually stops spontaneously (72). The clear visualization of the pial vasculature gives an indication of the skull thickness, then thinning is continued manually using a microsurgical blade. This process is repeated until the bone in the central region becomes flexible and maximum image clarity is achieved (Figures 1A,B). Once the surgical procedure is complete, the thinned skull preparation can be imaged immediately (73). When imaging is completed, the wound margins of the scalp are closed using a nylon suture.

Skull thinning creates a translucent viewing window allowing the visualization of cells up to a depth of 200-300 $\mu \mathrm{m}$ below the pial surface, including the meninges and neocortex but not the deeper brain structures (48). Imaging through the thinned cranial window is a minimally invasive method allowing long acquisition sessions because the thinned skull still protects the brain from external changes in temperature and pressure $(74,75)$. Despite minor bleeding from diploic vessels, the thinned skull technique leaves the majority of anastomoses between the diploic vessels and dural vessels intact. However, thinning the skull to a specific depth over a large zone is technically challenging due to the curvature of the skull, and the area should therefore be no more than $3 \mathrm{~mm}$ in diameter to avoid damage to the underlying tissue (72). Furthermore, in the case of multiple acquisition sessions over time, re-thinning the excess bone deposition after few days is necessary to prevent skull regrowth occluding the preparation (73). Among the two available techniques, the thinned cranial preparation is better for the analysis of larger structures, such as cerebral vasculature, amyloid plaques, and leukocytes $(19,51,52,76)$.

The study of AD mechanisms in mice may benefit from the creation of a small break with the tip of a needle in the lateral wall at the site of the thinned skull preparation to allow the delivery of fluorophores into the brain, leaving the thinned region intact. The hole is then filled with sterile bone wax, and the animals are allowed to recover on a heating pad before being returned to their cages. This approach can be used, e.g., to allow the diffusion of anti-A $\beta$ antibodies directly labeled with fluorescein or thioflavin $S$, a sensitive and specific fluorescent reporter for the dense-core subset of senile plaques. This molecule has been used to label A $\beta$ deposits in transgenic mouse models of amyloid deposition, and the growth rate of $A \beta$ plaques and cerebral $A \beta$ angiopathy have been extensively monitored in vivo (51, 55, 76-78).

The in vivo imaging of senile plaques in $\mathrm{AD}$ mice can also be achieved using Methoxy-X04, which can be administered intravenously or intraperitoneally (79). Methoxy-X04 is a relatively small, lipophilic molecule that can enter the brain rapidly and in sufficient amounts to allow the sensitive and specific detection of $\mathrm{A} \beta$ deposits (79). Senile plaques and cerebrovascular $\mathrm{A} \beta$ angiopathy in $\mathrm{AD}$-like mice are visible $30 \mathrm{~min}$ after intravenous injection or $24 \mathrm{~h}$ after intraperitoneal injection (79). The intravenous or intraperitoneal administration of Methoxy-X04 is a more physiological approach to label $\mathrm{A} \beta$ in vivo compared to the creation of a small break in the skull near the thinning region to allow delivery of fluorophores into the brain. 

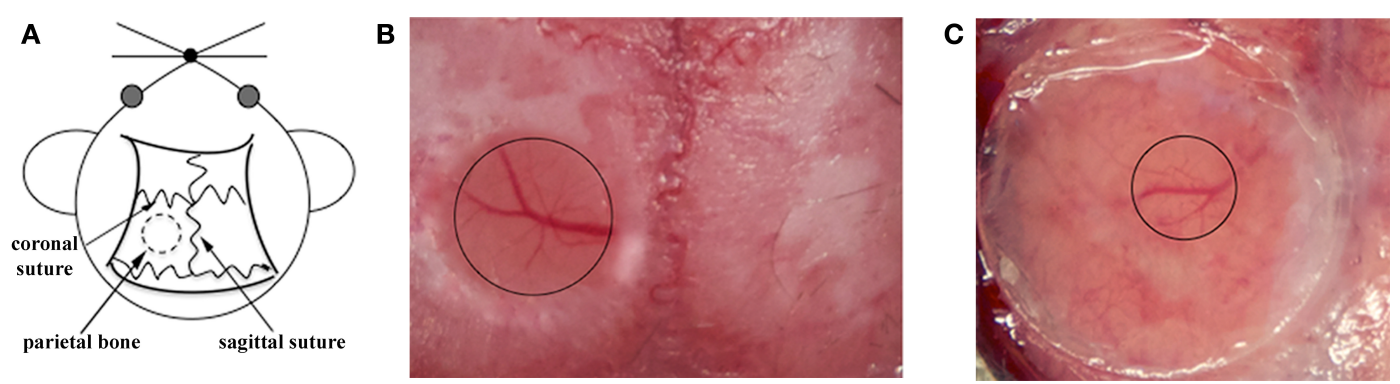

FIGURE 1 | Surgical procedures for TPLSM imaging of the brain cortex: thinned skull preparation and open cranial window. (A) For both procedures, a circular area of parietal bone is shaved with an electric razor and the scalp is sterilized with alcohol. The head of the anesthetized mouse is then fixed on a stereotaxic device to reduce movement artifacts during imaging, and the skull overlying the cortical region is exposed. (B) For the thinned skull preparation, a small region on the parietal zone of the skull is thinned using a high-speed micro drill and a stainless steel burr until the cortical vasculature can be seen clearly through the thinned skull. The thinned area is enclosed by the black circle. (C) For the open cranial window technique, a small island of cranial bone on the parietal region (black circle) is drilled, removed with a pair of sharp forceps, and covered with a circular glass coverslip.

The second major approach for TSPLM imaging is the open cranial window. The most challenging aspect of this technique is the surgical skill required for a successful preparation. In this procedure, a circular groove is drilled on the parietal region of the skull, and the island of cranial bone is carefully removed with a pair of sharp forceps. These should be held parallel to the skull surface as far as possible to avoid mechanical injury, because the dura can be attached to the overlying bone. Immediately after removing the region of the skull, slight bleeding above the dura may occur from small blood vessels attached to the removed skull fragment, but this should stop spontaneously within few seconds. The exposed brain tissue is preserved using a drop of $0.9 \% \mathrm{NaCl}$. A circular glass coverslip ( $5 \mathrm{~mm}$ in diameter) is placed over the incision site to cover the window (Figure 1C). After cementing the coverslip in place, mice are given a bolus of warm saline for rehydration and are allowed to recover from anesthesia for 7-14 days before imaging. The open cranial window technique also makes it unnecessary to introduce a needle-hole in the skull to deliver stains and labeling compounds. A recently described simple device provides easy physical access to the brain and allows long-term imaging (80). After the initial open cranial window surgery, a glass coverslip is applied as described above, but this has a pre-drilled access hole sealed with biocompatible silicone. This device allows many different types of manipulation to be carried out for weeks or months, e.g., drug, dye, and virus delivery, sample extraction, or electrophysiological recording and stimulation, while protecting the brain from infection (80).

The open cranial window provides improved optical access to the cortical layers, allowing repeated high-resolution imaging, and is preferable for the imaging of small structures, such as dendritic spines and filopodia $(73,81)$. However, removing the skull may induce a neuroinflammatory reaction and unavoidable meningeal vascular injury, involving the activation of microglia and astrocytes in the intact brain $(72,73,81,82)$. In this case, only cells lying deeper than $80 \mu \mathrm{m}$ below the pial surface should be considered for image analysis to eliminate possible artifacts caused by the surgical preparation.

To conduct multiple imaging sessions, some authors place a small metal bar containing a hole for a screw next to the coverslip to allow for repositioning of the mouse during subsequent imaging sessions. This approach has been used in Tg2576 mice for the long-term in vivo imaging to monitor individual amyloid plaques stained with Methoxy-X04 over a period of 6 weeks (55). Other studies using the same technique have followed individual $\mathrm{A} \beta$ plaques in Tg2576 mice for 5 months, confirming the biophysical model of $\mathrm{A} \beta$ plaque growth in vivo, which had been extrapolated from in vitro experiments.

Blood vessels can be labeled by the intravenous injection of fluorescent high-molecular-weight dextran $(>2000 \mathrm{kDa})$ or semiconductor nanocrystals (quantum dots), which suffer less interstitial leakage than dextrans (83) (Figure 2). Leukocytes can be labeled exvivo with vital dyes, such as 7-amino-4-chloromethylcoumarin (CMAC, blue), 5- (and 6-) carboxyfluorescein-diacetate succinimidyl-ester (CFSE, green), 5- (and 6-) (((4-chloromethyl) benzoyl)amino)tetramethylrhodamine (CMTMR, orange), $\mathrm{C}_{42} \mathrm{H}_{40} \mathrm{ClN}_{3} \mathrm{O}_{4}$ (CMTPX, red), and $\mathrm{C}_{33} \mathrm{H}_{24} \mathrm{~N}_{2} \mathrm{O}_{9}$ (SNARF-1, far red). The cells can then be adoptively transferred to syngeneic recipients allowing tracking for short time periods, usually a few days (Figure 2). Alternatively, the use of donor mice expressing fluorescent proteins ubiquitously is an option for the in vivo analysis of highly proliferative cell populations (84). Similarly, mice expressing fluorescent cell subsets allow the study of specific cell populations, such as neutrophils, lymphocytes, microglia, or macrophages $(61,85-87)$. Nowadays, mice engineered for the lineage-specific expression of GFP derivatives, such as eCFP and eYFP, or dsRed derivatives, such as tdTomato and mCherry, are widely used because they are less susceptible to phototoxicity and there is no need for cell isolation and labeling. However, most of these mice have been generated using GFP derivatives, limiting the ability to analyze several reporter genes in the same mouse simultaneously. Hence, new IR fluorescent dyes have been developed, which also facilitate deeper tissue imaging (88). The IR excitation wavelength requires the application of an optical parametric oscillator (OPO) to TPLSM. This allows live imaging for an extended duration because it reduces the photobleaching of fluorophores and phototoxicity-induced tissue damage.

The simultaneous expression of fluorescent proteins or staining using fluorescent dyes with different excitation spectra in the same 

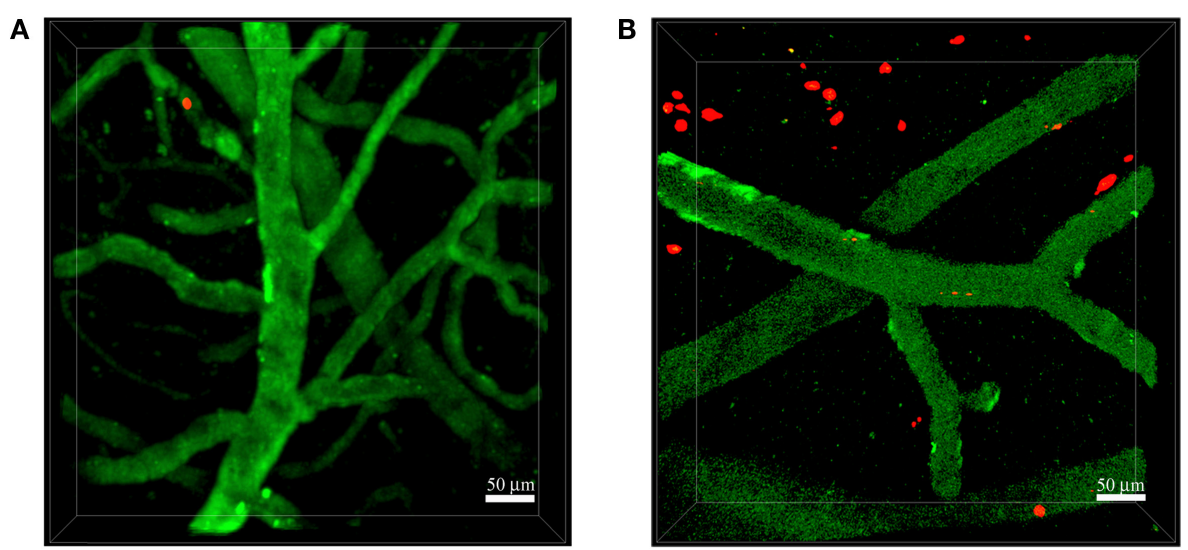

FIGURE 2 | Neutrophils invade the brain of 5xFAD mice. Representative TPLSM images of wild-type control mice (A) and 5xFAD mice (B) showing blood cortical vessels labeled in green using 525-nm non-targeted Qdots injected before image acquisition and neutrophils labeled in red with the fluorescent cell tracker CMTPX. The skull was exposed above the somatosensory cortex using the thinned skull preparation. We performed acquisition inside the brain parenchyma at a depth of approximately 150-250 $\mu \mathrm{m}$. Images were acquired 16-48 h after cell injection. (A) Neutrophils did not interact with the endothelium of blood vessels in wild-type control mice. (B) Numerous neutrophils migrated into the brain parenchyma of $5 x F A D$ mice. Scale bars $=50 \mu \mathrm{m}$.

mouse allows the in vivo visualization of interactions between specific cell populations and $\mathrm{A} \beta$ deposits, thus enabling the study of $\mathrm{AD}$-specific cellular dynamics in the brain. This approach was recently used by our group to demonstrate that neutrophils migrate inside the parenchyma of $5 \mathrm{xFAD}-\mathrm{YFPH}$ mice in areas with $\mathrm{A} \beta$ plaques labeled with Methoxy-X04, probably driven by chemotactic factors, potentially including A $\beta$ peptides (19) (Figure 3). Our TPLSM studies also showed that the extravasation of neutrophils inside the brain of $5 \mathrm{xFAD}-\mathrm{YFPH}$ mice occurs in areas depleted for eYFP fluorescent neurons (Figure 3). We speculate that eYFP fluorescent neurons are turned off in the zones with neutrophil infiltration, probably due to the cytotoxic effects of proinflammatory mediators released by neutrophils, such as ROS, enzymes, neutrophil extracellular traps (NETs), and cytokines. Alternatively, this may reflect an indirect negative effect caused by the activation of microglia. Moreover, we have shown that neutrophils display arrest and intraluminal crawling preferentially inside blood vessels with labeled $A \beta$ deposits and that some cells undergo diapedesis adjacent to vascular $\mathrm{A} \beta$ deposits (19). TPLSM experiments performed by others have shown that neutrophils are attracted inside the brain parenchyma by chronic $A \beta$ deposition - initially the cells move randomly outside the vessels and then they are suddenly and massively recruited to specific A $\beta$ plaques in the brain parenchyma (20).

Two-photon laser scanning microscopy can be used to study CNS-resident cells (such as neuronal subsets, astrocytes, microglia, and perivascular macrophages) in relation to each other or in relation to infiltrating leukocytes. TPLSM studies have shown that local resident microglia react to $\mathrm{A} \beta$ plaque formation by extending processes and subsequently migrating toward plaques in APP/PS1 mice crossed with mice containing fluorescent microglia (53). Furthermore, astrocytes in the mouse neocortex can be visualized in vivo by intravenous injection of the non-toxic molecule sulforhodamine B or by using transgenic mice, in which astrocytes express enhanced GFP under the control of the mouse glial fibrillary acidic protein (GFAP) promoter $(89,90)$. TPLSM in vivo imaging of astrocyte $\mathrm{Ca}^{2+}$ signaling revealed abnormalities in astrocyte activity that may contribute to vascular instability and thereby to neuronal cell death in several transgenic mouse models of AD (91). However, whether infiltrating leukocytes interact with resident neural cells is completely unknown in AD and future studies focusing on such interactions will be necessary to discover and understand these new disease mechanisms.

\section{IMAGE ACQUISITION AND ANALYSIS}

During image acquisition, mice are deeply anesthetized with isoflurane and the rate of respiration is controlled. The laser intensity and photomultiplier tube (PMT) gain need to be set carefully to minimize photodamage. The imaging volume and sampling frequency must be chosen to ensure successful image analysis with fine resolution in time and space. Stacks of optical sections are serially re-acquired at defined time intervals, and cell centroids need to be determined in order to track cell motion. The tracks locate the cells at each time point. They consist of serial sets of $x y z$ coordinates of single-cell centroids. The tracks are then exported as numerical data and are used to calculate specific parameters for the analysis of cell migration (36). Specialized software packages are used for automated 3D cell tracking and the analysis of migration paths for each cell. The automatic tracking and analysis of individual cells is more reliable when a small proportion of cells is labeled, because this reduces the likelihood of overlapping pixels representing different cells and allows the distinction of individual cells based on their centers of mass.

Several parameters can be used to analyze the migration behavior of cells during TPLSM experiments. Cell velocity can be represented as either instantaneous velocity or track velocity. Instantaneous velocity is a basic parameter derived from the displacement of the cell divided by the elapsed time (70). The track velocity is calculated from the median or mean instantaneous 
A

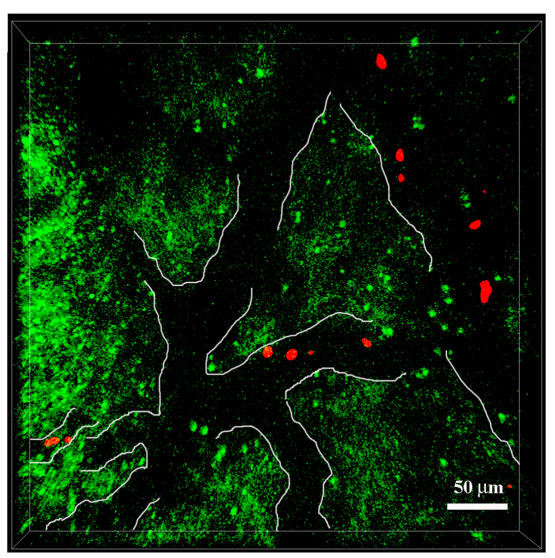

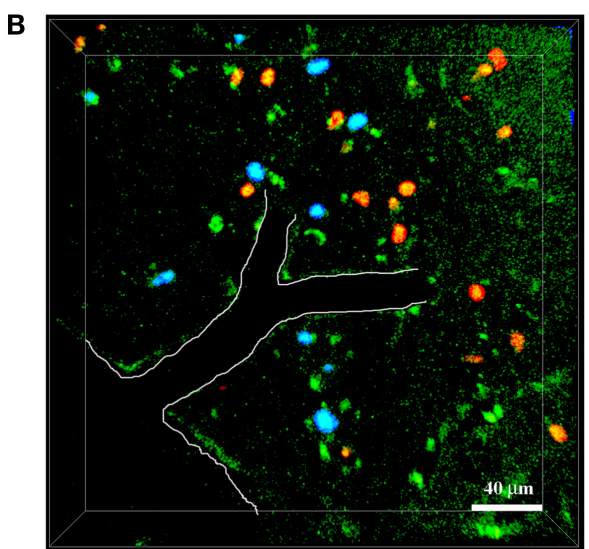

FIGURE 3 | Neutrophils infiltrate the brain of 5xFAD-YFPH mice in A $\beta$-rich areas. Representative TPLSM images of cortical regions in 5xFAD-YFPH mice showing YFP neurons in green, neutrophils in red stained with cell tracker CMTPX, and A $\beta$ plaques in blue labeled by the intravenous injection of Methoxy-X04. The vessel edges are traced artificially with a white line to show the vessel bed. (A) Neutrophils interact with the vascular endothelium. Scale bar $=50 \mu \mathrm{m}$. (B) Neutrophils infiltrate into the brain parenchyma, characterized by abundant A $\beta$ plaques and weak neuronal fluorescence. Scale bar $=40 \mu \mathrm{m}$.

velocity computed from all time intervals throughout a track, typically 6-14 time points at intervals of 20-50 s (92). Because leukocytes migrate along stromal cell networks, they do not travel along a linear path but instead make frequent turns in a "random walk." Therefore, higher sampling frequencies are necessary to provide an accurate readout of instantaneous velocities and to avoid the underestimation of real velocity when cells are persistent for only a finite period of time (93). It is easy to find differences in the motility parameters of leukocytes reported in previous studies, and such differences are likely to reflect the frame rate of data acquisition. If there is a long gap between each frame, then the true velocity of the cell will be underestimated. Therefore, the "fast sampling" theory (the use of sampling intervals shorter than the persistence time of the cell) should be taken into account in order to correctly compare data obtained in different laboratories (93).

A useful parameter that is derived from velocity is the arrest coefficient, representing the fraction of time during tracking in which a cell does not move (threshold $<2 \mu \mathrm{m} / \mathrm{min}$ ). It is calculated as the ratio of the time a cell is immotile over the whole observation time. The arrest coefficient is calculated from cell tracks and reported as the percentage of cells in the entire population (70). The arrest coefficient is high when leukocytes are in stable contact with other cells or when they swarm in a chemoattractant microenvironment (94).

The cell locomotion parameter allows some speculation on the nature of cell movement, e.g., cells may show directed migration along a gradient of a soluble or surface-immobilized chemoattractant. The displacement of a cell moving with a constant velocity is the shortest distance between the positions at two time points (95). An accurate parameter to analyze migration patterns is the mean displacement (MD) plot (70), in which the average displacement of a population of cells over specific time intervals is plotted against the square root of time. The slope of the resulting curve can be used to determine the motility coefficient of a cell population and measures the volume that a cell scans per unit time. Cells usually exhibit directed movement for a few minutes, which means that during short time intervals, they tend to move in one preferred direction (36).

The chemotactic index, also called the meandering index or straightness index, is another parameter representing the confinement of cell tracks and is calculated based on the ratio of displacement from origin by track length. The meandering can vary between 0 (the cell returns to its exact starting position) and 1 (a perfectly straight cell track). Cells exhibiting frequent angle changes will produce tracks with low meandering indices, whereas a meandering index of $0.7-1$ generally shows that cell migration has a strong directional bias (70).

During time-lapse acquisition, a moving cell is observed by taking snapshots at fixed time intervals; hence, the movement of each cell might be considered as a sequence of vectors. Trajectory vectors represent the direction of displacement of individual cells, so the calculation of angles between the direction of migration and various other directions in space is possible, e.g., the orientation of a blood vessel. The vector-vessel angles of individual tracks are useful for the description of tangential movement along an axis and are determined by mirroring the trajectory vectors onto the nearest vessel (reference vector), resulting in angles of $0-90^{\circ}$ for each cell track (96).

It is important to realize that cell populations analyzed using the parameters described above may consist of distinct subpopulations that have different biological roles and migration behaviors. These subpopulations may be revealed by plotting the distribution of the parameter of interest among all cells or by studying correlations between multiple motility parameters. For example, neutrophils invading the brain parenchyma in AD-like mice show two distinct behaviors: the first involves a strong directional bias characterized by a high meandering index, motility coefficient, and mean track velocity, whereas the second is characterized by low motility and undirected movement resembling swarming behavior (19). The undirected motility behavior with numerous cells displaying full arrest indicates the presence of activating stop signals for neutrophils in the brains of 
mice with AD-like pathology, whereas the directed movement of neutrophils strongly suggests the presence of chemotactic factors (19).

Other parameters calculated from leukocyte intratissue migration may reveal whether a chemoattractant released by injured tissue, resident cells, or recruited blood-derived leukocytes direct their movement. For example, after entering peripheral tissues, neutrophils assume an amoeboid motility profile characterized by coordinated migration along a chemokine gradient and cluster formation. The dramatic changes in neutrophil morphology can be analyzed by shape index (length/width), revealing the significant elongation of cell bodies, which is the characteristic of adhesion-independent movement associated with a low degree of cytoskeletal organization and a lack of discrete focal contacts (97).

The directional component of migration can be determined by measuring the turning angles of neutrophil tracks, which is achieved by calculating the angle change between vectors constructed from each time point. The peak angle distribution in absolute values varies in the range $0-180^{\circ}$, and changes of $10-30$ units indicate highly directed migration following a chemotactic gradient (97). In order to identify specific chemotactic molecules that direct leukocyte movement, instantaneous radial velocities can be calculated for the comparative chemotaxis analysis of wild-type and gene-deficient neutrophil populations (98). The radial velocity-time plot with fitted regression lines can provide insight into the recruitment dynamics of cell populations, and a reduction in radial velocity may be a consequence of impaired chemotaxis (98). Although no data are yet available in AD models, integrin-dependent intravascular neutrophil migration in sterile liver injuries requires CXCR2 ligands on liver sinusoids and formyl peptides in the injury zone (99). Furthermore, in sterile skin injuries, leukotriene B4 acts as an intercellular signal between neutrophils that allows rapid integrin-independent neutrophil recruitment throughout the tissue (98). Future studies are needed to characterize the dynamics of neutrophils as well as other leukocyte populations in the brains of $\mathrm{AD}$ models.

\section{FUTURE DIRECTIONS}

Two-photon laser scanning microscopy has made it possible to study leukocyte recruitment in the living brain, improving our understanding of immune cell functions in CNS diseases, such as $\mathrm{AD}$. Advanced imaging techniques will also allow us to define the role of leukocyte subpopulations at different stages of disease, as well as the relationships among migrating leukocytes, amyloid deposition, and tau pathology. Moreover, it is conceivable that TPLSM in the AD brain will help us to visualize and analyze the interplay between migrating leukocytes and resident cells, such as microglia, neurons, and astrocytes. TPLSM could also be used to unravel the role of toxic molecules, such as ROS and NO intermediates that may be produced by invading innate immunity cells during AD. For example, dihydroethidium and hydroethidine are cell-permeable fluorescent dyes suitable for the evaluation of ROS synthesis in vitro, but they have also been used successfully to visualize anion superoxide production in vivo $(100,101)$. The development of new fluorescent probes and the deep-tissue imaging capability of TPLSM may allow the in vivo visualization of ROS production by neural cells and infiltrating leukocytes.

One of the limitations of current imaging studies is the lack of access to deeper areas of the intact brain, such as the hippocampus, which is strongly affected during AD. Nevertheless, recently developed micro-optics and micro-mechanical components have improved deep-tissue imaging $(102,103)$. Indeed, the use of micro-prisms and gradient index (GRIN) lenses allows the longterm imaging of deep-layer cortical tissue in the mouse brain, although such devices must be inserted into the parenchyma, potentially altering neural cell functions and inducing inflammatory responses $(102,104)$. GRIN lenses feature plane optical surfaces and are focused by continuously changing the refractive index within the lens material by eliminating aberrations typically found in traditional spherical lenses. An alternative deep-tissue imaging method is stimulated emission depletion (STED) laser microscopy, which allows the exploration of deep areas, such as the hippocampus in living brains (105). This recent imaging technique improves spatial resolution by quenching the fluorescence everywhere except the central region, thus substantially reducing the size of the original fluorescence spot. Therefore, the higher peak signal-to-background ratio reveals more detailed structures, improving the quality of the images. In the near future, STED laser microscopy may expand to include the analysis of leukocyte recruitment in key brain areas during $\mathrm{AD}$, leading to the identification of new disease mechanisms. Moreover, STED technology brings in vivo super-resolution microscopy to small structures involved in the morphofunctional interactions between astrocytes and neurons, suggesting future exciting opportunities for the study of leukocyte interactions with neural cells (106).

Overall, the augmentation of TPLSM using optical systems, such as GRIN lenses or STED laser microscopy, and advances in fluorescent dye and protein engineering to produce brighter and more photostable fluorophores will play an important role in the study of molecular mechanisms controlling leukocytedependent brain injury and will help to identify new therapeutic approaches for $\mathrm{AD}$.

\section{ETHICS STATEMENT}

All mouse experiments were carried out in accordance with guidelines prescribed by the Ethics Committee for the usage of laboratory animals for research purposes at the University of Verona and by the Italian Ministry of Health.

\section{AUTHOR CONTRIBUTIONS}

All authors contributed equally.

\section{FUNDING}

This work was supported in part by the European Research Council grants 261079 NEUROTRAFFICKING and 693606 IMPEDE; National Multiple Sclerosis Society (NMSS), New York, NY, USA; Fondazione Cariverona; Italian Ministry of Health grant GR2009; Italian Ministry of Education and Research (MIUR); and Fondazione Italiana Sclerosi Multipla (FISM) (to GC). 


\section{REFERENCES}

1. Querfurth HW, LaFerla FM. Alzheimer's disease. N Engl J Med (2010) 362:329-44. doi:10.1056/NEJMra0909142

2. Wisniewski T, Goñi F. Immunotherapeutic approaches for Alzheimer's disease. Neuron (2015) 85:1162-76. doi:10.1016/j.neuron.2014.12.064

3. Heppner FL, Ransohoff RM, Becher B. Immune attack: the role of inflammation in Alzheimer disease. Nat Rev Neurosci (2015) 16:358-72. doi: $10.1038 / \mathrm{nrn} 3880.3$

4. Rossi B, Angiari S, Zenaro E, Budui SL, Constantin G. Vascular inflammation in central nervous system diseases: adhesion receptors controlling leukocyte-endothelial interactions. J Leukoc Biol (2011) 89:539-56. doi:10.1189/jlb.0710432

5. Ley K, Laudanna C, Cybulsky MI, Nourshargh S. Getting to the site of inflammation: the leukocyte adhesion cascade updated. Nat Rev Immunol (2007) 7:678-89. doi:10.1038/nri2156

6. El Khoury J, Toft M, Hickman SE, Means TK, Terada K, Geula C, et al. Ccr2 deficiency impairs microglial accumulation and accelerates progression of Alzheimer-like disease. Nat Med (2007) 13:432-8. doi:10.1038/nm1555

7. Simard AR, Soulet D, Gowing G, Julien JP, Rivest S. Bone marrow-derived microglia play a critical role in restricting senile plaque formation in Alzheimer's disease. Neuron (2006) 49:489-502. doi:10.1016/j. neuron.2006.01.022

8. Town T, Laouar Y, Pittenger C, Mori T, Szekely CA, Tan J, et al. Blocking TGFbeta-Smad $2 / 3$ innate immune signaling mitigates Alzheimer-like pathology. Nat Med (2008) 14:681-7. doi:10.1038/nm1781

9. Lebson L, Nash K, Kamath S, Herber D, Carty N, Lee DC, et al. Trafficking CD11b-positive blood cells deliver therapeutic genes to the brain of amyloid-depositing transgenic mice. J Neurosci (2010) 30:9651-8. doi:10.1523/ JNEUROSCI.0329-10.2010

10. Mildner A, Schmidt H, Nitsche M, Merkler D, Hanisch UK, Mack M, et al. Microglia in the adult brain arise from Ly-6ChiCCR2+ monocytes only under defined host conditions. Nat Neurosci (2007) 10:1544-53. doi:10.1038/ nn2015

11. Fiala M, Zhang L, Gan X, Sherry B, Taub D, Graves MC, et al. Amyloidbeta induces chemokine secretion and monocyte migration across a human blood-brain barrier model. Mol Med (1998) 4:480-9.

12. Giri R, Shen Y, Stins M, Du Yan S, Schmidt AM, Stern D, et al. Beta-amyloidinduced migration of monocytes across human brain endothelial cells involves RAGE and PECAM-1. Am J Physiol Cell Physiol (2000) 279:C1772-81.

13. Simard AR, Rivest $S$. Bone marrow stem cells have the ability to populate the entire central nervous system into fully differentiated parenchymal microglia. FASEB J (2004) 18:998-1000. doi:10.1096/f.04-1517fje

14. Jay TR, Miller CM, Cheng PJ, Graham LC, Bemiller S, Broihier ML, et al. TREM2 deficiency eliminates TREM2+ inflammatory macrophages and ameliorates pathology in Alzheimer's disease mouse models. J Exp Med (2015) 212:287-95. doi:10.1084/jem.20142322

15. Michaud JP, Bellavance MA, Préfontaine P, Rivest S. Real-time in vivo imaging reveals the ability of monocytes to clear vascular amyloid beta. Cell Rep (2013) 5:646-53. doi:10.1016/j.celrep.2013.10.010

16. Zarbock A, Ley K. Mechanisms and consequences of neutrophil interaction with the endothelium. Am J Pathol (2008) 172:1-7. doi:10.2353/ ajpath.2008.070502

17. DiStasi MR, Ley K. Opening the flood-gates: how neutrophil-endothelial interactions regulate permeability. Trends Immunol (2009) 30:547-56. doi:10.1016/j.it.2009.07.012

18. Fabene PF, Navarro Mora G, Martinello M, Rossi B, Merigo F, Ottoboni L, et al. A role for leukocyte-endothelial adhesion mechanisms in epilepsy. Nat Med (2008) 14:1377-83. doi:10.1038/nm.1878

19. Zenaro E, Pietronigro E, Della Bianca V, Piacentino G, Marongiu L, Budui S. Neutrophils promote Alzheimer's disease-like pathology and cognitive decline via LFA-1 integrin. Nat Med (2015) 21:880-6. doi:10.1038/nm.3913

20. Baik SH, Cha MY, Hyun YM, Cho H, Hamza B, Kim DK, et al. Migration of neutrophils targeting amyloid plaques in Alzheimer's disease mouse model. NeurobiolAging(2014)35:1286-92.doi:10.1016/j.neurobiolaging.2014.01.003

21. Joslin G, Griffin GL, August AM, Adams S, Fallon RJ, Senior RM, et al. The serpin-enzyme complex (SEC) receptor mediates the neutrophil chemotactic effect of alpha-1 antitrypsin-elastase complexes and amyloid-beta peptide. J Clin Invest (1992) 90:1150-4. doi:10.1172/JCI115934
22. Tiffany HL, Lavigne MC, Cui YH, Wang JM, Leto TL, Gao JL, et al. Amyloidbeta induces chemotaxis and oxidant stress by acting at formylpeptide receptor 2, a G protein-coupled receptor expressed in phagocytes and brain. J Biol Chem (2001) 276:23645-52. doi:10.1074/jbc.M101031200

23. Savage MJ, Iqbal M, Loh T, Trusko SP, Scott R, Siman R. Cathepsin G: localization in human cerebral cortex and generation of amyloidogenic fragments from the beta-amyloid precursor protein. Neuroscience (1994) 60:607-19. doi:10.1016/0306-4522(94)90490-1

24. Grammas P. A damaged microcirculation contributes to neuronal cell death in Alzheimer's disease. Neurobiol Aging (2000) 21:199-205. doi:10.1016/ S0197-4580(00)00102-0

25. Brock AJ, Kasus-Jacobi A, Lerner M, Logan S, Adesina AM, Anne Pereira $\mathrm{H}$. The antimicrobial protein, CAP37, is upregulated in pyramidal neurons during Alzheimer's disease. Histochem Cell Biol (2015) 144:293-308. doi:10.1007/s00418-015-1347-x

26. Itagaki S, McGeer PL, Akiyama H. Presence of T-cytotoxic suppressor and leucocyte common antigen positive cells in Alzheimer's disease brain tissue. Neurosci Lett (1988) 91:259-64. doi:10.1016/0304-3940(88)90690-8

27. Rogers J, Luber-Narod J, Styren SD, Civin WH. Expression of immune system-associated antigens by cells of the human central nervous system: relationship to the pathology of Alzheimer's disease. Neurobiol Aging (1988) 9:339-49. doi:10.1016/S0197-4580(88)80079-4

28. Togo T, Akiyama H, Iseki E, Kondo H, Ikeda K, Kato M, et al. Occurrence of T cells in the brain of Alzheimer's disease and other neurological diseases. J Neuroimmunol (2002) 124:83-92. doi:10.1016/S0165-5728(01)00496-9

29. Town T, Tan J, Flavell RA, Mullan M. T-cells in Alzheimer's disease. Neuromol Med (2005) 7:255-64. doi:10.1385/NMM:7:3:255

30. Lueg G, Gross CC, Lohmann H, Johnen A, Kemmling A, Deppe M, et al. Clinical relevance of specific T-cell activation in the blood and cerebrospinal fluid of patients with mild Alzheimer's disease. Neurobiol Aging (2015) 36:81-9. doi:10.1016/j.neurobiolaging.2014.08.008

31. Browne TC, McQuillan K, McManus RM, O’Reilly JA, Mills KH, Lynch MA. IFN- $\gamma$ production by amyloid $\beta$-specific Th1 cells promotes microglial activation and increases plaque burden in a mouse model of Alzheimer's disease. J Immunol (2013) 190:2241-51. doi:10.4049/jimmunol.1200947

32. Denk W, Strickler JH, Webb WW. Two-photon laser scanning fluorescence microscopy. Science (1990) 248:73-6. doi:10.1126/science.2321027

33. Helmchen F. Chapter 2. Two-photon functional imaging of neuronal activity. In: Frostig RD, editor. In Vivo Optical Imaging of Brain Function. 2nd ed. Boca Raton, FL: CRC Press; Taylor \& Francis (2009). p. 37-58.

34. Helmchen F, Denk W. Deep tissue two-photon microscopy. Nat Methods (2005) 2:932-40. doi:10.1038/nmeth818

35. Svoboda K, Yasuda R. Principles of two-photon excitation microscopy and its applications to neuroscience. Neuron (2006) 50:823-39. doi:10.1016/j. neuron.2006.05.019

36. Sumen C, Mempel TR, Mazo IB, von Andrian UH. Intravital microscopy: visualizing immunity in context. Immunity (2004) 21:315-29. doi:10.1016/j. immuni.2004.08.006

37. Henrickson SE, von Andrian UH. Single-cell dynamics of T-cell priming. Curr Opin Immunol (2007) 19:249-58. doi:10.1016/j.coi.2007.04.013

38. Germain RN, Robey EA, Cahalan MD. A decade of imaging cellular motility and interaction dynamics in the immune system. Science (2012) 336:1676-81. doi:10.1126/science. 1221063

39. Balagopalan L, Sherman E, Barr VA, Samelson LE. Imaging techniques for assaying lymphocyte activation in action. Nat Rev Immunol (2011) 11:21-33. doi: $10.1038 /$ nri2903

40. Kolaczkowska E, Kubes P. Neutrophil recruitment and function in health and inflammation. Nat Rev Immunol (2013) 13:159-75. doi:10.1038/nri3399

41. Phillipson M, Kubes P. The neutrophil in vascular inflammation. Nat Med (2011) 17:1381-90. doi:10.1038/nm.2514

42. Grutzendler J, Gan WB. Two-photon imaging of synaptic plasticity and pathology in the living mouse brain. NeuroRx (2006) 3:489-96. doi:10.1016/j. nurx.2006.07.005

43. Grutzendler J, Helmin K, Tsai J, Gan WB. Various dendritic abnormalities are associated with fibrillar amyloid deposits in Alzheimer's disease. Ann $N$ Y Acad Sci (2007) 1097:30-9. doi:10.1196/annals.1379.003

44. Bhatt DH, Zhang S, Gan WB. Dendritic spine dynamics. Annu Rev Physiol (2009) 71:261-82. doi:10.1146/annurev.physiol.010908.163140 
45. Nayak D, Zinselmeyer BH, Corps KN, McGavern DB. In vivo dynamics of innate immune sentinels in the CNS. Intravital (2012) 1:95-106. doi:10.4161/ intv. 22823

46. Trachtenberg JT, Chen BE, Knott GW, Feng G, Sanes JR, Welker E, et al. Long-term in vivo imaging of experience-dependent synaptic plasticity in adult cortex. Nature (2002) 420:788-94. doi:10.1038/nature01273

47. Chklovskii DB, Mel BW, Svoboda K. Cortical rewiring and information storage. Nature (2004) 431:782-8. doi:10.1038/nature03012

48. Nimmerjahn A, Kirchhoff F, Helmchen F. Resting microglial cells are highly dynamic surveillants of brain parenchyma in vivo. Science (2005) 308:1314-8. doi:10.1126/science.1110647

49. Grienberger C, Konnerth A. Imaging calcium in neurons. Neuron (2012) 73:862-85. doi:10.1016/j.neuron.2012.02.011

50. Stosiek C, Garaschuk O, Holthoff K, Konnerth A. In vivo two-photon calcium imaging of neuronal networks. Proc Natl Acad Sci U S A (2003) 100:7319-24. doi:10.1073/pnas.1232232100

51. Christie RH, Bacskai BJ, Zipfel WR, Williams RM, Kajdasz ST, Webb WW, et al. Growth arrest of individual senile plaques in a model of Alzheimer's disease observed by in vivo multiphoton microscopy. JNeurosci (2001) 21:858-64.

52. Tsai J, Grutzendler J, Duff K, Gan WB. Fibrillar amyloid deposition leads to local synaptic abnormalities and breakage of neuronal branches. Nat Neurosci (2004) 7:1181-3. doi:10.1038/nn1335

53. Bolmont T, Haiss F, Eicke D, Radde R, Mathis CA, Klunk WE, et al. Dynamics of the microglial/amyloid interaction indicate a role in plaque maintenance. J Neurosci (2008) 28:4283-92. doi:10.1523/JNEUROSCI.4814-07.2008

54. Bittner T, Fuhrmann M, Burgold S, Ochs SM, Hoffmann N, Mitteregger $\mathrm{G}$, et al. Multiple events lead to dendritic spine loss in triple transgenic Alzheimer's disease mice. PLoS One (2010) 5:e15477. doi:10.1371/journal. pone. 0015477

55. Burgold S, Bittner T, Dorostkar MM, Kieser D, Fuhrmann M, Mitteregger G, et al. In vivo multiphoton imaging reveals gradual growth of newborn amyloid plaques over weeks. Acta Neuropathol (2011) 121:327-35. doi:10.1007/ s00401-010-0787-6

56. Hefendehl JK, Wegenast-Braun BM, Liebig C, Eicke D, Milford D, Calhoun $\mathrm{ME}$, et al. Long-term in vivo imaging of beta-amyloid plaque appearance and growth in a mouse model of cerebral beta-amyloidosis. J Neurosci (2011) 31:624-9. doi:10.1523/JNEUROSCI.5147-10.2011

57. Burgold S, Filser S, Dorostkar MM, Schmidt B, Herms J. In vivo imaging reveals sigmoidal growth kinetic of $\beta$-amyloid plaques. Acta Neuropathol Commun (2014) 2:30. doi:10.1186/2051-5960-2-30

58. Spires TL, Meyer-Luehmann M, Stern EA. Dendritic spine abnormalities in amyloid precursor protein transgenic mice demonstrated by gene transfer and intravital multiphoton microscopy. JNeurosci (2005) 25:7278-87. doi:10.1523/JNEUROSCI.1879-05.2005

59. Bittner T, Burgold S, Dorostkar MM, Fuhrmann M, Wegenast-Braun BM, Schmidt B, et al. Amyloid plaque formation precedes dendritic spine loss. Acta Neuropathol (2012) 124:797-807. doi:10.1007/s00401-012-1047-8

60. Brendza RP, Bacskai BJ, Cirrito JR, Simmons KA, Skoch JM, Klunk WE, et al. Anti-Abeta antibody treatment promotes the rapid recovery of amyloid-associated neuritic dystrophy in PDAPP transgenic mice. J Clin Invest (2005) 115:428-33. doi:10.1172/JCI23269

61. Jung S, Aliberti J, Graemmel P, Sunshine MJ, Kreutzberg GW, Sher A, et al. Analysis of fractalkine receptor CX(3)CR1 function by targeted deletion and green fluorescent protein reporter gene insertion. Mol Cell Biol (2000) 20:4106-14. doi:10.1128/MCB.20.11.4106-4114.2000

62. Zuo Y, Lubischer JL, Kang H, Tian L, Mikesh M, Marks A, et al. Fluorescent proteins expressed in mouse transgenic lines mark subsets of glia, neurons, macrophages, and dendritic cells for vital examination. JNeurosci (2004) 24:10999-1009. doi:10.1523/JNEUROSCI.3934-04.2004

63. Meyer-Luehmann M, Spires-Jones TL, Prada C, Garcia-Alloza M, de Calignon A, Rozkalne A, et al. Rapid appearance and local toxicity of amyloid-beta plaques in a mouse model of Alzheimer's disease. Nature (2008) 451:720-4. doi:10.1038/nature06616

64. Krabbe G, Halle A, Matyash V, Rinnenthal JL, Eom GD, Bernhardt U, et al. Functional impairment of microglia coincides with beta-amyloid deposition in mice with Alzheimer-like pathology. PLoS One (2013) 8:e60921. doi:10.1371/journal.pone.0060921
65. Mrass $\mathrm{P}$, Weninger W. Immune cell migration as a means to control immune privilege: lessons from the CNS and tumors. Immunol Rev (2006) 213:195-212. doi:10.1111/j.1600-065X.2006.00433.x

66. Bartholomäus I, Kawakami N, Odoardi F, Schläger C, Miljkovic D, Ellwart JW, et al. Effector T cell interactions with meningeal vascular structures in nascent autoimmune CNS lesions. Nature (2009) 462:94-8. doi:10.1038/ nature 08478

67. Coombes JL, Robey EA. Dynamic imaging of host-pathogen interactions in vivo. Nat Rev Immunol (2010) 10:353-64. doi:10.1038/nri2746

68. Kawakami N, Flügel A. Knocking at the brain's door: intravital two-photon imaging of autoreactive T cell interactions with CNS structures. Semin Immunopathol (2010) 32:275-87. doi:10.1007/s00281-010-0216-x

69. McGavern DB, Kang SS. Illuminating viral infections in the nervous system. Nat Rev Immunol (2011) 11:318-29. doi:10.1038/nri2971

70. Zinselmeyer BH, Dempster J, Wokosin DL, Cannon JJ, Pless R, Parker I, et al. Chapter 16. Two-photon microscopy and multidimensional analysis of cell dynamics. Methods Enzymol (2009) 461:349-78. doi:10.1016/ S0076-6879(09)05416-0

71. Zenaro E, Rossi B, Angiari S, Constantin G. Use of imaging to study leukocyte trafficking in the central nervous system. Immunol Cell Biol (2013) 91:271-80. doi:10.1038/icb.2012.81

72. Xu HT, Pan F, Yang G, Gan WB. Choice of cranial window type for in vivo imaging affects dendritic spine turnover in the cortex. Nat Neurosci (2007) 10:549-51. doi:10.1038/nn1883

73. Yang G, Pan F, Parkhurst CN, Grutzendler J, Gan WB. Thinned-skull cranial window technique for long-term imaging of the cortex in live mice. Nat Protoc (2010) 5:201-8. doi:10.1038/nprot.2009.222

74. Kim JV, Jiang N, Tadokoro CE, Liu L, Ransohoff RM, Lafaille JJ, et al. Two-photon laser scanning microscopy imaging of intact spinal cord and cerebral cortex reveals requirement for CXCR6 and neuroinflammation in immune cell infiltration of cortical injury sites. J Immunol Methods (2010) 352:89-100. doi:10.1016/j.jim.2009.09.007

75. Davalos D, Lee JK, Smith WB, Brinkman B, Ellisman MH, Zheng B, et al. Stable in vivo imaging of densely populated glia, axons and blood vessels in the mouse spinal cord using two-photon microscopy. J Neurosci Methods (2008) 169:1-7. doi:10.1016/j.jneumeth.2007.11.011

76. Yoder EJ, Kleinfeld D. Cortical imaging through the intact mouse skull using two-photon excitation laser scanning microscopy. Microsc Res Tech (2002) 56:304-5. doi:10.1002/jemt.10002

77. D’Amore JD, Kajdasz ST, McLellan ME, Bacskai BJ, Stern EA, Hyman BT. In vivo multiphoton imaging of a transgenic mouse model of Alzheimer disease reveals marked thioflavine-S-associated alterations in neurite trajectories. J Neuropathol Exp Neurol (2003) 62:137-45. doi:10.1093/jnen/62.2.137

78. Dong J, Revilla-Sanchez R, Moss S, Haydon PG. Multiphoton in vivo imaging of amyloid in animal models of Alzheimer's disease. Neuropharmacology (2010) 59:268-75. doi:10.1016/j.neuropharm.2010.04.007

79. Klunk WE, Bacskai BJ, Mathis CA, Kajdasz ST, McLellan ME, Frosch MP, et al. Imaging Abeta plaques in living transgenic mice with multiphoton microscopy and methoxy-X04, a systemically administered congo red derivative. J Neuropathol Exp Neurol (2002) 61:797-805. doi:10.1093/jnen/61.9.797

80. Roome CJ, Kuhn B. Chronic cranial window with access port for repeated cellular manipulations, drug application, and electrophysiology. Front Cell Neurosci (2014) 8:379. doi:10.3389/fncel.2014.00379

81. Majewska AK, Newton JR, Sur M. Remodeling of synaptic structure in sensory cortical areas in vivo. J Neurosci (2006) 26:3021-9. doi:10.1523/ JNEUROSCI.4454-05.2006

82. Roth TL, Nayak D, Atanasijevic T, Koretsky AP, Latour LL, McGavern DB. Transcranial amelioration of inflammation and cell death after brain injury. Nature (2014) 505:223-8. doi:10.1038/nature12808

83. Stroh M, Zimmer JP, Duda DG, Levchenko TS, Cohen KS, Brown EB, et al. Quantum dots spectrally distinguish multiple species within the tumor milieu in vivo. Nat Med (2005) 11:678-82. doi:10.1038/nm1247

84. Luche H, Weber O, Nageswara Rao T, Blum C, Fehling HJ. Faithful activation of an extra-bright red fluorescent protein in "knock-in" Cre-reporter mice ideally suited for lineage tracing studies. Eur J Immunol (2007) 37:43-53. doi:10.1002/eji.200636745

85. Manfra DJ, Chen SC, Yang TY, Sullivan L, Wiekowski MT, Abbondanzo S, et al. Leukocytes expressing green fluorescent protein as novel reagents for 
adoptive cell transfer and bone marrow transplantation studies. Am J Pathol (2001) 158:41-7. doi:10.1016/S0002-9440(10)63942-9

86. Hasenberg A, Hasenberg M, Männ L, Neumann F, Borkenstein L, Stecher M, et al. Catchup: a mouse model for imaging-based tracking and modulation of neutrophil granulocytes. Nat Methods (2015) 12:445-52. doi:10.1038/ nmeth. 3322

87. Sasmono RT, Oceandy D, Pollard JW, Tong W, Pavli P, Wainwright BJ, et al. A macrophage colony-stimulating factor receptor-green fluorescent protein transgene is expressed throughout the mononuclear phagocyte system of the mouse. Blood (2003) 101:1155-63. doi:10.1182/blood-2002-02-0569

88. Swirski FK, Berger CR, Figueiredo JL, Mempel TR, von Andrian UH, Pittet $\mathrm{MJ}$, et al. A near-infrared cell tracker reagent for multiscopic in vivo imaging and quantification of leukocyte immune responses. PLoS One (2007) 2:e1075. doi:10.1371/journal.pone.0001075

89. Appaix F, Girod S, Boisseau S, Römer J, Vial JC, Albrieux M, et al. Specific in vivo staining of astrocytes in the whole brain after intravenous injection of sulforhodamine dyes. PLoS One (2012) 7:e35169. doi:10.1371/journal.pone.0035169

90. Suzuki R, Watanabe J, Arata S, Funahashi H, Kikuyama S, Shioda S. A transgenic mouse model for the detailed morphological study of astrocytes. Neurosci Res (2003) 47:451-4. doi:10.1016/j.neures.2003.08.008

91. Takano T, Han X, Deane R, Zlokovic B, Nedergaard M. Two-photon imaging of astrocytic $\mathrm{Ca} 2+$ signaling and the microvasculature in experimental mice models of Alzheimer's disease. Ann N Y Acad Sci (2007) 1097:40-50. doi:10.1196/annals.1379.004

92. Aoshi T, Zinselmeyer BH, Konjufca V, Lynch JN, Zhang X, Koide Y, et al. Bacterial entry to the splenic white pulp initiates antigen presentation to CD8+ T cells. Immunity (2008) 29:476-86. doi:10.1016/j. immuni.2008.06.013

93. Bullen A, Friedman RS, Krummel MF. Two-photon imaging of the immune system: a custom technology platform for high-speed, multicolor tissue imaging of immune responses. Curr Top Microbiol Immunol (2009) 334:1-29. doi:10.1007/978-3-540-93864-4_1

94. Tadokoro CE, Shakhar G, Shen S, Ding Y, Lino AC, Maraver A, et al. Regulatory $\mathrm{T}$ cells inhibit stable contacts between CD4+ T cells and dendritic cells in vivo. J Exp Med (2006) 203:505-11. doi:10.1084/ jem.20050783

95. Wei SH, Parker I, Miller MJ, Cahalan MD. A stochastic view of lymphocyte motility and trafficking within the lymph node. Immunol Rev (2003) 195:136-59. doi:10.1034/j.1600-065X.2003.00076.x

96. Siffrin V, Brandt AU, Radbruch H, Herz J, Boldakowa N, Leuenberger $\mathrm{T}$, et al. Differential immune cell dynamics in the CNS cause CD4+ T cell compartmentalization. Brain (2009) 132:1247-58. doi:10.1093/ brain/awn354
97. Graham DB, Zinselmeyer BH, Mascarenhas F, Delgado R, Miller MJ, Swat W. ITAM signaling by Vav family Rho guanine nucleotide exchange factors regulates interstitial transit rates of neutrophils in vivo. PLoS One (2009) 4:e4652. doi:10.1371/journal.pone.0004652

98. Lämmermann T, Afonso PV, Angermann BR, Wang JM, Kastenmüller W, Parent CA, et al. Neutrophil swarms require LTB4 and integrins at sites of cell death in vivo. Nature (2013) 498:371-5. doi:10.1038/nature12175

99. McDonald B, Pittman K, Menezes GB, Hirota SA, Slaba I, Waterhouse CC, et al. Intravascular danger signals guide neutrophils to sites of sterile inflammation. Science (2010) 330:362-6. doi:10.1126/science.1195491

100. Hanson KM, Clegg RM. Two-photon fluorescence imaging and reactive oxygen species detection within the epidermis. Methods Mol Biol (2005) 289:413-22.

101. Wang X, Fang H, Huang Z, Shang W, Hou T, Cheng A, et al. Imaging ROS signaling in cells and animals. J Mol Med (Berl) (2013) 91:917-27. doi:10.1007/ s00109-013-1067-4

102. Andermann ML, Gilfoy NB, Goldey GJ, Sachdev RN, Wölfel M, McCormick DA, et al. Chronic cellular imaging of entire cortical columns in awake mice using microprisms. Neuron (2013) 80:900-13. doi:10.1016/j. neuron.2013.07.052

103. Wang BG, König K, Halbhuber KJ. Two-photon microscopy of deep intravital tissues and its merits in clinical research. J Microsc (2010) 238:1-20. doi:10.1111/j.1365-2818.2009.03330.x

104. Barretto RP, Ko TH, Jung JC, Wang TJ, Capps G, Waters AC, et al. Timelapse imaging of disease progression in deep brain areas using fluorescence microendoscopy. Nat Med (2011) 17:223-8. doi:10.1038/nm.2292

105. Kawakami R, Sawada K, Kusama Y, Fang YC, Kanazawa S, Kozawa Y, et al. In vivo two-photon imaging of mouse hippocampal neurons in dentate gyrus using a light source based on a high-peak power gain-switched laser diode. Biomed Opt Express (2015) 6:891-901. doi:10.1364/BOE.6.000891

106. Panatier A, Arizono M, Nägerl UV. Dissecting tripartite synapses with STED microscopy. Philos Trans R Soc Lond B Biol Sci (2014) 369:20130597. doi:10.1098/rstb.2013.0597

Conflict of Interest Statement: The authors declare that the research was conducted in the absence of any commercial or financial relationships that could be construed as a potential conflict of interest.

Copyright $\odot 2016$ Pietronigro, Zenaro and Constantin. This is an open-access article distributed under the terms of the Creative Commons Attribution License (CC BY). The use, distribution or reproduction in other forums is permitted, provided the original author(s) or licensor are credited and that the original publication in this journal is cited, in accordance with accepted academic practice. No use, distribution or reproduction is permitted which does not comply with these terms. 
OPEN ACCESS

Edited by:

Saparna Pai,

The Centenary Institute, Australia

Reviewed by:

Nathan Karin,

Technion - Israel Institute of

Technology, Israel

Anne Kathrin Mausberg,

Heinrich Heine University, Germany

*Correspondence:

Helena Radbruch

helena.radbruch@charite.de;

Raluca Niesner niesner@drfz.de

Specialty section:

This article was submitted to Multiple Sclerosis and Neuroimmunology,

a section of the journal

Frontiers in Immunology

Received: 17 October 2015 Accepted: 25 February 2016

Published: 14 March 2016

Citation:

Radbruch H, Bremer D, Guenther R,

Cseresnyes Z, Lindquist $R$,

Hauser AE and Niesner $R$ (2016)

Ongoing Oxidative Stress Causes

Subclinical Neuronal Dysfunction in the Recovery Phase of EAE.

Front. Immunol. 7:92.

doi: 10.3389/fimmu.2016.00092

\section{Ongoing Oxidative Stress Causes Subclinical Neuronal Dysfunction in the Recovery Phase of EAE}

\author{
Helena Radbruch ${ }^{*}$, Daniel Bremer ${ }^{2}$, Robert Guenther ${ }^{2}$, Zoltan Cseresnyes ${ }^{2}$, \\ Randall Lindquist ${ }^{2}$, Anja E. Hauser ${ }^{2,3}$ and Raluca Niesner ${ }^{2 *}$
}

${ }^{1}$ Department of Neuropathology, Charité - Universitätsmedizin Berlin, Berlin, Germany, ${ }^{2}$ German Rheumatism Research Center (DRFZ) a Leibniz Institute, Berlin, Germany, ${ }^{3}$ Immundynamics, Charité - Universiätsmedizin Berlin, Berlin, Germany

Most multiple sclerosis (MS) patients develop over time a secondary progressive disease course, characterized histologically by axonal loss and atrophy. In early phases of the disease, focal inflammatory demyelination leads to functional impairment, but the mechanism of chronic progression in MS is still under debate. Reactive oxygen species generated by invading and resident central nervous system (CNS) macrophages have been implicated in mediating demyelination and axonal damage, but demyelination and neurodegeneration proceed even in the absence of obvious immune cell infiltration, during clinical recovery in chronic MS. Here, we employ intravital NAD(P)H fluorescence lifetime imaging to detect functional NADPH oxidases (NOX1-4, DUOX1, 2) and, thus, to identify the cellular source of oxidative stress in the CNS of mice affected by experimental autoimmune encephalomyelitis (EAE) in the remission phase of the disease. This directly affects neuronal function in vivo, as monitored by cellular calcium levels using intravital FRET-FLIM, providing a possible mechanism of disease progression in MS.

Keywords: NOX, EAE/MS, intravital imaging, FLIM-FRET, calcium

\section{INTRODUCTION}

Multiple sclerosis (MS) is a chronic neuroinflammatory disease, with most patients exhibiting a relapsing and remitting course of disease. The neurological damage is a consequence of a mainly T cell-driven immune reaction against myelin in the central nervous system (CNS) (1). Macrophages/ microglia, $\mathrm{B}$, and $\mathrm{T}$ cells create an acute inflammatory setting, resulting in demyelination and neuronal damage. Most of the patients who experience a second episode develop further relapses. Despite the intensive analysis of the acute immune attack, only little is known about the processes going on at the lesion site after the initial insult $(2,3)$.

Why and where do new relapses appear? What factors determine the chronicity of a lesion and the course of disease? "Old" lesions appear morphologically inert and are characterized by single perivascular $\mathrm{T}$ cells, minimal axonal damage in histological stainings with anti-amyloid precursor protein (APP) antibodies and a dominant fibrotic glial scar $(4,5)$. In contrast to the progressive disease phase, the inflammatory phase is well modeled by murine experimental autoimmune encephalomyelitis (EAE). In this mouse model using an immunization with $\mathrm{MOG}_{35-55}$ peptide, acute clinical signs remit after a few days and mice enter into a chronic phase with or without a residuum of neurological deficits (1). Reactive oxygen species (ROS) generated by invading and resident CNS macrophages have been implicated in mediating demyelination and axonal damage (6-8). In this 
study, we address implications of the ceased immune attack for the CNS tissue, beyond the fact that the majority of peripheral immune cells disappeared. In MS patients, we previously detected an ongoing over-activation of NADPH oxidases (especially NOX2) in blood monocytes during remission (8). Using intravital $\mathrm{NAD}(\mathrm{P}) \mathrm{H}$ fluorescence lifetime imaging in mice affected by EAE, during the inflammatory phase (onset and peak of the disease), we detected a massively increased amount of functional NADPH oxidases (NOX1-4, DUOX1, 2) within the CNS as compared to healthy controls (8). Using the same method, we investigated whether functional NADPH oxidases are still present in the CNS after recovery of EAE and, thus, whether oxidative stress is still ongoing in absence of peripheral infiltration of the CNS. We simultaneously monitor calcium concentrations in neurons using intravital FRET-FLIM-based neuronal calcium imaging to evaluate the reaction of the neurons on the altered CNS environment over the course of EAE development and remission. Thereby, we investigate whether in mice with clinical recovery morphologically inert appearing lesions exhibit residual inflammation, as reflected by increased oxidative stress and sub-clinical neuronal dysfunction, in order to better understand mechanisms of chronicity and disease progression in MS and related diseases.

\section{RESULTS}

\section{Characterization of the Remission Phase in the CNS of Mice Affected by EAE}

The grade of inflammation in brain stem of mice with EAE after clinical recovery (remission) was characterized and compared to animals at the peak of disease and to healthy controls. Our aim was to first characterize peripheral and CNS resident cellular compartments during the remission phase by means of intravital imaging and to corroborate previous results concerning the lack of overt inflammation in this phase.

\section{Characterization of Cellular Markers in the CNS, during EAE Remission}

It is widely accepted that in MS, inactive CNS lesions with no signs of immune infiltration are detectable. In our EAE model, some mice show a complete clinical recovery of EAE signs. We characterized these mice by FACS analysis of whole murine CNS (brain and spinal cord) and demonstrated that both monocytes/ macrophages $\left(\mathrm{CD} 45^{\text {high }} \mathrm{CD} 11 \mathrm{~b}^{+}\right.$cells) and $\mathrm{T}$ cells $\left(\mathrm{CD} 45^{\text {high }} \mathrm{CD}^{+}\right.$ cells) disappear from the CNS during the remission phase of EAE. Only $7.9 \pm 2.8 \%$ of the isolated CNS cells were CD $45^{\text {high }} \mathrm{CD} 11 \mathrm{~b}^{+}$ cells (macrophages/monocytes), comparable with healthy controls with $6.2 \pm 2.4 \%$ (Figures 1A,B), whereas their frequency during onset and peak of EAE was previously shown to be strongly increased, to $\sim 50 \%$ of the infiltrates $(8-10)$. The majority of cells after EAE recovery were cells with characteristics of microglia: $72.5 \pm 3.6 \%$ were $\mathrm{CD} 45^{\text {low }} \mathrm{CD} 11 \mathrm{~b}^{+}$of which $95.2 \pm 6.7 \%$ expressed CX3CR1. The overlap of CX3CR1 and tdRFP (LysM) was in both compartments under $5 \%\left(3.5 \pm 3.2 \%\right.$ for $\mathrm{CD}_{4} 5^{\text {high }} \mathrm{CD} 11 \mathrm{~b}^{+}$ cells and $3.5 \pm 3.1 \%$ for $\mathrm{CD} 45^{\text {low }} \mathrm{CD} 11 \mathrm{~b}^{+}$cells). $\mathrm{CD} 45^{\text {high }} \mathrm{CD}^{+}$ cells - typically present during the peak of EAE (11) - mainly disappeared after EAE recovery, constituting only $0.2 \pm 0.1 \%$ of total cell number. All these findings are in line with the low clinical scores of the mice (between 0 and 0.5 ; Table 1) and are consistent with previous observations of cellular compositions after EAE recovery (10). Our results encompass two independent EAE experiments with a total number of $n=3$ mice analyzed in remission phase (Table 1 ) and $n=5$ analyzed healthy mice.

Using intravital microscopy in CX3CR $1^{+/-}$EGFP mice $(n=3)$ after EAE recovery, we could show a reduced overlap of $3.6 \pm 1.8 \%$ between EGFP and i.v. injected sulforhodamin 101 (SR101), which labels astrocytes both in health and in peak EAE (8). These results are similar to the overlap measured in healthy $C X 3 C R 1^{+/-}$EGFP mice labeled by i.v. injection with SR101 $(2.7 \pm 1.1 \%$ overlap, Figure 1C).

\section{Intravital Imaging Reveals Morphologic Features of EAE Remission in the CNS}

We performed intravital imaging experiments in the brain stem of CerTN L15 $\times$ LysM tdRFP mice (neurons express the $\mathrm{Ca}^{2+}$ indicator TN L15, while predominantly $L y s M^{+}$phagocytes express tdRFP) and of CX3CR1 ${ }^{+/-}$EGFP mice (microglia/macrophages express EGFP, while predominantly astrocytes are labeled by SR101).

Infiltration of the CNS by Lys $\mathrm{M}^{+}$cells is transient, and varies with the stage of disease. In health, practically no Lys $M^{+}$cells are present except for few perivascular $L y s M^{+}$microglia. During peak of EAE, many Lys $M^{+}$cells are present within CNS lesions, and they mostly disappear during the remission phase. We could only identify isolated regions where $\mathrm{Lys}^{+}$cells were present inside or in the close proximity to blood vessels or meninges (Figure 2A).

In contrast to the peripheral immune cells, the inflammatoryinduced gliosis of CNS-resident cells [microglia and astrocytes having phagocytic capacity (8)] persists after EAE recovery (Figure 2B). We evaluated shape and function of astrocytes and microglia to test our hypothesis that the function of these CNS cells, in chronic neuroinflammation, has persistently (pathologically) elevated phagocytic features, even in the absence of peripheral immune cells.

First, we quantitatively analyzed the shape of microglia, based on the fact that resting microglia, typical for healthy CNS, are highly ramified, whereas activated microglia, especially those having a phagocytic function, lose their cellular processes and adopt an amoeboid shape. The amoeboid shapes are expected to be found especially in the diseased CNS (10).

We used Fourier coefficients to quantify and reproduce the ramified shape of microglia and to quantify their shape changes in the remission phase as compared to health and peak of the disease. Briefly, single microglia cells were segmented from intravital microscopy data acquired in the brain stem of healthy and EAE mice (in peak and remission phase). Six two-dimensional projections from each three-dimensional object (cell) were generated, and their shape was approximated by overlapping circles as displayed in Figure 2C. Each layer of circles is mathematically characterized by a scalar parameter called Fourier coefficient. Thus, the first Fourier coefficient defines the position of a cell, the second defines its dimensions by approximating it with a perfect 

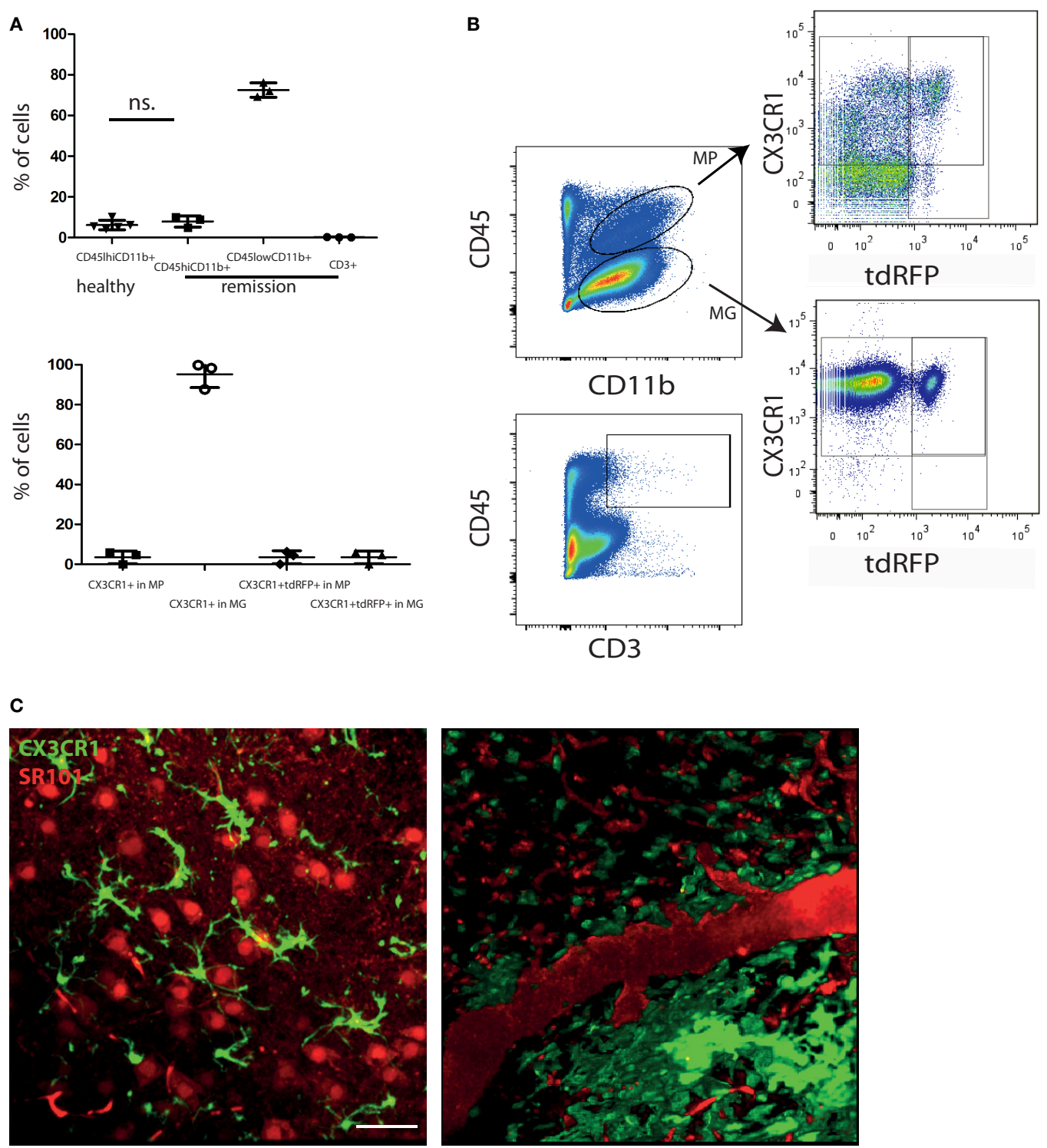

FIGURE 1 | Peripheral immune infiltration of the CNS has largely resolved in the remission phase of EAE. (A) FACS analysis of CNS cells after recovery of EAE shows a low immune infiltrate with few monocytes/macrophages (CD45 high CD11 b+; MP) and CD3 ${ }^{+}$cells. Most of the CD45 expressing cells are CD45 ${ }^{\text {low }} \mathrm{CD} 11 \mathrm{~b}^{+}$ (microglia; MG). CD45 high $\mathrm{CD} 11 \mathrm{~b}^{+}$frequencies are comparable to healthy untreated mice $(n=5 ; \pm \mathrm{SD})$. We applied an unpaired $t$-test to statistically evaluate the results. In the $C D 45^{\text {high }} \mathrm{CD} 11 \mathrm{~b}^{+}$fraction (MP), only few cells express CX3CR1 but most of the cells in the CD45 ${ }^{\text {low }} \mathrm{CD} 11 \mathrm{~b}^{+}$fraction (MG). The overlap of CX3CR1 and tdRFP was comparable in both cell types around $3 \%(n=3 ; \pm$ SD, clinical information listed in Table 1) (B) Exemplarily gating strategy of the FACS analysis of whole CNS in mice after recovery of EAE. (C) Projection of $3 \mathrm{D}$ intravital fluorescence image in the brain stem of a CX3CR1+/- EGFP mouse in health and during the remission phase of EAE. The astrocytes (and blood vessels) are labeled by i.v. injected SR101 (red), while the microglia are expressing EGFP (green). Scale bar $=50 \mu \mathrm{m}$. The colocalization of the EGFP and SR101 signals, i.e., overlap of the microglial and astrocytic markers, respectively, amounts to $3.6 \pm 1.8 \%$.

sphere, and the next Fourier coefficients define the number and length of cellular processes. Each cellular process is approximated by a set of spheres of various diameters, with the center on the surface of the most distant, previous sphere (Figure 2C). The higher the ramification and the length of cellular processes, the larger are the relative values of the high-order Fourier coefficients with respect to the second Fourier coefficient. We found a high shape similarity of microglia during the remission phase (118 cells) and of those imaged at the lesion site, at the peak of the disease (57 cells). In comparison to resting microglia in healthy controls (71 cells), the similarity was rather low (Figure 2D). The third, fourth, and fifth Fourier coefficients show a significant difference (using an ANOVA test) both in remission and in peak as compared to healthy controls. The results encompass two independent EAE experiments with $n=3$ healthy controls, $n=2$ mice at peak EAE, and $n=4$ mice during the remission phase. The findings of our intravital experiments demonstrate that in remission, after clinical recovery, microglia retain an 
TABLE 1 | Mouse strains, EAE data of the mice and mean NOX activation area values within lesions or gliosis/astrogliosis areas with SD per animal (6-20 areas within the brain stem per animal).

\begin{tabular}{|c|c|c|c|c|c|}
\hline EAE ID & Mouse strain & $\begin{array}{c}\text { EAE score at analysis } \\
\text { time point }\end{array}$ & Maximum EAE score & $\begin{array}{c}\text { Mean NOX activation } \\
\text { area (\%) }\end{array}$ & SD \\
\hline 1 & CX3CR1.EGFP & 1.5 & 1.5 & 17.88 & 5.13 \\
\hline 1 & CerTN L15 × LysM tdRFP & 1.0 & 1.0 & 13.20 & 1.06 \\
\hline 1 & CerTN L15 × LysM tdRFP & 0.5 & 2.0 & 8.15 & 2.25 \\
\hline 2 & CerTN L15 × LysM tdRFP & 2.5 & 2.5 & 16.99 & 9.02 \\
\hline 2 & CerTN L15 x LysM tdRFP & 0.5 & 1.5 & 9.91 & 3.97 \\
\hline 3 & CerTN L15 × LysM tdRFP & 2.0 & 2.0 & 10.65 & 0.51 \\
\hline 3 & CerTN L15 × LysM tdRFP & 2.0 & 2.0 & 7.18 & 1.42 \\
\hline 3 & CX3CR1.EGFP & 1.5 & 1.5 & 11.61 & 1.34 \\
\hline 3 & CX3CR1.EGFP & 0.0 & 1.5 & 9.27 & 3.25 \\
\hline 3 & CX3CR1.EGFP & 0.0 & 1.0 & 8.77 & 3.97 \\
\hline 4 & CX3CR1.EGFP & 0.0 & 2.0 & 11.53 & 5.38 \\
\hline 4 & CX3CR1.EGFP & 0.0 & 1.5 & 9.71 & 2.79 \\
\hline 4 & CX3CR1.EGFP & 0.5 & 2.0 & 12.19 & 4.65 \\
\hline 4 & CerTN L15 × LysM tdRFP & 0.0 & 2.0 & - & - \\
\hline 5 & CerTN L15 x LysM tdRFP & 0.5 & 3.5 & - & - \\
\hline 5 & CerTN L15 × LysM tdRFP & 0.5 & 3.5 & - & - \\
\hline $\begin{array}{l}\text { Healthy } \\
\text { controls }\end{array}$ & Mouse strain & & & $\begin{array}{c}\text { Mean NOX activation } \\
\text { area }(\%)\end{array}$ & SD \\
\hline 1 & CerTN L15 × LysM tdRFP & & & 0.37 & 0.13 \\
\hline 2 & $\mathrm{C} 57 \mathrm{BL} / 6$ & & & 2.84 & 0.29 \\
\hline 3 & CerTN L15 $\times$ LysM tdRFP & & & 0.47 & 0.09 \\
\hline 4 & C57BL/6 & & & 0.60 & 0.28 \\
\hline 5 & CerTN L15 × LysM tdRFP & & & 2.08 & 0.91 \\
\hline
\end{tabular}

We included five independent EAE experiments and five healthy controls for the intravital NAD(P)H-FLIM experiments.

activated morphology, suggesting that their function remains predominantly phagocytic despite the fact that clinical symptoms disappeared.

Consistent with the results of shape analysis of microglia, the astrocytic network appears intact in healthy controls $(n=2)$, whereas during peak $\operatorname{EAE}(n=4)$ and the remission phase $(n=3)$, it appears disrupted (Figure 2E). Additionally, the fine astrocytic processes completely disappear and are replaced by thick perivascular processes, while the astrocytic cell bodies adopt ameboid shapes (Figure 2E). A quantification of these observations is rather difficult. Even if a good segmentation of the single astrocytes and their processes is given, currently there is no available mathematical approach or set of mathematical parameters to summarize the complexity of the profound changes of the astrocytic network. However, altogether the observations regarding morphological modifications suggest that the astrocytes are also shifted toward a phagocytic function.

\section{Subclinical Neuronal Dysfunction Correlates with Oxidative Stress without Overt Immune Infiltration after Recovery of EAE}

Altered morphology often indicates functional changes, but morphology is not a direct measure of the cellular function. To evaluate alterations in cellular function over the course of EAE, we used intravital $\mathrm{NAD}(\mathrm{P}) \mathrm{H}$ fluorescence lifetime imaging (FLIM), as previously described (8), to detect the over-activation of NADPH oxidases (NOX1-4, DUOX1, 2). As we previously showed in intravital imaging experiments of mice affected by EAE, a concentration of $\sim 200 \mu \mathrm{M}$ of ROS is detectable in the brain stem, in EAE, using local ROS labeling with Amplex Red. In contrast, in healthy animals, we could not detect any ROS generation. As ROS molecules are highly reactive and diffusive, their detection is limited and the analysis of their cellular source practically impossible. We circumvent this disadvantage by detecting the catalyzer of ROS production, i.e., NOX enzymes, using NAD(P)H-FLIM in vivo. We previously showed that high ROS concentration in the brain stem of EAE animals correlates with the over-activation of NOX enzymes as detected by intravital NAD(P)H-FLIM (12). The fluorescence lifetime of NADPH bound to NADPH oxidases is $\sim 3650$ ps (12), differing from generally active NADH- and $\mathrm{NADPH}$-dependent enzymes [fluorescence lifetime of $\mathrm{NAD}(\mathrm{P}) \mathrm{H}$ $\sim 2200$ ps]. The over-activation of NADPH oxidases is a prerequisite of oxidative stress - known to be one of the main causes of neuronal dysfunction in chronic neuroinflammation $(6,13)$.

In healthy mice, intravital $\mathrm{NAD}(\mathrm{P}) \mathrm{H}-\mathrm{FLIM}$ of the brain stem reveals predominantly metabolic enzyme activity (8). At peak of EAE, the lesion site is associated with vast areas of activated NADPH oxidases, leading to increased oxidative stress (8). Surprisingly, even if overt inflammation and the clinical symptoms disappear, a local activation of NADPH oxidases does not decline to levels found in healthy mice. While the area of NOX enzymes activation in the brain stem of healthy mice amounts in average to $1.8 \pm 1.3 \%$, the same average value at peak of the disease significantly increases eightfold to $15.6 \pm 5.1 \%$ and declines only slightly to $9.4 \pm 1 \%$ during the remission phase, still over fivefold higher than in healthy mice (Figures 3A,C). 

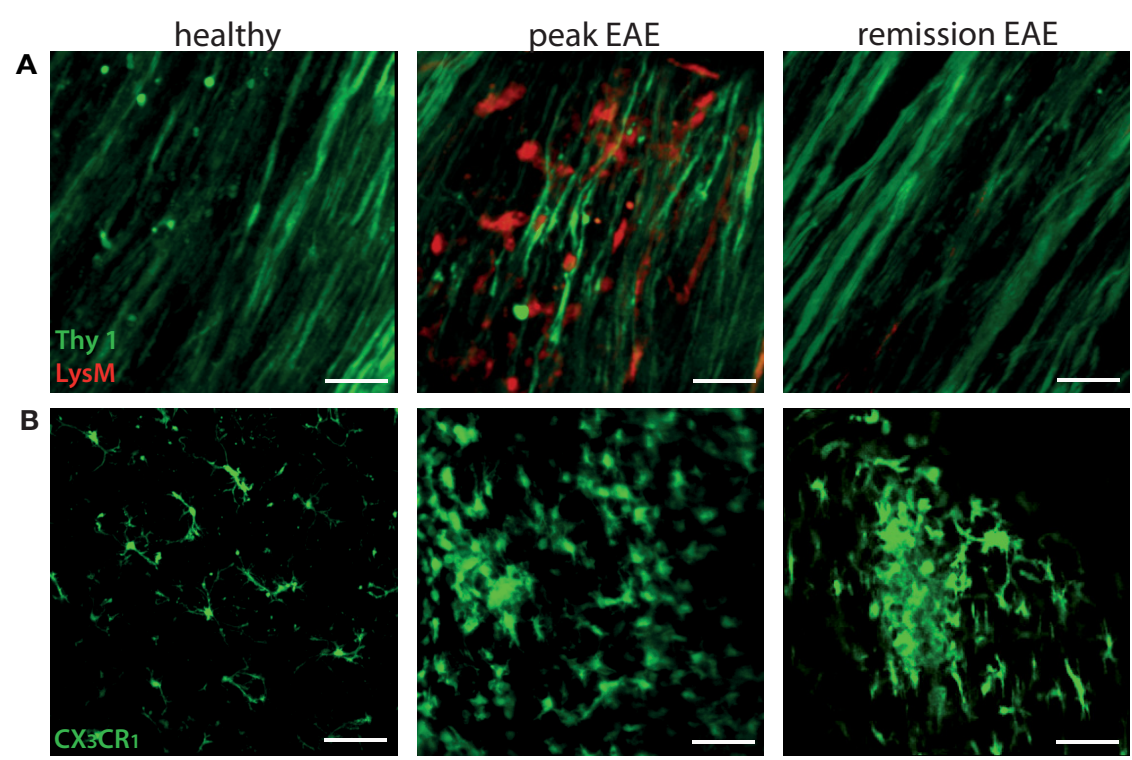

C
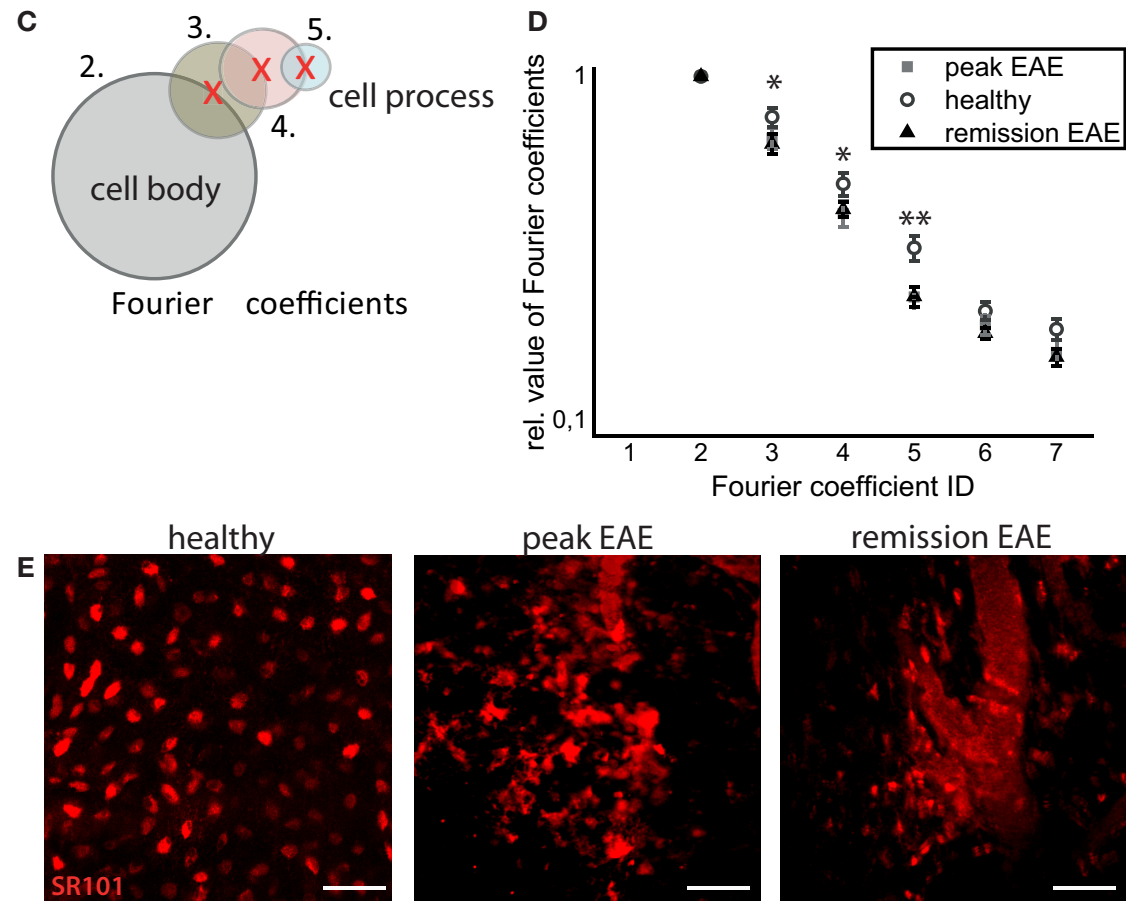

remission $\mathrm{EAE}$

FIGURE 2 | Intravital imaging reveals that remission in EAE correlates with lack of overt immune infiltration, with persisting disruptions of the microglial and astrocytic networks. (A) 3D intravital fluorescence images in the brain stem of CerTN L15 $\times$ LysM tdRFP mice in health ( $n=5$ ), at peak EAE $(n=6)$ and in the remission phase $(n=2) . \lambda_{\text {exc }}=850+1110 \mathrm{~nm}, \lambda_{\text {em }}=525 \pm 25 \mathrm{~nm}$ (Thy1-Citrine in neurons depicted in green), $\lambda_{\text {em }}=593 \pm 20 \mathrm{~nm}$ (LysM tdRFP in phagocytes depicted in red), scale bar $=50 \mu \mathrm{m}$. (B) 3D intravital fluorescence images in the brain stem of $C X 3 C R 1^{+/-} E G F P$ mice in health $(n=3)$, at peak EAE $(n=4)$ and in the remission phase $(n=5)$. $\lambda_{e x c}=935 \mathrm{~nm}, \lambda_{\mathrm{em}}=525 \pm 25 \mathrm{~nm}\left(\mathrm{CX} 3 \mathrm{CR} 1^{+/-}\right.$EGFP in microglia/macrophages depicted in green), scale bar $=50 \mu \mathrm{m}$. (C) Using higher-order Fourier coefficients, we describe the complex shape of microglia. The first Fourier coefficient describes the position of the cells, the second coefficient the sphericity of the cell body and starting from the third Fourier coefficient, the ramification of all cell processes is reproduced: the higher the values of high-order Fourier coefficients with respect to the second Fourier coefficient, the higher the degree of ramification and length of cellular processes of microglia.

(D) The different shapes of the microglia, shown in (B), were classified in health (71 cells) at peak EAE (57 cells) and in its remission phase (63 cells). The difference between the values of the third, fourth, and fifth Fourier coefficients is significant between healthy controls and remission, but not significant between peak of EAE and remission of EAE. Statistical significance was determined by ANOVA ( $\left.{ }^{*} p<0.05,{ }^{* *} p<0.01,{ }^{* * *} p<0.001\right)$. (E) Projection of 3D intravital fluorescence images in the brain stem of C57/B6 mice i.v. injected with sulforhodamine 101 (SR101) in health $(n=2)$, at peak EAE $(n=4)$ and in the remission phase $(n=3)$. $\lambda_{\text {exc }}=880$ nm, $\lambda_{\text {em }}=593 \pm 20 \mathrm{~nm}$ (SR101 in astrocytes depicted in red), scale bar $=50 \mu \mathrm{m}$. 
A

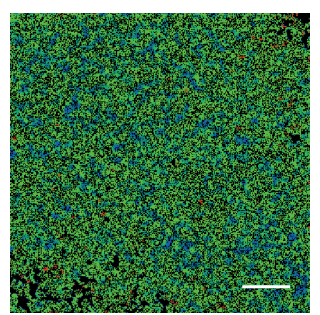

healthy

B
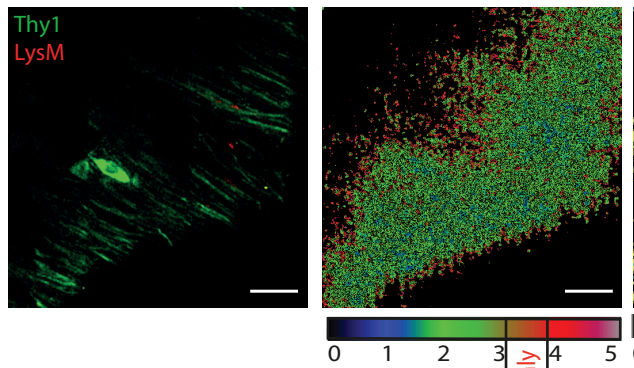

$\tau_{2} / \mathrm{ns}$

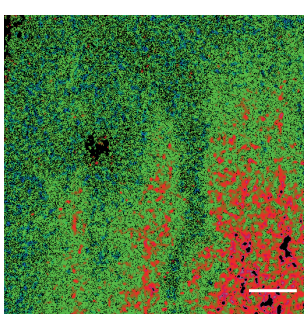

peak EAE: lesion site

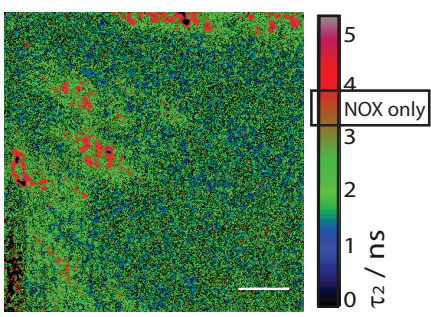

remission $\mathrm{EAE}$

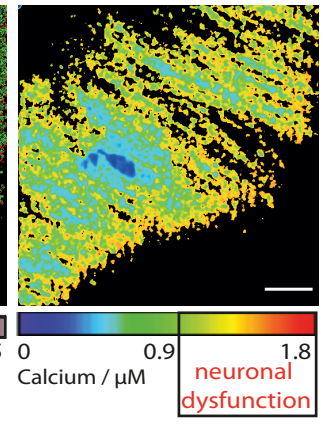

C
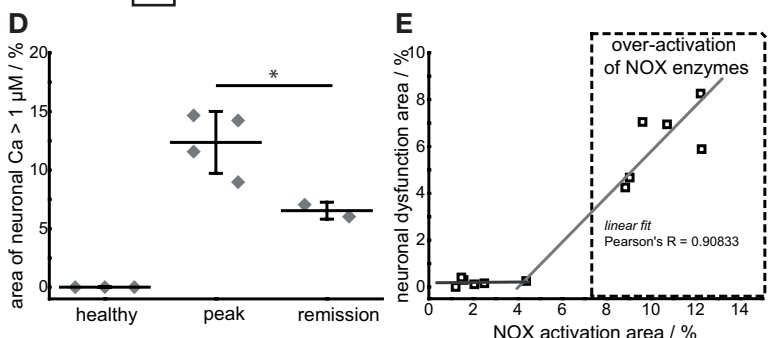

F
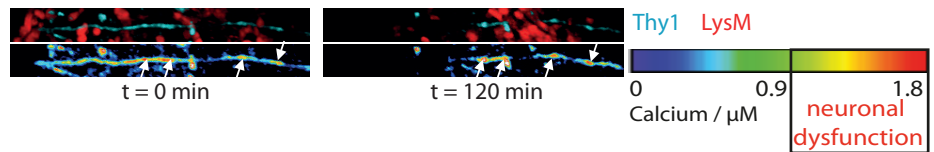

FIGURE 3 | NOX enzymes activation correlates with neuronal dysfunction in the remission phase of EAE. (A) $\tau_{2}($ enzyme-bound) NAD(P)H-FLIM images acquired in the brain stem of healthy mice $(n=5)$ and of mice affected by EAE at peak of the disease $(n=6)$ and in the remission phase $(n=7)$. The results encompass four independent EAE experiments. Scale bar $=30 \mu \mathrm{m}$. (B) Intravital fluorescence intensity image, NAD(P)H-FLIM image and FRET-FLIM neuronal calcium image acquired in the brain stem of a CerTN L15 $\times$ LysM tdRFP mouse in the remission phase of EAE. Scale bar $=30 \mu \mathrm{m}$. (C) Quantification of the mean NOX activation area of individual mice at peak EAE $(n=6)$ and in the remission phase $(n=7)$, four independent EAE experiments. While the mean are of NOX activation is strongly increased in the remission phase of EAE as compared to healthy controls, we could observe only a slight decrease of the NOX activation area as compared to peak of EAE (at lesion sites). (D) Quantification of the neuronal dysfunction area characterized by a neuronal calcium concentration larger than $1 \mu \mathrm{M}$ at peak $\operatorname{EAE}(n=4)$ and in the remission phase $(n=2)$, two independent EAE experiments. The area of elevated neuronal calcium (area of neuronal dysfunction) is slightly reduced in the remission phase of EAE as compared to the peak of the disease. However, since in healthy controls there is no elevated neuronal calcium, in both phases of EAE, the elevated calcium indicates massive neuronal dysfunction. (E) Direct correlation between NOX enzymes over-activation area and neuronal dysfunction area in the remission phase of EAE, within the brain stem ( $n=2$ mice). All images are acquired at 30-150 $\mu \mathrm{m}$ depth within the brain stem ( $z$ -

step $=2 \mu \mathrm{m})$. (F) Intravital 3D images of the brain stem of a CerTN L15 $\times$ tdRFP mouse affected by EAE, at peak of the disease, at an arbitrary time point $t=0$ and 120 min later. The upper panels show intensity images of axons (cyan, Thy 1 ) and of immune cells (red), whereas the lower images show the corresponding FRET ratio images (Calcium images) of the axons. At the contact sites between axons and immune cells, we observe strongly increased neuronal calcium. After $2 \mathrm{~h}$, at exactly these sites, we observed dramatic morphological changes of the axons, i.e., appearance of ovoid bodies and axonal disruption. The axonal disruption and ovoid bodies formation along the axon is indicated by white arrows in the lower panels of (F). Statistical evaluation in (C,D) was determined by ANOVA tests $\left({ }^{*} p<0.05,{ }^{* *} p<0.01,{ }^{* *} p<0.001\right)$.

To determine if this increased activation of NAD $(\mathrm{P}) \mathrm{H}$ oxidases was associated with subclinical neuronal dysfunction, we determined the neuronal calcium level using intravital FRET-FLIM in the brain stem of CerTN L $15 \times$ LysM tdRFP mice affected by EAE, as previously described $(8,14,15)$. Associated with the persisting oxidative stress, we observed increased neuronal calcium indicating subclinical neuronal dysfunction, and progressing to neuronal damage, within the areas of elevated oxidative stress. The area of measured neuronal dysfunction in the remission phase $(6.2 \pm 1.7 \%)$ is lower than at peak of disease $(11.7 \pm 2.8 \%)(16)$, 
A

fluorescence intensity
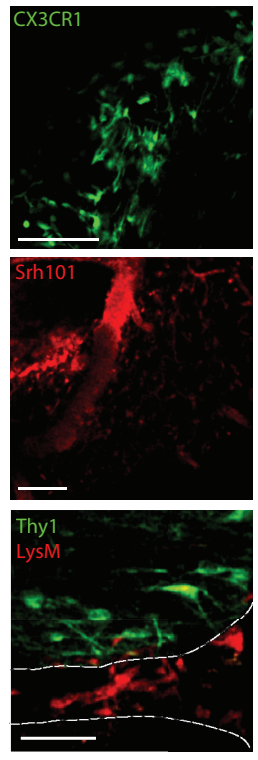

$\tau_{2} \mathrm{NAD}(\mathrm{P}) \mathrm{H}$ lifetime images

\section{all cells in tissue cell subset specific}
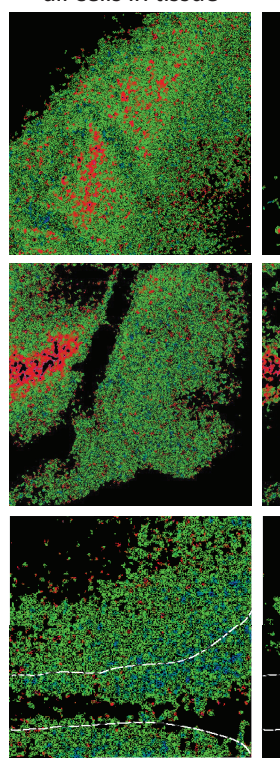

0
B
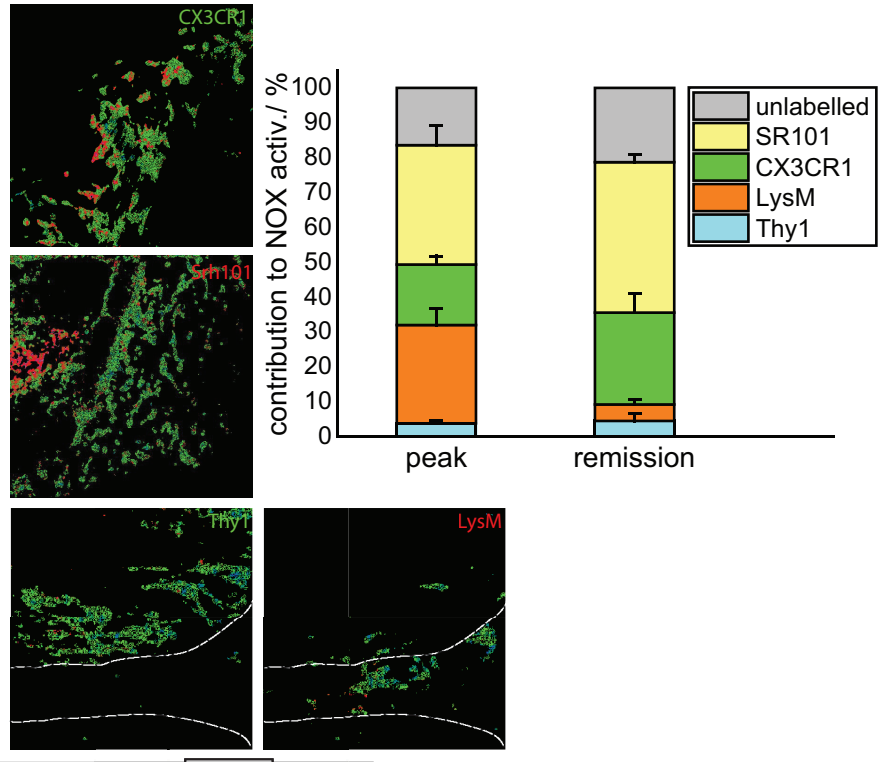

$\tau_{2} / \mathrm{ns}$

FIGURE 4 | Cellular origin of oxidative stress in the remission phase of EAE. (A) Intravital fluorescence images and enzyme-bound NAD(P)H fluorescence lifetime images of all cells and of specific cell subtypes acquired in the brain stem of transgenic mice affected by EAE, in the remission phase. We evaluated CerTN L15 $\times$ LysM tdRFP mice, in which neurons Thy1 express the calcium indicator TN L15 and LysM+ phagocytes express tdRFP, and CX3CR1+/- EGFP mice, in which microglia/macrophages express EGFP. Astrocytes were intravitally labeled with sulforhodamine 101 by i.v. injection at least $2 \mathrm{~h}$ before imaging. The white lines in the fluorescence and fluorescence lifetime images at the bottom of (A) demarcate the outline of a blood vessel. Hence, it becomes obvious that most of $L y s M^{+}$cells reside within the blood vessels, and only few can be found at the border to the CNS parenchyma. Scale bars $=50 \mu \mathrm{m}$. (B) Contribution of the individual cell subsets to the over-activation of the NOX enzymes in the CNS of mice during the remission phase of EAE ( $n=2$ CerTN L15 $\times$ LysM tdRFP mice - neurons/Thy 1 and LysM; $n=5$ CX3CR1 ${ }^{+/}$EGFP mice - microglia/CX3CR1 cells; $n=3$ mice labeled with sulforhodamine 101 - astrocytes). The results encompass four independent EAE experiments.

in line with higher scores during peak than in the recovery phase (Table 1); however, it is significantly higher than in healthy mice, in which no neuronal dysfunction can be observed using the same approach (Figures 3B,D) (14). As depicted in Figure 3E, in regions with no or very low over-activation of NOX enzymes ( $<4 \%$ of the total observed area), no neuronal dysfunction can be detected. Beyond this value, the oxidative stress regime is established and neuronal dysfunction linearly increases with increasing area of NOX enzymes activation, within the CNS (Pearson's $R=0.90883$ ).

As we and others previously demonstrated, a sustained elevated calcium concentration in neurons, both in vivo and in primary neuronal cultures, can precede morphological changes and finally neuronal death. In the brain stem of a CerTN L15 $\times$ LysM tdRFP mouse affected by EAE, enhanced contact of axons with peripheral LysM cells correlate with an increased calcium baseline in axons (Figure 3F). Imaging over $2 \mathrm{~h}$ reveals after recovery of EAE sites of increased calcium concentration do not correlate with dramatic morphological changes such as ovoid bodies or even axonal disruption, in contrast to the situation in peak EAE as depicted in Figure 3F (white arrows). Since the TN L15 genetically encoded biosensor reacts slowly to calcium (within few hundred milliseconds), it cannot track the physiologic calcium oscillations typical for neurons, but records only the low average baseline ( $\approx 100 \mathrm{nM})(14)$.

\section{Microglia and Astrocytes Mainly Contribute to Oxidative Stress after EAE Recovery}

Next, we elucidated the specific cellular origin of the persistent oxidative stress in the CNS during the remission phase. The approach used in our study - performing endogenous $\mathrm{NAD}(\mathrm{P}) \mathrm{H}-\mathrm{FLIM}$ in the CNS of mice with differently fluorescing cell subsets $\left(L y s M^{+} t d R F P, C e r T N L 15, C X 3 C R 1^{+/-}\right.$EGFP, or SR101 labeled cells) affected by EAE - enables the direct identification of specific cellular origins of oxidative stress by examining colocalization of assembled NOX enzymes acquired via NAD(P) $\mathrm{H}-\mathrm{FLIM}$ with cellular markers visualized by fluorescence imaging (Figures 4A,B).

We quantified the contribution of specific cell types to the total area of NOX enzymes activation in the CNS and found that the mean contribution of $\mathrm{LysM}^{+}$phagocytes amounts to maximally $4.3 \%$, a value comparable to that of neurons (Thy $1^{+}$cells, $4.7 \%$ ). 
Whereas the contribution of neurons to the area of oxidative stress generation is, as expected, similar at peak EAE and during the remission phase, $L y s M^{+}$phagocytes proved to be a major source of massive ROS production only during peak EAE but not during the remission, due to their low frequency within the CNS in this phase. Only few perivascular macrophages could be detected (Figure 4A, bottom panel) in line with the results of our FACS analysis in Figure 1A (5\% of the cells are tdRFP expressing) and complementary to Figure 2A, in which no LysM tdRFP cell infiltration is shown after EAE recovery. LysM tdRFP cells can are located only in closest proximity to or within blood vessels (marked by white lines in Figure 4A) and are, even then, only partially activated (Figure 4A, bottom panel right image).

The main contribution to ROS production after recovery in EAE is associated with $C X 3 C R 1^{+}$cells, i.e., $26 \%$, and astrocytes (SR101), i.e., 45\% (Figure 4B). Thus, microglia and astrocytes together contribute over $70 \%$ of the oxidative stress generation in remission of EAE. Since only in half of the microglia EGFP is detectable by intravital microscopy (heterozygous breeding) and not the entire astrocyte population takes up SR101 [i.e., only half of $\mathrm{GFAP}^{+}$cells, namely GFAP low (8)], the frequencies of microglia and astrocytes may well be underestimated, and we expect an even higher effective contribution of these cell types to oxidative stress.

\section{DISCUSSION}

It is widely accepted that during chronic neuroinflammation, both in the human disease MS and in its murine model EAE, oxidative stress plays a major role in demyelination and neuronal damage (6-8). Most of the findings reported in EAE studies resulted from investigations during the peak of inflammation (17). After peak of disease, in C57BL/6 mice immunized with $\mathrm{MOG}_{35-55}$ peptide (7), clinical signs can resolve spontaneously $(10,17)$ or the mice enter a chronic phase with persistent paralysis (1). This pattern resembles features of the human disease MS, since in its relapsing-remitting phase (RRMS) symptoms are normally self-limiting and patients spontaneously recover.

Despite our knowledge about the dynamic processes of the acute immune response in the CNS, information about the reaction of the tissue after the inflammatory attack is very limited. The processes determining how and where new lesions occur are difficult to study in human tissue as the majority of the samples derives either from very early (biopsies) or very late (post-mortem) lesions. How lesions resolve, how progression takes place, and other characteristics of the later phases of the disease are not well understood $(3,18-20)$. The alterations of the immune system in MS lesions are obvious, but how this impacts the function of the CNS tissue is almost unknown. Only few inflammatory animal models focus on remyelination as a tissue response, e.g., studies using models of inflammatory cortical demyelination $(18,19)$.

In this study, we addressed the question of tissue damage regarding oxidative stress as a major detrimental factor for the cells within CNS tissue and consecutive ongoing subclinical neuronal dysfunction after clinical remission of EAE signs. We observed that astrocytes and microglia are shifted toward an activated phenotype, showing both changes in morphology and, even more striking, a high level of activated NADPH oxidases correlating with persisting neuronal elevated calcium levels without evident morphological alterations. Hence, the consequences of sustained neuronal calcium levels after EAE recovery in contrast to peak of EAE are not clear yet (16). Could this be a reason for long-term neuronal damage leading to a progressive disease course in humans?

The role and fate of microglia/macrophages during neuroinflammation is still not well understood (10, 20). From BrdU studies, it was concluded that although microglia enter the cell cycle during acute inflammation, they return to quiescence following remission (21). Furthermore, gene expression analysis of microglia and macrophages suggested that after EAE recovery, these cells return toward homeostasis (10). In contrast, our data demonstrate that at least a subpopulation of microglia is not quiescent and still retains their activated function during remission, as demonstrated by their ameboid morphology and enhanced NOX activity in our model.

The results of our present study indicate that even after remission of the clinical signs, astroglial scars represent areas of ongoing tissue damage, even in the absence of peripheral immune cells. Our findings support the idea of a "trapped" ongoing CNS inflammation as a mechanism of chronic progression in MS (2). At this phase of the disease, astrocytes and microglia alone are a source of persistent oxidative stress locally correlating with ongoing subclinical neuronal dysfunction, as measured by pathologically increased calcium levels in CerTN L15 $\times$ LysM $t d R F P$ mice.

Our model provides a useful tool to further investigate the "tissue memory" of neuroinflammatory processes, in order to better understand mechanisms of chronicity and disease progression in MS.

\section{MATERIALS AND METHODS}

\section{Two-Photon Laser-Scanning Microscopy}

Both fluorescence intensity and FLIM experiments were performed using a specialized two-photon laser-scanning microscope based on a commercial scan head (TriMScope, LaVision BioTec, Bielefeld, Germany). The detection of the fluorescence signals was accomplished either with photomultiplier tubes in the ranges $460 \pm 30,525 \pm 25,593 \pm 20 \mathrm{~nm}$ or with a 16-channel parallelized TCSPC detector (FLIM-X $\mathrm{X}_{16}$, LaVision BioTec, Bielefeld, Germany) in the range $460 \pm 30 \mathrm{~nm}$. The excitation of NADH and NADPH was performed at $760 \mathrm{~nm}$ (detection at $460 \pm 30 \mathrm{~nm}$ ), of Cerulean (detection at $460 \pm 30 \mathrm{~nm}$ ), SR101 (detection at $593 \pm 20 \mathrm{~nm}$ ), and EGFP (detection at $525 \pm 25 \mathrm{~nm}$ ) at 850 or $880 \mathrm{~nm}$, and of tdRFP at $1110 \mathrm{~nm}$ (detection at $593 \pm 20 \mathrm{~nm}$ ).

For both intensity and fluorescence lifetime imaging, we used an average maximum laser power of $8 \mathrm{~mW}$ to avoid photodamage. The experimental parameters for FLIM were 160 ps histogram bin [for NAD(P)H-FLIM] and 80 ps histogram bin (for FRETFLIM) and maximum acquisition time for a $512 \times 512$ image was $5 \mathrm{~s}$ to record a fluorescence decay stack. The time-window in which the fluorescence decays were acquired was set to 9 ns. 


\section{Data Analysis}

Three-dimensional intravital images acquired within the brain stem of $C X 3 C R 1^{+-}$EGFP mice, either healthy or affected by EAE, at peak and in the remission phase of the disease, were segmented using image analysis software (Imaris, BitPlane, UK). The 3D surfaces of the segmented cells (50-200 cells per condition) were transferred to Fiji/ImageJ and six orthogonal projections were generated for each cell. For each projection of each cell, the Fourier coefficients, describing the sphericity vs. ramification of the cell, were calculated (custom plug-in available in Fiji developed by Zoltan Cseresnyes). All Fourier coefficients corresponding to a single cell were merged following a linear algebraic combination to describe all ramifications of the cell (cell processes). The rank of the Fourier coefficients describes increasingly complex cellular ramifications: whereas the first Fourier coefficient corresponds to the first spherical approximation of the cell, the next coefficients describe increasingly longer processes.

Fluorescence lifetime imaging data analysis was performed using self-written software, as previously described $(12,14)$. The time-resolved fluorescence signal of $\mathrm{NAD}(\mathrm{P}) \mathrm{H}$, as acquired by the TCSPC device, was approximated with a bi-exponential decay function (Eq. 1):

$$
I_{\mathrm{NAD}(\mathrm{P}) \mathrm{H}}(t)=\varepsilon+a_{1} \times e^{-\mathrm{t} / \tau_{1}}+a_{2} \times e^{-\mathrm{t} / \tau_{2}}
$$

with $\varepsilon$ the background, the 1 -indexed term of the sum representing the fluorescence decay of free NADH and NADPH and the 2-indexed term representing the fluorescence decay of enzyme-bound $\mathrm{NADH}$ and $\mathrm{NADPH}$. The fluorescence lifetime $\tau_{1}$ [free $\mathrm{NAD}(\mathrm{P}) \mathrm{H}$ ] is $400-450 \mathrm{ps}$, while the fluorescence lifetime $\tau_{2}$ of $\mathrm{NAD}(\mathrm{P}) \mathrm{H}$ bound to metabolic enzymes has an average of $\sim 2000$ ps. If bound to NADPH oxidases (NOX1-4, DUOX1, 2), NADPH shows a typical fluorescence lifetime of 3650 ps, independent of cell type and even of species, since we repeatedly measured this value in various cell types of humans, mice, and even plants (Nicotiana tabacum). We focused all through the manuscript on the fluorescence lifetime $\tau_{2}$ of the enzyme bound NAD $(\mathrm{P}) \mathrm{H}$.

As previously described, we define the area of neuronal dysfunction as the area of free neuronal calcium exceeding a concentration of $1 \mu \mathrm{M}$ (14). The neuronal calcium concentration was measured in vivo using FLIM, in mice expressing the FRET-based calcium biosensor TN L15 in Thy $1^{+}$cells. Thereby, the time-resolved fluorescence decay of the donor in the FRET construct (Cerulean) was also approximated by a biexponential function (Eq. 2):

$$
I_{\text {Cerulean }}(t)=\varepsilon+a_{1} \times e^{-\mathrm{t} / \tau_{1}}+a_{2} \times e^{-\mathrm{t} / \tau_{2}}
$$

with $\varepsilon$ the background, the 1 -indexed term representing the fluorescence decay of the FRET-quenched donor and the 2-indexed term representing the fluorescence decay of the unquenched donor. Here, we focused on the ratio $a_{1} /\left(a_{1}+a_{2}\right)$ of the relative concentrations of the FRET-quenched $a_{1}$ and unquenched Cerulean $a_{2}$, and, using our previously published calibration curve, we determined the absolute calcium concentration within neurons.
Statistical analysis and graphical presentation was carried out with GraphPad Prism 4 (Graphpad Software, USA) and OriginPro (OriginLab, USA). Results are shown as mean values from analyzed data per mouse, in addition the mean \pm SD summarize collective data from performed experiments.

\section{Mice}

All mice used were on a C57BL/6 background. The CerTN L15 $\times$ LysM tdRFP mouse expresses a FRET-based calcium biosensor consisting of Cerulean (donor) and Citrine (acceptor) bound to troponin $\mathrm{C}$, a calcium-sensitive protein present in certain subsets of neurons (22). Additionally, tdRFP is expressed in LysM $^{+}$cells. The CX3CR1 $1^{+-}$EGFP mouse was used to detect microglia (Table 1).

\section{EAE Induction}

Experimental autoimmune encephalomyelitis was induced as previously described. Briefly, mice were immunized subcutaneously with $150 \mu \mathrm{g}$ of $\mathrm{MOG}_{35-55}$ (Pepceuticals, UK) emulsified in CFA (BD Difco, Germany) and received $200 \mathrm{ng}$ pertussis toxin (PTx, List Biological Laboratories, Inc.) intraperitoneally at the time of immunization and $48 \mathrm{~h}$ later. Intravital multi-photon microscopy was performed at different stages of the disease, i.e., peak (3-7 days after appearance of first clinical symptoms) and remission (after decline of clinical symptoms to a score $\leq 0.5$ ). Mice were randomly picked for analysis. Detailed information about the performed EAE runs and individual scores of the mice are listed in Table 1. Mice at peak of disease were part of a previous study of Mossakowski et al. (8) and serve as a reference in this study. We did not included animals that never got sick and at onset analyzed animals.

\section{Preparation of the Brain Stem Window for Intravital Imaging}

As previously described, the brain stem was exposed by carefully removing the musculature above the dorsal neck area and removing the dura mater between the first cervical vertebra and the occipital skull bone. The head was inclined for access to deeper brainstem regions and the brain stem superfused with isotonic Ringer solution. Anesthesia depth was controlled by continuous $\mathrm{CO}_{2}$ measurements of exhaled gas and recorded with a CI-240 Microcapnograph (Columbus Instruments, USA) and by an Einthoven three-lead electrocardiogram (ECG). In order to avoid strong breathing artifacts in the brainstem of anesthetized mice, the ECG signal was correlated to the respiration rate and used as an external trigger for the image acquisition software, which controls the hardware of the microscope setup. Thus, each fluorescence stack was recorded at the same respiration state of the mouse and also in the same tissue region. Animal experiments were approved by the appropriate state committees for animal welfare (G0081/10, LAGeSo - Landesamt für Gesundheit und Soziales) and were performed in accordance with current guidelines and regulations.

\section{FACS Analysis}

To isolate cells from the whole brain and spinal cord of $L y s M$ tdRFP mice, the tissue was homogenized after PBS perfusion, and a percoll gradient was performed according to standard 
protocols with 25 and 75\% stock istotonic percoll (GE Helthcare) and HBSS. Cells were blocked with antibodies to Fc $\gamma$ receptors (DRFZ, clone 2.4G2) to avoid non-specific staining, and were subsequently stained with FITC-labeled PerCP-labled rat antiCD45 (BioLegend) or Cy5- (DRFZ), APC- or Pacific Blue ${ }^{\mathrm{TM}}$ (BioLegend)-labeled rat anti-CD11b, in some experiments fixable Viability Dye eFluor ${ }^{\circledR} 780$ (eBioscience), anti-CX3CR1 APC and anti-CD3 Brilliant Violet ${ }^{\mathrm{TM}}$ (both BioLegend) were used according to standard procedures, followed by fixation using $4 \%$ Paraformaldehyde (Electron Microscopy Science) for $10 \mathrm{~min}$. FACS analysis was performed on a LSR Fortessa (BD).

\section{AUTHOR CONTRIBUTIONS}

$\mathrm{DB}, \mathrm{HR}, \mathrm{RN}$, and RG designed and performed research and analyzed data. DB, HR, RN, and RL wrote the manuscript. HR and $\mathrm{RN}$ initiated, organized, and supervised the project. HR and $\mathrm{AH}$ provided expertise in mouse handling and intravital imaging

\section{REFERENCES}

1. Wekerle H. Lessons from multiple sclerosis: models, concepts, observations. Ann Rheum Dis (2008) 67(Suppl 3):iii56-60. doi:10.1136/ ard.2008.098020

2. Bradl M, Lassmann H. Progressive multiple sclerosis. Semin Immunopathol (2009) 31:455-65. doi:10.1007/s00281-009-0182-3

3. Stadelmann C, Wegner C, Bruck W. Inflammation, demyelination, and degeneration - recent insights from MS pathology. Biochim Biophys Acta (2011) 1812:275-82. doi:10.1016/j.bbadis.2010.07.007

4. Frischer JM, Bramow S, Dal-Bianco A, Lucchinetti CF, Rauschka H, Schmidbauer $\mathrm{M}$, et al. The relation between inflammation and neurodegeneration in multiple sclerosis brains. Brain (2009) 132:1175-89. doi:10.1093/ brain/awp070

5. Frohman EM, Racke MK, Raine CS. Multiple sclerosis - the plaque and its pathogenesis. N Eng J Med (2006) 354:942-55. doi:10.1056/ NEJMra052130

6. Fischer MT, Sharma R, Lim JL, Haider L, Frischer JM, Drexhage J, et al. NADPH oxidase expression in active multiple sclerosis lesions in relation to oxidative tissue damage and mitochondrial injury. Brain (2012) 135:886-99. doi:10.1093/brain/aws012

7. Nikić I, Merkler D, Sorbara C, Brinkoetter M, Kreutzfeldt M, Bareyre FM, et al. A reversible form of axon damage in experimental autoimmune encephalomyelitis and multiple sclerosis. Nat Med (2011) 17:495-9. doi:10.1038/ nm. 2324

8. Mossakowski AA, Pohlan J, Bremer D, Lindquist R, Millward JM, Bock M, et al. Tracking CNS and systemic sources of oxidative stress during the course of chronic neuroinflammation. Acta Neuropathol (2015) 130(6):799-814. doi:10.1007/s00401-015-1497-x

9. Croxford AL, Lanzinger M, Hartmann FJ, Schreiner B, Mair F, Pelczar P, et al. The cytokine GM-CSF drives the inflammatory signature of CCR2+ monocytes and licenses autoimmunity. Immunity (2015) 43:502-14. doi:10.1016/j. immuni.2015.08.010

10. Yamasaki R, Lu H, Butovsky O, Ohno N, Rietsch AM, Cialic R, et al. Differential roles of microglia and monocytes in the inflamed central nervous system. J Exp Med (2014) 211:1533-49. doi:10.1084/jem.20132477

11. Siffrin V, Brandt AU, Radbruch H, Herz J, Boldakowa N, Leuenberger T, et al. Differential immune cell dynamics in the CNS cause CD4+ T cell compartmentalization. Brain (2009) 132:1247-58. doi:10.1093/brain/awn354

12. Niesner R, Narang P, Spiecker H, Andresen V, Gericke KH, Gunzer M. Selective detection of NADPH oxidase in polymorphonuclear cells by means of NAD(P)H-based fluorescence lifetime imaging. J Biophys (2008) 2008:602639. doi:10.1155/2008/602639

13. Schuh C, Wimmer I, Hametner S, Haider L, Van Dam AM, Liblau RS, et al. Oxidative tissue injury in multiple sclerosis is only partly reflected and performed EAE experiments (G0081/10). ZC developed algorithms and analyzed data.

\section{ACKNOWLEDGMENTS}

We thank O. Griesbeck for providing the CerTN L15 transgenic mice and $\mathrm{H}$. J. Fehling for the Rosa26.tdRFP transgenic mice. We kindly thank R. Günther and P. Mex for excellent technical assistance. We are highly grateful to Frank Heppner who provided insight and expertise that greatly assisted the research.

\section{FUNDING}

We acknowledge the Deutsche Forschungsgemeinschaft under grant NI 1167/3-1, NI 1167/4-1 to RN as well as under the TRR130 TP17 and C01 to RN, HR, and AH for financial support. This work was partially funded through the excellence cluster NeuroCure (DFG EXC257) to AH.

in experimental disease models. Acta Neuropathol (2014) 128:247-66. doi:10.1007/s00401-014-1263-5

14. Rinnenthal JL, Börnchen C, Radbruch H, Andresen V, Mossakowski A, Siffrin V, et al. Parallelized TCSPC for dynamic intravital fluorescence lifetime imaging: quantifying neuronal dysfunction in neuroinflammation. PLoS One (2013) 8:e60100. doi:10.1371/journal.pone.0060100

15. Radbruch H, Bremer D, Mothes R, Günther R, Rinnenthal JL, Pohlan J, et al. Intravital FRET: probing cellular and tissue function in vivo. Int J Mol Sci (2015) 16:11713-27. doi:10.3390/ijms160511713

16. Siffrin V, Radbruch H, Glumm R, Niesner R, Paterka M, Herz J, et al. In vivo imaging of partially reversible th17 cell-induced neuronal dysfunction in the course of encephalomyelitis. Immunity (2010) 33:424-36. doi:10.1016/j. immuni.2010.08.018

17. Moreno B, Jukes JP, Vergara-Irigaray N, Errea O, Villoslada P, Perry VH, et al. Systemic inflammation induces axon injury during brain inflammation. Ann Neurol (2011) 70:932-42. doi:10.1002/ana.22550

18. Denis NL, Wrzos C, Brück W, Stadelmann C, Nessler S. A new mouse model of inflammatory cortical demyelination. J Neuroimmunol (2014) 275(1):119. doi:10.1016/j.jneuroim.2014.08.316

19. Merkler D, Ernsting T, Kerschensteiner M, Bruck W, Stadelmann C. A new focal EAE model of cortical demyelination: multiple sclerosis-like lesions with rapid resolution of inflammation and extensive remyelination. Brain (2006) 129:1972-83. doi:10.1093/brain/awl135

20. Greter M, Lelios I, Croxford AL. Microglia versus myeloid cell nomenclature during brain inflammation. Front Immunol (2015) 6:249. doi:10.3389/ fimmu.2015.00249

21. Ajami B, Bennett JL, Krieger C, McNagny KM, Rossi FM. Infiltrating monocytes trigger EAE progression, but do not contribute to the resident microglia pool. Nat Neurosci (2011) 14:1142-9. doi:10.1038/nn.2887

22. Heim N, Garaschuk O, Friedrich MW, Mank M, Milos RI, Kovalchuk $\mathrm{Y}$, et al. Improved calcium imaging in transgenic mice expressing a troponin C-based biosensor. Nat Methods (2007) 4:127-9. doi:10.1038/ nmeth 1009

Conflict of Interest Statement: The authors declare that the research was conducted in the absence of any commercial or financial relationships that could be construed as a potential conflict of interest.

Copyright (C) 2016 Radbruch, Bremer, Guenther, Cseresnyes, Lindquist, Hauser and Niesner. This is an open-access article distributed under the terms of the Creative Commons Attribution License (CC BY). The use, distribution or reproduction in other forums is permitted, provided the original author(s) or licensor are credited and that the original publication in this journal is cited, in accordance with accepted academic practice. No use, distribution or reproduction is permitted which does not comply with these terms. 


\title{
Analyzing Nicotinamide Adenine Dinucleotide Phosphate Oxidase Activation in Aging and Vascular Amyloid Pathology
}

\author{
Helena Radbruch', Ronja Mothes',2, Daniel Bremer2, Stefanie Seifert', Ralf Köhler ${ }^{3}$, \\ Julian Pohlan ${ }^{1,2}$, Lennard Ostendorf ${ }^{2}$, Robert Günther ${ }^{2}$, Ruth Leben ${ }^{2}$, Werner Stenzel', \\ Raluca Aura Niesner ${ }^{2 \dagger}$ and Anja E. Hauser ${ }^{2,4 * t}$

\begin{abstract}
${ }^{1}$ Department of Neuropathology, Charité - Universitätsmedizin Berlin, Berlin, Germany, ${ }^{2}$ German Rheumatism Research Center (DRFZ), A Leibniz Institute, Berlin, Germany, ${ }^{3}$ Immune Dynamics, Deutsches Rheuma-Forschungszentrum (DRFZ),
\end{abstract} \\ A Leibniz Institute, Berlin, Germany, ${ }^{4}$ Immune Dynamics, Charité - Universitätsmedizin Berlin, Berlin, Germany
}

\section{OPEN ACCESS}

Edited by:

Michael Hickey,

Monash University, Australia

Reviewed by:

Peter John Crack,

University of Melbourne, Australia

Carsten Berndt,

Heinrich Heine Universität Düsseldorf,

Germany

*Correspondence:

Anja E. Hauser

hauser@drf.de,

anja.hauser-hankeln@charite.de

tThese authors have contributed equally to this work.

Specialty section:

This article was submitted to Multiple Sclerosis and Neuroimmunology,

a section of the journal

Frontiers in Immunology

Received: 01 December 2016 Accepted: 04 July 2017 Published: 31 July 2017

Citation:

Radbruch H, Mothes R, Bremer D,

Seifert S, Köhler R, Pohlan J, Ostendorf $L$, Günther R, Leben $R$,

Stenzel W, Niesner RA and

Hauser AE (2017) Analyzing Nicotinamide Adenine Dinucleotide

Phosphate Oxidase Activation in

Aging and Vascular Amyloid

Pathology.

Front. Immunol. 8:844.

doi: 10.3389/fimmu.2017.00844
In aging individuals, both protective as well as regulatory immune functions are declining, resulting in an increased susceptibility to infections as well as to autoimmunity. Nicotinamide adenine dinucleotide phosphate (NADPH) oxidase 2-deficiency in immune cell subsets has been shown to be associated with aging. Using intravital marker-free $N A D(P)$ $\mathrm{H}$-fluorescence lifetime imaging, we have previously identified microglia/myeloid cells and astrocytes as main cellular sources of NADPH oxidase (NOX) activity in the CNS during neuroinflammation, due to an overactivation of NOX. The overactivated NOX enzymes catalyze the massive production of the highly reactive $\mathrm{O}_{2}^{-}$, which initiates in a chain reaction the overproduction of diverse reactive oxygen species (ROS). Agedependent oxidative distress levels in the brain and their cellular sources are not known. Furthermore, it is unclear whether in age-dependent diseases oxidative distress is initiated by overproduction of ROS or by a decrease in antioxidant capacity, subsequently leading to neurodegeneration in the CNS. Here, we compare the activation level of NOX enzymes in the cerebral cortex of young and aged mice as well as in a model of vascular amyloid pathology. Despite the fact that a striking change in the morphology of microglia can be detected between young and aged individuals, we find comparable low-level NOX activation both in young and old mice. In contrast, aged mice with the human APP ${ }^{E 693 Q}$ mutation, a model for cerebral amyloid angiopathy (CAA), displayed increased focal NOX overactivation in the brain cortex, especially in tissue areas around the vessels. Despite activated morphology in microglia, NOX overactivation was detected only in a small fraction of these cells, in contrast to other pathologies with overt inflammation as experimental autoimmune encephalomyelitis (EAE) or glioblastoma. Similar to these pathologies, the astrocytes majorly contribute to the NOX overactivation in the brain cortex during CAA. Together, these findings emphasize the role of other cellular sources of activated NOX than phagocytes not only during EAE but also in models of amyloid pathology. Moreover, they may strengthen the hypothesis that microglia/monocytes show a diminished potential for clearance of amyloid beta protein. 


\section{INTRODUCTION}

Following the free-radical theory of aging, reactive oxygen species (ROS) are massively produced, e.g., $>100 \mathrm{nM} \mathrm{H}_{2} \mathrm{O}_{2}$, and attack their targets in the organism randomly, indiscriminative and cumulative, thus, generating oxidative distress (1). Oxidative distress is a general term for the dysfunction of signaling and defense mechanisms based on ROS.

As several ROS are highly reactive, their localization is crucial for the resulting effects: on the one hand, ROS act as specific signaling molecules intracellularly at $\sim 1-10 \mathrm{nM}$ concentration, on the other hand, they constitute effective extracellular host defense mechanisms at concentrations over $100 \mathrm{nM}$. Since the organism has efficient mechanisms to neutralize high ROS concentrations, e.g., via glutathione peroxidase or superoxide dismutase (2), these phenomena are physiological and can be summarized under the term "oxidative eustress" (1). The enzymes of the nicotinamide adenine dinucleotide phosphate (NADPH) oxidase family, consisting of NOX1, NOX2 (phox), NOX3, NOX4, DUOX1, DUOX2 (3), are central players leading both to oxidative eustress and distress. Their activation catalyzes the oxidative burst when abundant highly reactive $\mathrm{O}_{2}^{-}$ is produced by oxidation of molecular oxygen. Under enzymatic catalysis, $\mathrm{O}_{2}^{-}$reacts with various small molecules leading to massive ROS production. When this massive ROS production exceeds the capacities of antioxidant defense mechanisms of the tissue or when extracellular highly reactive ROS species such as $\mathrm{H}_{2} \mathrm{O}_{2}$ enter the cells via peroxiporins such as AQP8 (4), oxidative distress occurs, leading to tissue dysfunction and damage. It is assumed that oxidative distress responsiveness is linked to aging. However, this hypothesis is mainly funded on genetic studies (5), which allow conclusions on the expression levels, but not on the actual activation of these central enzymes.

Here, we focus on NADPH oxidase enzyme activation levels in the brain, as this organ is especially vulnerable to oxidative distress. There is evidence that the aged brain is more susceptible to injuries (6). This is mainly attributed to a so-called activated basal state of low-grade chronic inflammation that has been called "inflamm-aging" (7). Low-grade inflammation in aging is also associated with microgliosis; however, the function of microglia in this process is highly discussed as studies using cell morphology, protein expression, cellular dynamics, or ex vivo cytokine production could detect age-dependent differences (8). No information on the age-dependent oxidative distress levels and their cellular sources in situ are available.

In this study, we determine NADPH oxidase activation levels in the context of amyloid pathology, in order to investigate age-dependent, immune-mediated tissue damaging mechanisms in neurodegenerative diseases. We hypothesize that an age-dependent dysregulation of immune responses in the CNS contributes to neuroinflammatory processes associated with neurodegeneration. ROS have been implicated in mediating age-dependent changes and promoting age-dependent neurodegeneration (9). Recently, oxidative distress has been regarded as an early sign of Alzheimer's disease (AD) pathophysiology, although the source of ROS and the mechanisms how amyloid peptides $(\mathrm{A} \beta)$ influence oxidative distress have not been adequately investigated (10). Subunits of NOX2 are upregulated in patients with mild cognitive impairment compared to normal age-matched controls. During disease progression, a further increase of the cytosolic subunits p67phox, p47phox, and p40phox could be detected in the CNS tissue. In addition, there was a robust correlation between NOX subunit expression and the individual's cognitive status (11). Together, this suggests that increases in NOX activity participate in early AD pathogenesis and contribute to $\mathrm{AD}$ progression due to massive ROS production initiated by NADPH oxidases, activating signaling pathways leading to neuronal excitotoxicity and glial cell-mediated inflammation (12).

Although many studies claim to analyze NOX activity, only limited information can be gained based on the analysis of subunit expression levels. The quantification of ROS levels in tissue has been widely used. However, the highly diffusive nature of ROS does not allow to draw conclusions on their origin in tissues. Up to now, neither the catalytic activity of NOX enzymes leading to oxidative distress nor their cellular sources could be tracked in vivo. We therefore use our previously published marker-free method of intravital fluorescence lifetime imaging (FLIM) of $\mathrm{NAD}(\mathrm{P}) \mathrm{H}$ (13) to analyze the distribution and cellular source of NADPH oxidase activation $(14,15)$. Since activated NOX enzymes are membrane-bound and can be detected by FLIM, this technique allows for an unambiguous identification of the cellular source of massive ROS production.

\section{MATERIALS AND METHODS}

\section{Two-Photon Laser-Scanning Microscopy}

For intravital imaging, a frontoparietal cranial window preparation was performed and the dural layer was removed according to previous publications (16). Both fluorescence intensity and FLIM experiments were performed as previously described with a two-photon laser-scanning microscope based on a commercial scan head (TriMScope, LaVision BioTec, Bielefeld, Germany). All images are acquired in 30-150 $\mu \mathrm{m}$ depth within the frontoparietal cortex $(z$-step $=2 \mu \mathrm{m})$. Ten imaging fields per mouse were acquired, and the fields of vision with the highest NOX enzyme activation in every group were included into analysis. The detection of the fluorescence signals was accomplished either with photomultiplier tubes in the ranges $460 \pm 30,525 \pm 25,593 \pm 20 \mathrm{~nm}$ or with a 16-channel parallelized TCSPC detector (FLIM-X ${ }_{16}$, LaVision BioTec, Bielefeld, Germany) in the range $460 \pm 30 \mathrm{~nm}$. The excitation of NADH and NADPH [hereafter collectively referred to as $\mathrm{NAD}(\mathrm{P}) \mathrm{H}$ ] was performed at $760 \mathrm{~nm}$ (detection at $460 \pm 30 \mathrm{~nm}$ ). Dextranrhodamine (detection at $593 \pm 20 \mathrm{~nm}$ ) and EGFP (detection at $525 \pm 25 \mathrm{~nm}$ ) were excited at 850 or $880 \mathrm{~nm}$. For intensity and FLIM, we used an average maximum laser power of $8 \mathrm{~mW}$. The experimental parameters for FLIM were 80 ps histogram bin [for $\mathrm{NAD}(\mathrm{P}) \mathrm{H}$-FLIM] and maximum acquisition time for a $512 \times 512$ image was 5 s to record a fluorescence decay stack. The time-window in which the fluorescence decays were acquired was set to 9 ns. 


\section{Data Analysis}

Fluorescence lifetime imaging data analysis was carried out with self-written software, as previously described $(14,15)$. We used a bi-exponential decay function (Eq. 1):

$$
I_{\mathrm{NAD}(\mathrm{P}) \mathrm{H}}(t)=\varepsilon+a_{1} \cdot e^{-t / \tau_{1}}+a_{2} \cdot e^{-t / \tau_{2}}
$$

with $\varepsilon$ the background, the 1 -indexed term of the sum representing the fluorescence decay of free NADH and NADPH, and the 2 -indexed term representing the fluorescence decay of enzymebound $\mathrm{NAD}(\mathrm{P}) \mathrm{H}$. The fluorescence lifetime $\tau_{1}[$ free $\mathrm{NAD}(\mathrm{P}) \mathrm{H}]$ is $400-450 \mathrm{ps}$, while the fluorescence lifetime $\tau_{2}$ of $\mathrm{NAD}(\mathrm{P}) \mathrm{H}$ bound to metabolic enzymes has an average of $\sim 2,000 \mathrm{ps}$ and of 3,650 ps if bound to an enzyme of the NOX family. We focused in this study on the fluorescence lifetime $\tau_{2}$, of the enzyme-bound $\mathrm{NAD}(\mathrm{P}) \mathrm{H}$. As previously described (14), we calculated the area of NOX activation within a $\tau_{2}$ image as the percentage of pixels having fluorescence lifetimes between 3,300 and 3,900 ps as compared to the total number of pixels displaying fluorescence lifetimes between 0 and 10,000 ps.

In order to quantify microglial ramification in young and old Iba1:GFP mice from immunofluorescence data, we used our previously published algorithm based on discrete Fourier coefficients (17). Briefly, single microglia cells were segmented from the green channel of the immunofluorescence data acquired in the cortex of healthy 6 or 20 months old Ibal:GFP mice. Their shape was approximated by overlapping circles-the first centered in the center of the cell and the others centered on the periphery of the previous circle. Each layer of circles is mathematically characterized by a scalar parameter called Fourier coefficient. The first Fourier coefficient represents the position of the cell, the second defines its dimensions by approximating it with a sphere, and the next coefficients define the degree of ramification of the cellular processes.

Statistical analysis and graphical presentation was performed with GraphPad Prism 4 (GraphPad Software, USA) and OriginPro (OriginLab, USA). Results are shown as mean values from the analyzed imaging fields $( \pm$ SD) per group.

\section{Mice}

$\mathrm{APP}^{\mathrm{E} 693 \mathrm{Q}}$ i.v. labeled with sulforhodamine 101 (for astrocyte staining in vivo) or APP ${ }^{\mathrm{E} 93 \mathrm{Q}}:$ Iba-1-EGFP mice $(18,19)$ hemizygous for both genes were generated and maintained on a C57BL/6J background. Littermates with wild-type murine APP were used as controls. Groups of $n \geq 3$ mice were used in all in vivo experiments and also for postmortem histological analysis, $n \geq 3$ mice per group were used. Young mice were 6 months old and aged mice were between 18 and 24 months old at time of analysis (in APP mutant and wildtype group; individual mouse age listed in Table 1). Animals were group housed in standard cages under pathogen-free conditions on a 12-h light/dark cycle with food and water ad libitum. All animal experiments were performed in accordance to the national animal protection guidelines approved by the regional veterinary office for health and social services in Berlin (LaGeSo Berlin).

\section{Murine Tissue Processing and Immunofluorescence Histology}

After each intravital microscopy experiment, the brains of the mice were prepared for immunofluorescence by perfusion with $4 \%$
TABLE 1 | Overview of NOX activation from individual mice analyzed.

\begin{tabular}{|c|c|c|}
\hline Mouse ID & Age (months) & NOX activation (\%) \\
\hline WT & 6 & 1.7 \\
\hline \multicolumn{3}{|l|}{ Young 1} \\
\hline WT & 6 & 1 \\
\hline \multicolumn{3}{|l|}{ Young 2} \\
\hline WT & 6 & 0.6 \\
\hline \multicolumn{3}{|l|}{ Young 3} \\
\hline WT & 24 & 0.3 \\
\hline \multicolumn{3}{|l|}{ Old 1} \\
\hline WT & 20 & 0.7 \\
\hline \multicolumn{3}{|l|}{ Old 2} \\
\hline WT & 18 & 1.5 \\
\hline \multicolumn{3}{|l|}{ Old 3} \\
\hline WT & 20 & 1.8 \\
\hline \multicolumn{3}{|l|}{ Old 4} \\
\hline APP & 6 & 0.8 \\
\hline \multicolumn{3}{|l|}{ Young } \\
\hline APP & 24 & 4.3 \\
\hline \multicolumn{3}{|l|}{ Old 1} \\
\hline APP & 24 & 4.2 \\
\hline \multicolumn{3}{|l|}{ Old 2} \\
\hline APP & 20 & 4.2 \\
\hline \multicolumn{3}{|l|}{ Old 3} \\
\hline APP & 18 & 5.2 \\
\hline \multicolumn{3}{|l|}{ Old 4} \\
\hline APP & 18 & 5.4 \\
\hline Old 5 & & \\
\hline
\end{tabular}

1-4 imaging fields were analyzed per mouse. NOX activation represents mean values.

paraformaldehyde. The tissue was embedded in Tissue Tek (Sakura), frozen in a methylbutane/dry ice mixture into $10-$ or $30-\mu \mathrm{m}$ sections using a cryostat. Sections were stained with goat anti-EGFP (Rockland, conjugated at the DRFZ to Alexa ${ }^{\circledR} 488$ ), mouse antiGFAP-Alexa ${ }^{\circledR} 488$ (eBioscience, Germany), rabbit anti-Noxo-1 (Novus Biologicals, Germany), or goat anti-p47 (Abcam, Germany). Secondary antibodies used were donkey anti-rabbit Alexa ${ }^{\circledR} 647$ (Life Technologies, Germany) or donkey anti-goat-Alexa ${ }^{\circledR} 647$. Slides were analyzed on a Zeiss LSM 710 confocal system. Co-localization was analyzed by standardized background subtraction and subsequently multiplying the Noxol and GFAP channel using with Fiji Software.

\section{Human Tissue Processing and Immunofluorescence Histology}

Brain samples were obtained from the Department of Neuropathology, Charité - Universitätsmedizin Berlin. We included only anonymized individuals with cerebral amyloid angiopathy (CAA). The study was approved by the local ethics committee under the number EA1/078/16. Paraffin-embedded brain tissues were sectioned at $4 \mu \mathrm{m}$ with a microtome (Microm HM330, GMI), followed by deparaffinization and rehydration in a decreasing ethanol series. For immunofluorescence staining, sections were blocked with $10 \%$ normal serum (NS) for $1 \mathrm{~h}$. After washing, sections were incubated with primary antibodies mouse a-hu HLA-DR 1:100 (Dako), goat a-hu p47phox 1:100 (Abcam), rabbit a-GFAP (DAKO) 1:2,000, and mouse a-oxidized phospholipids at 1:50 (clone E06, Avanti Polar Lipids) with 2\% NS. Secondary antibodies (goat a-ms-Alexa ${ }^{\circledR} 546$ and chicken a-goat-Alexa ${ }^{\circledR} 594$, donkey a-rabbitAlexa ${ }^{\circledR} 488$ ) were diluted 1:500 with $2 \%$ NS and added to the sections. Nuclei were stained with DAPI (Sigma) 
at a 1:1,000 dilution in phosphate-buffered saline. Slides were analyzed on a Zeiss LSM 710 confocal system.

\section{RESULTS}

\section{Differences in the Morphology, but Similar NADPH Oxidase Activation Levels in Cortical Microglia of Young and Old Mice}

When analyzing the morphology of microglia in Iba-1-EGFP reporter mice by immunofluorescence microscopy in various age cohorts, we noticed striking differences between young and old individuals. While the majority of microglia in mice at the age of 6 months displayed a ramified, surveilling morphology in the cortex, we noted that those cells in mice between 18 and 24 months of age had short, blunt processes, suggesting a more activated phenotype (Figure 1A). Using our previously published algorithm based on discrete Fourier coefficients (17), we found a higher ramification of the microglial processes in young mice ( $n=122$ cells $)$ than in old mice $(n=83$ cells) as indicated by all Fourier coefficients up to the $10^{\text {th }}$ rank, especially by the $3^{\text {rd }}$ Fourier coefficient (graph in Figure 1A).

The detection of these morphological differences in microglia between various young and aged mice prompted us to
A

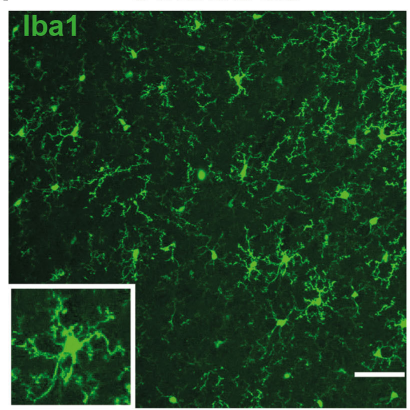

B
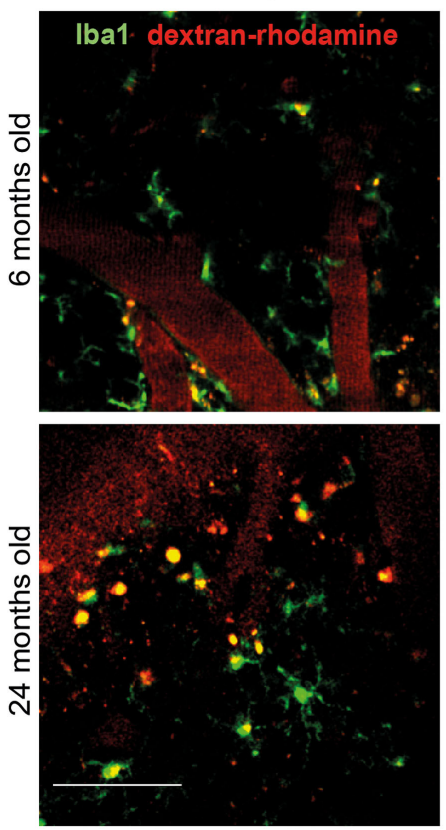

20 months old

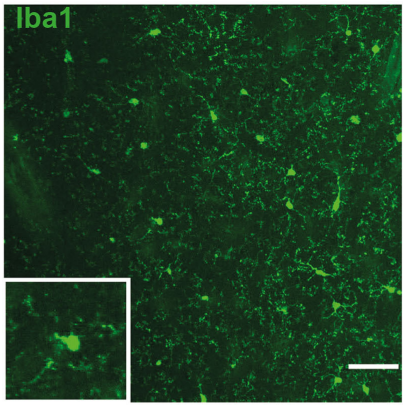

C $\quad \mathrm{NAD}(\mathrm{P}) \mathrm{H}-\mathrm{FLIM}$
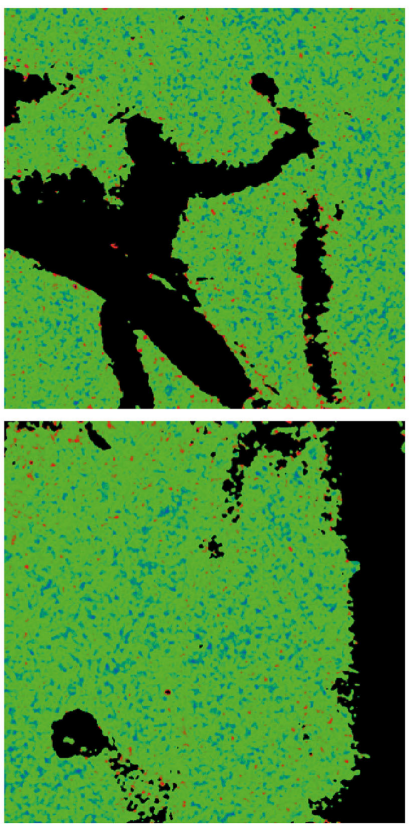

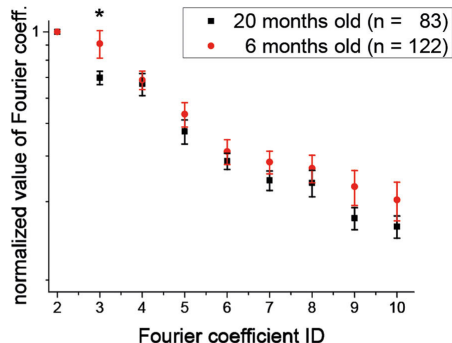

NAD(P)H-FLIM (NOX gate)
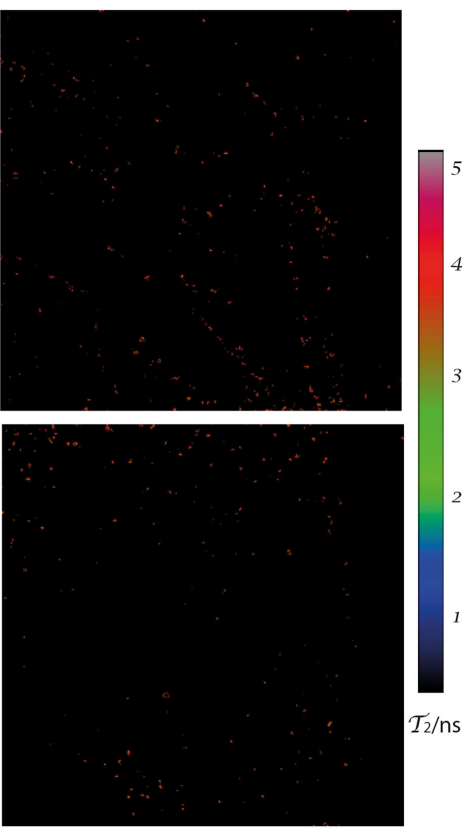

FIGURE 1 | Morphology of cortical microglia and nicotinamide adenine dinucleotide phosphate (NADPH) oxidase activation in young and old healthy mice. (A) lba-1:EGFP reporter mice were stained with anti-EGFP and 30- $\mu \mathrm{m}$ thick sections were analyzed of frontoparietal cortex by confocal microscopy. Representative immunofluorescence images (maximum intensity projections) from the neocortex of a 6-month-old (left) and 20-month-old (right) mouse indicate the differences in morphology of lba1-EGFP+ cells (green). Scale bar: $50 \mu \mathrm{m}$. In the graph, the normalized values of the $3^{\text {rd }}$ to the $10^{\text {th }}$ Fourier coefficients, calculated as described in the manuscript, indicate that the ramification of the microglial processes is higher in young $(n=4)$ than in old healthy mice $(n=4)$. (B) Representative images of PMT-based detection of fluorescence signals and (C) enzyme-bound NAD(P)H-fluorescence lifetime imaging (FLIM) maps $\left(\tau_{2}\right.$-maps) in a young (6 months; upper row) and old (24 months; lower row) mouse. Scale bar: $50 \mu \mathrm{m}$. The $\tau_{2}$-maps of the left column show the false color-encoded fluorescence lifetime $\tau$ of enzymebound $\mathrm{NAD}(\mathrm{P}) \mathrm{H}$ at each recorded pixel of the image. $\mathrm{NAD}(\mathrm{P}) \mathrm{H}$ bound to metabolic enzymes are depicted in blue and green ( $\tau_{2}$ between 1 and 3 ns), whereas NADPH bound to activated NOX enzymes appears in red ( $\tau_{2}$ between 3.3 and 3.9 ns, "NOX only" gate) is displayed in the right column of (B). 
A

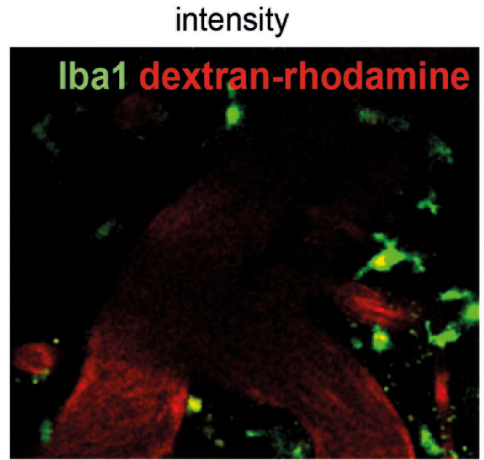

B

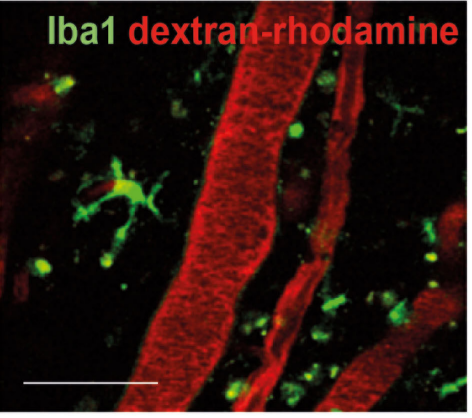

C

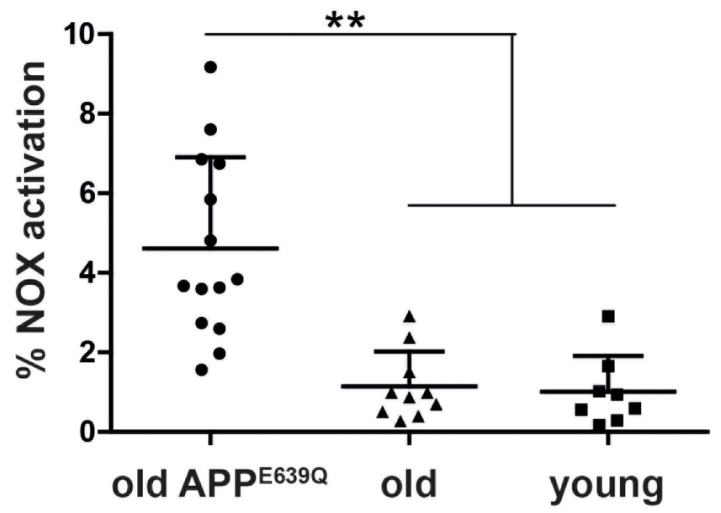

E

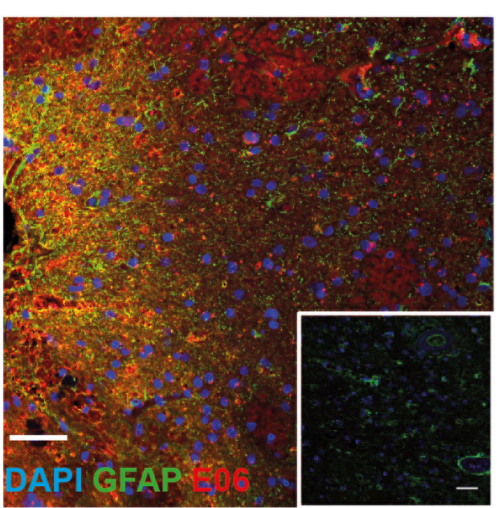

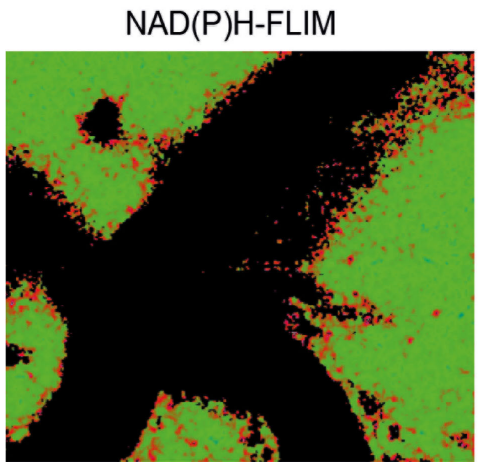

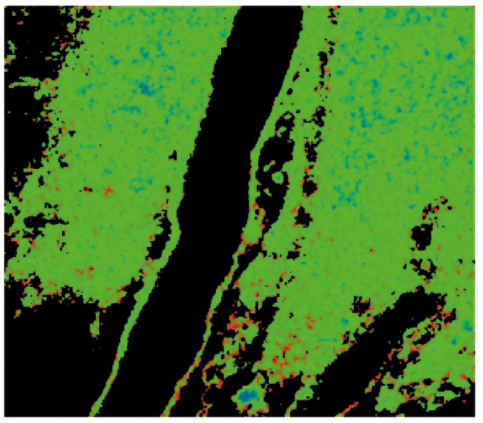

D

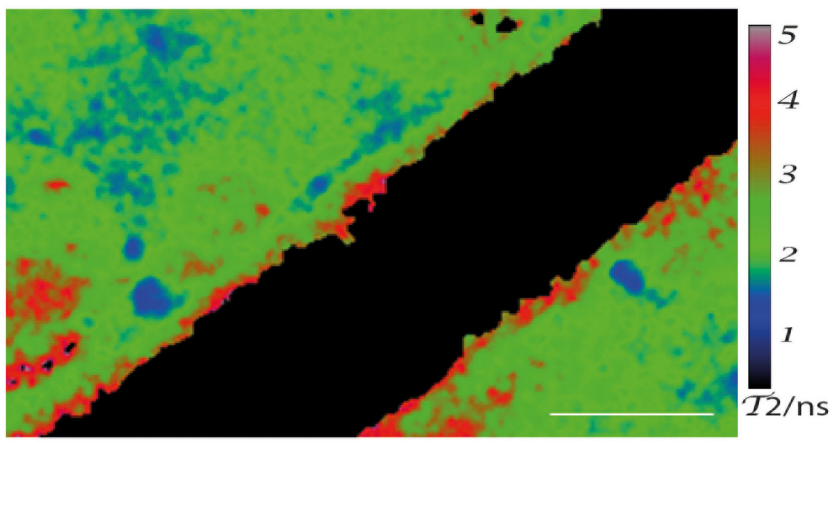

NAD(P)H-FLIM (NOX gate)
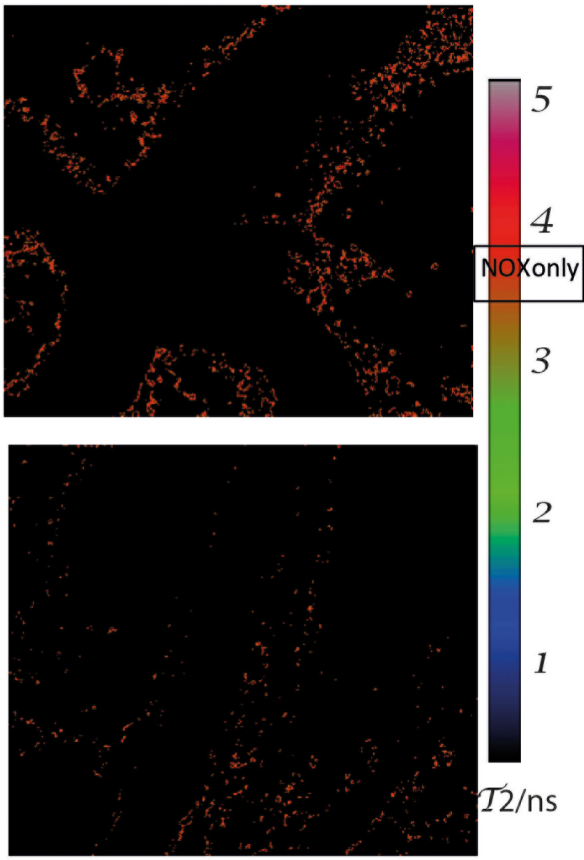

NAD(P)H-FLIM
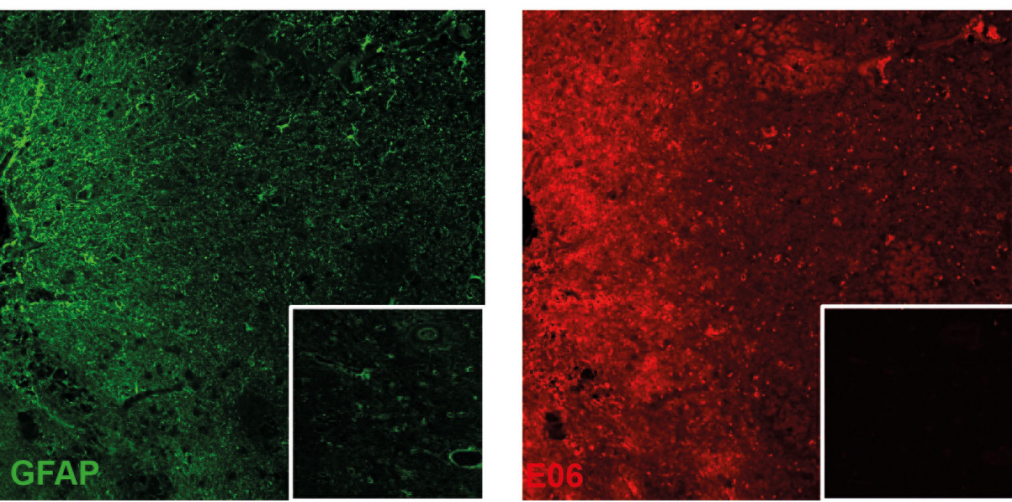
FIGURE 2 | Continued

Elevated NOX activity in the cortex of aged APPE6930 mice. Representative images of areas with elevated (A) and low (B) NOX enzymes activation are both found in old APPE693Q mice with established cerebral amyloid angiopathy (CAA). PMT-based detection of fluorescence signals [left column (A,B) dextran-rhodamine in red and iba1+ cells in green] in a 24-month-old APPE6930 mouse. Scale bar: $50 \mu \mathrm{m}$. The corresponding $\tau_{2}$-maps of the middle column show the false color-encoded fluorescence lifetime $\tau$ of enzyme-bound $\mathrm{NAD}(\mathrm{P}) \mathrm{H}$ at each recorded pixel of the image. $\mathrm{NAD}(\mathrm{P}) \mathrm{H}$ bound to metabolic enzymes are depicted in blue and green $\left(\tau_{2}\right.$ between 1 and $3 \mathrm{~ns}$ ), whereas nicotinamide adenine dinucleotide phosphate (NADPH) bound to activated NOX appears in red ( $\tau_{2}$ between 3.3 and 3.9 ns, "NOX only" gate) is displayed in the right column of (A,B). (C) Quantification of the NOX activation area within, i.e., ratio of the area of NOX only gate to the total tissue area, $4.9 \pm 2.0 \%$ ( $n=14$ fields of view of 5 mice with $2-4$ fields of view per mouse) in APPE6930 mice compared to $1.1 \pm 0.7 \%$ ( $n=10$ fields of view of 4 mice with 1-4 fields of view per mouse) and $1.0 \pm 0.9 \%$ ( $n=8$ fields of view of 3 mice with 2-3 fields of view per mouse) in healthy controls (old and young, respectively). At least 3 mice per group and 2-4 imaging fields per mouse were analyzed. For statistic evaluation, we applied the ANOVA test $\left({ }^{* *} p<0.01\right)$. (D) The $\tau 2$ NAD(P) $\mathrm{H}$-fluorescence lifetime imaging (FLIM) map shows a specific bilayered pattern in the proximity of the vasculature of APPE693Q mice. Scale bar: $25 \mu \mathrm{m}$. (E) Representative immunofluorescence image within the cortex of patients with CAA showing strong reactivity with the E06 antibody recognizing oxidized phospholipids in areas with astrogliosis (stained with anti-GFAP antibody) Scale bar: $50 \mu \mathrm{m}$, compared to normal appearing tissue from patients with CAA (shown in insets). Scale bar: $50 \mu \mathrm{m}$.

investigate whether they are actually accompanied by differences in the functionality of these cells. This was performed by making use of our previously established method of in vivo FLIM (20). The method uses a time-correlated single-photon counting device in order to detect the endogenous fluorescence lifetime of $\mathrm{NAD}(\mathrm{P}) \mathrm{H}$, as a differential indicator for the activity of metabolic enzymes as well as for NOX enzyme activation in tissue. In combination with PMT-based detection of fluorescence signals (Figure 1B), we are able to allocate NOX enzyme activation to certain neocortical regions and cell types. By applying these techniques in the neocortex of Iba1-EGFP mice injected with dextran-rhodamine, we neither detected significant differences in the overall activation state of NOXdependent enzymes nor an increase in NOX enzyme activation specifically in Iba- $1^{+}$cells in aged mice (Figure 1C). Taken together, this indicates a comparable level of metabolic activity of NOX enzymes in young and old mice.

\section{Locally Elevated NOX Activity in the Cortex of Aged APPE693Q Mice and Oxidized Phospholipids in Patients with CAA}

Next, we aimed to investigate microglia activation in an aging-associated disease. We chose an age-dependent model of congophilic A $\beta$ CAA. In this model, transgenic mice (APP ${ }^{\mathrm{E} 693 \mathrm{Q}}$ mice) overexpress E693Q-mutated human APP under the control of the neuron-specific Thyl promoter element $(19,21,22)$. Mice with the APPE693Q mutation exhibit glial reaction and neuronal apoptosis in certain areas of the brain without extensive presence of $A \beta$ plaques in the parenchyma. However, $\mathrm{APP}{ }^{\mathrm{E} 693 \mathrm{Q}}$ mice display fibrillary $\mathrm{A} \beta$ at the vessel walls leading to an amyloid angiopathy (starting at an age of 9 months), causing hemorrhagic strokes and dementia in aged mice. In order to monitor microglia activity in vivo, we crossed the $\mathrm{APP}^{\mathrm{E} 693 \mathrm{Q}}$ mice with Ibal-EGFP mice (18).

We analyzed the activation of NOX enzymes in the frontoparietal neocortex by intravital microscopy. For visualization of the vasculature, mice were injected with dextran-rhodamine prior to imaging. $\mathrm{NAD}(\mathrm{P}) \mathrm{H}$-FLIM revealed heterogeneous local elevations in NOX activity in $\mathrm{APP}^{\mathrm{E} 693 \mathrm{Q}}$ mice, and an overall elevation of NOX enzyme activity in the tissue was apparent. This effect could be mainly attributed to focal spots with elevated NOX activity (Figure 2A), which occurred near areas of normal appearing tissue with no elevation in NOX activity. Agematched control mice did not exhibit tissue regions of elevated NOX activity (Figure 2B), as reflected in the quantification of the overall percentage of NOX-signal present in the tissue (Figure 2C). The mean area of NOX enzyme activation was $4.9 \pm 2.0 \%$ ( $n=5$ mice $)$ in $\mathrm{APP}^{\mathrm{E} 693 \mathrm{Q}}$ mice older than 20 months. In contrast, aged-matched wild-type controls had only a mean of $1.1 \pm 0.9 \%$ ( $n=4$ mice) NOX enzyme activation area, a value comparable with young wild-type mice: mean $=1.0 \pm 0.9 \%$ $(n=3$ mice $)$ and with young $\mathrm{APP}^{\mathrm{E} 639 \mathrm{Q}}$ mice: mean $=0.8 \pm 0.6 \%$ ( $n=1$ mouse) (Table 1). Regarding the distribution and range, we found that elevated NOX activation was enhanced in the immediate vicinity of blood vessels of $\mathrm{APP}^{\mathrm{E} 693 \mathrm{Q}}$ mice. The activation appeared in a layered pattern around the vessels: besides the endothelium being strongly activated, we also detected other activated structures in the tissue neighboring the endothelia; frequently, these structures were found to run parallel to the vessels (Figure 2D). Having demonstrated the overactivation of NOX, we next addressed the question to what extent this NOX activity affects the CNS tissue in terms of oxidative distress. We therefore stained CNS tissue of patients with CAA by histology, using an antibody (E06) that recognizes the phosphocholine headgroup of oxidized phospholipids (23) in combination with an anti-GFAP antibody labeling astrocytes. Consistent with our results regarding elevated NOX activation in the $\mathrm{APP}^{\mathrm{E} 693 \mathrm{Q}}$ mouse model, we found a strong focal presence of oxidized phospholipids, localized in areas with an astrogliosis as displayed by GFAP immunoreactivity, compared to normal appearing tissue in patients with CAA (Figure 2E, $n=4$ ).

\section{Minor Role of Microglia As Cellular Sources of Activated NOX in Aged APP ${ }^{E 6930}$ Mice and in Patients with CAA}

In order to further identify the cellular source of NOX activity in heterozygous $\mathrm{APP}{ }^{\mathrm{E} 693 \mathrm{Q}}$ mice, we analyzed aged $\mathrm{APP}^{\mathrm{E} 693 \mathrm{Q}}$ :Iba1EGFP mice by intravital NAD(P)H-FLIM. Microglia were identified based on their EGFP fluorescence and vessels identified by the presence of dextran-rhodamine (Figure 3A, left panel). By using the EGFP-signal for masking the image of the $\mathrm{NAD}(\mathrm{P}) \mathrm{H}$-fluorescence lifetime data (Figure 3A, right panel), 
we were able to determine the levels of NOX activation specifically in microglia (Figure 3B). This "gating" strategy revealed that only a minor fraction (mean $=1.6 \pm 0.8 \% ; n=3$ mice) of the total NOX enzymes activity actually originated from microglia, whereas the major fraction arouse from other cellular sources in the parenchyma. Moreover, when we analyzed the activation state of the microglia population, we found NOX enzyme activity in only $\sim 2 \%$ of these cells (mean $=1.7 \pm 1.4 \%$; $n=3$ mice, see Table 1). Taken together, these results indicate a minor relevance of microglia in the generation of ROS in the $\mathrm{APP}^{\mathrm{E} 693 \mathrm{Q}}$ mouse model and imply other, yet undefined, cells as main contributors.

Similar to our results regarding microglial NOX activation in the $\mathrm{APP}^{\mathrm{E} 693 \mathrm{Q}}$ mouse model, we found only low expression levels of p47 (a cytosolic subunit present in all NOX enzymes) in cells with microglial morphology as compared to blood-derived immune cells such as neutrophil granulocytes or macrophages/monocytes (Figure 3C, $n=4$ ) in patients with CAA.

\section{Major Contribution of Astrocytes to Activated NOX in Aged APP ${ }^{6930}$ Mice}

Using immunofluorescence histology and intravital NAD(P) H-FLIM, we previously found NOX1-expressing astrocytes as contributors to NOX enzyme overactivation in the context of chronic neuroinflammation (17). Based on that, we hypothesized that astrocytes may also contribute to NOX enzyme activation observed in $\mathrm{APP}^{\mathrm{E} 693 \mathrm{Q}}$ mice. In order to identify further cellular sources of NOX in the cortex of these mice, we performed intravital NAD(P)H-FLIM after local staining with sulforhodamine 101 (SR101). Astrocytes were identified based on their SR101 fluorescence (Figure 4A, left panel), which was used to mask the corresponding $\mathrm{NAD}(\mathrm{P}) \mathrm{H}$-fluorescence lifetime maps (Figure 4B, left panel) and, thus, to determine the NOX activation levels in these cells (Figure 4B, right panel). By "gating" the NAD (P)H-FLIM maps in this way, we identified SR101 labeled cells (astrocytes) to be major cellular contributors to the NOX activation signal in the cortex of aged APP $\mathrm{AP}^{\mathrm{E} 93}$ mice $($ mean $=37.9 \pm 2.1 \% ; n=2$ mice $)$.

In order to verify this finding using immunofluorescence histology, we stained brain cortex sections from aged $\mathrm{APP}{ }^{\mathrm{E} 693 \mathrm{Q}}$ mice with antibodies against the Noxo1, a membrane-bound subunit of NOX1. While NOX2 is mainly expressed in phagocytic cells, other NOX enzymes prevail in other cell types $(3,17)$. Consistent with our intravital data, we could detect an enriched membrane-bound Noxo1 signal, co-localizing with processes of astrocytes (Figures 4C,D). Taken together, our data suggest that the observed bi-laminar NOX activation pattern in $\mathrm{APP}^{\mathrm{E} 693 \mathrm{Q}}$ mice consists of an inner endothelial NOX activation and an outer astrocytic NOX activation layer.

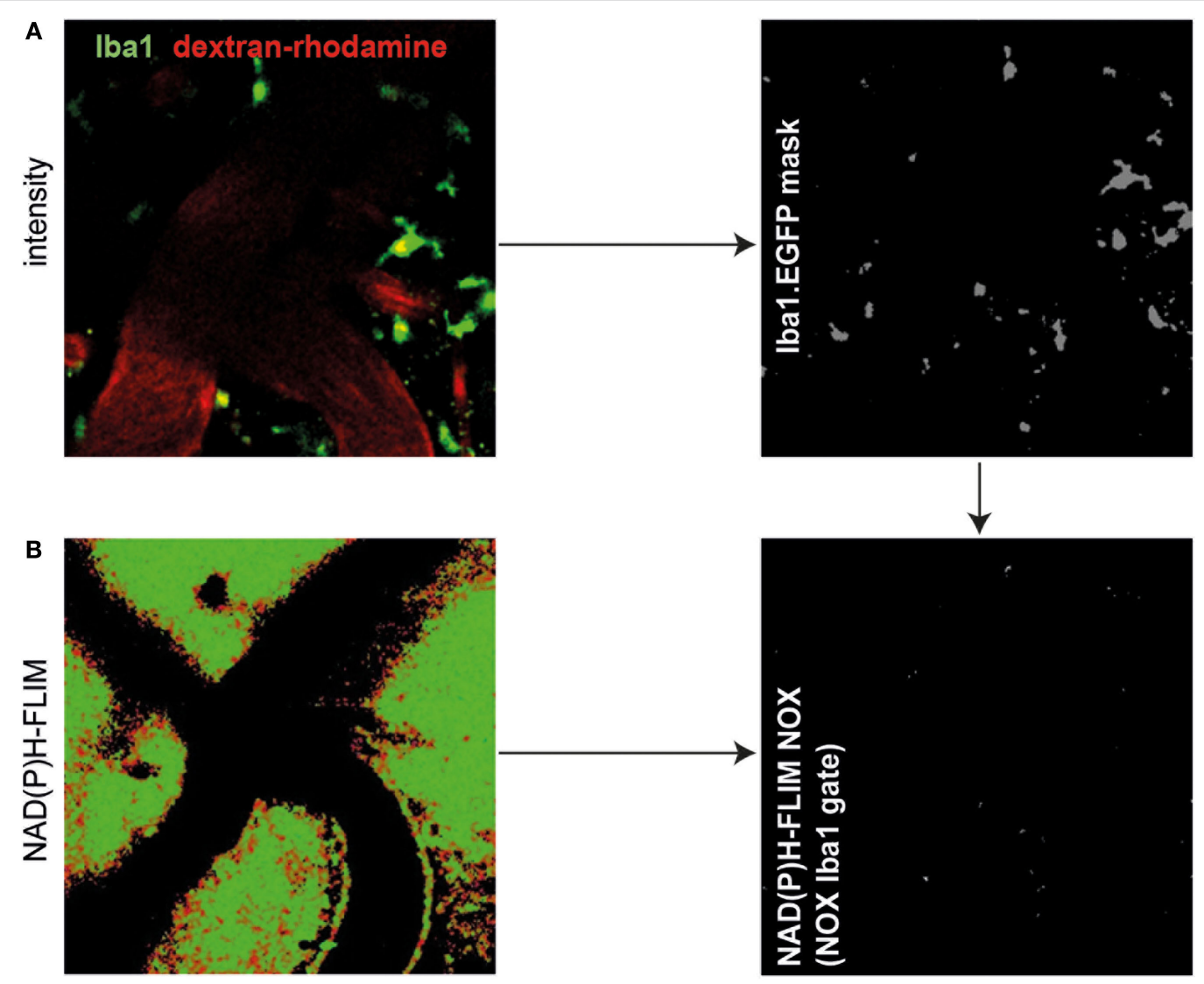

FIGURE 3 | Continued 

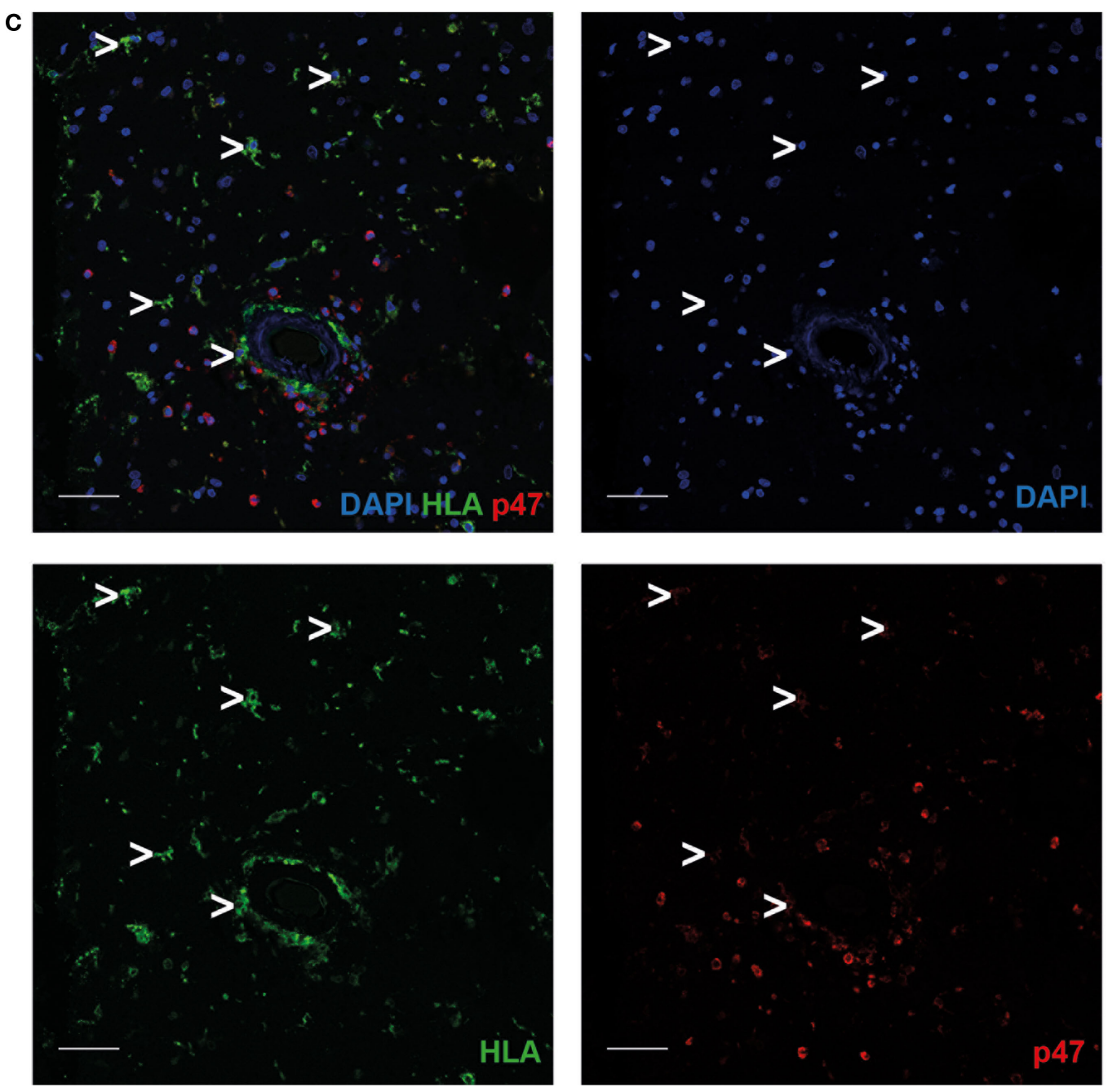

FIGURE 3 | Minor role of microglia as cellular sources of activated NOX in APPE693Q mice and patients with cerebral amyloid angiopathy (CAA). Fluorescence intensity images of the cortex of a lba1-EGFP-APPE6930 mouse (A) and corresponding $\tau_{2} \mathrm{NAD}(\mathrm{P}) \mathrm{H}$-fluorescence lifetime imaging (FLIM) maps of the whole tissue area (B) were compared to determine the contribution of microglia to the NOX activation signal. We performed an overlay of the two images, as in (A,B), and analyzed the $\mathrm{NAD}(\mathrm{P}) \mathrm{H}$-FLIM signal at the areas of the $\mathrm{lba}-1^{+}$cells (green). The $\tau_{2} \mathrm{NAD}(\mathrm{P}) \mathrm{H}-\mathrm{FLIM}$ images depict the normalized area of $\mathrm{NOX}$ activation in relation to the total cellular area in the lba- $1^{+}$cell subsets: mean $1.6 \pm 0.8 \%$ out of 3 mice; (2-4 fields of view per mouse). Scale bar: $50 \mu$ m. (C) Representative immunofluorescence image within the cortex of patients with CAA showing enriched p47 at the membrane of blood-derived innate immune cells but not in cells with microglial morphology (white arrowheads) ( $n=4$ patients. Scale bar: $50 \mu \mathrm{m})$.

\section{DISCUSSION}

Age-related changes in immune responses, commonly known as immunosenescence, are increasingly moving in the focus of immunological research. Immunosenescence is a multifactorial phenomenon caused, among other factors, by the diminished potential of hematopoietic stem cells to self-renew (24), agedependent impairment of antigen-presenting cells (25), a reduction of $\mathrm{T}$ cell (26) and B cell repertoire and numbers (27), causing a decline in adaptive immunity (28). A reduction in numbers of phagocytes has been described in aged individuals (29), along with an impaired functionality, which has been suggested to reflect the adaptation to age-associated changes in their environment (30).
On a molecular level, an age-related deficiency in NOX2 has been recently shown to result in a reduced suppressive function in aged $\mathrm{CD}^{+}$regulatory $\mathrm{T}$ cells (31), leading to inflammation and tissue destruction. Whether changes in NOX activity also occur in other immune subsets during aging, especially in phagocytic cells, which are known to express NOX2 at high levels, is not known. By analyzing the changes in fluorescence lifetime of $\mathrm{NAD}(\mathrm{P}) \mathrm{H}$ as a means to analyze NOX activation in vivo, we recently demonstrated that NOX enzyme overactivation in cerebral phagocytes contributes to oxidative distress associated with chronic neuroinflammation. Here, we compared the activation of microglia in young versus aged mouse cohorts. We found a striking difference in microglia morphology between the two groups. While the majority of those cells 

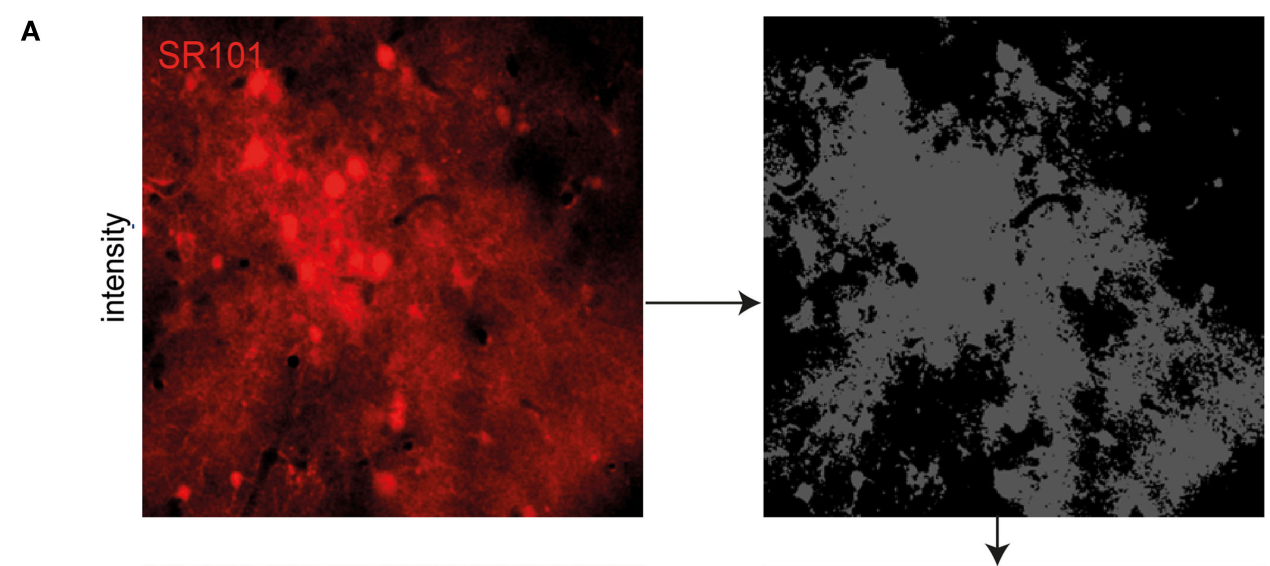

B
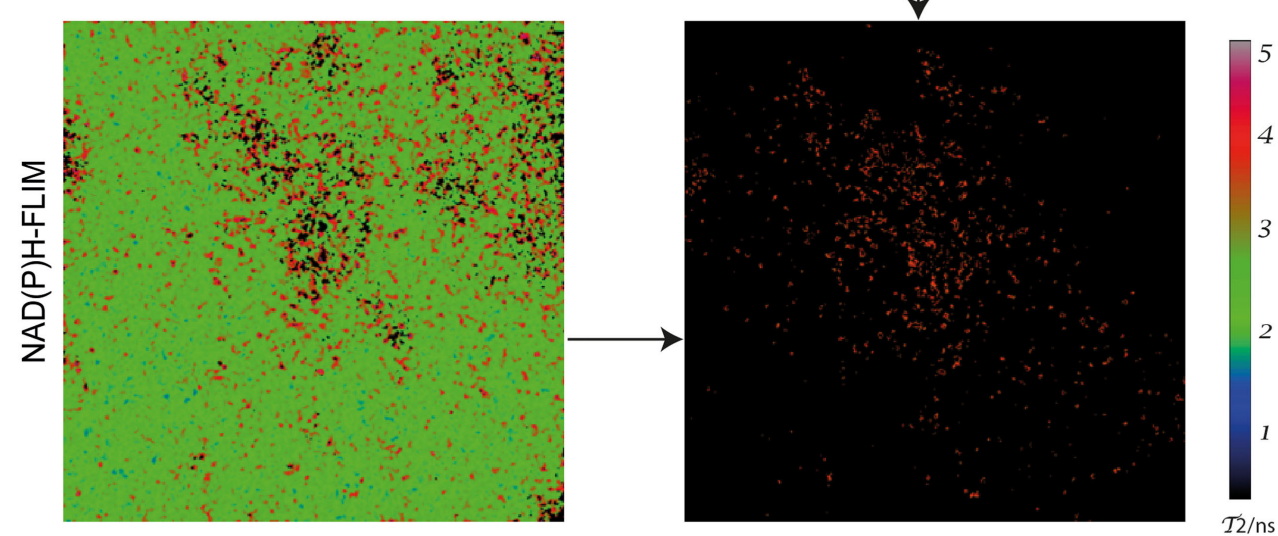

C
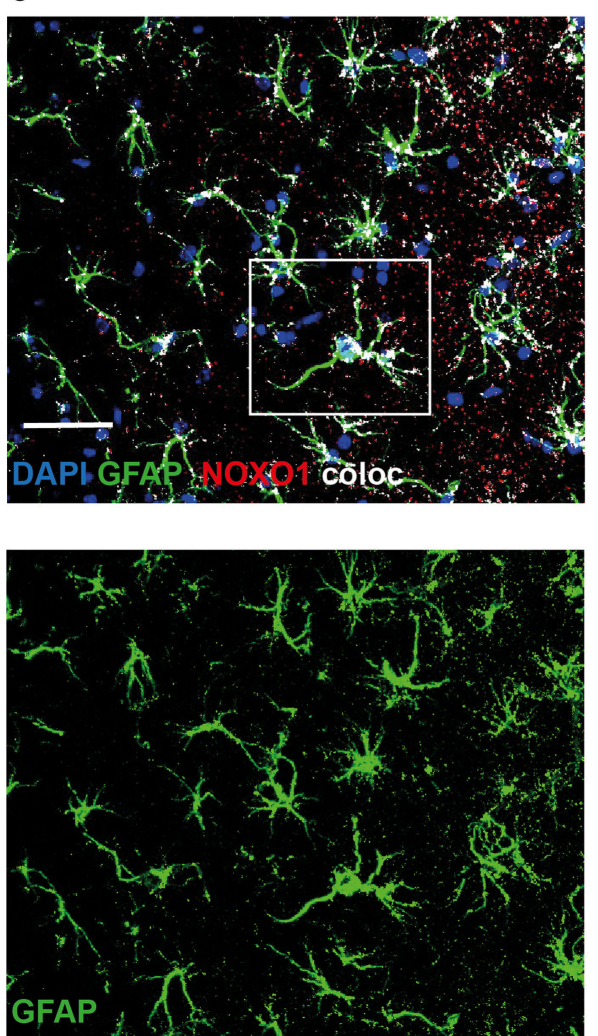

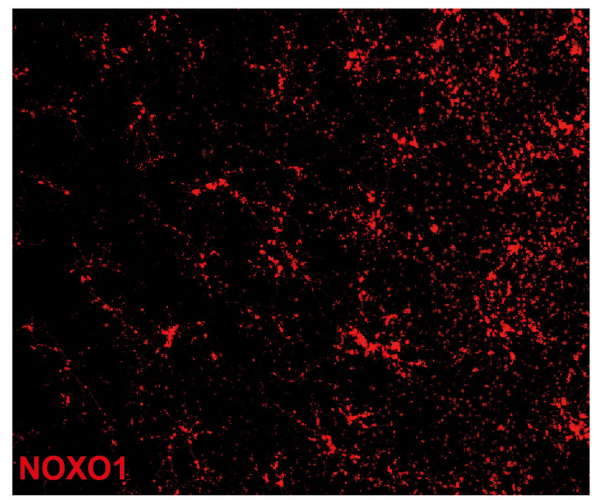

D
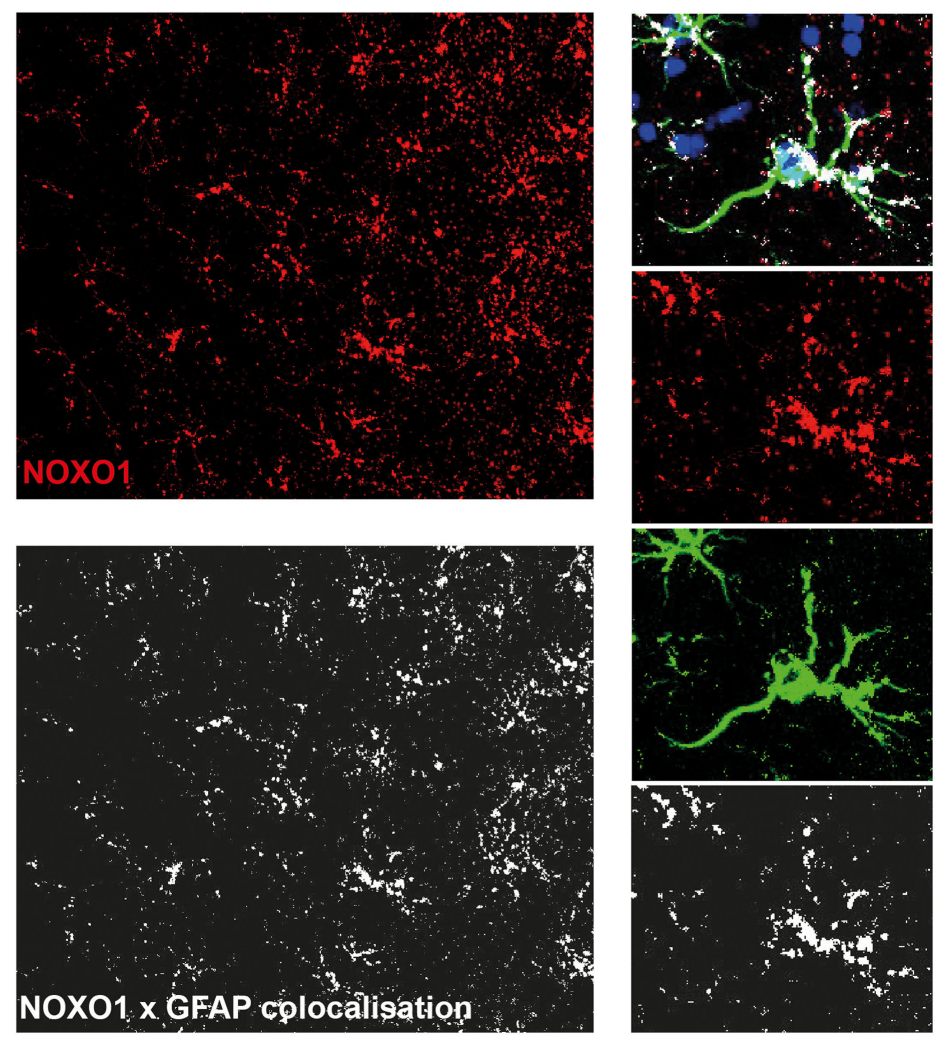
FIGURE 4 | Continued

Astrocytes are major cellular sources of activated NOX in APPE693Q mice. Similar to Figure 3, fluorescence intensity images of the cortex of a APPE693Q mice labeled with sulforhodamine 101 (SR101) (A) and corresponding $\tau_{2} \mathrm{NAD}(\mathrm{P}) \mathrm{H}$-fluorescence lifetime imaging (FLIM) maps of the whole tissue area (B) were correlatively analyzed to measure the contribution of SR101+ cells (mainly astrocytes) to the NOX activation signal. We performed an overlay of the two images and analyzed the $\mathrm{NAD}(\mathrm{P}) \mathrm{H}$-FLIM signal at the areas of SR101 signal (red). The $\tau_{2} \mathrm{NAD}(\mathrm{P}) \mathrm{H}-\mathrm{FLIM}$ images depict the normalized area of NOX activation in relation to the total cellular area in the SR101 labeled cell subsets: mean $37.9 \pm 2.1 \%$ out of 2 mice; with $2-3$ fields of view per mouse. Scale bar: $50 \mu$ m. (C) Representative immunofluorescence image within the cortex of a 20-month-old APPE693Q mouse indicating the distribution of Noxo1 (subunit of NOX1, red) and GFAP signal (green). Nuclei are stained with DAPI (blue). Scale bar: $50 \mu \mathrm{m}$. White box marks inset displayed in (D).

in young mice showed a ramified morphology typical of a surveilling behavior, microglia in old mice typically exhibited an activated phenotype characterized by shorter processes. Our findings are in line with a previous report in mice (32), describing similar changes in morphology. Moreover, a recent study has confirmed similar age-dependent changes in human microglia (33).

However, none of the previous studies functionally analyzed microglia in different age cohorts in vivo. We chose to take NOX enzyme activation as a functional readout for microglia activation. Despite the obvious differences in morphology, we could not detect differences between young and old individuals, when comparing NOX activation by NAD(P)H-FLIM in the cerebral cortex of mice. In old and young healthy mice, there was also no elevated NOX enzyme activation in the CNS tissue, arguing against a chronic low-grade tissue inflammation, which has been associated with immunosenescence, at least in microglia.

Next, we aimed to investigate NOX activation in cerebral phagocytes during age-related neurodegenerative disease. $\mathrm{AD}$, a fatal neurodegenerative condition, is one of the most common causes of dementia in aged. Major pathological changes are the deposition of extracellular amyloid $\beta(\mathrm{A} \beta)$ peptide plaques and formation of neurofibrillary tangles, which consist of the microtubule protein tau, in the CNS tissue. In the patients, neuronal dysfunction progressively leads to cognitive impairment and loss of memory. While extensive research has been performed to elucidate the role of amyloid deposits in $\mathrm{AD}$, the role of inflammation in the brain has only recently become appreciated. Microglia are in the focus of interest, especially during disease progression. A $\beta$ not only aggregates in neuronal parenchyma but can also accumulate on blood vessel walls, this condition is named CAA and can cause hemorrhagic strokes and dementia. We found elevated levels of oxidized phospholipids by immunofluorescence in cerebral tissue of patients with CAA, suggesting ongoing tissue damage by oxidative distress in areas with pronounced astrogliosis. We used a mouse model of CAA in order to analyze NOX enzyme activity in vivo. In line with the cerebral angiopathy prevailing in this mouse model, we found a strong activation of NOX in tissue areas around the blood vessels in a typical bilayered pattern. However, our analyses did not show significant elevated NOX enzyme activity in microglia, but rather in other CNS cells such as astrocytes. We could confirm this hypothesis by performing intravital $\mathrm{NAD}(\mathrm{P}) \mathrm{H}-\mathrm{FLIM}$ in $\mathrm{APP} \mathrm{E}^{\mathrm{E} 39 \mathrm{Q}}$ mice in which astrocytes were labeled by sulforhodamine 101, in which we found that more than a third of overactivated NOX enzymes are associated with astrocytes. By histology, the expression of the NOX1-subunit Noxo1 was partly localized in $\mathrm{GFAP}^{+}$cells, underlining the role of astrocytes as contributors to the elevated NOX enzyme activity in the tissue. Further cellular sources of NOX enzyme activation may include endothelial cells that have been shown to express NOX4 (34) or pericytes and vascular smooth muscle cells. However, these results extend our previous findings, which identified astrocytes as main source of oxidative stress in chronic neuroinflammation, especially when peripheral immune cells were not abundant in the CNS parenchyma during later stages of the disease (17). It should be noted that the level of NOX activation measured in the CAA model is around three times lower than in experimental autoimmune encephalomyelitis (EAE) (14) and even more in a murine glioblastoma model (16), where up to one-third of the CNS tissue shows NOX activity. This finding supports the idea of a rather low-grade chronic process occurring during neurodegenerative diseases, in contrast to an overt inflammatory response. However, a continued-rather low-level-ROS production over time can exceed the anti-oxidative capacity of the tissue and lead to a slow, but progressive tissue destruction. Hence, we expect the ROS production in CAA to be in the pathologic range, i.e., $>100 \mathrm{nM}$, but rather low as compared to the ROS concentrations found in chronic neuroinflammation of $\sim 200 \mu \mathrm{M}$ (14). Intravital NAD(P)H-FLIM reveals parallels in the mechanisms between autoimmune-mediated chronic neuroinflammation and primary neurodegenerative diseases. Our data point to astrocytes as important cellular players, which are able to maintain a state of local low-grade inflammation in the CNS tissue via extracellular ROS production. It should be emphasized at this point that by detecting NOX activity, our method is not suitable for measuring intracellular ROS production $(\sim 1-10 \mathrm{nM})$, e.g., resulting from free radicals generated in the mitochondrial respiratory chain. Rather, it focuses on the massive (physiologic and pathologic) production of extracellular ROS, for which the NADPH oxidase family plays a central role.

Interestingly, the specific pattern of NOX activation we found in the tissue of the CAA model differed from the one we had previously detected in lesions of mice with EAE or glioma. A specific NOX enzyme signal was present in the endothelial layer as well as in the vicinity of the vasculature. However, the latter did not originate from microglia in aged $\mathrm{APP}^{\mathrm{E} 693 \mathrm{Q}}$ mice. There is increasing evidence in the literature that $\mathrm{A} \beta$ causes an impairment of microglia function (35). It remains to be tested whether our findings can be extended to other $A \beta$ aggregation forms as seen, e.g., in plaques and to other microglial functions. 
In addition, it will be important to address the question if locally enhanced oxidative distress precedes the formation of $A \beta$ aggregates in the tissue, or if it is a consequence of this process.

\section{ETHICS STATEMENT}

This study was carried out in accordance with the recommendations of the Landesamt for Gesundheit and Soziales, Berlin, Germany. The protocol was approved by the Landesamt für Gesundheit und Soziales, Berlin, Germany.

\section{AUTHOR CONTRIBUTIONS}

DB, HR, JP, RN, RM, RL, and SS designed and performed research and analyzed data. RM, HR, RN, and $\mathrm{AH}$ wrote the manuscript. $\mathrm{HR}$ and $\mathrm{AH}$ initiated, organized, and supervised the project. SS provided animals and expertise in animal models with APP pathology. HR, RG, and AH provided expertise in mouse handling and intravital imaging. WS, RM, LO, JP, and AH collected

\section{REFERENCES}

1. Sies H. Hydrogen peroxide as a central redox signaling molecule in physiological oxidative stress: oxidative eustress. Redox Biol (2017) 11:613-9. doi:10.1016/j.redox.2016.12.035

2. Amir Aslani B, Ghobadi S. Studies on oxidants and antioxidants with a brief glance at their relevance to the immune system. Life Sci (2016) 146:163-73. doi:10.1016/j.lfs.2016.01.014

3. Bedard K, Krause KH. The NOX family of ROS-generating NADPH oxidases: physiology and pathophysiology. Physiol Rev (2007) 87(1):245-313. doi:10.1152/physrev.00044.2005

4. Sies $\mathrm{H}$. Role of metabolic $\mathrm{H}_{2} \mathrm{O}_{2}$ generation: redox signaling and oxidative stress. J Biol Chem (2014) 289(13):8735-41. doi:10.1074/jbc.R113.544635

5. Gemma C, Vila J, Bachstetter A, Bickford PC. Oxidative stress and the aging brain: from theory to prevention. In: Riddle DR, editor. Brain Aging: Models, Methods, and Mechanisms. Boca Raton, FL: CRC Press/Taylor \& Francis (2007).

6. Popa-Wagner A, Buga AM, Kokaia Z. Perturbed cellular response to brain injury during aging. Ageing Res Rev (2011) 10(1):71-9. doi:10.1016/j. arr.2009.10.008

7. Franceschi C, Bonafe M, Valensin S, Olivieri F, De Luca M, Ottaviani E, et al. Inflamm-aging. An evolutionary perspective on immunosenescence. Ann N Y Acad Sci (2000) 908:244-54. doi:10.1111/j.1749-6632.2000.tb06651.x

8. Wu Z, Yu J, Zhu A, Nakanishi H. Nutrients, microglia aging, and brain aging. Oxid Med Cell Longev (2016) 2016:7498528. doi:10.1155/2016/7498528

9. Finkel T, Holbrook NJ. Oxidants, oxidative stress and the biology of ageing. Nature (2000) 408(6809):239-47. doi:10.1038/35041687

10. Massaad CA. Neuronal and vascular oxidative stress in Alzheimer's disease. Curr Neuropharmacol (2011) 9(4):662-73. doi:10.2174/157015911798376244

11. Ansari MA, Scheff SW. NADPH-oxidase activation and cognition in Alzheimer disease progression. Free Radic Biol Med (2011) 51(1):171-8. doi:10.1016/j.freeradbiomed.2011.03.025

12. Simonyi A, He Y, Sheng W, Sun AY, Wood WG, Weisman GA, et al. Targeting NADPH oxidase and phospholipases A2 in Alzheimer's disease. Mol Neurobiol (2010) 41(2-3):73-86. doi:10.1007/s12035-010-8107-7

13. Niesner R, Narang P, Spiecker H, Andresen V, Gericke KH, Gunzer M. Selective detection of NADPH oxidase in polymorphonuclear cells by means of NAD(P)H-based fluorescence lifetime imaging. J Biophys (2008) 2008:602639. doi:10.1155/2008/602639

14. Mossakowski AA, Pohlan J, Bremer D, Lindquist R, Millward JM, Bock M, et al. Tracking CNS and systemic sources of oxidative stress during the course of chronic neuroinflammation. Acta Neuropathol (2015) 130(6):799-814. doi:10.1007/s00401-015-1497-x the human samples and analyzed human data. RN, RK, DB, and RL developed algorithms and analyzed data.

\section{ACKNOWLEDGMENTS}

We kindly thank P. Mex and R. Uecker for excellent technical assistance. We are grateful to F. Heppner who provided insight and fruitful discussions and as well as R. Lindquist for kindly proofreading of the manuscript.

\section{FUNDING}

We acknowledge the Deutsche Forschungsgemeinschaft under grant TRR130, (TP 17 to AH and HR, C01 to RN and AH). AH received funding under DFG HA 5354/8-1. RN was funded by DFG NI1167/3-1. AH and RN are members of the DFG FOR2165 (HA5354/6-1 and NI1167/4-1, respectively). This work was partially funded by the cluster of Excellence NeuroCure (EXC257) to $\mathrm{AH}$ and a BIH Medical Student Research Stipend to RM and JP.

15. Bremer D, Leben R, Mothes R, Radbruch H, Niesner R. Method to detect the cellular source of over-activated NADPH oxidases using NAD $(\mathrm{P}) \mathrm{H}$ fluorescence lifetime imaging. Curr Protoc Cytom (2017) 80:9.52.1-14. doi:10.1002/ cpcy. 20

16. Bayerl SH, Niesner R, Cseresnyes Z, Radbruch H, Pohlan J, Brandenburg S, et al. Time lapse in vivo microscopy reveals distinct dynamics of microgliatumor environment interactions - a new role for the tumor perivascular space as highway for trafficking microglia. Glia (2016) 64(7):1210-26. doi:10.1002/ glia. 22994

17. Radbruch H, Bremer D, Guenther R, Cseresnyes Z, Lindquist R, Hauser AE, et al. Ongoing oxidative stress causes subclinical neuronal dysfunction in the recovery phase of EAE. Front Immunol (2016) 7:92. doi:10.3389/ fimmu.2016.00092

18. Hirasawa T, Ohsawa K, Imai $\mathrm{Y}$, Ondo $\mathrm{Y}$, Akazawa $\mathrm{C}$, Uchino $\mathrm{S}$, et al. Visualization of microglia in living tissues using Iba1-EGFP transgenic mice. J Neurosci Res (2005) 81(3):357-62. doi:10.1002/jnr.20480

19. Gandy S, Simon AJ, Steele JW, Lublin AL, Lah JJ, Walker LC, et al. Days to criterion as an indicator of toxicity associated with human Alzheimer amyloid-beta oligomers. Ann Neurol (2010) 68(2):220-30. doi:10.1002/ana.22052

20. Radbruch H, Bremer D, Mothes R, Gunther R, Rinnenthal JL, Pohlan J, et al. Intravital FRET: probing cellular and tissue function in vivo. Int $\mathrm{J} \mathrm{Mol} \mathrm{Sci}$ (2015) 16(5):11713-27. doi:10.3390/ijms160511713

21. Herzig MC, Winkler DT, Burgermeister P, Pfeifer M, Kohler E, Schmidt SD, et al. Abeta is targeted to the vasculature in a mouse model of hereditary cerebral hemorrhage with amyloidosis. Nat Neurosci (2004) 7(9):954-60. doi:10.1038/nn1302

22. Kumar-Singh S, Dewachter I, Moechars D, Lubke U, De Jonghe C, Ceuterick C, et al. Behavioral disturbances without amyloid deposits in mice overexpressing human amyloid precursor protein with Flemish (A692G) or Dutch (E693Q) mutation. Neurobiol Dis (2000) 7(1):9-22. doi:10.1006/ nbdi.1999.0272

23. Schuh C, Wimmer I, Hametner S, Haider L, Van Dam AM, Liblau RS, et al. Oxidative tissue injury in multiple sclerosis is only partly reflected in experimental disease models. Acta Neuropathol (2014) 128(2):247-66. doi:10.1007/ s00401-014-1263-5

24. Ito K, Hirao A, Arai F, Matsuoka S, Takubo K, Hamaguchi I, et al. Regulation of oxidative stress by ATM is required for self-renewal of haematopoietic stem cells. Nature (2004) 431(7011):997-1002. doi:10.1038/nature02989

25. Gupta S. Role of dendritic cells in innate and adaptive immune response in human aging. Exp Gerontol (2014) 54:47-52. doi:10.1016/j.exger.2013.12.009

26. Naylor K, Li G, Vallejo AN, Lee WW, Koetz K, Bryl E, et al. The influence of age on T cell generation and TCR diversity. J Immunol (2005) 174(11):7446-52. doi:10.4049/jimmunol.174.11.7446 
27. Gibson KL, Wu YC, Barnett Y, Duggan O, Vaughan R, Kondeatis E, et al. B-cell diversity decreases in old age and is correlated with poor health status. Aging Cell (2009) 8(1):18-25. doi:10.1111/j.1474-9726.2008.00443.x

28. Weng NP. Aging of the immune system: how much can the adaptive immune system adapt? Immunity (2006) 24(5):495-9. doi:10.1016/j.immuni.2006.05.001

29. Lord JM, Butcher S, Killampali V, Lascelles D, Salmon M. Neutrophil ageing and immunesenescence. Mech Ageing Dev (2001) 122(14):1521-35. doi:10.1016/S0047-6374(01)00285-8

30. Stout RD, Suttles J. Immunosenescence and macrophage functional plasticity: dysregulation of macrophage function by age-associated microenvironmental changes. Immunol Rev (2005) 205:60-71. doi:10.1111/j.01052896.2005.00260.x

31. Weyand CM, Yang Z, Goronzy JJ. T-cell aging in rheumatoid arthritis. Curr Opin Rheumatol (2014) 26(1):93-100. doi:10.1097/BOR.0000000000000011

32. Hefendehl JK, Neher JJ, Suhs RB, Kohsaka S, Skodras A, Jucker M. Homeostatic and injury-induced microglia behavior in the aging brain. Aging Cell (2014) 13(1):60-9. doi:10.1111/acel.12149

33. Davies DS, Ma J, Jegathees T, Goldsbury AC. Microglia show altered morphology and reduced arborisation in human brain during aging and Alzheimer's disease. Brain Pathol (2016). doi:10.1111/bpa.12456
34. Schroder K, Zhang M, Benkhoff S, Mieth A, Pliquett R, Kosowski J, et al. Nox4 is a protective reactive oxygen species generating vascular NADPH oxidase. Circ Res (2012) 110(9):1217-25. doi:10.1161/ CIRCRESAHA.112.267054

35. Heppner FL, Ransohoff RM, Becher B. Immune attack: the role of inflammation in Alzheimer disease. Nat Rev Neurosci (2015) 16(6):358-72. doi:10.1038/ nrn3880

Conflict of Interest Statement: The authors declare that the research was conducted in the absence of any commercial or financial relationships that could be construed as a potential conflict of interest.

Copyright (C) 2017 Radbruch, Mothes, Bremer, Seifert, Köhler, Pohlan, Ostendorf, Günther, Leben, Stenzel, Niesner and Hauser. This is an open-access article distributed under the terms of the Creative Commons Attribution License (CC BY). The use, distribution or reproduction in other forums is permitted, provided the original author(s) or licensor are credited and that the original publication in this journal is cited, in accordance with accepted academic practice. No use, distribution or reproduction is permitted which does not comply with these terms. 
OPEN ACCESS

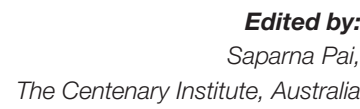

Reviewed by:

Samar S. Ayache,

Université Paris-Est, France

Julianne Teresa Djordjevic,

University of Sydney, Australia Luis R. Martinez,

New York Institute of Technology, USA

*Correspondence: Meiqing Shi mshi@umd.edu;

Christopher H. Mody cmody@ucalgary.ca

Specialty section: This article was submitted to Multiple Sclerosis and

Neuroimmunology,

a section of the journal

Frontiers in Immunology

Received: 15 April 2016

Accepted: 19 July 2016

Published: 02 August 2016

Citation:

Shi M and Mody CH (2016)

Fungal Infection in the Brain:

What We Learned from

Intravital Imaging.

Front. Immunol. 7:292.

doi: 10.3389/fimmu.2016.00292

\section{Fungal Infection in the Brain: What We Learned from Intravital Imaging}

\author{
Meiqing Shi ${ }^{* *}$ and Christopher H. Mody ${ }^{2,3 *}$ \\ ${ }^{1}$ Division of Immunology, Virginia-Maryland Regional College of Veterinary Medicine, University of Maryland, College Park, \\ MD, USA, ${ }^{2}$ Department of Microbiology, Immunology and Infectious Diseases, University of Calgary, Calgary, AB, Canada, \\ ${ }^{3}$ Department of Internal Medicine, University of Calgary, Calgary, AB, Canada
}

Approximately 1.2 billion people suffer from fungal diseases worldwide. Arguably, the most serious manifestation occurs when pathogenic fungi infect the brain, often causing fatal meningoencephalitis. For most fungi, infection occurs via the vascular route. The organism must first be arrested in the brain microvasculature and transmigrate into the brain parenchyma across the blood-brain barrier. As a result, host immune cells are recruited into the brain to contain the fungi. However, it remains poorly understood how fungi traffic to, and migrate into the brain and how immune cells interact with invading fungi in the brain. A new era of intravital fluorescence microscopy has begun to provide insights. We are able to employ this powerful approach to study dynamic interactions of disseminating fungi with brain endothelial cells as well as resident and recruited immune cells during the brain infection. In this review, with a focus on Cryptococcus neoformans, we will provide an overview of the application of intravital imaging in fungal infections in the brain, discuss recent findings and speculate on possible future research directions.

Keywords: intravital imaging, fungi, brain migration, immune cells, meningoencephalitis, $C$. neoformans

\section{INTRODUCTION}

Infectious meningitis and encephalitis are a major threat to human health, causing high mortality and morbidity throughout the world (1). Following infections, microbes including viruses, bacteria, fungi, and parasites can disseminate from sites of initial infection to the bloodstream. The circulating pathogens become arrested in the brain vasculature, followed by transmigration into the brain parenchyma across the blood-brain barrier (BBB). The BBB is a structural and functional barrier, which maintains the neural microenvironment by regulating the passage of molecules and cells into the brain (2). To date, three mechanisms have been proposed for pathogens to cross the BBB: transcellular migration, paracellular migration, and the Trojan horse mechanism (1). Once pathogens have translocated to the brain parenchyma, they proliferate and cause brain inflammation, often with devastating consequences. There are three fundamental questions in the field (Figure 1): (1) How are pathogens arrested in the brain vasculature? (2) How do pathogens migrate into the brain across the BBB? and (3) How do immune cells respond to the brain infection and do they clear the pathogen or cause inflammation in a constrained intracranial compartment that is highly susceptible to cellular dysfunction and increased pressure?

Modern advances in technology have provided opportunities to better understand hostpathogen interactions. Among them, imaging of organs in living animals, using high-resolution intravital microscopy (IVM), represents a major advance in the field. Using this technique, interactions of pathogens with brain endothelial cells, and their transmigration across the BBB can 


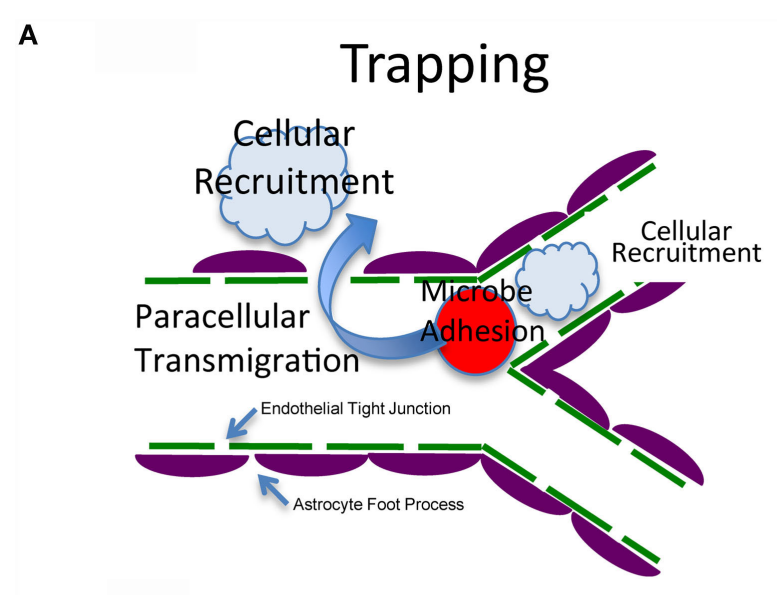

B

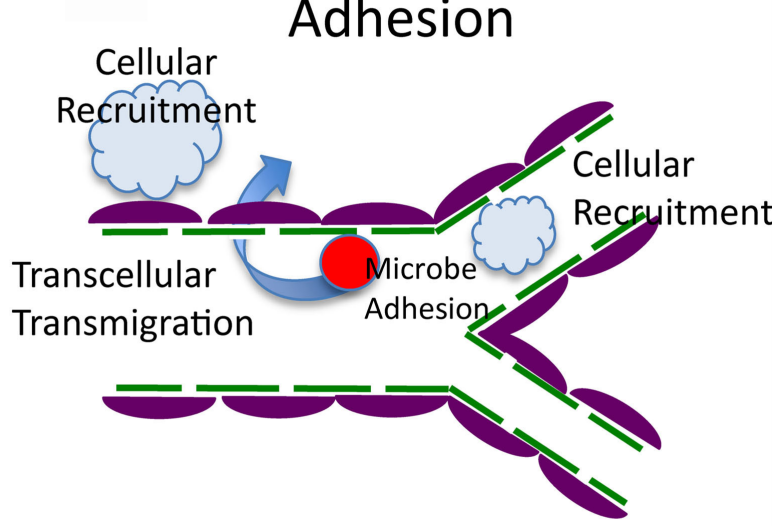

c Trojan Horse

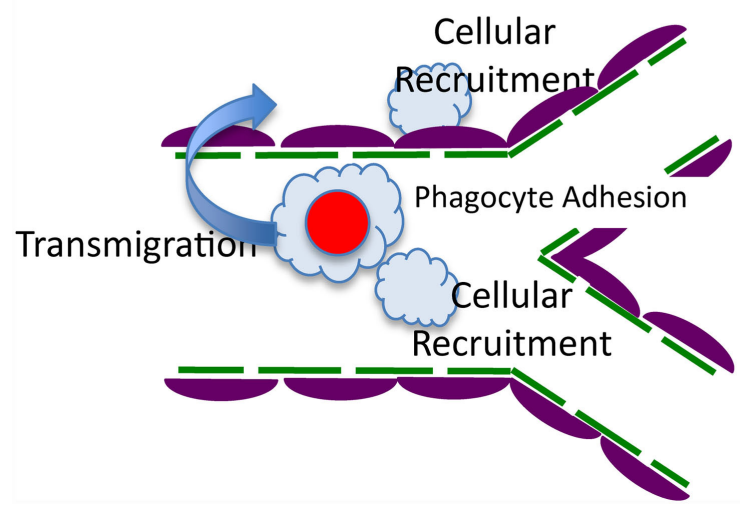

FIGURE 1 | Possible mechanisms of arrest, transmigration, and resultant host response. The BBB is formed by brain endothelial cells, which are connected by tight junctions, and astrocyte foot processes that surround the endothelial cells and maintain the integrity of the BBB $(2,3)$. (A) Fungal cells are trapped by vascular constriction with possible sensing and signaling of both cell types $(4,5)$. This is followed by transmigration that could be by a trans- or paracellular mechanism (paracellular is shown in this panel). Immune and inflammatory cells are recruited to the vascular or extracellular compartment to generate host defense and inflammation.

(B) Fungal cells adhere directly to the endothelium with possible sensing and

(Continued)

\section{FIGURE 1 | Continued}

signaling of both cell types $(6-10)$. This is followed by transmigration that could be by a trans- or paracellular mechanism (transcellular is shown in this panel). Immune and inflammatory cells are recruited to the vascular or extracellular compartment to generate host defense and inflammation. (C) Fungal cells are internalized within a host cell (Trojan Horse) that makes contact with the endothelium, arrests, and generates sensing and signaling of all three cell types $(11,12)$. This is followed by transmigration that could be by a trans- or paracellular mechanism. Immune and inflammatory cells are recruited to the vascular or extracellular compartment to generate host defense and inflammation.

be directly assessed under flow conditions in real time. In addition, the dynamic interactions of leukocytes with pathogens and their behavior in the brain vasculature and parenchyma can be evaluated in living animals. This is of particular importance, because the extravascular migration of pathogens and their interactions with immune cells are transient and highly dynamic, and investigation of these processes by direct observation using IVM provides insights that cannot be obtained using other techniques.

Of the approximately 300 fungal species that have been reported to be pathogenic to humans (13), Cryptococcus neoformans, Candida albicans, Histoplasma capsulatum, Coccidioides immitis, Paracoccidioides brasiliensis, Aspergillus spp., and zygomycetes are among the most common causes of brain or meningeal infections (14-21). In particular, cryptococcal meningoencephalitis is one of the most common infections of the central nervous system and a leading course of HIVassociated mortality globally $(16,18,22)$. In recent years, much progress has been made to understand migration of pathogens and immune responses induced by the invading pathogens in the brain using IVM. This review will discuss recent studies that used IVM to address brain infections by a very limited subset of pathogenic fungi (Table $\mathbf{1}$ ).

\section{INTRAVITAL MICROSCOPY}

Intravital microscopy was first employed by Julius Cohnheim in the nineteenth century to visualize leukocyte trafficking in the tongue and mesentery of a frog (27). In the last decade, significant progress has been made in imaging of live animals due to breakthroughs in microscopy. Wide-field microscopy, multiphoton confocal, spinning disk confocal, and multiphoton resonant scanning confocal microscopy have been used to image fungal infection in the brain. Each imaging system has its advantages or disadvantages depending on whether speed of image acquisition, depth into the tissue, image resolution, photobleaching and phototoxicity, and price are considerations (28-32).

\section{IVM PROCEDURE}

There are two major surgical methods to make the brain vasculature visible under fluorescent microscopy, i.e., a thinned-skull cranial window and an open-skull cranial window (33). Both techniques have advantages and limitations. During imaging through the thinned-skull cranial window, the brain does not 
TABLE 1 | Application of intravital imaging to brain infection by fungi.

\begin{tabular}{|c|c|c|c|}
\hline Fungi & Animals & Nature of the work & Reference \\
\hline C. neoformans & Mouse & $\begin{array}{l}\text { The fungal cell is mechanically } \\
\text { trapped in the brain capillary } \\
\text { and transmigrates to the brain } \\
\text { parenchyma with contributions } \\
\text { from urease }\end{array}$ & Shi et al. (5) \\
\hline C. neoformans & Mouse & $\begin{array}{l}\text { Neutrophils internalize the } \\
\text { intravascular fungal cell that } \\
\text { had been arrested in the brain } \\
\text { microvasculature and return to } \\
\text { the blood stream in a "vacuum- } \\
\text { cleaner" type of behavior }\end{array}$ & $\begin{array}{l}\text { Zhang } \\
\text { et al. (23) }\end{array}$ \\
\hline C. neoformans & Zebrafish & $\begin{array}{l}\text { The fungal cell was observed to } \\
\text { proliferate within macrophages; } \\
\text { capsule size determines early } \\
\text { macrophage control of infection }\end{array}$ & $\begin{array}{l}\text { Bojarczuk } \\
\text { et al. (24) }\end{array}$ \\
\hline C. neoformans & Zebrafish & $\begin{array}{l}\text { The fungal cell can cross the } \\
\text { zebrafish blood-brain barrier, } \\
\text { which is dependent on the FNX1 } \\
\text { virulence gene }\end{array}$ & $\begin{array}{l}\text { Tenor et al. } \\
\text { (25) }\end{array}$ \\
\hline C. albicans & Mouse & $\begin{array}{l}\text { Accumulation of both yeast and } \\
\text { filamentous forms of the fungal } \\
\text { cells were observed in the brain } \\
\text { meninges and parenchyma }\end{array}$ & $\begin{array}{l}\text { Navarathna } \\
\text { et al. (26) }\end{array}$ \\
\hline P. brasiliensis & Mouse & $\begin{array}{l}\text { Enhanced leukocyte recruitment } \\
\text { to the brain following the fungal } \\
\text { infection is associated with CXCL9 }\end{array}$ & $\begin{array}{l}\text { Pedroso } \\
\text { et al. (20) }\end{array}$ \\
\hline
\end{tabular}

need to be superfused with artificial cerebrospinal fluids because the brain tissue is still covered with the skull. It is well suited for observations over long periods of time. However, the skull thickness affects the image quality and achieving optimal and uniform skull thickness requires a high level of surgical proficiency. By contrast, in an open-skull window, a portion of the skull and dura is removed, and the cortical surface is directly exposed to microscopy. Thus, a better quality of images is usually achieved compared with a thinned-skull window. However, it is essential to superfuse the brain with artificial cerebrospinal fluid during the period of observation, and great care must be taken to avoid surgical trauma and hemorrhage (33).

To facilitate intravital imaging, the organisms, brain microvasculature, and leukocytes can be labeled with fluorochromes. For example, we labeled C. neoformans with fluorescent isothiocyanate (FITC) or tetramethylrhodamine isothiocyanate (TRITC) to visualize the arrest and migration of the yeast cell into the brain $(4,5)$. Two colors allow comparison of two different virulence characteristics or wild-type and mutant strains. However, the yeast cell loses the fluorescent label if it proliferates. This disadvantage might be overcome by using fungi expressing green or red fluorescent proteins if sufficient fluorescent intensity can be achieved $(26,34,35)$. To label the microvasculature, rat-anti-mouse PECAM-1 [CD31, a molecule expressed on endothelial cells (36)] can be injected intravenously $(37,38)$. Since the tight junctions of endothelial cells express high PECAM-1, this labeling can be used to study interactions of fungi or leukocytes with endothelial tight junctions (36). Alternatively, the vascular compartment can be illuminated by intravenous injection with fluorochrome-conjugated bovine serum albumin or dextran (39). In addition, transgenic mice that express fluorescent proteins in endothelial cells [for example, Tie-2 green fluorescent protein (GFP) mice (40)] can be used.

An expanding number of tools are becoming available to study the interactions of fungi with immune and inflammatory cells. To determine the trafficking of leukocytes in the brain, mice can be injected intravenously with rhodamine $6 \mathrm{G}$, which is a cellpermeant dye that is sequestered by active mitochondria $(41,42)$. However, to identify the functions of subsets of leukocytes, $\mathrm{mAb}$ or transgenic mice can be used. For example, anti-CD45 can be injected intravenously, which labels all leukocytes. Neutrophils can be labeled in vivo by intravenous injection of anti-Ly6G (23). Alternatively, neutrophils can be visualized in mice expressing enhanced GFP under the control of the endogenous lysozyme promoter (LysM-eGFP) $(39,43)$. To image monocytes, mice can be intravenously injected with fluorochrome-labeled anti-CCR2 (labels proinflammatory monocytes) or anti-CX3CR1 antibody (labels patrolling monocytes) (44). Alternately, CX3CR $1^{\text {gfp/ } /+}$ mice can be used to achieve this goal. In CX3CR1 $1^{\text {fp/ } /+}$ mice, one allele for the gene encoding CX3CR1, the receptor for chemokine CX3CL1, has been replaced with a gene encoding GFP, resulting in GFP expression of all circulating $\mathrm{CD} 11 \mathrm{~b}^{+} \mathrm{F} 4 / 80^{+}$cells. CX3CR $1^{\text {fpp/+ }}$ mice express GFP in monocytes, but not in neutrophils $(45,46)$. With time, many more mouse strains are becoming available that have fluorescent reporters linked to other genes that define different subsets of cells and allow us to study the role of those cells in the pathogenesis of infection.

\section{BRAIN INFECTION WITH C. NEOFORMANS}

Cryptococcus neoformans is an encapsulated budding yeast that causes a life-threatening illness in immunocompromised individuals, especially in AIDS patients. It is estimated that there are one million cases of cryptococcosis per year and 600,000 of these patients will die within 3 months of diagnosis (22). Cryptococcus is found in the environment and enters the body through the respiratory tract. Immunocompetent individuals are usually able to contain C. neoformans in the lung (47). In the case of an immunocompromised host, the yeast cells cannot be successfully contained and disseminate into the brain via the bloodstream, causing meningoencephalitis $(16,47)$.

Hematogenous dissemination of C. neoformans is one of the most critical steps in the development of meningoencephalitis. Prior to transmigration into the brain parenchyma, circulating C. neoformans must be arrested in the brain vasculature. We became interested in a number of questions related to the pathogenesis of cryptococcal meningoencephalitis. (1) Was C. neoformans arrested in the brain vasculature prior to transmigration and did the arrest occur in venules or capillaries? (2) How did C. neoformans behave during arrest? and (3) What was the mechanism(s) underlying the arrest of $C$. neoformans. As arrest of C. neoformans is a transient and dynamic process, we developed an in vivo model system based on IVM to study these questions $(4,5)$. We demonstrated that $C$. neoformans appeared in the mouse brain microvasculature within a few 
seconds after injection into the tail vein. When first seen, C. neoformans was moving with the same velocity as the blood, and no interaction of circulating $C$. neoformans with venular endothelial cells was observed. The number of yeast cells passing through postcapillary venules was greatest immediately after injection and gradually decreased over time. However, even after $18 \mathrm{~h}$, rare yeast cells could still be seen moving in the brain venules. C. neoformans appeared to move at the same speed as the blood and came to a sudden stop in the capillaries of the brain without rolling and tethering to the endothelial surface. Interestingly, the yeast cells were arrested in capillaries that appeared to be of the same or smaller diameter than the organism, often at branch points. Differences in viability, polysaccharide capsule (the major virulence factor), and strain failed to affect the deposition of the yeast cells. In particular, there was no significant difference in the behavior and the arrest of polystyrene microspheres of similar size in the brain capillary bed when compared with $C$. neoformans. These results suggest that $C$. neoformans is mechanically trapped in the brain, which raises novel challenges for therapies to avoid arrest.

Cryptococcus neoformans transmigrates into the brain parenchyma across the BBB after arrest in the brain capillaries. Previous studies, using in vitro techniques, have shown that $C$. neoformans can cross the endothelium of the brain via direct transcytosis $(6,48,49)$. It was further demonstrated that transcytosis is mediated by interactions between CD44 expressed on endothelium and cryptococcal hyaluronic acids $(7,8)$. A secreted fungal metalloprotease (9), an extracellular phospholipase B1 (10), and brain inositol (50) are critically involved in transcytosis of $C$. neoformans. In addition, it was also reported that $C$. neoformans invaded the brain via a "Trojan horse" mechanism with the help of phagocytes $(11,12)$. However, these studies have failed to determine the dynamics of BBB penetration by $C$. neoformans in the brain vasculature in vivo. Using IVM, we have recently characterized the transmigration of C. neoformans in vivo (5). Following arrest in the brain, $C$. neoformans was directly seen to cross the capillary wall of living animals in real time. In contrast to trapping, viability, but not replication, was required for $C$. neoformans to cross the $\mathrm{BBB}$. Urease is critically involved in brain transmigration of the organism. Accordingly, a urease inhibitor could ameliorate infection of the mouse brain by reducing transmigration of $C$. neoformans into the brain, suggesting that a therapeutic strategy aimed at inhibiting this enzyme might be beneficial in cryptococcal meningitis and encephalitis.

Arrest of $C$. neoformans in the brain vasculature led to questions about recognition of the organism by circulating leukocytes. Recently, we addressed this question with the use of IVM (23). Among all subsets of leukocytes in the circulation, neutrophils are the most abundant phagocytes and are usually the first immune cells to be recruited to a site of infection to eliminate pathogens (51). Early work had suggested that human neutrophils kill C. neoformans in vitro via an intracellular $(52,53)$ or extracellular killing mechanism (54). In particular, the capability of human neutrophils to kill the organism was reported to be even greater than that of monocytes $(52,55)$. In vitro, mouse neutrophils appear to move toward $C$. neoformans and then rapidly internalize the yeast (56). Complement C5a-C5aR signaling was essential for phagocytosis of $C$. neoformans by neutrophils by guiding their migration to neutrophils and enhancing surface expression of CD11b (56). Furthermore, the p38 MAPK pathway, but not the Erk pathway, was critically involved in C5a-C5aR-mediated chemotaxis of neutrophils during their killing of C. neoformans (56). These in vitro observations encouraged us to address how neutrophils dynamically interact with $C$. neoformans which were arrested in the brain vasculature (23). With the use of IVM, we demonstrated that neutrophils crawled to the yeast cells that had been arrested in the brain microvasculature. Interestingly, crawling neutrophils recognized and interacted with the yeast, resulting in internalization of C. neoformans. During the interactions of neutrophils with the yeast, morphologic alterations of neutrophils, including deploying pseudopodia, were observed. Internalization of C. neoformans by neutrophils in the brain vasculature could be completed within a few minutes. Following ingestion of C. neoformans, neutrophils were seen to crawl again along the vessel wall and eventually to be released into the blood flow, resulting in a direct removal of the arrested $C$. neoformans from the brain vasculature. Depletion of neutrophils enhanced brain fungal burden (23), while enhancing the recruitment of neutrophils improved intravascular clearance of $C$. neoformans in the brain (57). Further studies demonstrated that C. neoformans infection led to enhanced expression of the adhesion molecule, Mac-1, on neutrophils, and ICAM-1 on brain endothelial cells. Complement C3 was critically involved in the recognition of $C$. neoformans by neutrophils and subsequent clearance of the organism from the brain (23). These results revealed that neutrophils are able to remove C. neoformans that had been arrested in the brain microvasculature in a "vacuumcleaner" type of behavior. Given that neutrophils are usually considered to kill microorganisms at the infection site, the finding of the direct removal of $C$. neoformans by neutrophils from its arrested site may represent a novel mechanism of host defense in the brain (23). In this respect, neutrophils have been recently shown to "sweep up" bacteria arrested on the walls of an infected body cavity or blood vessel, but not fluid-borne bacteria in a zebrafish model (58).

Recently, a live-imaging model based on zebrafish larvae has been established to study the interactions of $C$. neoformans with innate immune cells and its migration to the brain $(24,25)$. The zebrafish $C$. neoformans platform provides a visually and genetically accessible vertebrate model system for infection of C. neoformans. It was shown that zebrafish macrophages rapidly phagocytosed the majority of $C$. neoformans cells following injection of the yeast via the caudal vein (25). Depletion of macrophages significantly enhanced the fungal burden in zebrafish, demonstrating that macrophages are essential to protect zebrafish from disease progression $(24,25)$. However, macrophages preferentially ingested $C$. neoformans with smaller polysaccharide capsules, and since the capsule size greatly increased over $24 \mathrm{~h}$ of infection, this markedly limited further phagocytosis (24). In addition, proliferation of C. neoformans within macrophages and non-lytic exocytosis of the yeast from macrophages were observed in zebrafish (24). Live 
imaging demonstrated that $C$. neoformans is able to penetrate the zebrafish brain. There was a positive correlation between the burden of organisms in cranial vessels versus invasion into the brain parenchyma (25). The C. neoformans fnx $1 \Delta$ mutant, which is deficient in a multidrug resistance-like protein, was shown to have a deficiency in transmigration across the mouse BBB and reduced microvascular entrapment and transcytosis across immortalized human brain capillary endothelial cells in vitro (59). Interestingly, the fnx $1 \Delta$ mutant also demonstrated defective invasion of brain parenchyma of zebrafish (25). Using IVM, these studies are just beginning to enhance our understanding of the spacial and temporal aspects and the role of different cell types in pathogenesis and host defense to C. neoformans.

\section{BRAIN INFECTION WITH C. ALBICANS}

Candida albicans is a commensal organism and a common constituent of the normal mucosal flora. As the most common fungal pathogen of humans, overgrowth causes thrush. However, translocation of the yeast cells from the mucosal surface into the systemic circulation causes potentially life-threatening disease, particularly in post-surgical and critically ill patients, which is associated with approximately $35 \%$ death rate $(60,61)$. During this disease, the bloodborne organisms can spread to virtually all organs of the body. Although the kidney is the primary target of this organism during disseminated candidiasis, brain infection is found in approximately one-half of patients with systemic candidiasis at autopsy (62-64). In addition, C. albicans has also been reported to cause meningoencephalitis without systemic infection in healthy individuals (65).

To invade the brain parenchyma, circulating C. albicans cells must adhere and cross the BBB. Early work had shown that C. albicans is able to penetrate a monolayer of human brain endothelial cells cultured in vitro via a transcellular pathway (66). It was later demonstrated that C. albicans invasion of brain endothelial cells is mediated by the fungal invasins Als3 and Ssa1 (67). Als3 binds to the gp96 heat shock protein, a unique receptor that is expressed specifically on brain endothelium, promoting endothelial transcytosis by the fungus (67).

Recently, Navarathna et al. studied brain infection by C. albicans in a mouse model using IVM (26). They observed sporadic entry of C. albicans into the brain parenchyma as early as 30 min after intravenous inoculation. In this model, the authors did not observeleak of gadolinium diethylenetriaminepentaacetic acid (Gd-DTPA) into the brain 30 min after intravenous administration as examined by MRI, suggesting that brain invasion by $C$. albicans initially occurs without gross disruption of the $\mathrm{BBB}$. However, IVM performed 3 days post-infection revealed significant accumulation of both yeast and filamentous forms of C. albicans in the meninges and parenchyma. At that time, leak of Gd-DTPA was observed, indicating damage of the BBB. The brain became heavily inflamed at sites of $C$. albicans invasion. Thus, it is conceivable that permeability of the BBB was caused by leukocyte infiltration. In addition, Candida filament elongation was observed in the brain. Interestingly, most of the yeast cells outside of the vasculature showed highly dynamic movement that could be explained by the movement of phagocytosed organisms within motile phagocytic cells. By contrast, hyphal cells showed only slow invasion based on hyphal extension.

\section{BRAIN INFECTION WITH P. BRASILIENSIS}

Paracoccidioides brasiliensis is an etiologic agent of paracoccidioidomycosis, an important systemic mycosis in Latin America, with the greatest number of patients in Brazil, Venezuela, and Argentina (68). The infection is usually acquired by the respiratory system probably by inhalation of airborne conidia of $P$. brasiliensis $(69,70)$. Following infection, the conidia transform into yeast in the lungs. $P$. brasiliensis can cause disease in immunocompetent hosts, although immunosuppression increases the severity of infection. The yeast cells can be disseminated from the infected lung into other organs such as adrenal glands and brain $(70,71)$. In the last decade, brain infection has been reported more commonly, affecting approximately $12.5 \%$ of cases (70). However, it is unknown how the fungus arrests and migrates into the brain parenchyma across the BBB.

Recently, Pedroso et al. used IVM to examine trafficking of leukocytes in the brain in a murine model of neuroparacoccidioidomycosis (20). Following infection with P. brasiliensis by the intracranial route, mice showed clinical signs of progressive infection starting on day 7 post-inoculation. IVM of the brain pial microvasculature revealed a significant increase in leukocyte rolling 2 and 4 weeks post-infection and in adhesion 1, 2, and 4 weeks post-infection. The enhanced recruitment of leukocytes was associated with a significant increase in the brain concentration of chemokines, particularly CXCL9, suggesting a role for these molecules in the inflammatory and immune response against the fungi. The lesions were not restricted to the site of inoculation and disseminated to other sites of the brain including the cerebellum. Neutrophils and macrophages were increased in the brain as determined by the myeloperoxidase and $N$-acetyl-bD-glucosaminidase activity in the brain tissues.

\section{CONCLUDING REMARKS}

Fungal meningoencephalitis is a grave illness associated with high mortality, even with the best available antifungal treatment. Understanding the mechanisms involved in arrest and invasion of the brain by fungi and the interactions with immune cells is fundamental to our knowledge of the pathogenesis of the disease. With the use of IVM, brain infections by fungi, including C. neoformans $(5,23)$, C. albicans (26), and P. brasiliensis (20), have been recently investigated in real time. In particular, we have shown that $C$. neoformans is mechanically trapped in the brain vasculature (5). IVM may provide a powerful tool to determine whether in vitro findings implicating interactions between CD44 and hyaluronic acid $(7,8)$, or adherence of phagocytosed cells (Trojan Horse) also occur $(11,12)$ through the use of transgenic mice. Although neutrophils are able to recognize and remove the arrested C. neoformans from the brain vasculature (23), organisms were seen to cross the vessel wall with contribution of cryptococcal urease (5). IVM may provide a powerful tool to investigate the role of metalloprotease (9), and phospholipase (10) in brain invasion via transcytosis through the use of deletion 
mutants. The role of host immune cells in brain injury has been implicated in studies using C. albicans, and chemokines have been implicated by studies of $P$. brasiliensis $(20,26)$. In summary, recent advances in microscopy and the availability of antibody labeling in vivo, transgenic reporter mice and mice with targeted gene disruptions provide a powerful tool to examine fungal brain infections under more relevant physiologic conditions. It is expected that exciting findings regarding spacial and temporal aspects of pathogenic and immune mechanisms in fungal brain infections will be obtained with the application of IVM in the future, and that these insights may lead to new therapies.

\section{REFERENCES}

1. Kim KS. Mechanisms of microbial traversal of the blood-brain barrier. Nat Rev Microbiol (2008) 6(8):625-34. doi:10.1038/nrmicro1952

2. Owens T, Bechmann I, Engelhardt B. Perivascular spaces and the two steps to neuroinflammation. J Neuropathol Exp Neurol (2008) 67(12):1113-21. doi:10.1097/NEN.0b013e31818f9ca8

3. Engelhardt B, Sorokin L. The blood-brain and the blood-cerebrospinal fluid barriers: function and dysfunction. Semin Immunopathol (2009) 31(4):497511. doi:10.1007/s00281-009-0177-0

4. Shi M, Colarusso P, Mody CH. Real-time in vivo imaging of fungal migration to the central nervous system. Cell Microbiol (2012) 14(12):1819-27. doi: $10.1111 / \mathrm{cmi} .12027$

5. Shi M, Li SS, Zheng C, Jones GJ, Kim KS, Zhou H, et al. Real-time imaging of trapping and urease-dependent transmigration of Cryptococcus neoformans in mouse brain. J Clin Invest (2010) 120(5):1683-93. doi:10.1172/JCI41963

6. Chang YC, Stins MF, McCaffery MJ, Miller GF, Pare DR, Dam T, et al. Cryptococcal yeast cells invade the central nervous system via transcellular penetration of the blood-brain barrier. Infect Immun (2004) 72(9):4985-95. doi:10.1128/IAI.72.9.4985-4995.2004

7. Jong A, Wu CH, Gonzales-Gomez I, Kwon-Chung KJ, Chang YC, Tseng HK, et al. Hyaluronic acid receptor CD44 deficiency is associated with decreased Cryptococcus neoformans brain infection. J Biol Chem (2012) 287(19): 15298-306. doi:10.1074/jbc.M112.353375

8. Jong A, Wu CH, Shackleford GM, Kwon-Chung KJ, Chang YC, Chen HM, et al. Involvement of human CD44 during Cryptococcus neoformans infection of brain microvascular endothelial cells. Cell Microbiol (2008) 10(6):1313-26. doi:10.1111/j.1462-5822.2008.01128.x

9. $\mathrm{Vu}$ K, Tham R, Uhrig JP, Thompson GR III, Na Pombejra S, Jamklang M, et al. Invasion of the central nervous system by Cryptococcus neoformans requires a secreted fungal metalloprotease. MBio (2014) 5(3):e1101-14. doi:10.1128/ mBio.01101-14

10. Maruvada R, Zhu L, Pearce D, Zheng Y, Perfect J, Kwon-Chung KJ, et al. Cryptococcus neoformans phospholipase B1 activates host cell Racl for traversal across the blood-brain barrier. Cell Microbiol (2012) 14(10):1544-53. doi:10.1111/j.1462-5822.2012.01819.x

11. Charlier C, Nielsen K, Daou S, Brigitte M, Chretien F, Dromer F. Evidence of a role for monocytes in dissemination and brain invasion by Cryptococcus neoformans. Infect Immun (2009) 77(1):120-7. doi:10.1128/IAI.01065-08

12. Sorrell TC, Juillard PG, Djordjevic JT, Kaufman-Francis K, Dietmann A, Milonig A, et al. Cryptococcal transmigration across a model brain blood-barrier: evidence of the Trojan horse mechanism and differences between Cryptococcus neoformans var. grubii strain $\mathrm{H} 99$ and Cryptococcus gattii strain R265. Microbes Infect (2016) 18(1):57-67. doi:10.1016/j.micinf.2015.08.017

13. Bryan AM, Del Poeta M, Luberto C. Sphingolipids as regulators of the phagocytic response to fungal infections. Mediators Inflamm (2015) 2015:640540. doi:10.1155/2015/640540

14. Bariola JR, Perry P, Pappas PG, Proia L, Shealey W, Wright PW, et al. Blastomycosis of the central nervous system: a multicenter review of diagnosis and treatment in the modern era. Clin Infect Dis (2010) 50(6):797-804. doi:10.1086/650579

15. Chakrabarti A. Epidemiology of central nervous system mycoses. Neurol India (2007) 55(3):191-7. doi:10.4103/0028-3886.35679

\section{AUTHOR CONTRIBUTIONS}

All authors listed have made substantial, direct, and intellectual contribution to the work and approved it for publication.

\section{FUNDING}

This work was supported by U.S. National Institutes of Health National Institute of Allergy and Infectious Diseases Grant AI115086A (to MS) and Canadian Institute for Health Research Grant 247301 (to CM).

16. Gottfredsson M, Perfect JR. Fungal meningitis. Semin Neurol (2000) 20(3):307-22. doi:10.1055/s-2000-9394

17. Kleinschmidt-DeMasters BK. Central nervous system aspergillosis: a 20-year retrospective series. Hum Pathol (2002) 33(1):116-24. doi:10.1053/hupa. 2002.30186

18. Liu TB, Perlin DS, Xue C. Molecular mechanisms of cryptococcal meningitis. Virulence (2012) 3(2):173-81. doi:10.4161/viru.18685

19. Murthy JM. Fungal infections of the central nervous system: the clinical syndromes. Neurol India (2007) 55(3):221-5. doi:10.4103/0028-3886.35682

20. Pedroso VS, Vilela MC, Santos PC, Cisalpino PS, Rachid MA, Teixeira AL. Traffic of leukocytes and cytokine up-regulation in the central nervous system in a murine model of neuroparacoccidioidomycosis. Mycopathologia (2013) 176(3-4):191-9. doi:10.1007/s11046-013-9679-3

21. Scully EP, Baden LR, Katz JT. Fungal brain infections. Curr Opin Neurol (2008) 21(3):347-52. doi:10.1097/WCO.0b013e3282fee95b

22. Park BJ, Wannemuehler KA, Marston BJ, Govender N, Pappas PG, Chiller TM. Estimation of the current global burden of cryptococcal meningitis among persons living with HIV/AIDS. AIDS (2009) 23(4):525-30. doi:10.1097/ QAD.0b013e328322ffac

23. Zhang M, Sun D, Liu G, Wu H, Zhou H, Shi M. Real-time in vivo imaging reveals the ability of neutrophils to remove Cryptococcus neoformans directly from the brain vasculature. J Leukoc Biol (2016) 99(3):467-73. doi:10.1189/ jlb.4AB0715-281R

24. Bojarczuk A, Miller KA, Hotham R, Lewis A, Ogryzko NV, Kamuyango AA, et al. Cryptococcus neoformans intracellular proliferation and capsule size determines early macrophage control of infection. Sci Rep (2016) 6:21489. doi:10.1038/srep21489

25. Tenor JL, Oehlers SH, Yang JL, Tobin DM, Perfect JR. Live imaging of host-parasite interactions in a zebrafish infection model reveals cryptococcal determinants of virulence and central nervous system invasion. MBio (2015) 6(5):e1425-1415. doi:10.1128/mBio.01425-15

26. Navarathna DH, Munasinghe J, Lizak MJ, Nayak D, McGavern DB, Roberts DD. MRI confirms loss of blood-brain barrier integrity in a mouse model of disseminated candidiasis. NMR Biomed (2013) 26(9):1125-34. doi:10.1002/ nbm. 2926

27. Dutrochet H. Recherches Anatomiques et Physiologiques sur la Structure Intime des Animaux et des Vegetaux, et sur Leur Motilite. Paris: Bailliere et fils (1824)

28. Helmchen F, Denk W. Deep tissue two-photon microscopy. Nat Methods (2005) 2(12):932-40. doi:10.1038/nmeth818

29. Graf R, Rietdorf J, Zimmermann T. Live cell spinning disk microscopy. Adv Biochem Eng Biotechnol (2005) 95:57-75. doi:10.1007/b102210

30. Murray JM. Methods for imaging thick specimens: confocal microscopy, deconvolution, and structured illumination. Cold Spring Harb Protoc (2011) 2011(12):1399-437. doi:10.1101/pdb.top066936

31. Conchello JA, Lichtman JW. Optical sectioning microscopy. Nat Methods (2005) 2(12):920-31. doi:10.1038/nmeth815

32. Ustione A, Piston DW. A simple introduction to multiphoton microscopy. J Microsc (2011) 243(3):221-6. doi:10.1111/j.1365-2818.2011.03532.x

33. Yang G, Pan F, Parkhurst CN, Grutzendler J, Gan WB. Thinned-skull cranial window technique for long-term imaging of the cortex in live mice. Nat Protoc (2010) 5(2):201-8. doi:10.1038/nprot.2009.222

34. Lionakis MS, Swamydas M, Fischer BG, Plantinga TS, Johnson MD, Jaeger M, et al. CX3CR1-dependent renal macrophage survival promotes Candida 
control and host survival. J Clin Invest (2013) 123(12):5035-51. doi:10.1172/ JCI71307

35. Voelz K, Johnston SA, Rutherford JC, May RC. Automated analysis of cryptococcal macrophage parasitism using GFP-tagged cryptococci. PLoS One (2010) 5(12):e15968. doi:10.1371/journal.pone.0015968

36. Albelda SM, Muller WA, Buck CA, Newman PJ. Molecular and cellular properties of PECAM-1 (endoCAM/CD31): a novel vascular cell-cell adhesion molecule. J Cell Biol (1991) 114(5):1059-68. doi:10.1083/jcb.114.5.1059

37. Moriarty TJ, Norman MU, Colarusso P, Bankhead T, Kubes P, Chaconas G. Real-time high resolution 3D imaging of the Lyme disease spirochete adhering to and escaping from the vasculature of a living host. PLoS Pathog (2008) 4(6):e1000090. doi:10.1371/journal.ppat.1000090

38. Phillipson M, Heit B, Colarusso P, Liu L, Ballantyne CM, Kubes P. Intraluminal crawling of neutrophils to emigration sites: a molecularly distinct process from adhesion in the recruitment cascade. J Exp Med (2006) 203(12):2569-75. doi:10.1084/jem.20060925

39. McDonald B, Pittman K, Menezes GB, Hirota SA, Slaba I, Waterhouse CC, et al. Intravascular danger signals guide neutrophils to sites of sterile inflammation. Science (2010) 330(6002):362-6. doi:10.1126/science.1195491

40. Motoike T, Loughna S, Perens E, Roman BL, Liao W, Chau TC, et al. Universal GFP reporter for the study of vascular development. Genesis (2000) 28(2):7581. doi:10.1002/1526-968X(200010)28:2<75::AID-GENE50>3.0.CO;2-S

41. Zhou H, Andonegui G, Wong CH, Kubes P. Role of endothelial TLR4 for neutrophil recruitment into central nervous system microvessels in systemic inflammation. J Immunol (2009) 183(8):5244-50. doi:10.4049/ jimmunol.0901309

42. Zhou H, Lapointe BM, Clark SR, Zbytnuik L, Kubes P. A requirement for microglial TLR4 in leukocyte recruitment into brain in response to lipopolysaccharide. JImmunol (2006) 177(11):8103-10. doi:10.4049/ jimmunol.177.11.8103

43. Faust N, Varas F, Kelly LM, Heck S, Graf T. Insertion of enhanced green fluorescent protein into the lysozyme gene creates mice with green fluorescent granulocytes and macrophages. Blood (2000) 96(2):719-26.

44. Auffray C, Fogg D, Garfa M, Elain G, Join-Lambert O, Kayal S, et al. Monitoring of blood vessels and tissues by a population of monocytes with patrolling behavior. Science (2007) 317(5838):666-70. doi:10.1126/science.1142883

45. Geissmann F, Jung S, Littman DR. Blood monocytes consist of two principal subsets with distinct migratory properties. Immunity (2003) 19(1):71-82. doi:10.1016/S1074-7613(03)00174-2

46. Jung S, Aliberti J, Graemmel P, Sunshine MJ, Kreutzberg GW, Sher A, et al. Analysis of fractalkine receptor CX(3)CR1 function by targeted deletion and green fluorescent protein reporter gene insertion. Mol Cell Biol (2000) 20(11):4106-14. doi:10.1128/MCB.20.11.4106-4114.2000

47. Kwon-Chung KJ, Sorrell TC, Dromer F, Fung E, Levitz SM. Cryptococcosis: clinical and biological aspects. Med Mycol (2000) 38(Suppl 1):205-13. doi:10.1080/mmy.38.s1.205.213

48. Sabiiti W, May RC. Capsule independent uptake of the fungal pathogen Cryptococcus neoformans into brain microvascular endothelial cells. PLoS One (2012) 7(4):e35455. doi:10.1371/journal.pone.0035455

49. Vu K, Weksler B, Romero I, Couraud PO, Gelli A. Immortalized human brain endothelial cell line HCMEC/D3 as a model of the blood-brain barrier facilitates in vitro studies of central nervous system infection by Cryptococcus neoformans. Eukaryot Cell (2009) 8(11):1803-7. doi:10.1128/EC.00240-09

50. Liu TB, Kim JC, Wang Y, Toffaletti DL, Eugenin E, Perfect JR, et al. Brain inositol is a novel stimulator for promoting Cryptococcus penetration of the blood-brain barrier. PLoS Pathog (2013) 9(4):e1003247. doi:10.1371/journal. ppat. 1003247

51. Kolaczkowska E, Kubes P. Neutrophil recruitment and function in health and inflammation. Nat Rev Immunol (2013) 13(3):159-75. doi:10.1038/nri3399

52. Diamond RD, Root RK, Bennett JE. Factors influencing killing of Cryptococcus neoformans by human leukocytes in vitro. J Infect Dis (1972) 125(4):367-76. doi:10.1093/infdis/125.4.367

53. Kozel TR, Highison B, Stratton CJ. Localization on encapsulated Cryptococcus neoformans of serum components opsonic for phagocytosis by macrophages and neutrophils. Infect Immun (1984) 43(2):574-9.

54. Qureshi A, Subathra M, Grey A, Schey K, Del Poeta M, Luberto C. Role of sphingomyelin synthase in controlling the antimicrobial activity of neutrophils against Cryptococcus neoformans. PLoS One (2010) 5(12):e15587. doi:10.1371/ journal.pone.0015587
55. Miller MF, Mitchell TG. Killing of Cryptococcus neoformans strains by human neutrophils and monocytes. Infect Immun (1991) 59(1):24-8.

56. Sun D, Zhang M, Liu G, Wu H, Zhu X, Zhou H, et al. Real-time imaging of interactions of neutrophils with Cryptococcus neoformans demonstrates a crucial role of complement C5a-C5aR signaling. Infect Immun (2015) 84(1):216-29. doi:10.1128/IAI.01197-15

57. Sun D, Zhang M, Liu L, Wu H, Li C, Zhou H, et al. Intravascular clearance of disseminating Cryptococcus neoformans in the brain can be improved by enhancing neutrophil recruitment in mice. Eur J Immunol (2016) 46(7):170414. doi:10.1002/eji.201546239

58. Colucci-Guyon E, Tinevez JY, Renshaw SA, Herbomel P. Strategies of professional phagocytes in vivo: unlike macrophages, neutrophils engulf only surface-associated microbes. J Cell Sci (2011) 124(Pt 18):3053-9. doi:10.1242/ jcs. 082792

59. Tseng HK, Liu CP, Price MS, Jong AY, Chang JC, Toffaletti DL, et al. Identification of genes from the fungal pathogen Cryptococcus neoformans related to transmigration into the central nervous system. PLoS One (2012) 7(9):e45083. doi:10.1371/journal.pone.0045083

60. Horn DL, Neofytos D, Anaissie EJ, Fishman JA, Steinbach WJ, Olyaei AJ, et al. Epidemiology and outcomes of candidemia in 2019 patients: data from the prospective antifungal therapy alliance registry. Clin Infect Dis (2009) 48(12):1695-703. doi:10.1086/599039

61. Wisplinghoff H, Bischoff T, Tallent SM, Seifert H, Wenzel RP, Edmond MB. Nosocomial bloodstream infections in US hospitals: analysis of 24,179 cases from a prospective nationwide surveillance study. Clin Infect Dis (2004) 39(3):309-17. doi:10.1086/421946

62. Parker JC Jr, McCloskey JJ, Lee RS. Human cerebral candidosis - a postmortem evaluation of 19 patients. Hum Pathol (1981) 12(1):23-8. doi:10.1016/ S0046-8177(81)80238-9

63. Pendlebury WW, Perl DP, Munoz DG. Multiple microabscesses in the central nervous system: a clinicopathologic study. J Neuropathol Exp Neurol (1989) 48(3):290-300. doi:10.1097/00005072-198905000-00006

64. Sanchez-Portocarrero J, Perez-Cecilia E, Corral O, Romero-Vivas J, Picazo JJ. The central nervous system and infection by Candida species. Diagn Microbiol Infect Dis (2000) 37(3):169-79. doi:10.1016/S0732-8893(00)00140-1

65. Borha A, Parienti JJ, Emery E, Coskun O, Khouri S, Derlon JM. [Candida albicans cerebral granuloma in an immunocompetent patient. A case report]. Neurochirurgie (2009) 55(1):57-62. doi:10.1016/j.neuchi.2008.06.001

66. Jong AY, Stins MF, Huang SH, Chen SH, Kim KS. Traversal of Candida albicans across human blood-brain barrier in vitro. Infect Immun (2001) 69(7):4536-44. doi:10.1128/IAI.69.7.4536-4544.2001

67. Liu Y, Mittal R, Solis NV, Prasadarao NV, Filler SG. Mechanisms of Candida albicans trafficking to the brain. PLoS Pathog (2011) 7(10):e1002305. doi:10.1371/journal.ppat.1002305

68. de Oliveira HC, Assato PA, Marcos CM, Scorzoni L, de Paula ESAC, Da Silva Jde F, et al. Paracoccidioides-host interaction: an overview on recent advances in the paracoccidioidomycosis. Front Microbiol (2015) 6:1319. doi:10.3389/ fmicb.2015.01319

69. McEwen JG, Bedoya V, Patino MM, Salazar ME, Restrepo A. Experimental murine paracoccidiodomycosis induced by the inhalation of conidia. J Med Vet Mycol (1987) 25(3):165-75. doi:10.1080/02681218780000231

70. Pedroso VS, Vilela Mde C, Pedroso ER, Teixeira AL. [Paracoccidioidomycosis compromising the central nervous system: a systematic review of the literature]. Rev Soc Bras Med Trop (2009) 42(6):691-7. doi:10.1590/S003786822009000600016

71. Paniago AM, de Oliveira PA, Aguiar ES, Aguiar JI, da Cunha RV, Leme LM, et al. Neuroparacoccidioidomycosis: analysis of 13 cases observed in an endemic area in Brazil. Trans R Soc Trop Med Hyg (2007) 101(4):414-20. doi:10.1016/j.trstmh.2006.07.006

Conflict of Interest Statement: The authors declare that the research was conducted in the absence of any commercial or financial relationships that could be construed as a potential conflict of interest.

Copyright $\odot 2016$ Shi and Mody. This is an open-access article distributed under the terms of the Creative Commons Attribution License (CC BY). The use, distribution or reproduction in other forums is permitted, provided the original author(s) or licensor are credited and that the original publication in this journal is cited, in accordance with accepted academic practice. No use, distribution or reproduction is permitted which does not comply with these terms. 


\title{
Role of tumor necrosis factor superfamily in neuroinflammation and autoimmunity
}

\author{
Sandip Sonar and Girdhari Lal ${ }^{*}$ \\ National Centre for Cell Science, Pune, India
}

Tumor necrosis factor superfamily (TNFSF) molecules play an important role in the activation, proliferation, differentiation, and migration of immune cells into the central nervous system (CNS). Several TNF superfamily molecules are known to control alloimmunity, autoimmunity, and immunity. Development of transgenic and gene knockout animals, and monoclonal antibodies against TNFSF molecules have increased our understanding of individual receptor-ligand interactions, and their intracellular signaling during homeostasis and neuroinflammation. A strong clinical association has been observed between TNFSF members and CNS autoimmunity such as multiple sclerosis and also in its animal model experimental autoimmune encephalomyelitis. Therefore, they are promising targets for

OPEN ACCESS

Edited by:

Wolfgang Weninger,

Centenary Institute, Australia

Reviewed by:

Irun R. Cohen,

Weizmann Institute of Science, Israel

Amanda Katherine Huber,

University of Michigan, USA

*Correspondence: Girdhari Lal,

National Centre for Cell Science, NCCS Complex, Ganeshkhind, Pune, Maharashtra 411007, India glal@nccs.res.in

Specialty section: This article was submitted to Multiple Sclerosis and Neuroimmunology, a section of the journal Frontiers in Immunology

Received: 31 March 2015 Accepted: 05 July 2015

Published: 20 July 2015

Citation:

Sonar S and Lal G (2015) Role of tumor necrosis factor superfamily in neuroinflammation and autoimmunity.

Front. Immunol. 6:364.

doi: 10.3389/fimmu.2015.00364 alternative therapeutic options to control autoimmunity. Although, TNFSF ligands are widely distributed and have diverse functions, we have restricted the discussions in this review to TNFSF receptor-ligand interactions and their role in the pathogenesis of neuroinflammation and CNS autoimmunity.

Keywords: autoimmunity, blood-brain barrier, multiple sclerosis, tumor necrosis factor, neuroinflammation

\section{Introduction}

$\mathrm{CD} 4{ }^{+} \mathrm{T}$ cells are one of the key adaptive immune cells that play an important role in several autoimmune diseases like multiple sclerosis (MS), experimental autoimmune encephalomyelitis (EAE), inflammatory bowel disease (IBD), and collagen-induced arthritis (CIA). Interactions between tumor necrosis factor superfamily (TNFSF) ligands and TNF receptor superfamily (TNFRSF) receptors provide the costimulatory signals that control the survival, proliferation, differentiation, and effector function of immune cells. Therefore, signaling from these ligand-receptor pairs effectively

\footnotetext{
Abbreviations: APC, antigen-presenting cells; APRIL, a proliferation-inducing ligand; BAFF, B-cell activating factor; BBB, blood-brain barrier; BCMA, B-cell maturation factor; CNS, central nervous system; CTLA-4, cytotoxic T-lymphocyteassociated protein-4; DR, death domain containing receptor; EAE, experimental autoimmune encephalomyelitis; GA, glatiramer acetate; GITR, glucocorticoid-induced TNFR family-related gene; GITRL, glucocorticoid-induced TNFR familyrelated gene ligand; gld, generalized lymphoproliferative disease; GM-CSF, granulocyte-macrophage colony-stimulating factor; HEVM, herpes virus entry mediator; ICAM-1, intercellular adhesion molecule-1; LIGHT, homologous to lymphotoxins, exhibits inducible expression, and competes with HSV glycoprotein D for herpesvirus entry mediator (HVEM), a receptor expressed by T lymphocytes; lpr, lymphoproliferative; LT $\alpha$, lymphotoxin alpha; LT $\beta R$, lymphotoxin receptor beta; MMP, matrix metallopeptidase; MOG, myelin oligodendrocyte glycoprotein; MS, multiple sclerosis; RANK, receptor activator of NF- $\kappa B$; RANKL, receptor activator of NF- $\kappa B$ ligand; TACE, TNF alpha converting enzyme; TACI, transmembrane activator, calcium modulator, and cyclophilin ligand interactor; TNF, tumor necrosis factor; TNFR, TNF receptor; TNFRSF, TNF receptor superfamily; TNFSF, TNF superfamily; TRAF, TNF receptor-associated factor; TRAIL, TNF-related apoptosisinducing ligand; TRAILR, TNF-related apoptosis-inducing ligand receptor; Treg, regulatory CD4 T cells; TWEAK, TNF-related weak inducer of apoptosis; VCAM-1, vascular cell adhesion molecule-1; VEGI, vascular endothelial cell-growth inhibitor.
} 
helps in maintaining immune cell homeostasis and in regulating the pathology of autoimmune diseases (1-4).

About 19 TNFSF ligands have been identified, which include TNF- $\alpha$, TNF- $\beta$ [also known as lymphotoxin alpha (LT $\alpha)$ ], lymphotoxin- $\beta$ (LT- $\beta$ ), CD27L, CD30L, CD40L, FasL, 4-1BBL, OX40L, TNF-related apoptosis-inducing ligand (TRAIL), LIGHT (homologous to lymphotoxins, exhibits inducible expression, and competes with HSV glycoprotein D for herpesvirus entry mediator (HVEM), a receptor expressed by $\mathrm{T}$ lymphocytes), receptor activator of NF- $\kappa \mathrm{B}$ ligand (RANKL), TNF-related weak inducer of apoptosis (TWEAK), a proliferation-inducing ligand (APRIL), B-cell activating factor (BAFF), vascular endothelial cell-growth inhibitor (VEGI), ectodysplasin A (EDA-A1, EDAA2), and glucocorticoid-induced TNFR family-related gene ligand (GITRL) (Figure 1). While expressions of TNFSF ligands are induced largely on professional antigen-presenting cells (APCs; dendritic cells, B cells, macrophages), their expression is also reported on T cells, NK cells, mast cells, eosinophils, basophils, endothelial cells, thymic epithelial cells, and smooth muscle cells (5).

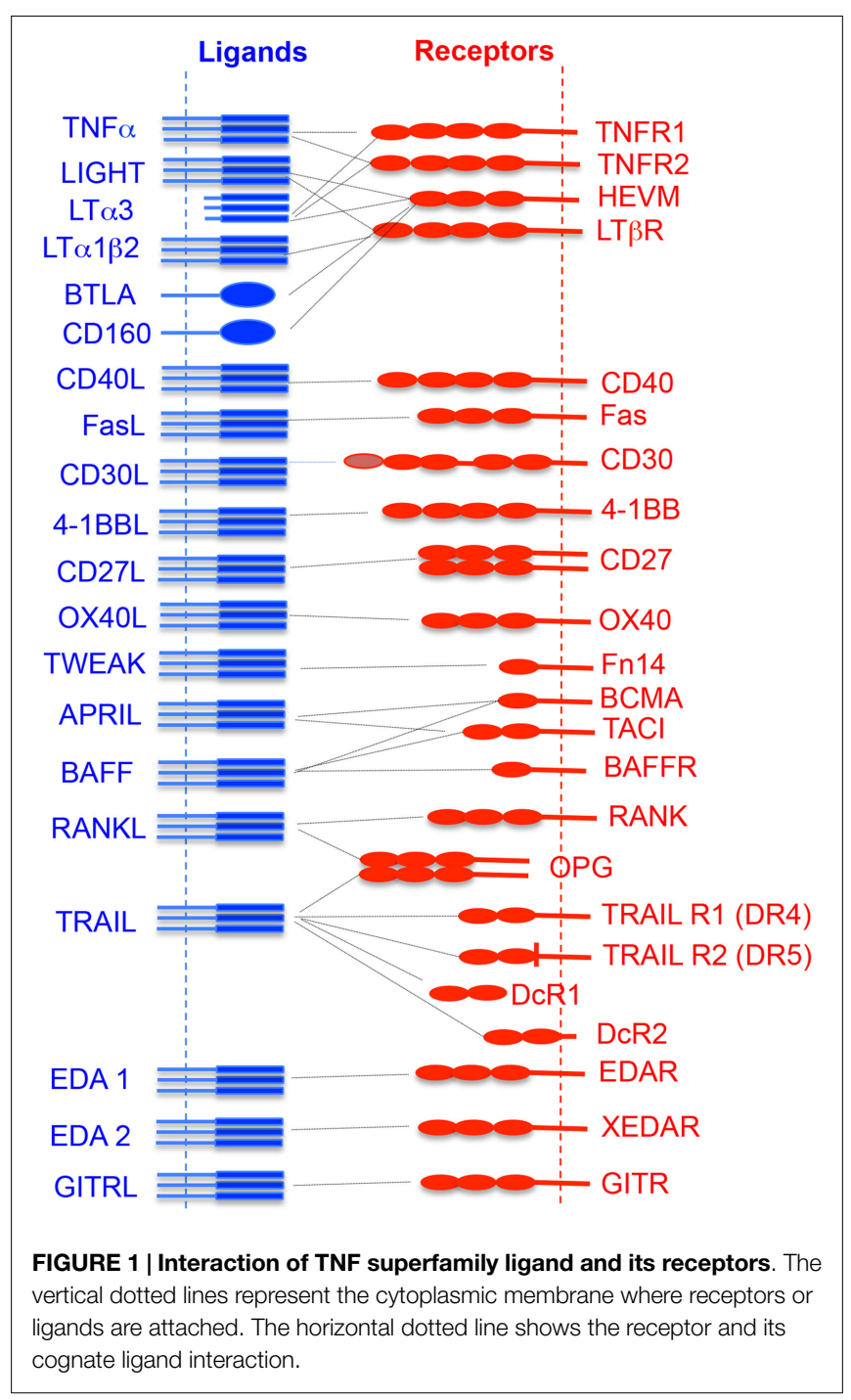

TNF receptor superfamily members are transmembrane proteins having cysteine-rich motifs in their extracellular domains that bind to their cognate ligands (Figure 1). About 30 members of TNFRSF have been identified $(3,6,7)$. Depending upon the specific intracellular signal induced by TNFRSF members, they can be categorized into three groups - death domain (DD)-containing receptors, decoy receptors, and TNF receptor-associated factor (TRAF)-binding receptors (8). Some TNFRSFs such as TNFR-1, Fas, DR3, DR4, DR5, and DR6, contain their own DDs. However, they also interact with other cytoplasmic DD-containing adaptor molecules (9). This receptor-adaptor complex acts as a scaffold for binding of immature pro-caspase, which then undergoes auto-cleavage, leading to the formation of the deathinducing signaling complex (DISC) and induction of apoptosis $(9,10)$. Some other TNFRSFs, such as TNFR-2, CD27, CD30, CD40, glucocorticoid-induced TNFR family-related gene (GITR), Fn1, lymphotoxin beta-receptor (LT $\beta R$ ), OX40, receptor activator of NF- $\kappa B$ (RANK), and XEDAR, lack a DD and contain motifs with four to six amino acids called TRAF-interacting motifs (TIMs) which recruits TRAF proteins. TRAF proteins are adaptor molecules that activate multiple downstream signaling pathways such as NF- $\kappa \mathrm{B}$, Janus kinase (JNK), ERK, p38MAPK, and $\mathrm{PI} 3 \mathrm{~K}$ that help in cell survival, proliferation, and cytokine production (11).

There are at least five anti-TNF medications (Etanercept, Infliximab, Adalimumab, Golimumab, and Certolizumab) approved by the U.S. Food and Drug Administration (FDA) for the treatment of rheumatoid arthritis (RA). Most of these biologics bind to soluble TNF molecules and prevent their binding to TNF-receptors. This blocks the production of pro-inflammatory cytokines such as IL-1, IL-6, or IFN- $\gamma$. Anti-TNF antibodies can also bind to surface-expressed TNF molecules and induce reverse signaling or antibody-induced cell death (AICD) $(12,13)$. In this review, we discuss the role of TNFSF-TNFRSF members that play a role in neuronal inflammation, the possible molecular mechanisms involved, and the efficiency of these molecules in controlling central nervous system (CNS) inflammation and autoimmunity.

The CNS is considered as an immune-privileged site and consists of a network of CNS microvessels. These microvessels are formed by a highly specialized endothelial lining supported by astrocytes, pericytes, microglial cells, and neurons, which together form a very firm blood-brain barrier (BBB). The BBB restricts entry of immune cells into the CNS, thereby actively maintaining a homeostasis. However, under inflammatory conditions, the $\mathrm{BBB}$ gets disrupted and immune cells migrate into the CNS parenchyma. A disrupted $\mathrm{BBB}$ is one of the hallmarks of autoimmune demyelinating diseases like MS and EAE (14-16).

Watts et al. have reported that vascular endothelial growth factor receptor (VEGFR) signaling activates JNK and positively regulates the angiogenesis and barrier property of $\mathrm{BBB}$ endothelial cells (17). They also reported that death receptor 6 (TNFRSF21) and TROY (TNFRSF19) were regulated in the acquisition and development of barrier property in BBB (17). Both these TNFRSF members are downstream targets of the Wnt/beta-catenin signaling pathway in the BBB endothelial cells. Dysregulation of TNFRSF21/TNFRSF19 signaling leads to the 
TABLE 1 | TNF superfamily in neuroinflammation.

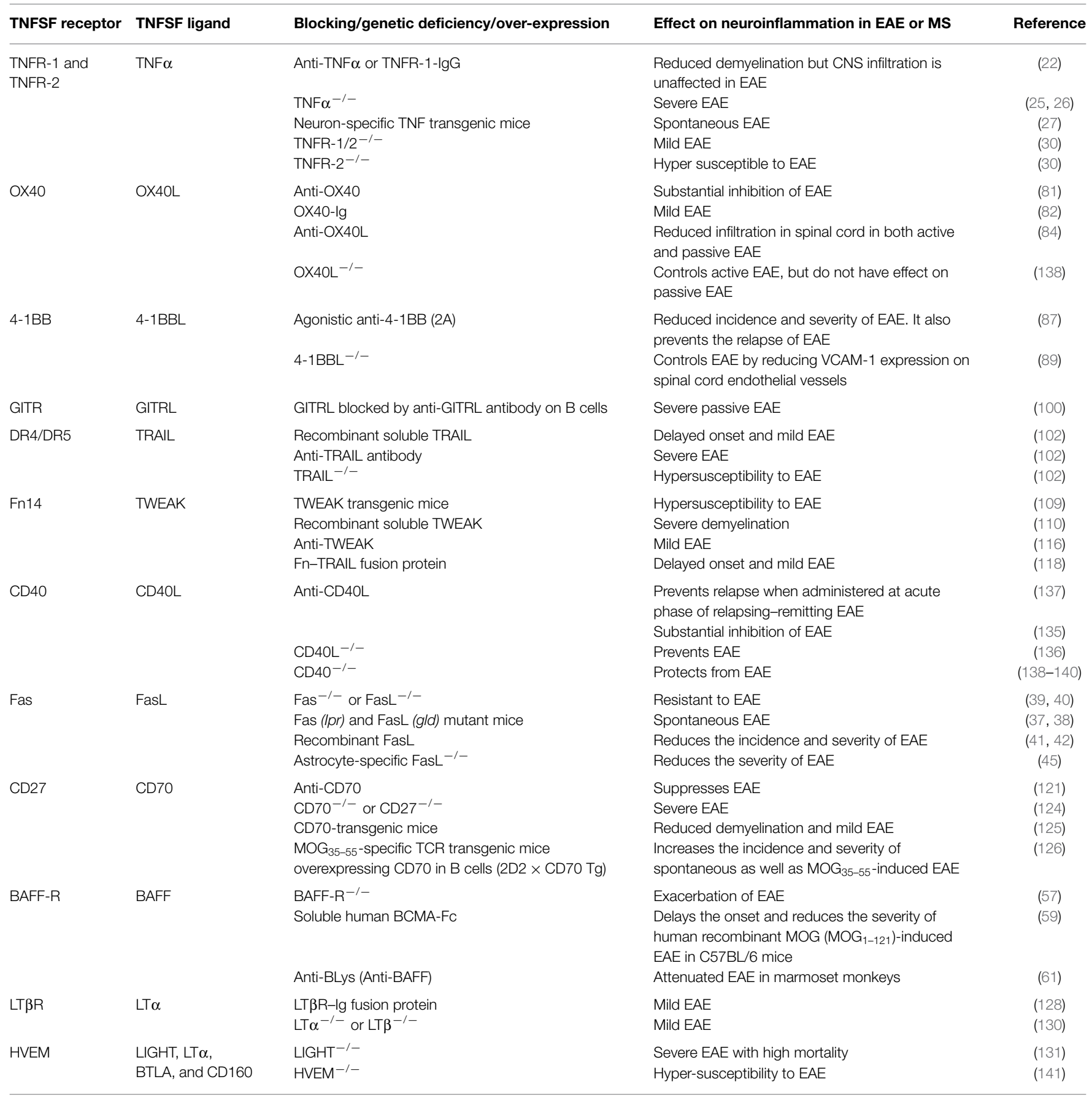

disruption of endothelial BBB. Since Wnt/beta-catenin signaling is required for CNS angiogenesis but not for peripheral vasculature $(18,19)$, an understanding of the molecular mechanism of this signaling would help in designing novel therapeutics or biologics that target TNFSF-TNFRSF interactions, to control CNS autoimmunity.

The importance of TNF superfamily receptors and ligands in neuroinflammation are listed in Table 1 . Some of the receptor-ligand interactions and their function at BBB (Figure 2) and in the brain parenchyma (Figure 3 ) are depicted. The role of important TNFSF-TNFRSF pairs in neuroinflammation and autoimmunity are discussed in details below.

\section{TNFR-TNF $\alpha$}

Tumor necrosis factor alpha (TNF $\alpha$ or TNFSF2) is a homotrimeric transmembrane protein that plays an important role in systemic inflammation. TNF $\alpha$ is expressed as a membranebound precursor $(\operatorname{tmTNF} \alpha)$, which is later cleaved between $\mathrm{Ala}^{76}-\mathrm{Val}^{77}$ by a metalloproteinase known as TNF $\alpha$-converting 


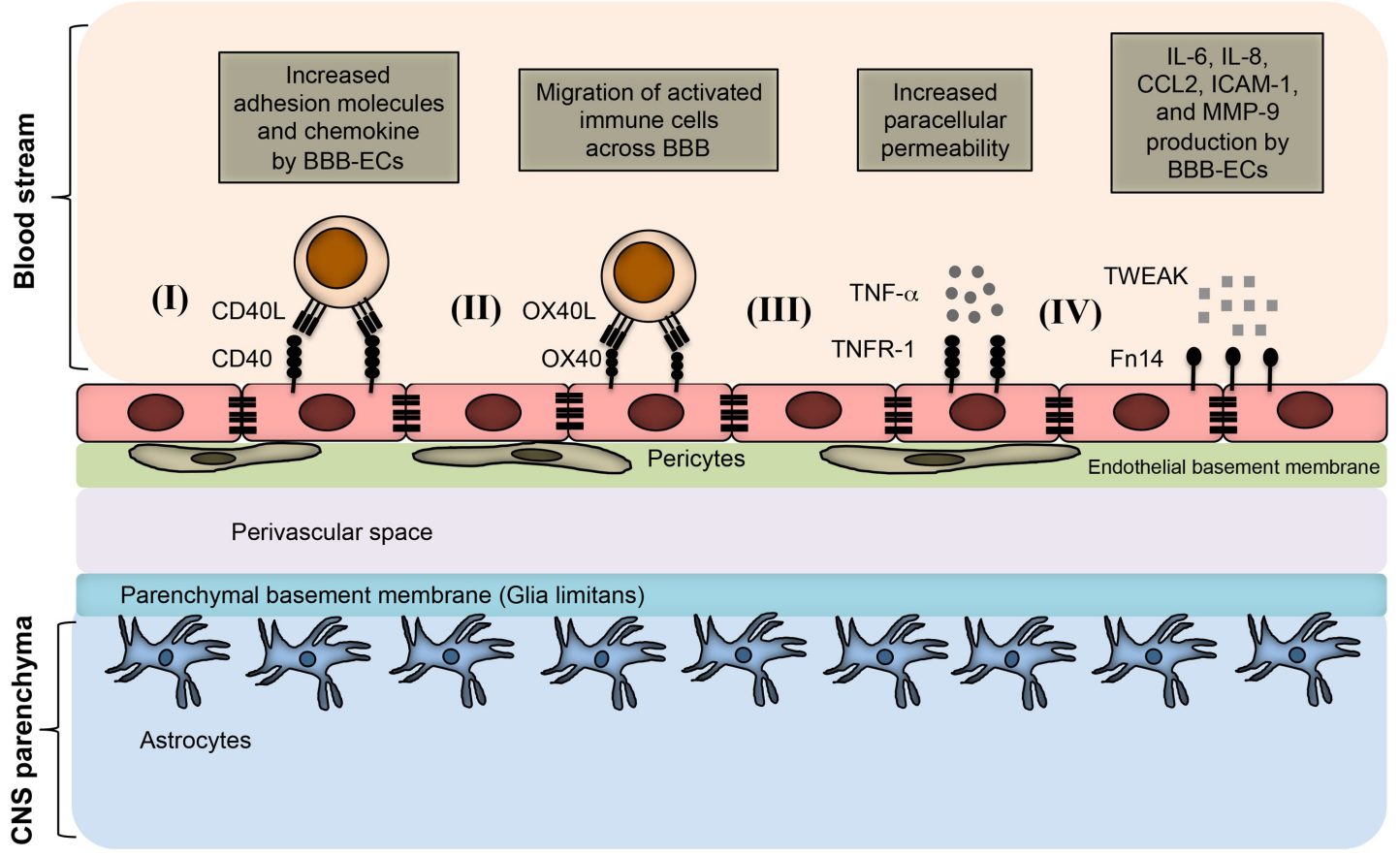

FIGURE 2 | TNFSF receptor-ligand interaction at endothelial BBB during neuroinflammation. BBB endothelial cells express TNFSF receptors during inflammatory condition, and interact with the TNFSF ligand in soluble form as well as on infiltrating immune cells. (I) Inflamed BBB endothelial cells express CD40. Interaction of CD40 with CD40L-expressing activated immune cells leads to up-regulation of adhesion molecules and chemokine secretion by BBB endothelial cells. This promotes the migration of pathogenic immune cell subsets into the CNS parenchyma. (II) OX40 expression can be induced in BBB endothelial cells during inflammation, which facilitates the migration of $\mathrm{OX}_{40 \mathrm{O}}{ }^{+}$ immune cells across the BBB. (III) Under inflammatory conditions, BBB endothelial cells up-regulate TNFR-1, which bind to soluble TNF secreted from various immune cells, such as activated Th1 cells, B cells, macrophages, and NK cells. Binding of TNF with TNFR-1 increases the paracellular permeability of BBB endothelial vessels. (IV) Inflamed BBB endothelial cells express Fn14 that binds to soluble TWEAK molecules. This leads to the up-regulation of cytokines, chemokines, cell adhesion molecules, and matrix metalloprotenase-9 (MMP-9). Increased expression of CCL2 and ICAM-1 facilitates the migration of pathogenic immune cells; whereas MMP-9 helps in the degradation of laminin molecules present in the basement membrane, resulting in loosening of the BBB. enzyme (TACE), and released as soluble TNF $\alpha$ (sTNF $\alpha$ ). TNF $\alpha$ is produced by many cell types, including activated macrophages, dendritic cells, monocytes, NK cells, $\mathrm{CD} 4^{+} \mathrm{T}$ cells, $\mathrm{CD} 8^{+} \mathrm{T}$ cells, astrocytes, and microglia (20). TNF $\alpha$ interacts with two receptors; TNF receptor type-1 (TNFR-1, also known as CD120a, p55/60) and TNF receptor type-2 (TNFR-2, also known as CD120b, p75/80). Low and constitutive expression of TNFR-1 is found on almost all nucleated cells, and can be activated by both membrane-bound TNF $\alpha(\operatorname{tmTNF} \alpha)$ and sTNF $\alpha$. However, expression of TNFR-2 is inducible and restricted to myeloid and lymphoid lineages. TNF $\alpha$ acts as a pro-inflammatory cytokine during the early phase of EAE, but shows immunosuppressive properties in the later phase. To be biologically functional, tmTNF $\alpha$ and $s T N F \alpha$ monomers must form homodimers. While both tmTNF $\alpha$ and sTNF $\alpha$ can bind to TNFR-1, sTNF $\alpha$ interacts with higher affinity than TNFR-2, leading to an inflammatory response. The tmTNF $\alpha$ mainly interacts with TNFR-2 and promotes cell survival (21). Blocking of TNF $\alpha$ by the administration of a soluble TNFR-1-IgG fusion protein or neutralization of anti-TNF $\alpha$ antibody has been shown to control the development of EAE (22). Interestingly, these biologics prevented demyelination but did not control the infiltration of immune cells into the CNS (22). Furthermore, such regimens also reduced the activation of $\mathrm{CD}^{+}{ }^{+} \mathrm{T}$ cell and microglia cells, and prevented demyelination in CNS $(23,24)$. While Korner et al. reported that a deficiency of TNF $\alpha$ only delays the disease progression and resulted in disease of comparable severity (25), others have reported that $\mathrm{TNF} \alpha$-deficient mice developed a more severe myelin oligodendrocyte glycoprotein (MOG) peptideinduced EAE as compared to wild-type littermate controls (26). Expression of TNF $\alpha$, specifically in neurons in the transgenic mice led to the development of spontaneous inflammatory demyelination (27). It has been reported that deficiency of TNF $\alpha$, LT- $\alpha$, and TNFR-1 or neutralization of TNF- $\alpha$ and LT $\alpha$ with monoclonal antibodies greatly reduced the severity of EAE (28-30). In contrast, TNFR-2-deficient mice developed more severe inflammation and demyelination in $\mathrm{MOG}_{35}-55$-induced EAE (30). Specific expression of tmTNF $\alpha$ in the transgenic mice showed reduced initiation and severity of the EAE, suggesting that selective targeting of sTNF $\alpha /$ TNFR-1 signaling may be helpful in controlling CNS autoimmunity.

In MS patients, TNF $\alpha$ were found to be expressed at very high levels in the CNS lesions and cerebrospinal fluids, but not in serum $(23,24)$, suggesting that TNF $\alpha$ was produced locally in the inflamed CNS. This indicates that TNF $\alpha$ might be a good target for therapy against MS. However, while TNF $\alpha$ 


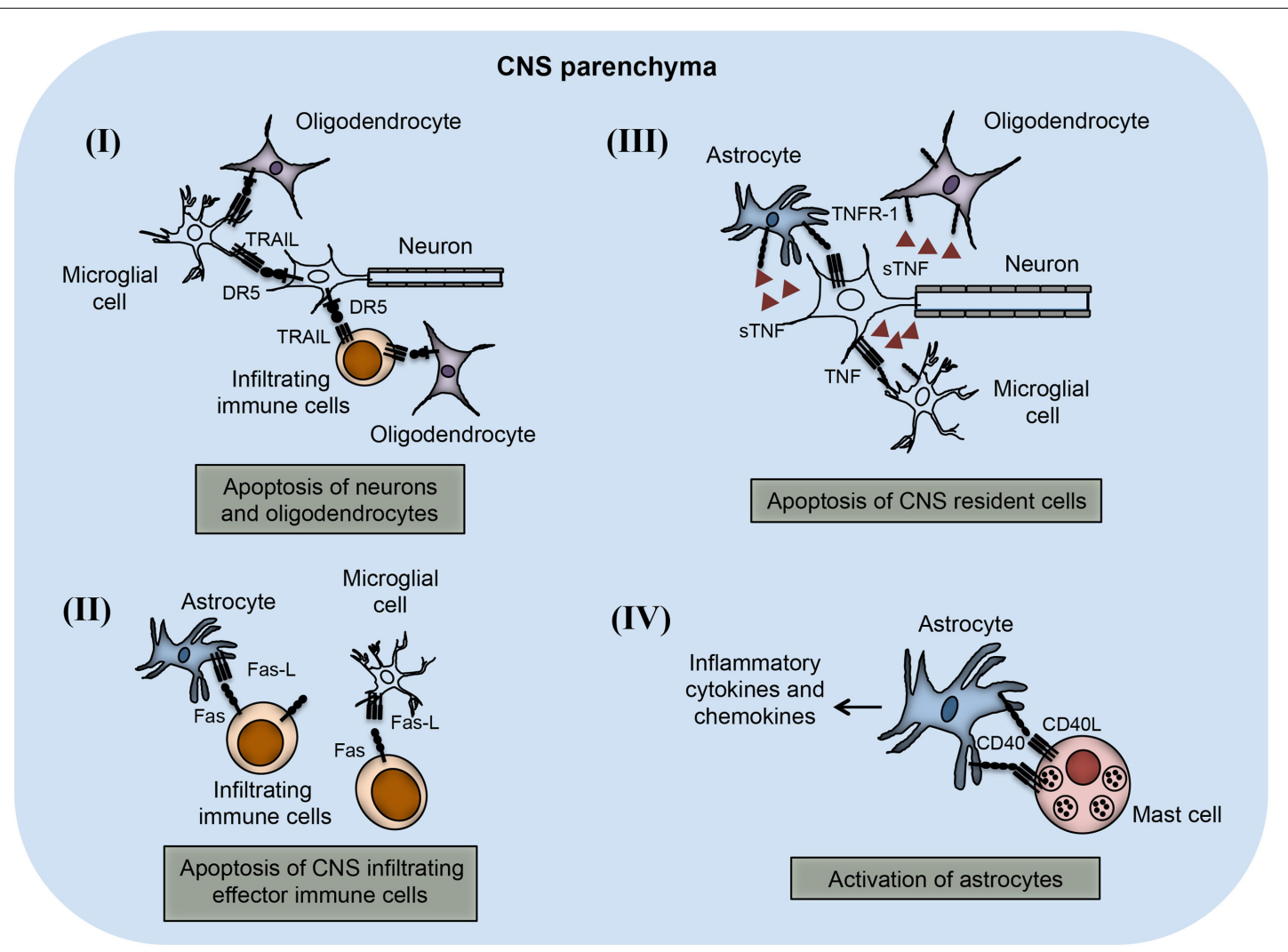

FIGURE 3 | TNFSF receptor-ligand interaction in the CNS parenchyma during neuroinflammation. TNFSF receptors and ligands are expressed on both CNS infiltrating effector immune cells and CNS-resident cells. The interaction of this receptor-ligand greatly influences the outcome of neuroinflammatory disease like multiple sclerosis and EAE. (I) Both neurons and oligodendrocytes express functional DR5 in the CNS during EAE. DR5 on the neurons as well as on oligodendrocytes interacts with TRAIL molecules present on either microglial cells or infiltrating immune cells, leading to apoptosis of DR5-expressing cells. (II) Activated astrocytes and microglial cells up-regulate FasL expression on their surface. The inhibitors showed protection in the mouse model of EAE, adverse effects were observed during clinical trials in MS patients (21). Similarly, a clinical trial with TNFR-1 fusion protein in MS patients showed disease exacerbation, and the trial was stopped (31). This increased demyelination with TNF $\alpha$ antagonist might be due to the several possible reasons such as: (a) down-regulation of anti-inflammatory cytokine IL-10 and increased production of inflammatory cytokine IL-12 and IFN- $\gamma(32,33)$; (b) downregulation of TNFR-2, which is known to regulate the proliferation of oligodendrocytes and damage repair (34); (c) possibility of reduced or non-permeability of the BBBs to TNF $\alpha$ blockers which would prevents their direct action through these molecules could still enhance the disease by increasing auto-reactive $\mathrm{T}$ cells in the peripheral tissues, which can migrate into the CNS and damage the tissues (35); (d) unmasking of latent infection, which is critical for inducing the autoimmune demyelination reaction (36). AntiTNF $\alpha$ therapy has also been used to control other autoimmune diseases such as RA. However, keeping in mind the possibility of demyelination of the central and peripheral neuronal tissues by interaction of FasL with Fas-expressing cells leads to apoptosis and elimination of pathogenic effector immune cells. (III) Neuronal cells express TNF and that can interact with TNFR-1 present on various CNS-resident cells, such as astrocytes, microglial cells, and oligodendrocytes. Interactions of TNF with TNFR-1-expressing cells lead to apoptosis of TNFR-1 ${ }^{+}$cells. (IV) Mast cells are known to localize close to the astrocytes during EAE in the brain. CD40L present on mast cells interact with CD40-expressing astrocytes, which induces increased production of inflammatory cytokines and chemokines. Local production of inflammatory molecules can augment inflammation and tissue damage in the CNS. such therapy, caution must be exercised, and careful monitoring of any pre-existing neuronal disease or its development following treatment with TNF $\alpha$ antagonists would be prudent.

\section{Fas-FasL}

Fas ligand (FasL, also known as CD95L, TNFSF6) is a type II transmembrane protein expressed on a variety of cells, including CD8 ${ }^{+}$ $\mathrm{T}$ cells and oligodendrocytes. It binds to the Fas receptor (Fas, also known as CD95, TNFRSF6, apoptosis antigen 1) and the decoy receptor 3 (DcR3). FasL-Fas interactions lead to the formation of a DISC in the Fas-expressing cells, leading to induction of apoptosis. The interaction of Fas with FasL on CNS infiltrating cells or activated CNS-resident cells (microglia, astrocytes, and neurons) also leads to the induction of apoptosis. FasL-Fas interactions play a very important role in immune cell homeostasis, and its dysregulation leads to various autoimmune diseases. Mice with autosomal recessive mutations in Fas (lymphoproliferative, lpr mice; lack Fas expression) and FasL (generalized lymphoproliferative disease, gld 
mice; lack FasL expression) genes develop a spontaneous autoimmune syndrome; produce autoantibodies and accumulate a large number of $\mathrm{CD}^{-} \mathrm{CD}^{-} \mathrm{T}$ cells in the secondary lymphoid tissues, leading to the progressive development of lymphadenopathy and splenomegaly $(37,38)$. Fas and FasL-deficient mice are resistant to MOG-induced EAE, compared to wild-type mice $(39,40)$, and intrathecal injection of recombinant FasL protein or neutralizing with anti-FasL antibody suppresses acute EAE $(41,42)$. Adoptive transfer of myelin basic protein (MBP)-specific FasL ${ }^{-1-} \mathrm{CD} 4$ $\mathrm{T}$ cells in the wild-type host showed reduced EAE pathology suggesting that FasL expression regulates the encephalitogenic function of $\mathrm{T}$ cells (43). It would be interesting to study how these auto-reactive $\mathrm{CD}^{+}{ }^{+} \mathrm{T}$ cells cross $\mathrm{BBB}$ and cause neuronal tissue damage. Determining the temporal and site-specific expression of FasL in a variety of CNS-resident cells during ongoing neuroinflammation might provide valuable clues to address this question. It has been also reported that Lpr mice on SJL/J background are completely susceptible to the proteolipid protein ( $\left.\mathrm{PLP}_{139-51}\right)$ induced EAE (40). This discrepancy might be due to the involvement of other effector pathways mediated by various TNFRSF members in the CNS.

Astrocytes constitutively express FasL, and they show both positive and negative roles in the inflammation and the development of EAE (44). Using targeted deletion of FasL, specifically in astrocytes, Wang et al. showed that astrocytic FasL is required for the elimination of auto-reactive $\mathrm{T}$ cells in the CNS (45). Gamma-delta $(\gamma \delta) \mathrm{T}$ cells play a very important role in EAE (46), and their deficiency results in chronic EAE (47). $\gamma \delta$ T cells were shown to regulate CNS inflammation and disease recovery in a FasL-Fas-dependent manner by controlling the encephalitogenic $\mathrm{CD}^{+}{ }^{+} \mathrm{T}$ cells (47). Collectively, these reports suggest that Fas-FasL-mediated apoptosis acts as an intrinsic regulatory mechanism to control neuroinflammation and development of CNS autoimmunity.

\section{BAFF-R-BAFF}

The B-cell activating factor of the tumor necrosis factor family (BAFF; also known as TNFSF13B, CD257, B lymphocyte stimulator "BLys"), was originally described as a molecule secreted by $\mathrm{T}$ cells and dendritic cells that provide maturation and survival signals to peripheral B cells (48). Its expression was also reported in other cell types such as macrophages, dendritic cells, and neutrophils (49). BAFF transgenic mice have elevated numbers of $\mathrm{B}$ cells and effector $\mathrm{T}$ cells, and show symptoms similar to that seen in B-cell-mediated autoimmune diseases (50). Immature $\mathrm{B}$ cells in the bone marrow express BAFF-receptor (BAFF-R), and its expression is also up-regulated during the development from transitional B-cell stage to mature B cell in the secondary lymphoid organs $(51,52)$. BAFF also interacts with two more known receptors expressed on the $B$ cells; transmembrane activator calcium modulator and cyclophilin ligand interactor (TACI) and B-cell maturation factor (BCMA) (53). TACI is known to be expressed on marginal zone $\mathrm{B}$ cells, and BCMA on germinal center B cells, plasma cells, and memory B cells (54). In addition to BAFF, TACI and BCMA are also known to interact with another
TNFSF member known as APRIL $(55,56)$. Genetic deficiency of BAFF-R in mice resulted in hyper-susceptibility to $\mathrm{MOG}_{35-55}$ peptide-induced EAE (57), suggesting that this receptor has a regulatory function in EAE. It has been reported that defects in the BAFF-BAFF-R signaling adversely influence the regulatory Bcell (Breg) function, inducing early onset and severe pathology of the disease (58). Huntington et al. showed that administration of $\mathrm{hBCMA}-\mathrm{Fc}$ fusion protein in C57BL/6 mice not only reduced the $\mathrm{B}$-cell numbers in peripheral blood, lymph nodes, and spleen, and resulted in a reduced titer of MOG-specific antibody in the serum but it also hampered the activation and differentiation of $\mathrm{CD} 4^{+}$ $\mathrm{T}$ cells (59). It has also been reported that BAFF can enhance the auto-reactive Th17 response, leading to increased progression of EAE (60). Antibody-mediated blocking of BAFF with anti-human BLys attenuated the EAE in marmoset monkeys (61). Glatiramer acetate (GA) is the approved frontline drug for the treatment of EAE and MS, which acts on both innate as well as $\mathrm{T}$ and $\mathrm{B}$ cells during EAE, and induces an anti-inflammatory microenvironment in the neuronal tissue (62-65). GA was also shown to reduce BAFF mRNA expression in the brain, and decrease the number of BAFF- ${ }^{+} \mathrm{B}$ cells, but not the $\mathrm{TACI}^{+} \mathrm{B}$ cells in the spleen of EAE mice (66). These reports clearly indicate that BAFF-BAFF-R signaling contributes to the pathogenesis of EAE, and its perturbation may provide a valuable therapeutics tool to control CNS autoimmunity.

\section{OX40-OX40L}

The OX40 (also known as ACT35, CD134, TNFRSF4) is a type I glycoprotein of $\sim 50 \mathrm{kD}$, which is expressed on activated $\mathrm{T}$ cells, such as Th1, Th2, Th17, Foxp $3^{+}$regulatory CD4 ${ }^{+} \mathrm{T}$ cell (Treg), and $\mathrm{CD}^{+} \mathrm{T}$ cells (67). The OX40 ligand (OX40L also known as gp34, CD252, TNFSF4) is a type II glycoprotein expressed on APCs, such as dendritic cells, B cells, macrophages, and endothelial cells (68). The interaction of OX40-OX40L provides a costimulatory signal to $\mathrm{T}$ cells, which leads to their activation and cytokine secretion (69). Inhibiting OX40-OX40L interactions protects from many inflammatory autoimmune diseases, including $\mathrm{EAE}(70,71)$. OX40-OX40L signaling in $\mathrm{CD}^{+}{ }^{+} \mathrm{T}$ cells promotes the expression of IL-12R $\beta 2$ (72) while inhibits the expression of CTLA-4 (73), Foxp3 (74-76), and IL-10 (77). Selective up-regulation of IL-12R $\beta 2$ leads to the differentiation of Th1, whereas loss of Foxp3, CTLA-4, and IL-10 inhibits the suppressive function of Tregs. Thus, the loss of balance between pathogenic and regulatory cells could promote autoimmune pathology. A growing body of evidences suggests that OX40-OX40L interactions regulate the differentiation of $\mathrm{CD}^{+}{ }^{+} \mathrm{T}$-cell subsets, and deficiency of OX40 impairs Treg development (78). This might be because the reverse signaling through OX40L in APCs leads to the production of cytokines IL- 6 and IL-12, which regulates the differentiation of effector CD4 ${ }^{+} \mathrm{T}$ cells (68). It has been shown that OX40L-OX40 signaling inhibits Foxp3 expression $(75,79)$, and acts as a strong differentiating factor for Th9 in airway inflammation (80). These studies suggest that OX40-OX40L signaling could promote inflammatory responses, in addition to suppressing anti-inflammatory responses in $\mathrm{CD}^{+} \mathrm{T}$ cells $(68,78)$. All 
these observations suggest that functional plasticity of the $\mathrm{CD} 4^{+}$ T-cell subsets is also controlled by OX40L-OX40 interactions.

OX40-OX40L interactions in CD28-deficient mice initially led to the identification of OX40-OX40L signaling as being costimulatory in EAE, and blocking these interaction-protected mice from EAE (71). In the rat EAE model, pathogenic OX40 ${ }^{+}$ $\mathrm{CD}^{+}{ }^{+} \mathrm{T}$ cells were found in CNS lesions, and neutralization of OX40 by anti-OX40 antibody ameliorated the EAE (81). Targeting OX40-OX40L interaction with an OX40-Ig fusion protein at the onset of EAE greatly reduced the severity of the disease (82). Brain endothelial cells are the central component of the BBB and are known to express OX40L, which recruit $\mathrm{OX} 40^{+}$auto-reactive T cells into the CNS (82). OX40 treatment of human umbilical cord endothelial cells (HUVECs) lead to the up-regulation of CCL5, suggesting that this signaling also modulates endothelial cells to attract a selective population of immune cells at the BBB (83). It would be interesting to study whether OX40 signaling affects the expression of cell adhesion molecules, chemokines, and integrins in the brain endothelial cells. Blocking OX40L by anti-OX40L antibody reduces the infiltration of OX40 ${ }^{+}$myelinspecific $\mathrm{T}$ cells in the spinal cord without affecting the priming of the myelin-specific $\mathrm{CD} 4^{+} \mathrm{T}$ cells in the draining lymph nodes in active as well as passive EAE (84). Therefore, OX40-OX40L not only controls the activation and differentiation of $\mathrm{CD} 4^{+} \mathrm{T}$ cells, but also potentiates their migration into the CNS. Together, these reports indicate that OX40-OX40L supports the development of neuroinflammation and CNS autoimmunity.

\section{4-1BB (CD137) - 4-1BBL (CD137L)}

4-1BB (TNFRSF9) is expressed on activated CD4 ${ }^{+} \mathrm{T}$ cells (Th1, Th2, and Treg), CD8 ${ }^{+} \mathrm{T}$ cells, B cells, dendritic cells, NK cells, NKT cells, and mast cells, whereas its ligand, 4-1BBL, is expressed on activated APCs (macrophages, B cells, and dendritic cells), $\mathrm{CD}^{+}{ }^{+}$and $\mathrm{CD}^{+}{ }^{+} \mathrm{T}$ cells, NK cells, mast cells, and smooth muscle cells (85). Under inflammatory condition, neurons are also known to express 4-1BBL (3). B7-deficient APCs deliver the costimulatory signal to $\mathrm{CD} 4{ }^{+} \mathrm{T}$ cells through $4-1 \mathrm{BBL}$ in a TRAF2dependent manner (86). It has been reported that stimulation of human $\mathrm{CD}^{+} \mathrm{T}$ cells with 4-1BBL leads to the production of effector molecules such as perforin and granzyme (3). Antibody treatment with agonist anti-4-1BB (2A) in induced EAE resulted in milder disease, but failed to control the development of passive EAE by adoptive transfer of $\mathrm{MOG}_{35-55}$-specific $\mathrm{CD}^{+} \mathrm{T}$ cells (87). Anti-4-1BB (2A) treatment induced IFN- $\gamma$ and granulocyte-macrophage colony-stimulating factor (GM-CSF) secretion by $\mathrm{MOG}_{35-55}$-specific $\mathrm{CD}^{+}{ }^{+} \mathrm{T}$ cells, leading to differentiation of inflammatory Th1 (87). It has been suggested that targeting 4-1BB or 4-1BB ligand could lead to the induction of unresponsiveness in the $\mathrm{CD}^{+}$T cells during EAE $(88,89) .4-1 \mathrm{BBL}^{-1-}$ mice showed reduced expression of vascular cell adhesion molecule-1 (VCAM1) on spinal cord endothelial vessels, which play an important role in the migration of immune cells in the inflamed CNS (89). 4$1 \mathrm{BBL}$ downstream signaling induced the production of reactive oxygen species (ROS) in microglia, leading to apoptosis of oligodendrocytes in the EAE (85). Therefore, these studies indicate that
4-1BB-4-1BBL interactions not only promote the activation of $\mathrm{T}$ cells, but also control the migration of myelin-specific $\mathrm{CD} 4^{+} \mathrm{T}$ cells in the CNS. Furthermore, 4-1BBL signaling has a destructive role in the inflamed CNS during EAE.

\section{GITR-GITRL}

Glucocorticoid-induced TNFR family-related gene (also known as CD357, TNFRSF18) is expressed at the low levels in resting mouse and human T cells, but is up-regulated on activated CD4 ${ }^{+}$ and $\mathrm{CD}^{+}{ }^{+} \mathrm{T}$ cells. GITR is constitutively expressed on Tregs $(90,91)$. GITR-GITRL interactions inhibit the suppressive function of $\mathrm{CD}^{+}{ }^{+} \mathrm{CD} 25^{+}$Tregs and therefore, blocking these interactions breaks the peripheral immune tolerance (92). GITRL is expressed at the low levels on B cells, macrophages, bone marrowderived dendritic cells, and endothelial cells (3). GITRL can act as a costimulatory signal to $\mathrm{CD} 4{ }^{+} \mathrm{CD} 25^{-}$and $\mathrm{CD} 4{ }^{+} \mathrm{CD} 25^{+} \mathrm{T}$ cells (93), and to $\mathrm{CD}^{+} \mathrm{T}$ cells in the presence of suboptimal concentrations of anti-CD3 $\varepsilon$, in the absence of CD28-mediated signaling (94). In addition, signaling through GITR stimulates the production of cytokines such as IFN- $\gamma$, IL-2, IL-4, and IL-10 in $\mathrm{CD}^{+}{ }^{+} \mathrm{T}$ cells (95).

Regulatory B cells are known to produce anti-inflammatory cytokine IL-10 (96) and suppress CNS autoimmunity (97). Transgenic mice constitutively expressing GITRL on B cells (GITRL $^{+}$B cells) showed a significantly higher numbers of peripheral Tregs, suggesting that GITRL ${ }^{+} \mathrm{B}$ cells might play a role in the homeostasis of Tregs (99). Animals that received, B cells pre-treated with anti-mouse GITRL antibody showed significantly decreased Tregs induction and severe EAE, suggesting that GITRL ${ }^{+}$B cells support the proliferation and homeostasis of Tregs (100). Treatment with Rituximab, a monoclonal antibody that depletes B cells, including Bregs, leads to severe exacerbation of human ulcerative colitis, suggesting that Bregs play an important role in controlling CNS autoimmunity (98). A peripheral increase in Treg frequency can inhibit the proliferation of myelinspecific effector $\mathrm{CD} 4^{+} \mathrm{T}$-cell subsets in the secondary lymphoid organs, and can therefore control CNS autoimmunity. This clearly indicates that B cells, through the expression of GITRL, promote and maintain the expansion of Tregs and contribute to the maintenance of immune tolerance.

\section{DR4/5-TRAIL}

TNF-related apoptosis-inducing ligand (also known as CD253, TNFSF10) is a type II membrane protein that binds to two death receptors, DR4 (TRAIL-RI) and DR5 (TRAIL-RII). These receptors are known to induce apoptosis in various cell types in a caspase-dependent manner. Both DR4 and DR5 are known to be expressed in humans, whereas mice express only DR5 (101). TRAIL also binds to the decoy receptors, DcR1 (lack cytoplasmic domain) and DcR2 (truncated DD). Rather than inducing apoptosis, binding to these receptors induces $\mathrm{NF} \kappa \mathrm{B}$ activation. TRAIL receptors are expressed in neurons and oligodendrocytes, but TRAIL is completely undetected in a healthy CNS. Signaling from TRAIL-DR4/5 is implicated in the pathogenesis of MS and EAE (101). TRAIL is generally found on infiltrating 
immune cells and activated microglia in the MS lesions. The DR4/DR5-TRAIL interaction seems to induce oligodendrocyte and neuronal death during ongoing EAE. TRAIL-induced apoptosis of neurons and oligodendrocytes contribute to the development of brain inflammation. Blocking brain-specific TRAIL leads to a reduction in the severity of EAE. Similarly, therapeutic treatment with soluble TRAIL delays the onset of the disease and reduces its severity (102). TRAIL is also reported to inhibit Th1 response and promote the suppressive function of Tregs (103). Genetically modified dendritic cells (ES-DCs), which can simultaneously present MOG peptides in association with MHC-II and express TRAIL, cause a reduction in the severity of EAE induced by both MOG $_{35-55}$ and MBP $(104,105)$. One of the mechanisms through which these ES-DCs controls EAE is by promoting the proliferation of $\mathrm{CD} 4{ }^{+} \mathrm{CD} 25^{+}$Tregs, which suppress myelin-specific effector $\mathrm{CD} 4^{+} \mathrm{T}$-cell responses. It might be possible that TRAIL signaling has a dual role; it may promote suppressive phenotype in the secondary lymphoid organs, whereas in local inflamed CNS, it contributes to tissue damage. Therefore, delineating the role of TRAIL signaling in a spatio-temporal manner during the neuroinflammatory events might help in understanding the complexity of CNS autoimmunity. Osteoprotegerin (OPG), a secreted protein under physiological conditions, shows a lower binding affinity for TRAIL (106); however, its role in CNS inflammation and autoimmunity is not well studied. All these studies suggest that, the apoptosis-inducing property of TRAIL in inflamed CNS contributes to the development of inflammation and CNS autoimmunity.

\section{TWEAK-Fn14}

TNF-like weak inducer of apoptosis (TWEAK; TNFSF12) is a pro-inflammatory and pro-angiogenic cytokine synthesized as a type II transmembrane protein. However, it can be cleaved to give rise to soluble cytokine (107). Both membrane-bound and soluble TWEAK bind to the only known receptor, fibroblast growth factor-inducible 14 (Fn14; also known as TNFRSF12A). TWEAK is expressed on monocytes, microglia, and astrocytes in the healthy CNS. Its expression goes up during CNS inflammation. Fn14 is also expressed on CNS-resident cells such as brain endothelial cells, astrocytes, and neurons $(108,109)$. Overexpression of soluble TWEAK by injecting TWEAK-encoding recombinant plasmid (110), or in TWEAK transgenic animals resulted in increased severity of EAE (109). These studies indicate that local expression of TWEAK and Fn14 in the CNS could be a critical contributing factor to the pathology of neuroinflammation. One of the mechanisms by which TWEAK elevates CNS inflammation is by inducing CCL2 secretion from brain endothelial cells and astrocytes. CCL2 is a potent activator of neuroinflammation, and plays an important role in the pathogenesis of EAE and MS $(111,112)$. TWEAK-Fn14 signaling in BBB endothelial cells compromises its barrier property in the mouse models of cerebral ischemia. Therefore, it is possible that during neuroinflammation such interactions can affect the $\mathrm{BBB}$, allowing the myelin-specific cells and soluble mediators to enter into the CNS parenchyma $(113,114)$.
TWEAK-Fn14 binding does not induce ligand-activated kinase signaling due to lack of cytoplasmic DD, but it triggers the engagement of the TRAF, an adaptor protein that activates the ERK1/2, PI3K/Akt, and NF- $\kappa B$ signaling pathways. Membranebound TWEAK is a more powerful inducer of the classical NF$\kappa \mathrm{B}$ signaling pathway than its soluble forms (115). It has been shown that inhibition of TWEAK-Fn14 signaling decreases the severity of EAE $(110,116)$ and CIA (117). Neutralization of TWEAK after the priming phase with monoclonal antibody controls immune cell infiltration into the neuronal tissues and reduce the pathology of EAE (116). An immunotherapeutic fusion protein, Fn14-TRAIL consists of a portion of Fn14 receptor fused with the TRAIL ligand, which blocks the function of TWEAK. In vivo expression of this soluble functional chimera was shown to control the EAE (118). A functional analysis of T-cell response in these mice showed decreased effector Th1 and Th17 responses, and increased number of suppressive Tregs, suggesting that the balance was shifted more toward immune tolerance, which controls the EAE (119). These studies suggest that TWEAK is a very good therapeutic target to control neuroinflammation and autoimmunity. Currently, several TWEAK-targeting therapeutic agents are in clinical trials for autoimmunity and cancer (107).

\section{CD70-CD27}

The CD70 (CD27L, also known as TNFSF7) is a homotrimeric type II transmembrane glycoprotein, and known to express on $\mathrm{B}$ cells, T cells, mast cells, NK cells, and activated dendritic cells. It is also expressed on epithelial cells in the thymic medulla. CD70 mainly resides in the endosomal compartment. Its expression is activation-dependent, and controlled by the master transcription regulator of MHC class II gene, CIITA. CD70 binds to its receptor CD27 (TNFRSF7) expressed on $\mathrm{CD}^{+}$and $\mathrm{CD}^{+}{ }^{+} \mathrm{T}$ cells, and provides a costimulatory signal, which leads to the proliferation and survival of activated B and T cells. The CD27-CD70 interaction also controls effector and memory responses, and prevents the induction of tolerance (120). Treatment of animals with antiCD70 antibody suppresses the EAE, possibly by inhibiting the TNF- $\alpha$ production by draining lymph node cells (121). CD70transgenic mice showed increased numbers of IFN- $\gamma$-producing $\mathrm{CD}^{+}{ }^{+}$and $\mathrm{CD}^{+} \mathrm{T}$ cells, suggesting that CD70-CD27 signaling controls Th1 (120). It has been reported that $\mathrm{CD} 27^{+} \gamma \delta$ $\mathrm{T}$ cells express high levels of IFN- $\gamma$ and lower levels of IL-17, whereas $\mathrm{CD}^{-} 7^{-} \gamma \delta \mathrm{T}$ cells express lower levels of IFN- $\gamma$ and high levels of IL-17, suggesting that CD27 essentially controls the differentiation of $\gamma \delta$ T cells, which gives rise to two different effector subsets of $\gamma \delta$ T cells $(46,122,123)$. The deficiency of CD27 and CD70 in animals leads to significantly increased EAE. In contrast, CD70-Tg mice showed reduced EAE pathology $(124,125)$. Furthermore, Coquet el al. reported increased Th17 response in $\mathrm{CD}_{27}^{-/-}$and $\mathrm{CD} 70^{-/-}$, and reduced Th17 in CD70Tg mice (124). They concluded that this increased Th17 differentiation in $\mathrm{CD} 27^{-1-}$ and $\mathrm{CD} 70^{-1-}$ animals were due to increased phosphorylation of the JNK and epigenetic modification at IL17 locus, suggesting that CD70-CD27 signaling directly controls the Th17 response in $\mathrm{CD}^{+} \mathrm{T}$ cells (124). Interestingly, antiCD70 antibody-mediated blocking of CD70 prevented EAE in 
SJL/J mice (121), and over-expression of CD70 on the B cells enhanced EAE (126). This is in contrast to what is reported by Coquet et al. (124). These discrepancies might be due to the differences in the mouse strains used or in the cell types where CD70-Tg was over expressed, since over-expression of CD70 in B cells leads to hyper-activation of T cells and a gradual loss of B cells (127). CD70 signaling affects the expression of IL-17F and CCR6 but not other Th17-associated molecules such as RORs, BATF, and IL-23R (124), suggesting that targeting CD27 may be beneficial in controlling effector CD4 ${ }^{+}$T-cell differentiation and its migration into the inflamed tissues during autoimmunity.

\section{LT $\beta R-L T \alpha$}

Lymphotoxin alpha (also known as TNFSF1) exists as a secreted homotrimeric molecule produced by lymphocytes. LT $\alpha$ also forms a membrane-anchored heterotrimer with LT $\beta$ (Figure 1). LT $\alpha$ homotrimer $\left(\mathrm{LT}_{3}\right)$ interacts with TNFR-1, TNFR-2, and herpes virus entry mediator (HEVM), whereas the heterotrimer molecule $\left(\mathrm{LT} \alpha_{1} \beta_{2}\right)$ interacts with the LT $\beta \mathrm{R}$ (also known as TNFRSF3) (Figure 1). LT $\beta \mathrm{R}$ is expressed on most cell types, including epithelial cells, endothelial cells, and cells of the myeloid lineages, but not on B and T lymphocytes. LT levels were known to increase in the CNS before the onset of clinical signs of EAE, suggesting a role in the pathogenesis of CNS inflammation. Treatment with LT $\beta \mathrm{R}-\mathrm{Ig}$ fusion protein alters the localization of leukocytic infiltration into the CNS and controls the EAE (128). $\mathrm{LT} \alpha^{-1-}$ mice have developmental defects in the lymph node formation, and lack Peyer's patches (129). LT $\alpha^{-1-}$ mice show reduced demyelination and $\mathrm{CNS}$ inflammation in $\mathrm{MOG}_{35-55^{-}}$ induced EAE. However, adoptive transfer of $\mathrm{MOG}_{35}-55$-specific wild-type T cells induces EAE (130). This indicates that LT-LT $\beta R$ signaling mainly regulates the priming phase of myelin-specific T-cell activation and development, but not the actual homing of these auto-reactive cells into the inflamed CNS. In contrast, $\mathrm{LT}^{-1-}$ mice develop $\mathrm{MOG}_{35-55}$-induced EAE, but to a somewhat lesser extent as compared to wild-type animals. These results suggest that LT $\alpha$ plays a very important role in the development of EAE (130). LIGHT (CD258, also known as TNFSF14) is a potent, CD28-independent costimulatory molecule expressed on $\mathrm{T}$ cells that are involved in initial T-cell priming and expansion. LIGHT can interact with both LT $\beta$ R and HEVM. Interaction of LIGHT with HVEM has a costimulatory function, whereas its interaction with LT $\beta$ R induces apoptosis. LIGHT-deficient mice develop more severe $\mathrm{MOG}_{35-55}$-induced EAE due to intensive activation of microglia/macrophages and increased frequency of apoptotic cells within the CNS parenchyma, as compared to wild-type animals (131). Expression of LIGHT on CNS-resident cells rather than myelin-specific $\mathrm{CD} 4^{+} \mathrm{T}$ cells is the most crucial factor in the pathogenesis of EAE. This also suggests that LIGHT expression plays an important role in controlling the activated microglia/macrophages during CNS inflammation. Therefore, selective targeting of LIGHT-HVEM signaling may provide protection against neuroinflammation and CNS autoimmunity. However, the involvement of LIGHT-HVEM interactions with other CNS-resident and infiltrating cells is not well characterized.
It would be interesting to study these interactions in different stages of neuroinflammation.

\section{CD40-CD40L}

The CD40 ligand (CD154, also known as TNFSF5) is expressed on activated $\mathrm{T}$ cells, and binds to its cognate receptor CD40 (TNFRSF5) on APCs. CD40L-CD40 interactions lead to the activation of $\mathrm{B}$ cells, their differentiation into plasma cells, and immunoglobulin class switching. The CD40L-CD40 interactions play a very important role in many autoimmune diseases. The disruption or blocking of this interaction inhibits clinical manifestation and ameliorates the EAE in mice and monkeys (132, 133). In the CNS, various cells, including astrocytes and glial cells express CD40, and expression of this molecule is required for the development of $\operatorname{EAE}(133,134)$. It has been reported that blocking of CD40L-CD40 interaction controls the development of $\operatorname{EAE}(135,136)$. Treatment of animals with remitting EAE in this manner not only controls the EAE, but also inhibits the longterm delayed type hypersensitivity (DTH) response (137). One study has reported that mast cells and astrocytes are localized together in the inflamed CNS, and they have potential to interact with each other via CD40L on mast cells and CD40 on astrocytes (134). In vitro studies have shown that this interaction activates astrocytes leading to the secretion of cytokines. These cytokines act in an autocrine manner, and potentiate multiple signaling via the JAK-STAT-1 $\left(\mathrm{Tyr}^{701}\right)$ pathway in the astrocytes (134). Therefore, a contributing signaling event that can enhance the function of infiltrating effector immune cells as well as CNSresident cells may also promote axonal damage. These reports suggest that CD40-CD40L costimulatory signaling contributes to neuroinflammation and CNS autoimmunity.

\section{Future Perspective}

The growing body of evidence demonstrated that TNF-TNFR interactions are involved in the pathogenesis of EAE and MS. These interactions control the disease outcome by fine-tuning the peripheral immune response as well as interactions between CNS-resident cells and effector immune cells in the CNS. Several reports suggest that the TNFSF-TNFRSF pairs that signal the promotion of inflammation are OX-40-OX40L, sTNF $\alpha-$ TNFR1, CD27-CD70, 4-1BB-4-1BBL, Fn14-TWEAK, CD40-CD40L, and LT- $\alpha / \mathrm{LT} \beta-\mathrm{LT} \beta \mathrm{R}$; whereas the ones that show a protective role are tmTNF $\alpha$-TNFR-2, DR4/DR5-TRAIL, and HVEM-LIGHT. Conflicting results have been reported in the literature about some of the interactions and their importance in CNS autoimmunity. These discrepancies in the results reported by different investigators may be due to the differences in the animal models used or immunization strategies employed to induce the EAE. Furthermore, cross-talks between the signaling induced by various TNFSF-TNFRSF may also contribute to the pathogenesis of disease. Keeping in mind that the combination therapy is central to immunotherapeutic approaches, understanding how and when to block TNFSF-TNFRSF interactions, individually or in combination with other targets, depends on our in-depth knowledge of their expression patterns and molecular mechanisms. 
Since, there are several TNF superfamily members that have the ability to influence neuroinflammation one way or another, reaching a decision about targeting one or more receptor-ligand pairs at a given time for clinical application requires further investigation. A better understanding of their expression profile, and kinetics of expression, and interactions between TNF ligands and their TNFRs on various CNS residents and infiltrating immune cells at different stages of the disease would help to design better strategies to control neuroinflammation and CNS autoimmunity.

\section{References}

1. Matsumoto M. Role of TNF ligand and receptor family in the lymphoid organogenesis defined by gene targeting. J Med Invest (1999) 46:141-50.

2. Ronchetti S, Nocentini G, Petrillo MG, Bianchini R, Sportoletti P, Bastianelli A, et al. Glucocorticoid-induced TNFR family related gene (GITR) enhances dendritic cell activity. Immunol Lett (2011) 135:24-33. doi:10.1016/j.imlet. 2010.09.008

3. Watts TH. TNF/TNFR family members in costimulation of $\mathrm{T}$ cell responses. Annu Rev Immunol (2005) 23:23-68. doi:10.1146/annurev.immunol.23. 021704.115839

4. Vincent FB, Morand EF, Schneider P, Mackay F. The BAFF/APRIL system in SLE pathogenesis. Nat Rev (2014) 10(6):365-73. doi:10.1038/nrrheum.2014.33

5. Croft M, Duan W, Choi H, Eun SY, Madireddi S, Mehta A. TNF superfamily in inflammatory disease: translating basic insights. Trends Immunol (2012) 33:144-52. doi:10.1016/j.it.2011.10.004

6. Croft M. The role of TNF superfamily members in T-cell function and diseases. Nat Rev Immunol (2009) 9:271-85. doi:10.1038/nri2526

7. Croft M, Benedict CA, Ware CF. Clinical targeting of the TNF and TNFR superfamilies. Nat Rev Drug Discov (2013) 12:147-68. doi:10.1038/ $\operatorname{nrd} 3930$

8. Dempsey PW, Doyle SE, He JQ, Cheng G. The signaling adaptors and pathways activated by TNF superfamily. Cytokine Growth Factor Rev (2003) 14:193-209. doi:10.1016/S1359-6101(03)00021-2

9. Wilson NS, Dixit V, Ashkenazi A. Death receptor signal transducers: nodes of coordination in immune signaling networks. Nat Immunol (2009) 10:348-55. doi:10.1038/ni.1714

10. Ashkenazi A, Dixit VM. Death receptors: signaling and modulation. Science (1998) 281:1305-8. doi:10.1126/science.281.5381.1305

11. Chung JY, Park YC, Ye H, Wu H. All TRAFs are not created equal: common and distinct molecular mechanisms of TRAF-mediated signal transduction. $J$ Cell Sci (2002) 115:679-88.

12. Kirchner S, Holler E, Haffner S, Andreesen R, Eissner G. Effect of different tumor necrosis factor (TNF) reactive agents on reverse signaling of membrane integrated TNF in monocytes. Cytokine (2004) 28:67-74. doi:10.1016/j.cyto. 2004.06.008

13. Ringheanu M, Daum F, Markowitz J, Levine J, Katz S, Lin X, et al. Effects of infliximab on apoptosis and reverse signaling of monocytes from healthy individuals and patients with Crohn's disease. Inflamm Bowel Dis (2004) 10:801-10. doi:10.1097/00054725-200411000-00015

14. Bennett J, Basivireddy J, Kollar A, Biron KE, Reickmann P, Jefferies WA, et al. Blood-brain barrier disruption and enhanced vascular permeability in the multiple sclerosis model EAE. J Neuroimmunol (2010) 229:180-91. doi:10. 1016/j.jneuroim.2010.08.011

15. Alvarez JI, Cayrol R, Prat A. Disruption of central nervous system barriers in multiple sclerosis. Biochim Biophys Acta (2011) 1812:252-64. doi:10.1016/j. bbadis.2010.06.017

16. Huppert J, Closhen D, Croxford A, White R, Kulig P, Pietrowski E, et al. Cellular mechanisms of IL-17-induced blood-brain barrier disruption. FASEB $J$ (2010) 24:1023-34. doi:10.1096/fj.09-141978

17. Tam SJ, Richmond DL, Kaminker JS, Modrusan Z, Martin-McNulty B, Cao TC, et al. Death receptors DR6 and TROY regulate brain vascular development. Dev Cell (2012) 22:403-17. doi:10.1016/j.devcel.2011.11.018

18. Daneman R, Agalliu D, Zhou L, Kuhnert F, Kuo CJ, Barres BA. Wnt/betacatenin signaling is required for CNS, but not non-CNS, angiogenesis. Proc Natl Acad Sci U S A (2009) 106:641-6. doi:10.1073/pnas.0805165106

\section{Acknowledgments}

We thank Dr. Jyoti Rao and Dr. Deepa Subramanyam for critical reading, helpful discussion, and editing the manuscript. This work was supported by the Department of Biotechnology, Government of India (BT/RLF/Re-entry/41/2010, BT/03/IYBA/2010, and BT/PR4610/MED/30/720/2012 to GL). SS is Senior Research Fellow (SRF) of Council of Scientific and Industrial Research, Government of India.

19. Liebner S, Corada M, Bangsow T, Babbage J, Taddei A, Czupalla CJ, et al. Wnt/beta-catenin signaling controls development of the blood-brain barrier. J Cell Biol (2008) 183:409-17. doi:10.1083/jcb.200806024

20. Hofman FM, Hinton DR, Johnson K, Merrill JE. Tumor necrosis factor identified in multiple sclerosis brain. J Exp Med (1989) 170:607-12. doi:10.1084/ jem.170.2.607

21. Kaltsonoudis E, Voulgari PV, Konitsiotis S, Drosos AA. Demyelination and other neurological adverse events after anti-TNF therapy. Autoimmun Rev (2014) 13:54-8. doi:10.1016/j.autrev.2013.09.002

22. Korner H, Lemckert FA, Chaudhri G, Etteldorf S, Sedgwick JD. Tumor necrosis factor blockade in actively induced experimental autoimmune encephalomyelitis prevents clinical disease despite activated $\mathrm{T}$ cell infiltration to the central nervous system. Eur J Immunol (1997) 27:1973-81. doi:10.1002/ eji. 1830270822

23. Sharief MK, Hentges R. Association between tumor necrosis factor-alpha and disease progression in patients with multiple sclerosis. N Engl J Med (1991) 325:467-72. doi:10.1056/NEJM199108153250704

24. Sharief MK, Hentges R, Ciardi M. Intrathecal immune response in patients with the post-polio syndrome. N Engl J Med (1991) 325:749-55. doi:10.1056/ NEJM199109123251101

25. Korner H, Riminton DS, Strickland DH, Lemckert FA, Pollard JD, Sedgwick JD. Critical points of tumor necrosis factor action in central nervous system autoimmune inflammation defined by gene targeting. J Exp Med (1997) 186:1585-90. doi:10.1084/jem.186.9.1585

26. Liu J, Marino MW, Wong G, Grail D, Dunn A, Bettadapura J, et al. TNF is a potent anti-inflammatory cytokine in autoimmune-mediated demyelination. Nat Med (1998) 4:78-83. doi:10.1038/nm0198-078

27. Probert L, Akassoglou K, Pasparakis M, Kontogeorgos G, Kollias G. Spontaneous inflammatory demyelinating disease in transgenic mice showing central nervous system-specific expression of tumor necrosis factor alpha. Proc Natl Acad Sci U S A (1995) 92:11294-8. doi:10.1073/pnas.92.24.11294

28. Ruddle NH, Bergman CM, McGrath KM, Lingenheld EG, Grunnet ML, Padula SJ, et al. An antibody to lymphotoxin and tumor necrosis factor prevents transfer of experimental allergic encephalomyelitis. J Exp Med (1990) 172:1193-200. doi:10.1084/jem.172.4.1193

29. Eugster HP, Frei K, Winkler F, Koedel U, Pfister W, Lassmann H, et al. Superantigen overcomes resistance of IL-6-deficient mice towards MOG-induced EAE by a TNFR1 controlled pathway. Eur J Immunol (2001) 31:2302-12. doi:10.1002/1521-4141(200108)31:8<2302::AID-IMMU2302>3.0.CO;2-\#

30. Eugster HP, Frei K, Bachmann R, Bluethmann H, Lassmann H, Fontana A. Severity of symptoms and demyelination in MOG-induced EAE depends on TNFR1. Eur J Immunol (1999) 29:626-32. doi:10.1002/(SICI) 1521-4141(199902)29:02<626::AID-IMMU626>3.0.CO;2-A

31. TNF neutralization in MS: results of a randomized, placebo-controlled multicenter study. The Lenercept Multiple Sclerosis Study Group and The University of British Columbia MS/MRI Analysis Group. Neurology (1999) 53:457-65. doi:10.1212/WNL.53.3.457

32. Kaltsonoudis E, Zikou AK, Voulgari PV, Konitsiotis S, Argyropoulou MI, Drosos AA. Neurological adverse events in patients receiving anti-TNF therapy: a prospective imaging and electrophysiological study. Arthritis Res Ther (2014) 16:R125. doi:10.1186/ar4582

33. van Boxel-Dezaire AH, Hoff SC, van Oosten BW, Verweij CL, Drager AM, Ader HJ, et al. Decreased interleukin-10 and increased interleukin-12p40 mRNA are associated with disease activity and characterize different disease stages in multiple sclerosis. Ann Neurol (1999) 45:695-703. doi:10.1002/ 1531-8249(199906)45:6<695::AID-ANA3>3.0.CO;2-R 
34. Magnano MD, Robinson WH, Genovese MC. Demyelination and inhibition of tumor necrosis factor (TNF). Clin Exp Rheumatol (2004) 22:S134-40.

35. Robinson WH, Genovese MC, Moreland LW. Demyelinating and neurologic events reported in association with tumor necrosis factor alpha antagonism: by what mechanisms could tumor necrosis factor alpha antagonists improve rheumatoid arthritis but exacerbate multiple sclerosis? Arthritis Rheum (2001) 44:1977-83. doi:10.1002/1529-0131(200109)44:9<1977:: AID-ART345>3.0.CO;2-6

36. Prinz JC. Autoimmune-like syndromes during TNF blockade: does infection have a role? Nat Rev (2011) 7:429-34. doi:10.1038/nrrheum.2011.35

37. Watanabe-Fukunaga R, Brannan CI, Copeland NG, Jenkins NA, Nagata S, Lymphoproliferation disorder in mice explained by defects in Fas antigen that mediates apoptosis. Nature (1992) 356:314-7. doi:10.1038/356314a0

38. Takahashi T, Tanaka M, Brannan CI, Jenkins NA, Copeland NG, Suda T, et al. Generalized lymphoproliferative disease in mice, caused by a point mutation in the Fas ligand. Cell (1994) 76:969-76. doi:10.1016/0092-8674(94)90375-1

39. Waldner H, Sobel RA, Howard E, Kuchroo VK. Fas- and FasL-deficient mice are resistant to induction of autoimmune encephalomyelitis. J Immunol (1997) 159:3100-3.

40. Suvannavejh GC, Dal Canto MC, Matis LA, Miller SD. Fas-mediated apoptosis in clinical remissions of relapsing experimental autoimmune encephalomyelitis. J Clin Invest (2000) 105:223-31. doi:10.1172/JCI8561

41. Okuda Y, Sakoda S, Fujimura H, Nagata S, Yanagihara T, Bernard CC. Intrathecal administration of neutralizing antibody against Fas ligand suppresses the progression of experimental autoimmune encephalomyelitis. Biochem Biophys Res Commun (2000) 275:164-8. doi:10.1006/bbrc.2000.3279

42. Zhu B, Luo L, Chen Y, Paty DW, Cynader MS. Intrathecal Fas ligand infusion strengthens immunoprivilege of central nervous system and suppresses experimental autoimmune encephalomyelitis. J Immunol (2002) 169:1561-9. doi:10.4049/jimmunol.169.3.1561

43. Sabelko-Downes KA, Cross AH, Russell JH. Dual role for Fas ligand in the initiation of and recovery from experimental allergic encephalomyelitis. J Exp Med (1999) 189:1195-205. doi:10.1084/jem.189.8.1195

44. Voskuhl RR, Peterson RS, Song B, Ao Y, Morales LB, Tiwari-Woodruff S, et al. Reactive astrocytes form scar-like perivascular barriers to leukocytes during adaptive immune inflammation of the CNS. J Neurosci (2009) 29:11511-22. doi:10.1523/JNEUROSCI.1514-09.2009

45. Wang X, Haroon F, Karray S, Martina D, Schluter D. Astrocytic Fas ligand expression is required to induce T-cell apoptosis and recovery from experimental autoimmune encephalomyelitis. Eur J Immunol (2013) 43:115-24. doi:10.1002/eji.201242679

46. Paul S, Singh AK, Shilpi, Lal G. Phenotypic and functional plasticity of gammadelta (yd) T cells in inflammation and tolerance. Int Rev Immunol (2014) 33:537-58. doi:10.3109/08830185.2013.863306

47. Ponomarev ED, Dittel BN. Gamma delta T cells regulate the extent and duration of inflammation in the central nervous system by a Fas ligand-dependent mechanism. J Immunol (2005) 174:4678-87. doi:10.4049/jimmunol.174.8. 4678

48. Schneider P, MacKay F, Steiner V, Hofmann K, Bodmer JL, Holler N, et al. BAFF, a novel ligand of the tumor necrosis factor family, stimulates B cell growth. J Exp Med (1999) 189:1747-56. doi:10.1084/jem.189.11.1747

49. Chu VT, Enghard P, Riemekasten G, Berek C. In vitro and in vivo activation induces BAFF and APRIL expression in B cells. J Immunol (2007) 179:5947-57. doi:10.4049/jimmunol.179.9.5947

50. Mackay F, Woodcock SA, Lawton P, Ambrose C, Baetscher M, Schneider P, et al. Mice transgenic for BAFF develop lymphocytic disorders along with autoimmune manifestations. J Exp Med (1999) 190:1697-710. doi:10.1084/ jem.190.11.1697

51. Stadanlick JE, Kaileh M, Karnell FG, Scholz JL, Miller JP, Quinn WJ III, et al. Tonic B cell antigen receptor signals supply an NF-kappaB substrate for prosurvival BLyS signaling. Nat Immunol (2008) 9:1379-87. doi:10.1038/ni. 1666

52. Meyer-Bahlburg A, Andrews SF, Yu KO, Porcelli SA, Rawlings DJ. Characterization of a late transitional B cell population highly sensitive to BAFFmediated homeostatic proliferation. J Exp Med (2008) 205:155-68. doi:10. 1084/jem. 20071088

53. Gross JA, Johnston J, Mudri S, Enselman R, Dillon SR, Madden K, et al. TACI and BCMA are receptors for a TNF homologue implicated in B-cell autoimmune disease. Nature (2000) 404:995-9. doi:10.1038/35010115
54. Rickert RC, Jellusova J, Miletic AV. Signaling by the tumor necrosis factor receptor superfamily in B-cell biology and disease. Immunol Rev (2011) 244:115-33. doi:10.1111/j.1600-065X.2011.01067.x

55. Schneider P, Takatsuka H, Wilson A, Mackay F, Tardivel A, Lens S, et al. Maturation of marginal zone and follicular B cells requires B cell activating factor of the tumor necrosis factor family and is independent of B cell maturation antigen. J Exp Med (2001) 194:1691-7. doi:10.1084/jem.194.11.1691

56. Marsters SA, Yan M, Pitti RM, Haas PE, Dixit VM, Ashkenazi A. Interaction of the TNF homologues BLyS and APRIL with the TNF receptor homologues BCMA and TACI. Curr Biol (2000) 10:785-8. doi:10.1016/S0960-9822(00) 00566-2

57. Kim SS, Richman DP, Zamvil SS, Agius MA. Accelerated central nervous system autoimmunity in BAFF-receptor-deficient mice. J Neurol Sci (2011) 306:9-15. doi:10.1016/j.jns.2011.04.008

58. Lee-Chang C, Lefranc D, Salleron J, Faveeuw C, Allet C, Vermersch P, et al. Susceptibility to experimental autoimmune encephalomyelitis is associated with altered B-cell subsets distribution and decreased serum BAFF levels. Immunol Lett (2011) 135:108-17. doi:10.1016/j.imlet.2010.10.006

59. Huntington ND, Tomioka R, Clavarino C, Chow AM, Linares D, Mana P, et al. A BAFF antagonist suppresses experimental autoimmune encephalomyelitis by targeting cell-mediated and humoral immune responses. Int Immunol (2006) 18:1473-85. doi:10.1093/intimm/dxl080

60. Zhou X, Xia Z, Lan Q, Wang J, Su W, Han YP, et al. BAFF promotes Th17 cells and aggravates experimental autoimmune encephalomyelitis. PLoS One (2011) 6:e23629. doi:10.1371/journal.pone.0023629

61. Jagessar SA, Heijmans N, Oh L, Bauer J, Blezer EL, Laman JD, et al. Antibodies against human BLyS and APRIL attenuate EAE development in marmoset monkeys. J Neuroimmune Pharmacol (2012) 7:557-70. doi:10.1007/ s11481-012-9384-x

62. Kala M, Miravalle A, Vollmer T. Recent insights into the mechanism of action of glatiramer acetate. J Neuroimmunol (2011) 235:9-17. doi:10.1016/ j.jneuroim.2011.01.009

63. Aharoni R, Vainshtein A, Stock A, Eilam R, From R, Shinder V, et al. Distinct pathological patterns in relapsing-remitting and chronic models of experimental autoimmune encephalomyelitis and the neuroprotective effect of glatiramer acetate. J Autoimmun (2011) 37:228-41. doi:10.1016/j.jaut.2011.06.003

64. Arnon R, Aharoni R. Neuroprotection and neurogeneration in MS and its animal model EAE effected by glatiramer acetate. J Neural Transm (2009) 116:1443-9. doi:10.1007/s00702-009-0272-3

65. Kala M, Rhodes SN, Piao WH, Shi FD, Campagnolo DI, Vollmer TL. B cells from glatiramer acetate-treated mice suppress experimental autoimmune encephalomyelitis. Exp Neurol (2010) 221:136-45. doi:10.1016/j.expneurol. 2009.10.015

66. Begum-Haque S, Sharma A, Christy M, Lentini T, Ochoa-Reparaz J, Fayed IF, et al. Increased expression of B cell-associated regulatory cytokines by glatiramer acetate in mice with experimental autoimmune encephalomyelitis. J Neuroimmunol (2010) 219:47-53. doi:10.1016/j.jneuroim.2009.11.016

67. Valzasina B, Guiducci C, Dislich H, Killeen N, Weinberg AD, Colombo MP. Triggering of OX40 (CD134) on CD4(+)CD25+ T cells blocks their inhibitory activity: a novel regulatory role for OX40 and its comparison with GITR. Blood (2005) 105:2845-51. doi:10.1182/blood-2004-07-2959

68. Croft M. Control of immunity by the TNFR-related molecule OX40 (CD134). Annu Rev Immunol (2010) 28:57-78. doi:10.1146/annurev-immunol-030409101243

69. Chen AI, McAdam AJ, Buhlmann JE, Scott S, Lupher ML Jr, Greenfield EA, et al. Ox40-ligand has a critical costimulatory role in dendritic cell:T cell interactions. Immunity (1999) 11:689-98. doi:10.1016/S1074-7613(00) 80143-0

70. Weinberg AD. OX40: targeted immunotherapy - implications for tempering autoimmunity and enhancing vaccines. Trends Immunol (2002) 23:102-9. doi:10.1016/S1471-4906(01)02127-5

71. Chitnis T, Najafian N, Abdallah KA, Dong V, Yagita H, Sayegh MH, et al. CD28-independent induction of experimental autoimmune encephalomyelitis. J Clin Invest (2001) 107:575-83. doi:10.1172/JCI11220

72. Ruby CE, Montler R, Zheng R, Shu S, Weinberg AD. IL-12 is required for antiOX40-mediated CD4 T cell survival. J Immunol (2008) 180:2140-8. doi:10. 4049/jimmunol.180.4.2140

73. Prell RA, Evans DE, Thalhofer C, Shi T, Funatake C, Weinberg AD. OX40mediated memory $\mathrm{T}$ cell generation is TNF receptor-associated factor 2 
dependent. J Immunol (2003) 171:5997-6005. doi:10.4049/jimmunol.171.11. 5997

74. So T, Croft M. Cutting edge: OX40 inhibits TGF-beta- and antigen-driven conversion of naive CD4 T cells into CD25+Foxp3+ T cells. J Immunol (2007) 179:1427-30. doi:10.4049/jimmunol.179.3.1427

75. Vu MD, Xiao X, Gao W, Degauque N, Chen M, Kroemer A, et al. OX40 costimulation turns off Foxp3+ Tregs. Blood (2007) 110:2501-10. doi:10.1182/ blood-2007-01-070748

76. Chen M, Xiao X, Demirci G, Li XC. OX40 controls islet allograft tolerance in CD154 deficient mice by regulating FOXP3+ Tregs. Transplantation (2008) 85:1659-62. doi:10.1097/TP.0b013e3181726987

77. Ito T, Wang YH, Duramad O, Hanabuchi S, Perng OA, Gilliet M, et al. OX40 ligand shuts down IL-10-producing regulatory T cells. Proc Natl Acad Sci U S A (2006) 103:13138-43. doi:10.1073/pnas.0603107103

78. Mahmud SA, Manlove LS, Schmitz HM, Xing Y, Wang Y, Owen DL, et al. Costimulation via the tumor-necrosis factor receptor superfamily couples TCR signal strength to the thymic differentiation of regulatory T cells. Nat Immunol (2014) 15:473-81. doi:10.1038/ni.2849

79. Xiao X, Kroemer A, Gao W, Ishii N, Demirci G, Li XC. OX40/OX40L costimulation affects induction of Foxp3+ regulatory $\mathrm{T}$ cells in part by expanding memory $\mathrm{T}$ cells in vivo. J Immunol (2008) 181:3193-201. doi:10.4049/ jimmunol.181.5.3193

80. Xiao X, Balasubramanian S, Liu W, Chu X, Wang H, Taparowsky EJ, et al. OX40 signaling favors the induction of $\mathrm{T}(\mathrm{H}) 9$ cells and airway inflammation. Nat Immunol (2012) 13:981-90. doi:10.1038/ni.2390

81. Weinberg AD, Bourdette DN, Sullivan TJ, Lemon M, Wallin JJ, Maziarz R, et al. Selective depletion of myelin-reactive T cells with the anti-OX-40 antibody ameliorates autoimmune encephalomyelitis. Nat Med (1996) 2:183-9. doi:10. 1038/nm0296-183

82. Weinberg AD, Wegmann KW, Funatake C, Whitham RH. Blocking OX40/OX-40 ligand interaction in vitro and in vivo leads to decreased $\mathrm{T}$ cell function and amelioration of experimental allergic encephalomyelitis. J Immunol (1999) 162:1818-26.

83. Kotani A, Hori T, Matsumura Y, Uchiyama T. Signaling of gp34 (OX40 ligand) induces vascular endothelial cells to produce a CC chemokine RANTES/CCL5. Immunol Lett (2002) 84:1-7. doi:10.1016/S0165-2478(02) 00082-2

84. Nohara C, Akiba H, Nakajima A, Inoue A, Koh CS, Ohshima H, et al. Amelioration of experimental autoimmune encephalomyelitis with anti-OX40 ligand monoclonal antibody: a critical role for OX40 ligand in migration, but not development, of pathogenic T cells. J Immunol (2001) 166:2108-15. doi:10.4049/jimmunol.166.3.2108

85. Yeo YA, Martinez Gomez JM, Croxford JL, Gasser S, Ling EA, Schwarz H. CD137 ligand activated microglia induces oligodendrocyte apoptosis via reactive oxygen species. J Neuroinflammation (2012) 9:173. doi:10.1186/ 1742-2094-9-173

86. Saoulli K, Lee SY, Cannons JL, Yeh WC, Santana A, Goldstein MD, et al. CD28independent, TRAF2-dependent costimulation of resting $\mathrm{T}$ cells by $4-1 \mathrm{BB}$ ligand. J Exp Med (1998) 187:1849-62. doi:10.1084/jem.187.11.1849

87. Sun Y, Lin X, Chen HM, Wu Q, Subudhi SK, Chen L, et al. Administration of agonistic anti-4-1BB monoclonal antibody leads to the amelioration of experimental autoimmune encephalomyelitis. J Immunol (2002) 168:1457-65. doi:10.4049/jimmunol.168.3.1457

88. Mittler RS, Bailey TS, Klussman K, Trailsmith MD, Hoffmann MK. Anti4-1BB monoclonal antibodies abrogate $\mathrm{T}$ cell-dependent humoral immune responses in vivo through the induction of helper T cell anergy. J Exp Med (1999) 190:1535-40. doi:10.1084/jem.190.10.1535

89. Martinez Gomez JM, Croxford JL, Yeo KP, Angeli V, Schwarz H, Gasser S. Development of experimental autoimmune encephalomyelitis critically depends on CD137 ligand signaling. J Neurosci (2012) 32:18246-52. doi:10. 1523/JNEUROSCI.2473-12.2012

90. Carrier Y, Whitters MJ, Miyashiro JS, LaBranche TP, Ramon HE, Benoit SE, et al. Enhanced GITR/GITRL interactions augment IL-27 expression and induce IL-10-producing Tr-1 like cells. Eur J Immunol (2012) 42:1393-404. doi:10.1002/eji.201142162

91. Coe D, Begom S, Addey C, White M, Dyson J, Chai JG. Depletion of regulatory $\mathrm{T}$ cells by anti-GITR $\mathrm{mAb}$ as a novel mechanism for cancer immunotherapy. Cancer Immunol Immunother (2010) 59:1367-77. doi:10. 1007/s00262-010-0866-5
92. Shimizu J, Yamazaki S, Takahashi T, Ishida Y, Sakaguchi S. Stimulation of $\mathrm{CD} 25(+) \mathrm{CD} 4(+)$ regulatory $\mathrm{T}$ cells through GITR breaks immunological selftolerance. Nat Immunol (2002) 3:135-42. doi:10.1038/ni759

93. Kanamaru F, Youngnak P, Hashiguchi M, Nishioka T, Takahashi T, Sakaguchi $\mathrm{S}$, et al. Costimulation via glucocorticoid-induced TNF receptor in both conventional and CD25+ regulatory CD4+ T cells. J Immunol (2004) 172:7306-14. doi:10.4049/jimmunol.172.12.7306

94. Ronchetti S, Nocentini G, Bianchini R, Krausz LT, Migliorati G, Riccardi C. Glucocorticoid-induced TNFR-related protein lowers the threshold of CD28 costimulation in CD8+ T cells. J Immunol (2007) 179:5916-26. doi:10.4049/ jimmunol.179.9.5916

95. Ronchetti S, Zollo O, Bruscoli S, Agostini M, Bianchini R, Nocentini G, et al. GITR, a member of the TNF receptor superfamily, is costimulatory to mouse T lymphocyte subpopulations. Eur J Immunol (2004) 34:613-22. doi:10.1002/ eji.200324804

96. Lal G, Nakayama Y, Sethi A, Singh AK, Burrell BE, Kulkarni N, et al. Interleukin-10 from marginal zone precursor B-cell subset is required for costimulatory blockade-induced transplantation tolerance. Transplantation (2015). doi:10.1097/TP.0000000000000718

97. Ray A, Mann MK, Basu S, Dittel BN. A case for regulatory B cells in controlling the severity of autoimmune-mediated inflammation in experimental autoimmune encephalomyelitis and multiple sclerosis. J Neuroimmunol (2011) 230:1-9. doi:10.1016/j.jneuroim.2010.10.037

98. Goetz M, Atreya R, Ghalibafian M, Galle PR, Neurath MF. Exacerbation of ulcerative colitis after rituximab salvage therapy. Inflamm Bowel Dis (2007) 13:1365-8. doi:10.1002/ibd.20215

99. van Olffen RW, Koning N, van Gisbergen KP, Wensveen FM, Hoek RM, Boon $\mathrm{L}$, et al. GITR triggering induces expansion of both effector and regulatory CD4+ T cells in vivo. J Immunol (2009) 182:7490-500. doi:10.4049/jimmunol. 0802751

100. Ray A, Basu S, Williams CB, Salzman NH, Dittel BN. A novel IL-10independent regulatory role for $\mathrm{B}$ cells in suppressing autoimmunity by maintenance of regulatory T cells via GITR ligand. J Immunol (2012) 188:3188-98. doi:10.4049/jimmunol.1103354

101. Hoffmann O, Zipp F, Weber JR. Tumour necrosis factor-related apoptosisinducing ligand (TRAIL) in central nervous system inflammation. J Mol Med (Berl) (2009) 87:753-63. doi:10.1007/s00109-009-0484-x

102. Cretney E, McQualter JL, Kayagaki N, Yagita H, Bernard CC, Grewal IS, et al. TNF-related apoptosis-inducing ligand (TRAIL)/Apo2L suppresses experimental autoimmune encephalomyelitis in mice. Immunol Cell Biol (2005) 83:511-9. doi:10.1111/j.1440-1711.2005.01358.x

103. Ikeda T, Hirata S, Fukushima S, Matsunaga Y, Ito T, Uchino M, et al. Dual effects of TRAIL in suppression of autoimmunity: the inhibition of Th1 cells and the promotion of regulatory T cells. J Immunol (2010) 185:5259-67. doi:10.4049/jimmunol.0902797

104. Hirata S, Matsuyoshi H, Fukuma D, Kurisaki A, Uemura Y, Nishimura Y, et al. Involvement of regulatory $\mathrm{T}$ cells in the experimental autoimmune encephalomyelitis-preventive effect of dendritic cells expressing myelin oligodendrocyte glycoprotein plus TRAIL. J Immunol (2007) 178:918-25. doi:10. 4049/jimmunol.178.2.918

105. Hirata S, Senju S, Matsuyoshi H, Fukuma D, Uemura Y, Nishimura Y. Prevention of experimental autoimmune encephalomyelitis by transfer of embryonic stem cell-derived dendritic cells expressing myelin oligodendrocyte glycoprotein peptide along with TRAIL or programmed death-1 ligand. J Immunol (2005) 174:1888-97. doi:10.4049/jimmunol.174.4.1888

106. Emery JG, McDonnell P, Burke MB, Deen KC, Lyn S, Silverman C, et al. Osteoprotegerin is a receptor for the cytotoxic ligand TRAIL. J Biol Chem (1998) 273:14363-7. doi:10.1074/jbc.273.23.14363

107. Cheng E, Armstrong CL, Galisteo R, Winkles JA. TWEAK/Fn14 axis-targeted therapeutics: moving basic science discoveries to the clinic. Front Immunol (2013) 4:473. doi:10.3389/fimmu.2013.00473

108. Potrovita I, Zhang W, Burkly L, Hahm K, Lincecum J, Wang MZ, et al. Tumor necrosis factor-like weak inducer of apoptosis-induced neurodegeneration. J Neurosci (2004) 24:8237-44. doi:10.1523/JNEUROSCI.1089-04. 2004

109. Desplat-Jego S, Varriale S, Creidy R, Terra R, Bernard D, Khrestchatisky $\mathrm{M}$, et al. TWEAK is expressed by glial cells, induces astrocyte proliferation and increases EAE severity. J Neuroimmunol (2002) 133:116-23. doi:10.1016/ S0165-5728(02)00368-5 
110. Mueller AM, Pedre X, Kleiter I, Hornberg M, Steinbrecher A, Giegerich G. Targeting fibroblast growth factor-inducible-14 signaling protects from chronic relapsing experimental autoimmune encephalomyelitis. J Neuroimmunol (2005) 159:55-65. doi:10.1016/j.jneuroim.2004.10.001

111. Sagar D, Lamontagne A, Foss CA, Khan ZK, Pomper MG, Jain P. Dendritic cell CNS recruitment correlates with disease severity in EAE via CCL2 chemotaxis at the blood-brain barrier through paracellular transmigration and ERK activation. J Neuroinflammation (2012) 9:245. doi:10.1186/1742-20949-245

112. Moreno M, Bannerman P, Ma J, Guo F, Miers L, Soulika AM, et al. Conditional ablation of astroglial CCL2 suppresses CNS accumulation of M1 macrophages and preserves axons in mice with MOG peptide EAE. J Neurosci (2014) 34:8175-85. doi:10.1523/JNEUROSCI.1137-14.2014

113. Zhang X, Winkles JA, Gongora MC, Polavarapu R, Michaelson JS, Hahm K, et al. TWEAK-Fn14 pathway inhibition protects the integrity of the neurovascular unit during cerebral ischemia. J Cereb Blood Flow Metab (2007) 27:534-44. doi:10.1038/sj.jcbfm.9600368

114. Polavarapu R, Gongora MC, Winkles JA, Yepes M. Tumor necrosis factorlike weak inducer of apoptosis increases the permeability of the neurovascular unit through nuclear factor-kappa B pathway activation. J Neurosci (2005) 25:10094-100. doi:10.1523/JNEUROSCI.3382-05.2005

115. Roos C, Wicovsky A, Muller N, Salzmann S, Rosenthal T, Kalthoff H, et al. Soluble and transmembrane TNF-like weak inducer of apoptosis differentially activate the classical and noncanonical NF-kappa B pathway. J Immunol (2010) 185:1593-605. doi:10.4049/jimmunol.0903555

116. Desplat-Jego S, Creidy R, Varriale S, Allaire N, Luo Y, Bernard D, et al. Anti-TWEAK monoclonal antibodies reduce immune cell infiltration in the central nervous system and severity of experimental autoimmune encephalomyelitis. Clin Immunol (2005) 117:15-23. doi:10.1016/j.clim.2005. 06.005

117. Perper SJ, Browning B, Burkly LC, Weng S, Gao C, Giza K, et al. TWEAK is a novel arthritogenic mediator. J Immunol (2006) 177:2610-20. doi:10.4049/ jimmunol.177.4.2610

118. Razmara M, Hilliard B, Ziarani AK, Murali R, Yellayi S, Ghazanfar M, et al. Fn14-TRAIL, a chimeric intercellular signal exchanger, attenuates experimental autoimmune encephalomyelitis. Am J Pathol (2009) 174:460-74. doi:10. 2353/ajpath.2009.080462

119. Prinz-Hadad H, Mizrachi T, Irony-Tur-Sinai M, Prigozhina TB, Aronin A, Brenner T, et al. Amelioration of autoimmune neuroinflammation by the fusion molecule Fn14.TRAIL. J Neuroinflammation (2013) 10:36. doi:10.1186/ 1742-2094-10-36

120. Nolte MA, van Olffen RW, van Gisbergen KP, van Lier RA. Timing and tuning of CD27-CD70 interactions: the impact of signal strength in setting the balance between adaptive responses and immunopathology. Immunol Rev (2009) 229:216-31. doi:10.1111/j.1600-065X.2009.00774.x

121. Nakajima A, Oshima H, Nohara C, Morimoto S, Yoshino S, Kobata T, et al. Involvement of CD70-CD27 interactions in the induction of experimental autoimmune encephalomyelitis. J Neuroimmunol (2000) 109:188-96. doi:10. 1016/S0165-5728(00)00324-6

122. Ribot JC, deBarros A, Pang DJ, Neves JF, Peperzak V, Roberts SJ, et al. CD27 is a thymic determinant of the balance between interferon-gammaand interleukin 17-producing gammadelta T cell subsets. Nat Immunol (2009) 10:427-36. doi:10.1038/ni.1717

123. Paul S, Shilpi, Lal G. Role of gamma-delta (gammadelta) T cells in autoimmunity. J Leukoc Biol (2015) 97:259-71. doi:10.1189/jlb.3RU0914-443R

124. Coquet JM, Middendorp S, van der Horst G, Kind J, Veraar EA, Xiao Y, et al. The CD27 and CD70 costimulatory pathway inhibits effector function of T helper 17 cells and attenuates associated autoimmunity. Immunity (2013) 38:53-65. doi:10.1016/j.immuni.2012.09.009

125. Wang X, Dong C. The CD70-CD27 axis, a new brake in the T helper 17 cell response. Immunity (2013) 38:1-3. doi:10.1016/j.immuni.2013.01.005

126. Francosalinas G, Cantaert T, Nolte MA, Tak PP, van Lier RA, Baeten DL. Enhanced costimulation by $\mathrm{CD} 70+\mathrm{B}$ cells aggravates experimental autoimmune encephalomyelitis in autoimmune mice. J Neuroimmunol (2013) 255:8-17. doi:10.1016/j.jneuroim.2012.10.010
127. Tesselaar K, Arens R, van Schijndel GM, Baars PA, van der Valk MA, Borst $\mathrm{J}$, et al. Lethal $\mathrm{T}$ cell immunodeficiency induced by chronic costimulation via CD27-CD70 interactions. Nat Immunol (2003) 4:49-54. doi:10.1038/ni869

128. Gommerman JL, Giza K, Perper S, Sizing I, Ngam-Ek A, Nickerson-Nutter $\mathrm{C}$, et al. A role for surface lymphotoxin in experimental autoimmune encephalomyelitis independent of LIGHT. J Clin Invest (2003) 112:755-67. doi:10.1172/JCI18648

129. De Togni P, Goellner J, Ruddle NH, Streeter PR, Fick A, Mariathasan S, et al. Abnormal development of peripheral lymphoid organs in mice deficient in lymphotoxin. Science (1994) 264:703-7. doi:10.1126/science.8171322

130. Suen WE, Bergman CM, Hjelmstrom P, Ruddle NH. A critical role for lymphotoxin in experimental allergic encephalomyelitis. J Exp Med (1997) 186:1233-40. doi:10.1084/jem.186.8.1233

131. Mana P, Linares D, Silva DG, Fordham S, Scheu S, Pfeffer K, et al. LIGHT (TNFSF14/CD258) is a decisive factor for recovery from experimental autoimmune encephalomyelitis. J Immunol (2013) 191:154-63. doi:10.4049/ jimmunol.1203016

132. Gerritse K, Laman JD, Noelle RJ, Aruffo A, Ledbetter JA, Boersma WJ, et al. CD40-CD40 ligand interactions in experimental allergic encephalomyelitis and multiple sclerosis. Proc Natl Acad Sci U S A (1996) 93:2499-504. doi:10. 1073/pnas.93.6.2499

133. Ponomarev ED, Shriver LP, Dittel BN. CD40 expression by microglial cells is required for their completion of a two-step activation process during central nervous system autoimmune inflammation. J Immunol (2006) 176:1402-10. doi:10.4049/jimmunol.176.3.1402

134. Kim DY, Hong GU, Ro JY. Signal pathways in astrocytes activated by cross-talk between of astrocytes and mast cells through CD40-CD40L. JNeuroinflammation (2011) 8:25. doi:10.1186/1742-2094-8-25

135. Girvin AM, Dal Canto MC, Miller SD. CD40/CD40L interaction is essential for the induction of EAE in the absence of CD28-mediated co-stimulation. $J$ Autoimmun (2002) 18:83-94. doi:10.1006/jaut.2001.0573

136. Grewal IS, Foellmer HG, Grewal KD, Xu J, Hardardottir F, Baron JL, et al. Requirement for CD40 ligand in costimulation induction, $\mathrm{T}$ cell activation, and experimental allergic encephalomyelitis. Science (1996) 273:1864-7. doi:10.1126/science.273.5283.1864

137. Howard LM, Dal Canto MC, Miller SD. Transient anti-CD154-mediated immunotherapy of ongoing relapsing experimental autoimmune encephalomyelitis induces long-term inhibition of disease relapses. $J$ Neuroimmunol (2002) 129:58-65. doi:10.1016/S0165-5728(02)00175-3

138. Ndhlovu LC, Ishii N, Murata K, Sato T, Sugamura K. Critical involvement of OX40 ligand signals in the $\mathrm{T}$ cell priming events during experimental autoimmune encephalomyelitis. J Immunol (2001) 167:2991-9. doi:10.4049/ jimmunol.167.5.2991

139. Becher B, Durell BG, Miga AV, Hickey WF, Noelle RJ. The clinical course of experimental autoimmune encephalomyelitis and inflammation is controlled by the expression of CD40 within the central nervous system. J Exp Med (2001) 193:967-74. doi:10.1084/jem.193.8.967

140. Iezzi G, Sonderegger I, Ampenberger F, Schmitz N, Marsland BJ, Kopf M. CD40-CD40L cross-talk integrates strong antigenic signals and microbial stimuli to induce development of IL-17-producing CD4+ T cells. Proc Natl Acad Sci U S A (2009) 106:876-81. doi:10.1073/pnas.0810769106

141. Wang Y, Subudhi SK, Anders RA, Lo J, Sun Y, Blink S, et al. The role of herpesvirus entry mediator as a negative regulator of $\mathrm{T}$ cell-mediated responses. J Clin Invest (2005) 115:711-7. doi:10.1172/JCI22982

Conflict of Interest Statement: The authors declare that the research was conducted in the absence of any commercial or financial relationships that could be construed as a potential conflict of interest.

Copyright (C) 2015 Sonar and Lal. This is an open-access article distributed under the terms of the Creative Commons Attribution License (CC BY). The use, distribution or reproduction in other forums is permitted, provided the original author(s) or licensor are credited and that the original publication in this journal is cited, in accordance with accepted academic practice. No use, distribution or reproduction is permitted which does not comply with these terms. 


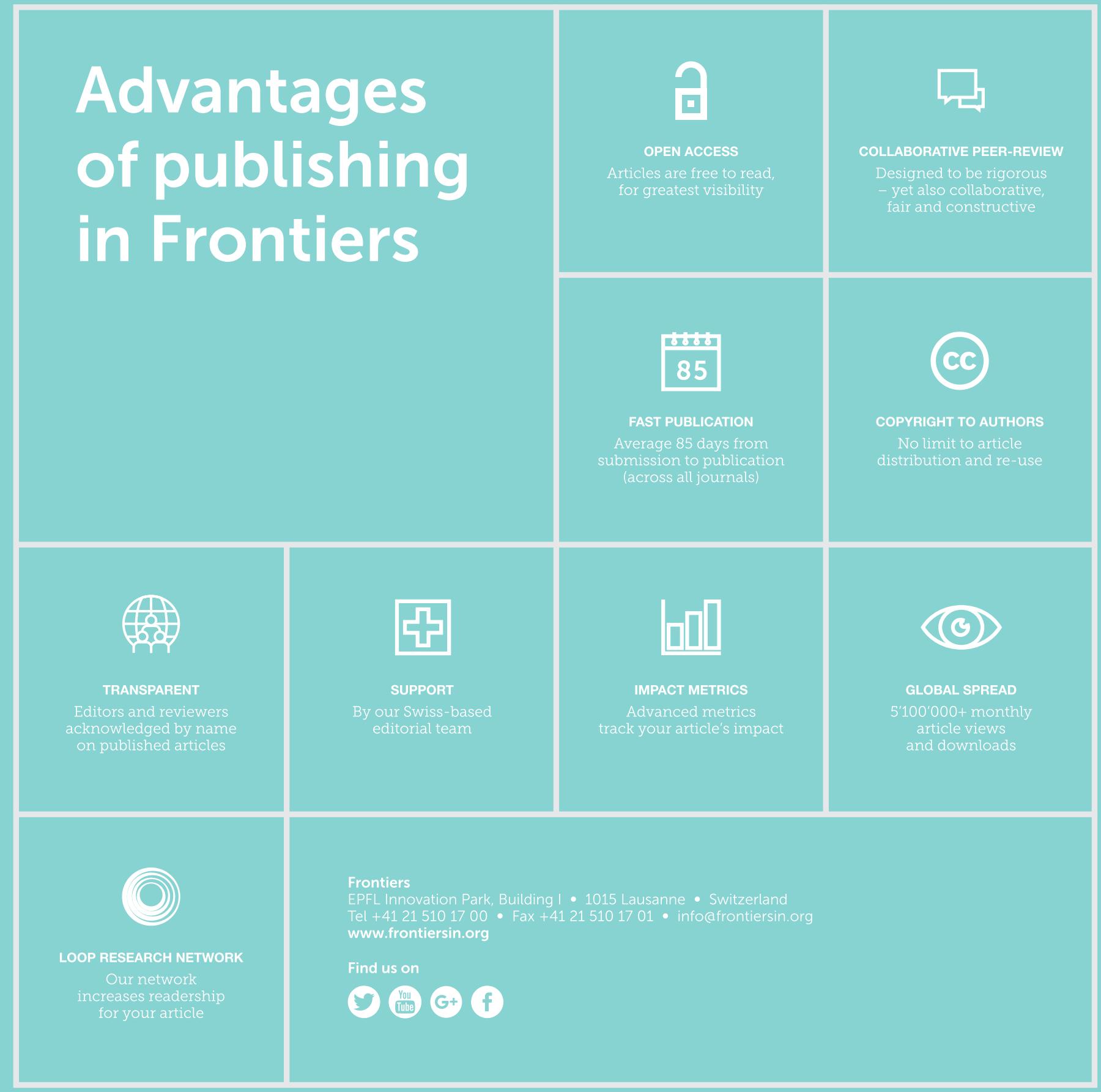

